\title{
BIO-FUNCTIONALIZATION OF SILICON AND ITS APPLICATIONS IN MAMMALIAN AND CANCER CELL MANIPULATION AND PROLIFERATION
}

\author{
by \\ Priyatha Premnath \\ Master of Engineering, Cornell University, Ithaca, U.S.A. 2010 \\ Bachelor of Technology, S.R.M. University, Chennai, India 2009 \\ A dissertation presented to Ryerson University \\ in partial fulfillment of the requirements for the degree of \\ Doctor of Philosophy in the Program of Mechanical and Industrial Engineering \\ Toronto, Ontario, Canada \\ (C) PriyathaPremnath 2015
}




\section{Author's Declaration}

I hereby declare that I am the sole author of this dissertation. This is a true copy of the dissertation, including any required final revisions, as accepted by my examiners.

I authorize Ryerson University to lend this dissertation to other institutions or individuals for the purpose of scholarly research

I further authorize Ryerson University to reproduce this dissertation by photocopying or by other means, in total or in part, at the request of other institutions or individuals for the purpose of scholarly research.

I understand that my dissertation may be made electronically available to the public. 


\title{
Abstract \\ Bio-functionalization of silicon and its applications in mammalian and cancer cell manipulation and proliferation
}

\author{
Doctor of Philosophy \\ 2015 \\ Priyatha Premnath \\ Mechanical and Industrial Engineering \\ Ryerson University
}

Currently fabricated bio-matrices lack important characteristics such as nanometer scale, 'bumpy' morphology and an interlinked structure. Therefore, cells cultured on such matrices may not truly represent phenotypes of cells grown in the natural environment. This thesis deals with the synthesis of a three dimensional nanofibrous silicon matrix that is interlinked and possesses a 'bumpy' structure that mimics the natural extra cellular matrix. This silicon matrix can be tailored to suit applications of cell proliferation and manipulation.

Cell-biomaterial studies show that osteoblasts and fibroblasts proliferated by $300 \%$ on three dimensional nanofibrous matrix compared to virgin silicon. To induce controlled cell proliferation, the addition of gold to the silicon matrix was perceived. The phase of gold was altered and combined with silicon forming a unique hybrid structure that prevented the growth of cells in areas of increased gold concentration. Increased gold concentration indicated lower adhesion forces and reduced zeta potentials which consequently lead to decreased cell growth. In addition, the interaction of cancer cells with the three dimensional silicon and gold-silicon hybrid nanofibrous network was studied. Results indicate a 96\% reduction in cancer cells compared to 
virgin silicon. The reduction in cells is attributed to- different phases of silicon and silicon oxides in nanoparticle form, the encapsulation of cells by the nanofibers and apoptosis of cells owing to nanoparticles entering cells passively.

To control the growth of cells, silicon surface bio-functionalization was performed to study manipulation of mammalian cells such as fibroblasts as well as cervical and breast cancer cells. The manipulative property is attributed to a mixture of phases of silicon and silicon oxides as well as varied crystal orientations of silicon. It is hypothesized that the mixtures of phases on the substrate alter its surface morphology and consequently induce cell manipulation. Therefore, laser irradiated bio functionalized silicon and its nanostructures are a versatile material for biomedical applications. Based on the process of bio functionalization, both proliferation and cell control and manipulation was achieved in this thesis. 


\section{Acknowledgements}

I express utmost gratitude to my supervisor Dr. Krishnan Venkatakrishnan who constantly supports and encourages me in every aspect. I am amazed by his zeal and passion for research and hope to emulate this in my work. I would also like to thank my co-supervisor Dr. Bo Tan who helped me understand the physics behind laser fabrication and spent long hours revising my work. She has also been a constant source of encouragement. I have been very fortunate to have supervisors who demonstrated faith in my potential and enthused me to aim higher.

I thank Dr. Hua Lu and Dr. Ziad Saghir who helped in refining my thesis with their valuable comments.

My deepest gratitude to my ever so patient husband Gopinath Palanimanickam who has constantly supported and motivated me. He has patiently listened to many hours of me discussing research, both positives and negatives. He has been there to share my joy and lighten my sorrows. He compromised more than what I could expect in every which way. I am grateful for having him in my life and am forever indebted to him.

I would like to thank my loving parents, Latha and Capt. P. Premnath who have supported and motivated me throughout. My father has always wanted the best for his daughters and has done more than what he could to help us accomplish our dreams and aspirations. He always believed in me and my abilities. My long daily conversations with my mother helped me put things in perspective. Her words of encouragement and support kept me grounded and positive. I would like to thank my sister Preseedha Premnath who has been my source of inspiration. We both have kept each other company during our darkest and brightest times. She has been my source of joy throughout this endeavour. Her accomplishments at such a young age has been a tremendous motivating factor. I also thank my parents-in-law and sisters-in-law for always believing in me and showering me with love and affection.

Last but not least I would like to thank my friends who helped me fuse work with pleasure that enabled me to approach my research with a peaceful and fresh perspective. Pooja Thakur, Mathew Jacob and Mathew Denny kept me sane during my doctoral studies. I would also like to thank present and past lab mates especially Amirkianoosh Kiani and Amirhossien Tavangar who 
introduced me to life at Ryerson and helped me acclimatize. My sincere gratitude to the hardest working interns Prasath Mahendrarajah, Nabila Abraham and Dhruv Patel who spent hours working on statistical analysis for my research. Ryerson University played an integral role during my studies especially the staff at the Mechanical and Industrial Engineering Department and for that I am grateful.

Finally, I thank the Almighty for paving the path for my success. 


\section{Dedication}

I lovingly dedicate this dissertation to my husband, parents and sister. Without them, there is no me. 


\section{Table of Contents}

Author's Declaration .......................................................................................................... ii

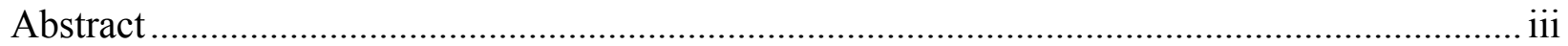

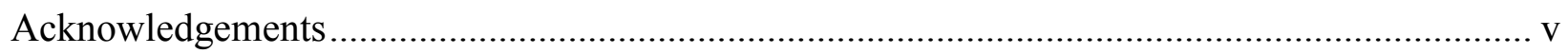

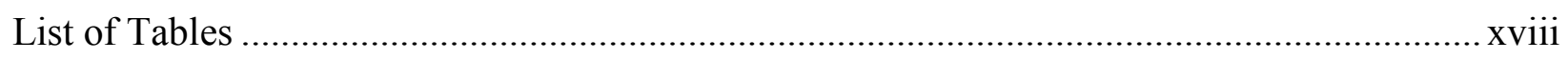

List of Abbreviation ...........................................................................................................

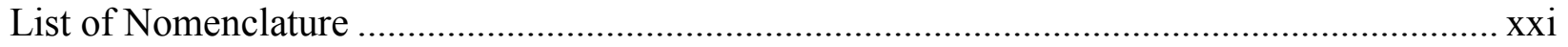

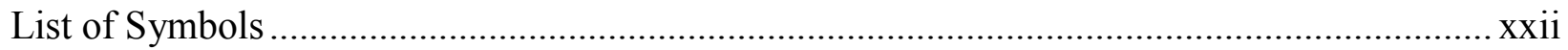

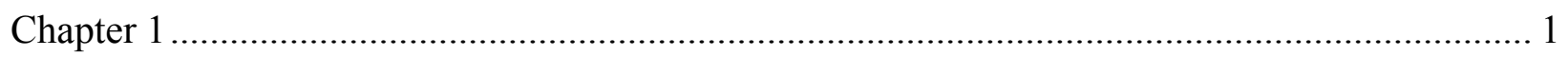

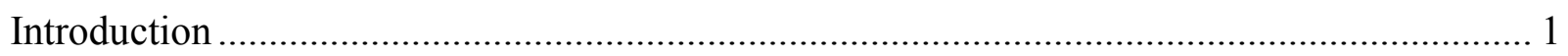

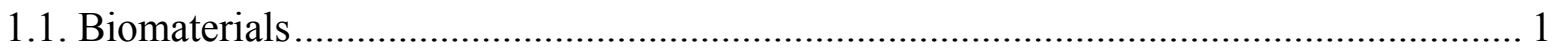

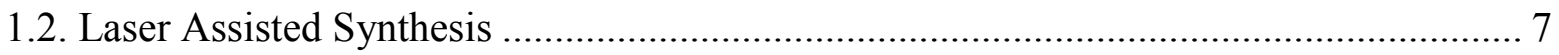

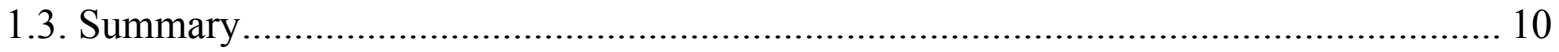

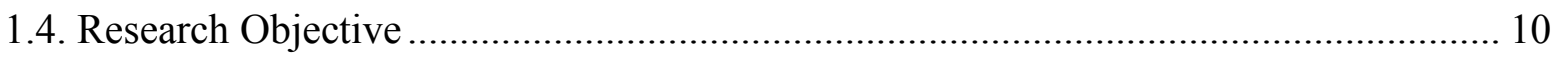

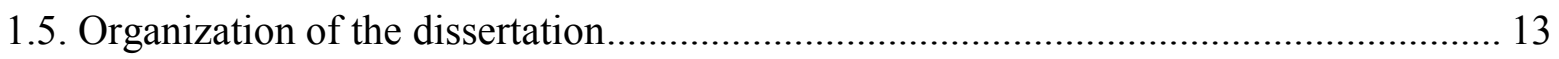

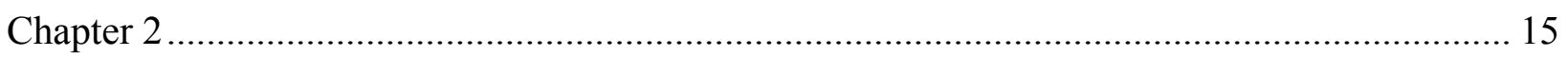

Proposed novel method to mimic natural extra cellular matrix with advantages over previously

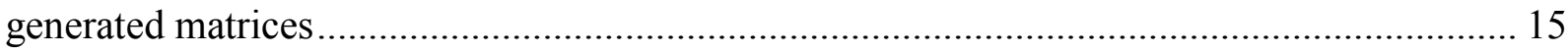

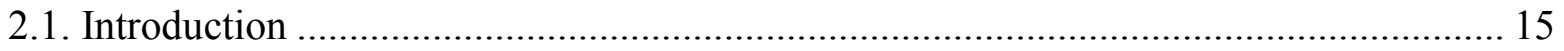

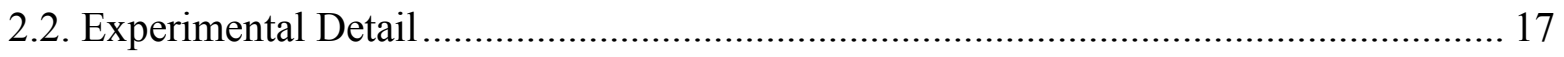

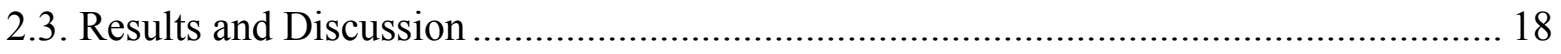

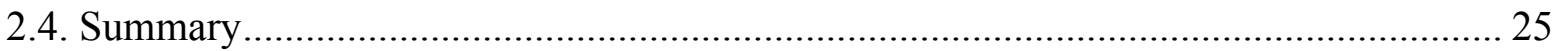

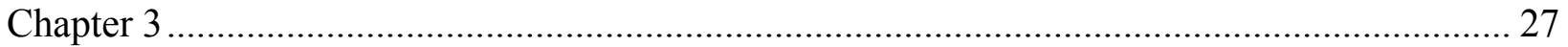

Biodegradability of bioactive interlinked nanostructures and osteoblast interction studies ..... 27

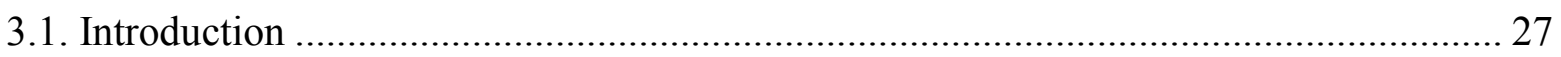

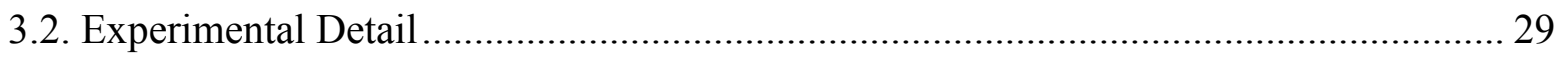

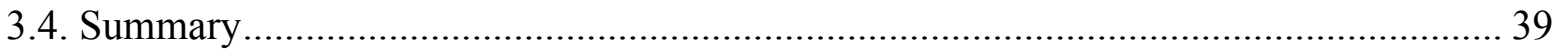

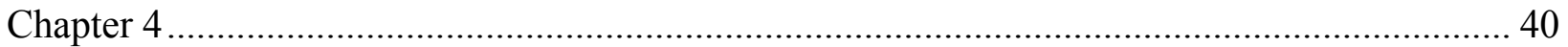

Optimization of the functionalization of bioactive three dimensional nanofibrous network with

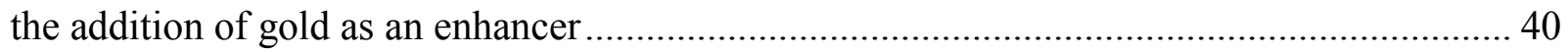

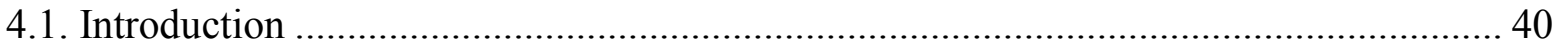




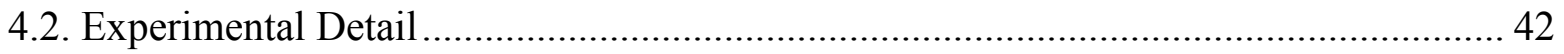

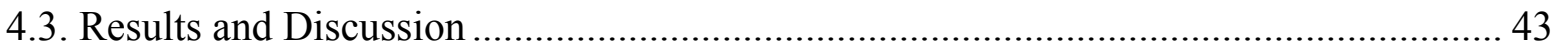

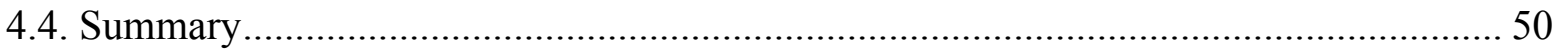

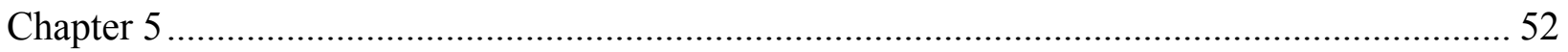

Programming cancer through phase transformed functionalized silicon based biomaterials ... 52

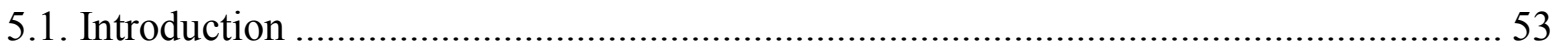

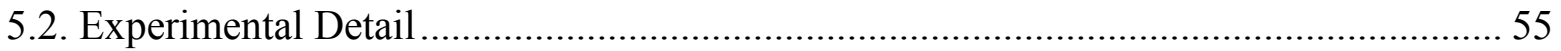

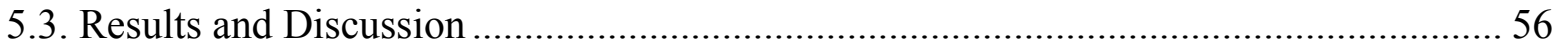

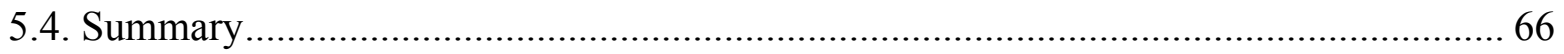

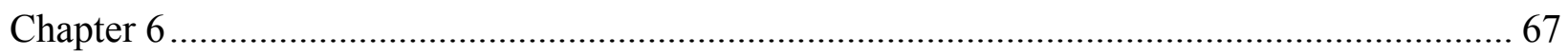

Cancer cell interaction studies with functionalized phase transformed silicon ........................ 67

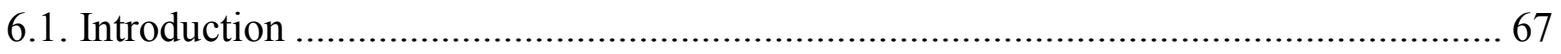

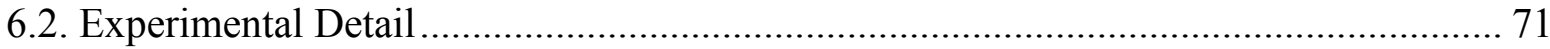

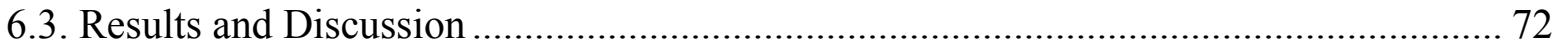

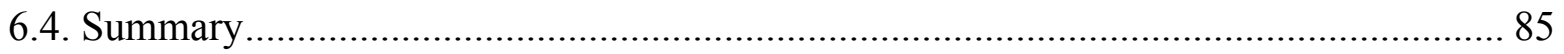

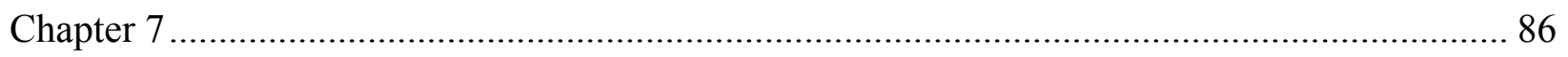

Engineering next generation functionalized multi-phase silicon/silicon oxide nano biomaterials

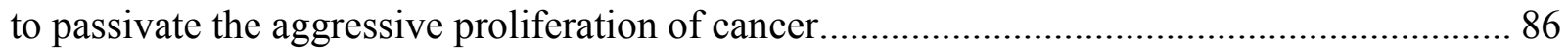

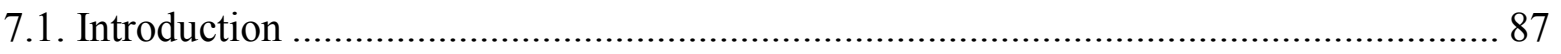

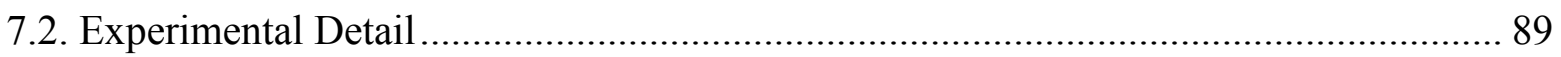

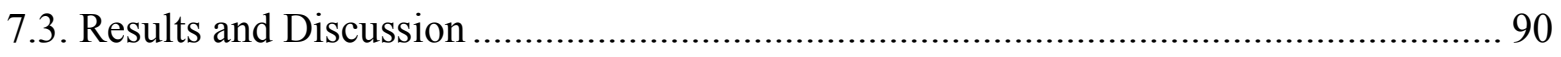

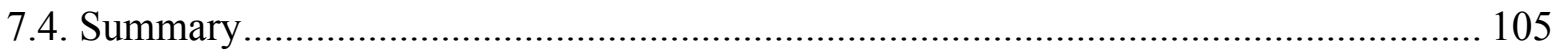

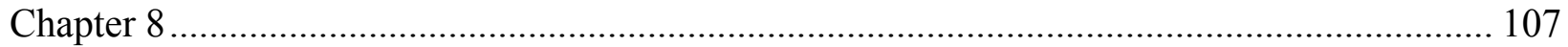

Next Generation bio-functionalized rare phased gradiated gold-silicon/silicon oxide nanostructured hybrid biomaterials to deactivate cancer cell growth.......................................... 107

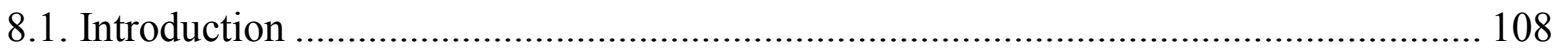

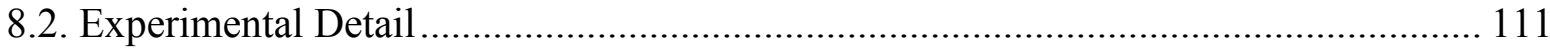

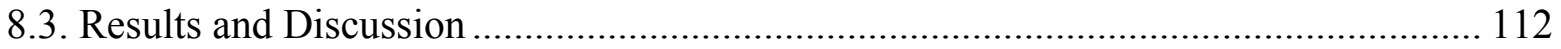

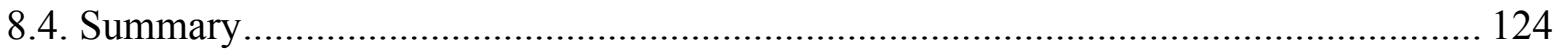

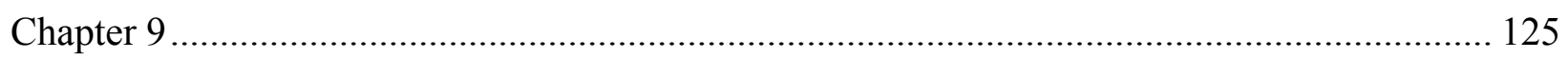

Summary and Future Research …………................................................................ 125

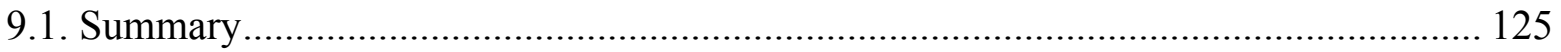

9.2. Future Research ………………………………................................................ 127 


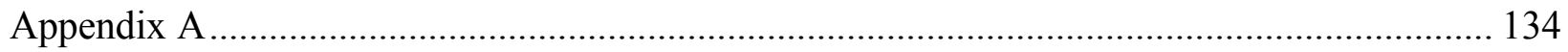

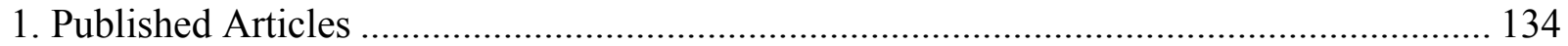

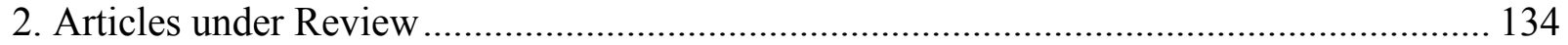

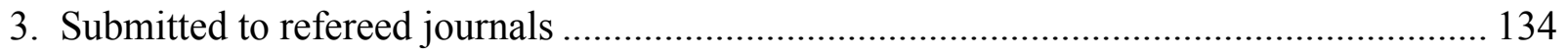

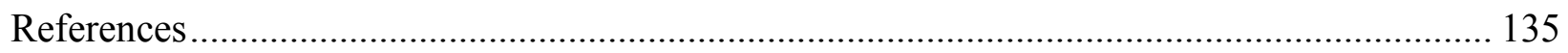




\section{List of Figures}

Figure 1-1: SEM image of collagen fibers (Courtesy[11]) ................................................ 1

Figure 1-2: Electrospinning set-up (Courtesy [12])................................................................. 2

Figure 1-3: Process of polymer demixing of two polymers (Courtesy [17]) ............................. 3

Figure 1-4: Process of phase separation (Courtesy [21] ) ................................................... 4

Figure 1-5: Cell elongation and alignment control due to micropatterning 3D hydrogels

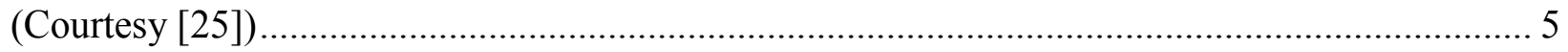

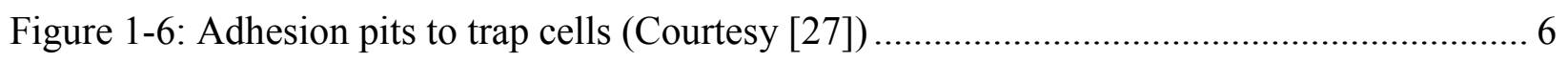

Figure 1-7: Process of microcontact printing (Courtesy [28]) ............................................. 7

Figure 1-8: Fabrication using microcontact printing and transfer sheet process (Courtesy [30]) .. 7

Figure 1-9: Pulsed laser deposition setup (Courtesy $([31])$.................................................... 8

Figure 1-10: Laser induced varying roughness leading to different surface energies and hence

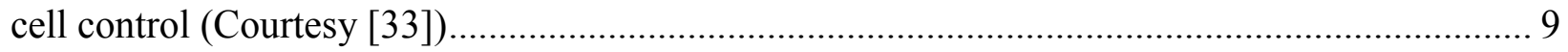

Figure 1-11: Controlled growth of cells on laser ablated polymers (Courtesy [36]).................. 9

Figure 2-1: (a-b) SEM image of silicon nano network (c) TEM of silicon nanoparticles that combine to become silicon nano-network (d) Cross sectional SEM of silicon nano network

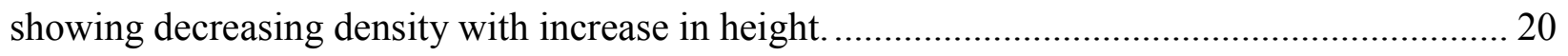

Figure 2-2: Zeta potentials Vs deposition thickness, $0 \mu \mathrm{m}$ thickness being the control sample... 21 Figure 2-3: (a) force curve from a plain silicon surface (the red lines signify approach of tip towards the surface and blue lines signify retraction from surface) (b) force curve from nanonetwork deposition (c) Adhesion forces and deposition thickness. Data at 0 is control silicon... 22 Figure 2-4: SEM micrographs of hydroxyapatite formation on silicon nano-network after (a) 3 days and (b) 10 days. EDS showing spectral analysis of silicon samples immersed in SBF for 3

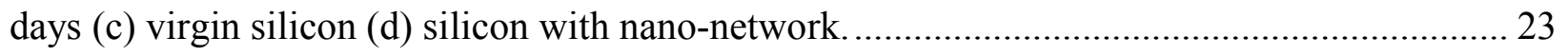

Figure 2-5: Increase in proliferation of NIH3T3 fibroblast cells as deposition thickness increases.

Figure 2-6: (a) SEM of fibroblasts on silicon nano-network formed at 10ms (b-c) Fibroblasts with multiple filopodia spreading out indicating strong adhesion and migration. 25 
Figure 3-1: (a) Possible scaffold shapes that could be generated from $\mathrm{MHz}$ femtosecond laser synthesis (b) Required areas that have silicon nanofibrous network (c) Magnified image showing structure of the nanofibrous network by aggregation of nanoparticles (d) TEM of the nanofibrous network. 31

Figure 3-2: EDS analysis of the silicon nanofibrous network revealed silicon oxide nanoparticles. (a) TEM of silicon nanofibrous network (b) Percentage of oxygen present (c) Percentage of silicon present. 31

Figure 3-3: The degradation rate of the silicon nanofibrous network against different time periods is plotted. The inset represents a cross section of the silicon nanofibrous network that contributes to the rate of degradation. It shows decreasing density and decreasing pore size up to down. 33

Figure 3-4: Various shapes of cells produced after 24 hours of seeding. (a) The circles indicate cells that have grown perpendicular to the surface (b) Multiple cells growing three dimensionally (c) Cells growing at different depths (d) Cells able to traverse across heights of $>100 \mu \mathrm{m}$ migrate and proliferate.

Figure 3-5: (a) A fibroblast that is rounding to divide. (b) The filopodia of the fibroblast interacting with the nanofibrous network. (c) The lamellae extending to adhere to a different area. (d) A fibroblast spreading out to adhere and grow on the silicon nanofibrous substrate..... 35 Figure 3-6: (a) Arrows pointing out the microvilli on osteoblasts (b) Lamellae of the osteoblasts extending with filopodia further extending out (c) A clump of osteoblasts, where the thickness of silicon nanofibrous was higher (d) Filopodia of the osteoblasts inteteracting with the biomaterial surface.

Figure 3-7: Illustration of cell-biomaterial interaction of microscale (a) compared to nanoscale (b). Nanoscale dimensions show superlative interaction 38

Figure 3-8: (a) Effect of particle size on cell proliferation and (b) Effect of pore size on cell proliferation. 38

Figure 4-1: Illustration of the gold-silicon Vs Silicon nanofibers synthesis and application....... 44 Figure 4-2: SEM image of a three dimensional porous alloy like hybrid gold-silicon nanostructure and plain silicon nanostructure. (c) TEM of gold-silicon nanostructure. The red arrows indicate gold nanoparticles or gold-silicon nanoparticles. (d) SEM micrograph of plain silicon nanostructure. 
Figure 4-3: (a) TEM image and the corresponding EDX graphs of hybrid gold-silicon (b) TEM of hybrid gold-silicon fabricated at higher power demonstrating lower percentage of gold (c) TEM of hybrid gold-silicon fabricated at lower power demonstrating higher percent percentage of gold to silicon. Darker shades show gold nanoparticles. 47 Figure 4-4: The XRD micrographs depict the material composition of the gold-silicon hybrid nanostructures fabricated at (a) 78\% gold (b) 35\% gold

Figure 4-5: (a) The adhesion forces of fabricated gold-silicon alloy like hybrid nanostructures shows higher percentage of gold has lower adhesion force. The inset images show representative adhesion curves at each data point (b) The zeta potentials show a similar trend to the adhesion forces where higher percentage of gold has lower zeta potentials. 48 Figure 4-6: (a) SEM image of a fibroblast growth on gold-silicon alloy like hybrid nanostructure. (b) Growth of fibroblast on plain silicon nanostructure. The interaction of the cell with the nanofibers is seen on the magnified image on the right. (c) effect of concentration of gold in gold-silicon alloy like hybrid on fibroblast and osteoblast proliferation (d) cell adhesion morphology on gold-silicon and plain silicon nanostructures. ............................................... 50 Figure 5-1: Illustration fabrication and application of phase transformed cell repellant zones.... 54 Figure 5-2: EDX images of phase transformed zones (A) At 8.76MW (B) 1.31 MW. The yellow arrow shows primary transformed zone and green star denotes secondary transformed zone. (C) AFM micrograph in height contrast (D) AFM micrograph in phase contrast mode (E) micro

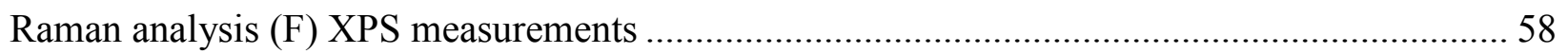
Figure 5-3: Illustration of applications of substrate phase transformation of silicon ................. 61 Figure 5-4: (A-C) Cell repelling phase transformed zones. Red arrows at (A) Represent the cell repelling zone (B) Represent broken adhesion points close to repelling zone and several filopodia away from zone (C) Represents curved cell growth with poor adhesion. (D-F) Cell directionality. The red arrows at (D-F) represent bending of cells along the zone.

Figure 5-5: (A-C) Cell channeling with in a directional fashion. The red arrows at (A-C) Represent channeling of cells along the zone. (D-F) Single cells trapping combining primary and secondary phase transformed zone.The red arrows at (D-F) Represent trapping of cells (F) Magnified image of filopodia interaction with phase transformed and plain silicon.

Figure 5-6: EDX micrographs after cell culture at 8.76 MW (A-B) and 1.31MW (C-D). The images indicate cell growth in areas away from primary phase transformed zone. 
Figure 5-7: Fluorescence microscopy images of actin and nucleus position of cells on primary and secondary phase transformed zones. (A-B) Control/plain silicon (C-D) Cells being repelled from primary phase transformed zone and aligning along secondary phase transformed zone (GH) Cell channeling in a single direction (I-J) Single cell isolation. 64 Figure 5-8: Graph illustrating the correlation between pulse duration and width of cell repellent zone.

Figure 6-1: Synthesis and application of programmable functionalizedsilicon surfaces. The SEM images of various shapes present the versatility of the ultrafast pulse laser synthesis method. The figure depicts an overall mechanism of fabrication and application of phase transformed functionalized silicon in various areas. 70 Figure 6-2: Illustration of pulse-silicon interaction and causative changes in silicon (A) Ratio of silicon to oxygen at different pulse widths (B) micro-Raman spectra of functionalized phase transformed silicon at different pulse widths (C) EDX in mapping mode reflecting oxygen and silicon content on functionalized zone (D) XRD micro spectra and (E) XPS analysis of functionalized phase transformed silicon. 76 Figure 6-3: Cell-functionalized material interaction after seeding for (A) 24 hours and (B) 48 hours. Scanning electron microscopy as well as fluorescence microscopy images showing cell growth and repelling at each functionalization parameter is presented. 78 Figure 6-4: Width of functionalized zone with increase in duration of pulses. The SEM images are representative images at different pulse widths and the red arrows indicate functionalized phase transformed zones. 79 Figure 6-5: Analysis of lamellipodial and filopodial growth on control silicon as well as functionalized phase transformed silicon. (A-C) HeLa cells cultured on control silicon (D-E) Hela cells cultured near functionalized zones and properties of lamellipodia on such zones (G-I) HeLa cell elongation along functionalized zone (J-K)The breaking up of lamellipodia close to functionalized zones. 81

Figure 6-6: Fluorescence images of HeLa cells stained to reveal actin cytoskeleton and nucleus. (A-C) Cells growing on control silicon in a random fashion. (D) Low frequency and short pulse width after 48 hours of growth presenting cell repelling from functionalized zones(E) Cell channelling, cells growing in the same direction after 48 hours, low frequency and short pulse width (F) Filopodial extensions sensing away from the functionalized zone at high frequency and 
short pulse width after 24 hours of growth (G) Focal adhesions forming at points away from functionalized zones at 48 hours, mid frequency and long pulse width $(\mathrm{H})$ Formation of stress fibers in cells growing near functionalized zones at 48 hours, mid frequency and long pulse width. .83

Figure 7-1: Illustration of mechanism of formation of multi-phase silicon/silicon oxide nanostructures potential applications.

Figure 7-2: (A-B) Magnified and zoomed SEM images of multi-functionalized nanostructured silicon (C-D) TEM images showing various nanoparticle sizes (E-H) SEM micrographs of varying nanostructure density of multi-functionalized nanostructured silicon. 94

Figure 7-3: (A) SEM micrograph depicting ultrashort pulsed laser-material interaction zones. The magnified images show the nanostructure density at point of interaction and away (A1) Oxygen content (A2) Silicon content (B) Raman spectroscopy graphs at varying pulse durations and different phase concentrations (C) XRD graphs at different phase concentrations. 95 Figure 7-4: (A) SEM micrograph depicting ultrashort pulsed laser-material interaction zones. The magnified images show the nanostructure density at point of interaction and away (A1) Oxygen content (A2) Silicon content (B) Raman spectroscopy graphs at varying pulse durations and different phase concentrations (C) XRD graphs at different phase concentrations..............96 Figure 7-5: Number of cells on different phases of nanostructures as well as different pulse generated nanostructures. Comparison is made between cells on high density nanostructures and control or virgin silicon at (A) 24 hours and (B) 48 hours. (C-D) SEM images of HeLa cells on control or virgin silicon (E-F) Fluorescence microscopy images of HeLa cells on control or virgin silicon (G-H) SEM images of HeLa cells on high density nanostructures (I-J) Fluorescence microscopy images of HeLa cells on high density nanostructures. All images are of cell growth at 48 hours. 98 Figure 7-6: (A) The length along the major axis of HeLa at 24 and 48 hours on crystalline rich phases on varying densities of nanostructures (B) The length along the major axis of HeLa at 24 and 48 hours on amorphous rich phases on varying densities of nanostructures (C-E) SEM images of cell growth at 48 hours on crystalline rich phases (F-H) Fluorescence microscopy images of cell growth at 48 hours on crystalline rich phases (I-K) SEM images of cell growth at 48 hours on amorphous rich phases (L-N) Fluorescence microscopy images of cell growth at 48 hours on amorphous rich phases. 100 
Figure 7-7: Mechanism of cell death. (A-B) SEM images of HeLa on control virgin silicon after 48 hours (C-D) Fluorescence microscopy images of HeLa on control virgin silicon after 48 hours (E-F) SEM images of HeLa on amorphous rich nanostructures after 48 hours (G-H) Fluorescence microscopy images of HeLa on amorphous rich nanostructures after 48 hours. 102 Figure 7-8: Measuring nucleus over low and high density nanostructures. The graph shows reduced nucleus size at high density nanostructures. 103

Figure 7-9: Graph depicts the lamellipodia length of control and high density nanostructures. (A, B, F, J) Illustrates a cell growing on nanostructures as well as control area illustrating lamellipodial adhesion on control silicon and less adhesion of nanostructures (C-F) Cell growth on control silicon (G-I) Cell growth in nanostructured silicon. 104

Figure 7-10: Percentage reduction of cells on deposited nanostructures. Significant reduction in number of cells is observed at high density of deposited nanostructures 105 Figure 8-1: Illustration of generation of 'anti-cancer' gold-silicon nano hybrids and its applications

Figure 8-2: (A) SEM of gold-silicon nano hybrids (B) TEM showing gold-core silicon-shell structure (C-D) Varying densities of nano hybrids (E) High density nano hybrids (F) Low density nano hybrids. The figure also illustrates the varying concentration of gold in the nano hybrids (G-H) High gold concentration (I-J) Low gold concentration (K-L) Large gold-cores (M-N) Smaller gold-cores.

Figure 8-3: (A-D) TEM and EDX of gold-silicon nano hybrids (E) XRD micrograph of goldsilicon nano hybrids. 115 Figure 8-4: (A) Graphical representation of cell growth on various densities. Cell growth on (B) High density (C) Medium Density (D) Low Density (E) Illustration of nano hybrid interaction with cell...... 117

Figure 8-5: Relative occurrence of different kinds of cells at (A) 24 hours and at (B) 48 hours. 118

Figure 8-6: (A) Graphical representation of cell interaction at different concentration of gold. (B1-B3 and C1-C3) Representation of endocytosis of nanoparticles in nano hybrids and eventual cell death. 119

Figure 8-7: (A) Interaction of cells at different pulse widths (B-C) Gold rich at 214fs (D-E) Gold rich at $1428 \mathrm{fs}(\mathrm{F}-\mathrm{G})$ Silicon rich at $214 \mathrm{fs}$ (H-I) Silicon rich at $1428 \mathrm{fs}$. 120 
Figure 8-8: Nucleus area illustrating cell health. Short pulse presents smaller nucleus sizes

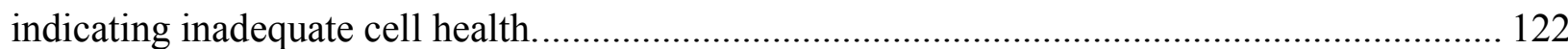
Figure 8-9: Graph depicts number of filopodia on various nanohybrids and control virgin silicon. (A-B) High density nanohybrids (C-D) Low Density nanohbrids (E-F) Control virgin gold.... 123 Figure 9-1: Illustration of silicon nanofibers enveloped by gold or gold-palladium..... 127 Figure 9-2: Influence of gold and gold-palladium and density of nanofibers over 24 and 48 hours.

Figure 9-3: The influence of concentration of gold and gold-palladium on cell growth........... 129 Figure 9-4: Growth of breast cancer cells on gold and gold-palladium enveloped silicon nanofibers at 24 and 48 hours fabricated at short and long pulse...................................... 130 Figure 9-5: Surface patterning of 'anti-cancer' nanostructures and its applications. 132 


\section{List of Tables}

Table 1: Surface temperature on silicon after multiple pulse interaction at various peak powers.

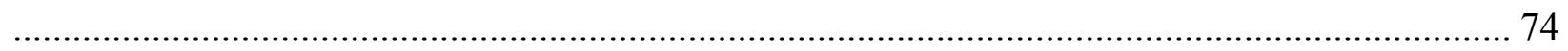




\section{List of Abbreviations}

AFM Atomic force microscopy

BSA Bovine serum albumin

CVD Chemical vapour deposition

DMEM Dulbecco's Modified Eagle Medium

DMSO dimethyl sulfoxide

DPBS Dulbecco's Phosphate-Buffered Saline

ECM Extra Cellular Matrix

EDS Energy dispersive X-ray spectroscopy

fs Femtosecond

HA Hydroxyapatite

HAZ Heat affected zone

HEPS 4-(2-hydroxyethyl)-1-piperazineethanesulfonic acid

MEMSmicroelectromechanical systems

MHz Megahertz pulse

MTT 3-(4,5-Dimethylthiazol-2-Y1)-2,5-Diphenyltetrazolium Bromide

nm Nanometer

ps Picosecond

SBF simulated body fluid

SEM Scanning electron microscope

TEM Transmission electron microscopy

XRD X-Ray Diffraction 
ZP Zeta potential

$\boldsymbol{\mu m}$ Micrometer 


\section{List of Nomenclature}

\begin{tabular}{|c|c|}
\hline Apoptosis & Death of a cell in a normal, controlled fashion \\
\hline Biocompatible & Characteristic of not being harmful to tissue \\
\hline Cytoskeleton & A network of protein filaments that give the shape to a cell \\
\hline Ex vivo & $\begin{array}{l}\text { Condition where tests are performed on a living tissue outside the } \\
\text { body with minimal changes to the natural environment }\end{array}$ \\
\hline Extra cellular matrix & $\begin{array}{l}\text { A collection of molecules that are secreted by cells that provides } \\
\text { structural and biochemical support to the cells }\end{array}$ \\
\hline Fibroblasts & $\begin{array}{l}\text { A type of cell that synthesizes extra cellular matrix and collagen and } \\
\text { has a critical role in wound healing }\end{array}$ \\
\hline Filopodia & Slender cytoplasmic projections that aid in migration \\
\hline Focal adhesion & Strongest point of adhesion by the cell to the substrate \\
\hline In vitro & Tests that take place outside a living organism \\
\hline In vivo & Tests that take place inside a living organism \\
\hline Integrin & $\begin{array}{l}\text { Receptors that form bridges between cell-cell and cell-substrate } \\
\text { interactions }\end{array}$ \\
\hline Lamellipodia & Flattened protrusion of the cell that adheres to the substrate \\
\hline Nucleus & Dense organelle in a cell that contains the genetic material of the cell \\
\hline Osteoblasts & Cell that contribute to bone production \\
\hline Proliferation & Rapid increase in numbers \\
\hline
\end{tabular}




\section{List of Symbols}

$\begin{array}{ll}\mathrm{C}_{\mathrm{p}} & \text { Specific heat, } \mathrm{J}(\mathrm{kgK})^{-1} \\ \mathrm{~F} & \text { Focal length, } \mathrm{mm} \\ \mathrm{f} & \text { Laser frequency, Hz } \\ \mathrm{I}_{0} & \text { Laser energy, } \mathrm{J} \\ \mathrm{k} & \text { Thermal conductivity, W }(\mathrm{mK})^{-1} \\ \mathrm{Neff} & \text { Effective number of pulses } \\ \mathrm{P} & \text { Power, W } \\ \mathrm{r} & \text { Radius, } \mathrm{m} \\ \mathrm{r}_{0} & \text { Diameter of the beam spot, } \mathrm{m} \\ \mathrm{T} & \text { Temperature, } \mathrm{K} \\ \mathrm{T}_{\mathrm{ref}} & \text { Reference temperature, } \mathrm{K} \\ \mathrm{t} & \text { Time, } \mathrm{s} \\ \mathrm{t}_{\mathrm{p}} & \text { Pulse width (duration), } \mathrm{s} \\ \mathrm{V} & \text { Scanning speed, } \mathrm{m} / \mathrm{s}\end{array}$




\section{Chapter 1}

\section{Introduction}

\subsection{Biomaterials}

Recent years have witnessed an increase in the applications of biomaterials in biomedical areas. However, the functions of biomaterials or the basic question of 'what is a biomaterial' is still undefined. Some researchers believe that materials with pertinence in medical applications, like prosthesis are biomaterials whereas other researchers have a broader definition that includes applications like drug delivery and biosensing[1]. Working with the broad definition, where materials in contact with biomolecules are considered biomaterials, the applications of biomaterials can be categorised into two distinct areas- cell proliferation and cell control/manipulation.

\subsubsection{Cell proliferation}

Cell proliferation is needed to repair, restore or enhance the functions of tissues or organs either in in vivo or ex vivo applications. In the natural environment, cells grow in close contact with the extra cellular matrix (ECM). The ECM is composed of nanofibers that are randomly oriented, interlinked and three dimensional in nature (Figure 1-1)[2]. Several techniques have attempted to mimic this structure in an effort to proliferate cells in an environment similar to the ECM. In this regard, micro and nanostructures such as fibers, grooves, tubes, islands and pores were synthesized [3-10].The fabrication techniques employed to synthesize cell proliferating environments will be discussed in this section.

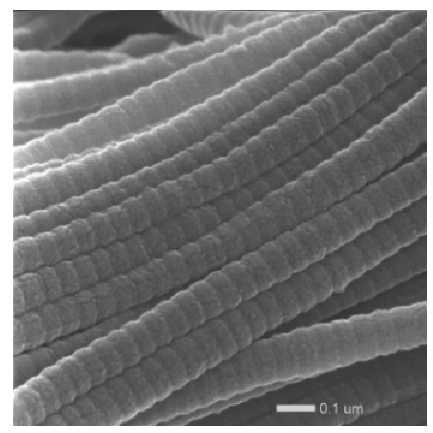

Figure 1-1: SEM image of collagen fibers (Courtesy [11]) 


\subsubsection{Electrospinning}

Electrospinning is a versatile technique that is employed to generate fibers of varying diameters for applications in cell proliferation. The set-up consists of a high-voltage power supply, a spinneret and a collector (Figure 1-2). A polymeric solution is stored in the syringe which is moved to the needle via a syringe pump. When voltage is applied to the needle, the polymeric solution forms a conical shape and when critical voltage is exceeded the polymeric solution ejects from the needle and deposits on the conductive collector[11].Lao et al. employed electrospinning technique to generate Poly(lactase-co-glycolide) (PLGA) nanofibers with nano hydroxyapatite particles[12]. They measured the interaction of the nanofibers with mouse osteoblasts. They observed that the proliferation of osteoblasts on nanofibers with and without the nano hydroxyapatite was the same. However, the health of cells (in terms of attachment) was more on nanofibers that had nano hydroxyapatite. This in turn shows that cells prefer nanofibers with a degree roughness on them to enhance healthy growth of cells. A similar study by Nandakumar et al. introduced surface modification to the polymer nanofibers by oxygen plasma method for enhanced cell proliferation[13]. Therefore, it is ascertained that electrospinning is a viable method to produce nanofibers. However, there are certain drawbacks that are to be addressed. The chemistry of the nanofibers cannot be changed and requires additional steps to change its surface chemistry. Further, due to the smooth nature of its fibers it does not mimic collagen fibers. Moreover, the nanofibers are not interlinked like the natural ECM. In addition, electrospinning is limited in the materials that can be employed to form nanofibers.

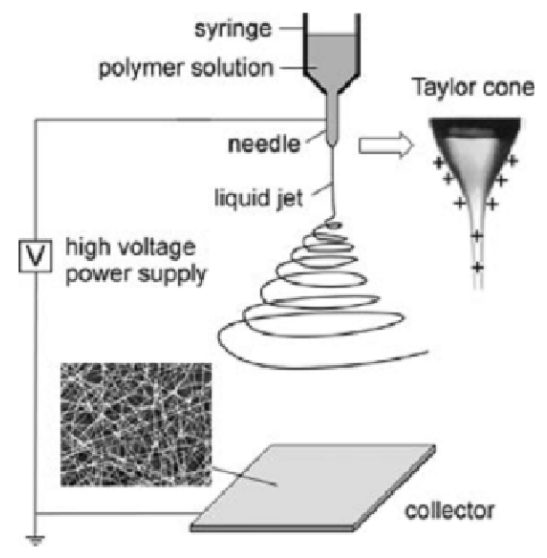

Figure 1-2: Electrospinning set-up (Courtesy [12]) 


\subsubsection{Polymer Demixing}

This is a simple method to create cell proliferative substrates. This method is based on phase separation of two polymers while being spin coated (Figure 1-3). Dalby et al. employed this technique to generate islands of varying heights and studying cell response on it. It is observed that the island with a height of $13 \mathrm{~nm}$ was most effective in cell proliferation and cell health[14]. In another study Lim et al. produced pits of varying depth through polymer demixing. They were able to regulate focal adhesions based on the depths of the pit and hence enhance proliferation[15].While this method presents a simple and versatile way of producing cell proliferative substrates they are limited in the features that they can produce (pits, islands, and ribbons). Moreover, like the title suggests only polymers can be fabricated in this way. More importantly, there is low control over the shape being fabricated.

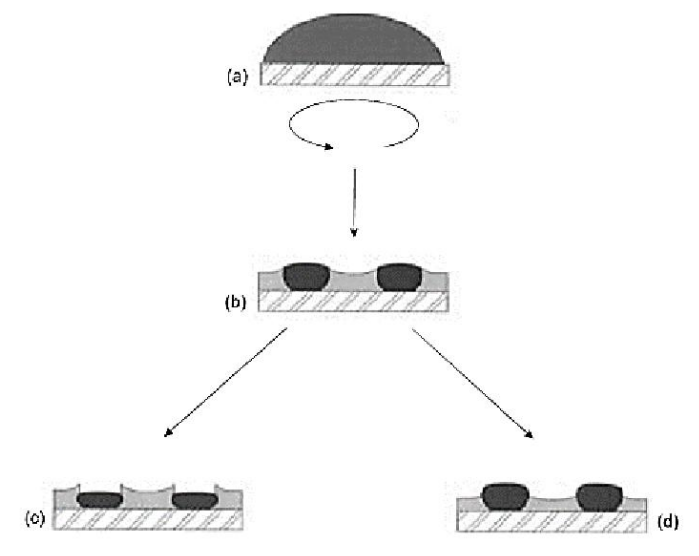

Figure 1-3: Process of polymer demixing of two polymers (Courtesy [17])

\subsubsection{Photolithography}

Photolithography is one of the primary methods of fabrication of ordered structures. A photoresist is coated on a silicon waver. Near-UV wavelength is used to selectively pattern silicon based on the pattern of the photoresist. Braber et al. achieved micro-grooves using photolithography[16]. The directed growth of rat dermal fibroblasts suggests strong influence of the surface grooves in growth. It was observed that smaller micro grooves provided more adhesion points for cells to grow. This method is severely limited due the wavelength of light used to. Therefore, the structures fabricated are limited to the micron scale. 


\subsubsection{Self-assembly}

This method involves the use of polymers that form a nanofibrous scaffold based on selfassembly. A peptide with hydrophobic sequences moves away from water and is towards each other.Tu and Tirrell have provided a detailed review of the various forms of self-assembly and physics involved [17]. Cai et al. modified the surface of titanium via self-assembly using different functional moieties and observed a change in cell proliferation based on moiety[18]. In addition to its difficult process of fabrication, self-assembly introduces monomers that might be potentially be cytotoxic.

\subsubsection{Phase Separation}

Phase separation is another simple method of preparation of a scaffold like structure. It produces a porous interlinked structure. As shown in Figure 1-4the phase separation involves the dissolution of the polymer followed by gelation and solvent removal[19]. Kim et al. successfully cultured and proliferated osteoblasts for a period of 4 weeks on a phase separated PLLA scaffold[20]. The principal disadvantage of this method is its ability to produce only micron sized structures. Further, only polymers can be employed.

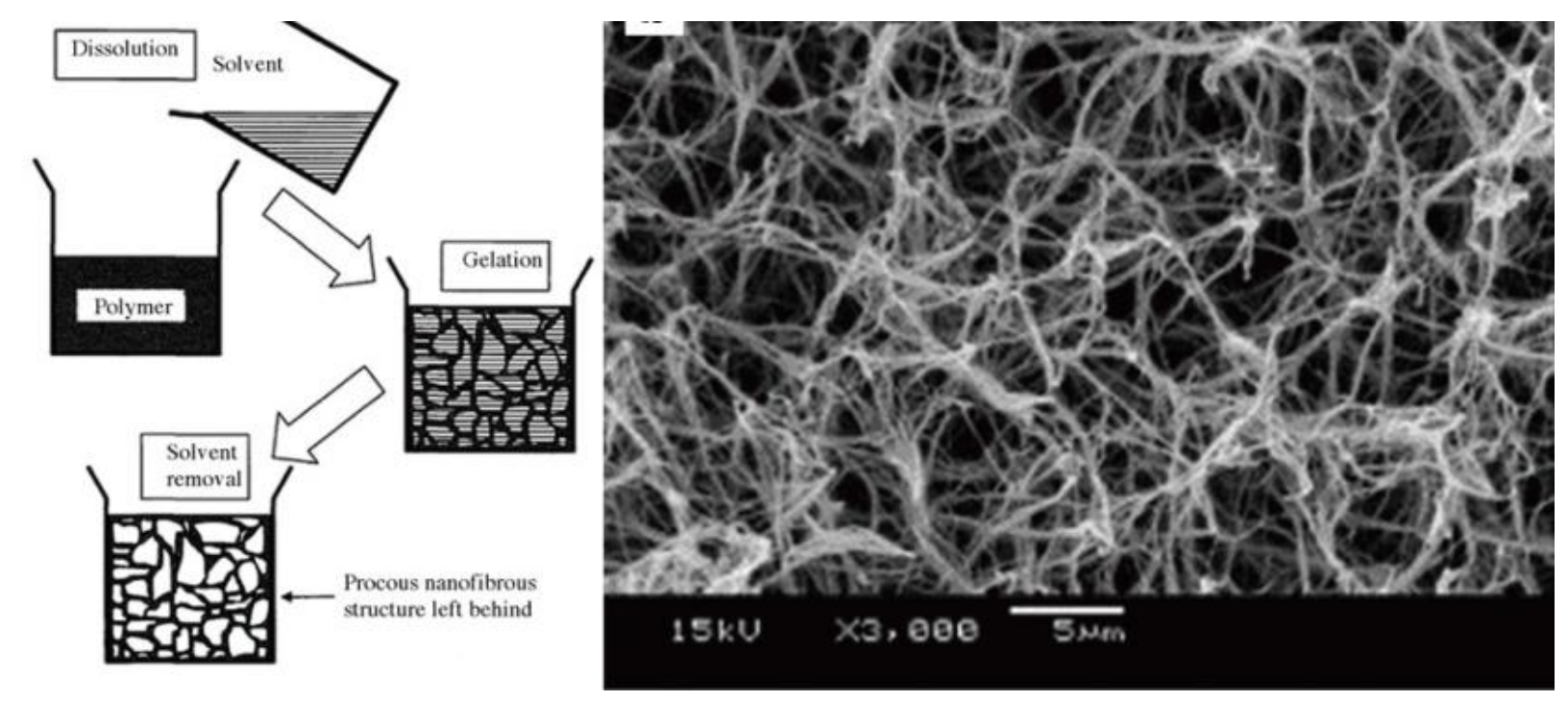

Figure 1-4: Process of phase separation (Courtesy [21]) 


\subsubsection{Cell control and manipulation}

Recent advances in cell-biomaterial interaction studies have led to carefully tailored configurations of biomaterials that can control, manipulate and isolate cells. Like cell proliferation, there are several methods that are employed to create topographical and chemical changes to the surface of the substrate. However, notably, the majority of methods involved controlling cells are chemical based methods. These methods will be discussed in the following section.

\subsubsection{Micropatterning}

This method is a very simple technique of pattering substrates with selective properties. An elastomeric stamp is used to transfer inked patterns to the substrate [21]. Rodriguez et al. explored a micropatterning technique that is able to create cell adhesive, cell non adhesive and controlled adhesive regions on the same substrate [22].In another study, Aubin et al. successfully controlled the alignment and elongation of fibroblast, myoblasts, endothelial and cardiac stem cells using micropatterned 3D hydrogels (Figure 1-5)[23]. The main drawback lies in its limitations in the material that can be patterned, usually polymers.
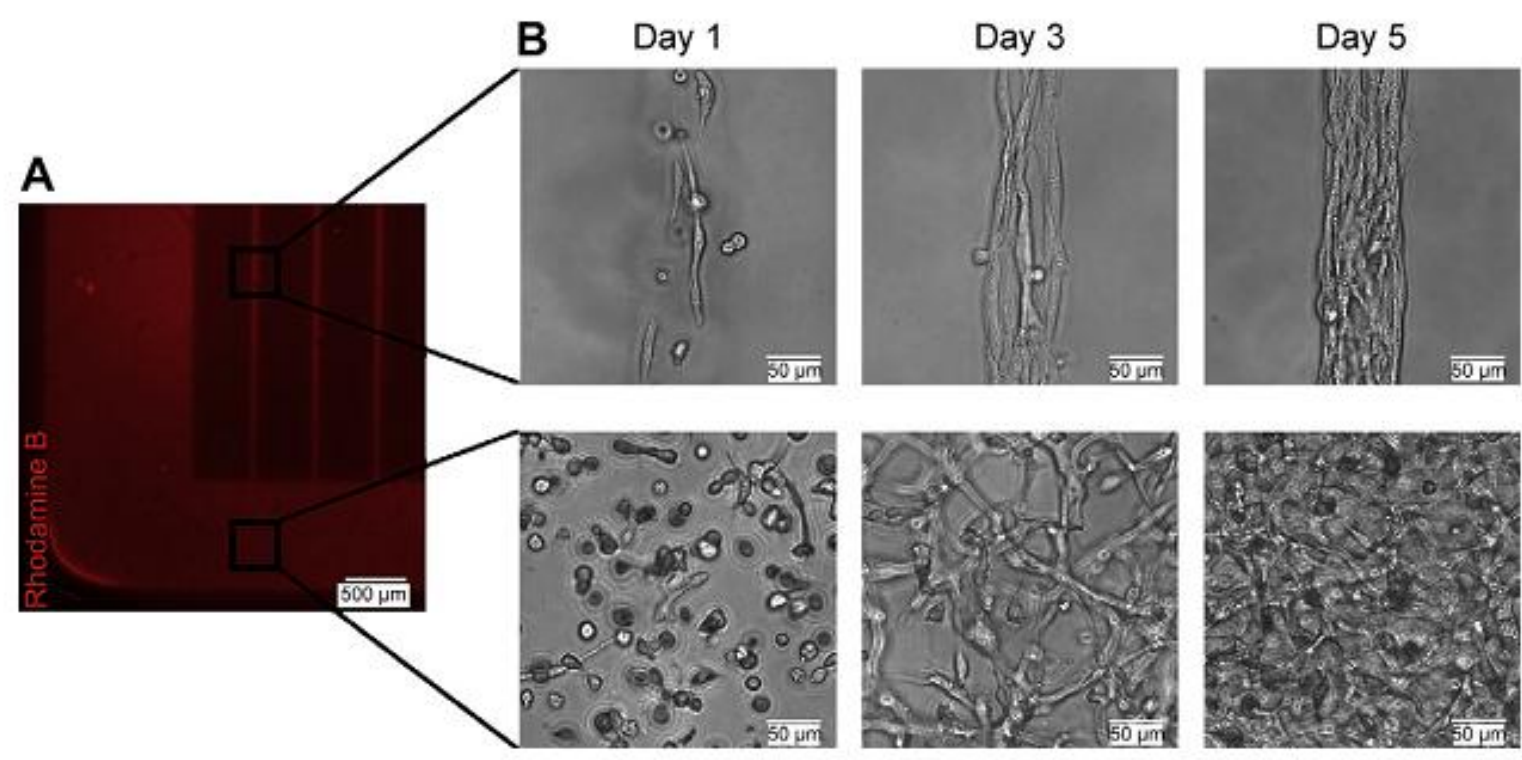

Figure 1-5: Cell elongation and alignment control due to micropatterning 3D hydrogels (Courtesy [25]) 


\subsubsection{Photolithography}

As mentioned before, photolithography uses light to create various structure on a surface. Revzin et al. fabricated micro arrays on PEG-modified glass by employing photolithography that are capable to encapsulating cells on an 'adhesion pad' [24]. A similar study by Koh et al. provided encapsulation of mammalian cells and bacteria in areas on high adhesion and hence controlling the growth of the cells [25]. In both these studies photolithography produced a pit and island-like structure to trap cells within the pits as seen in Figure 1-6. In both the studies, a polymer base is used to selectively adhere to cells by change the surface chemistry of the polymer. As mentioned previously resolution is a primary concern.

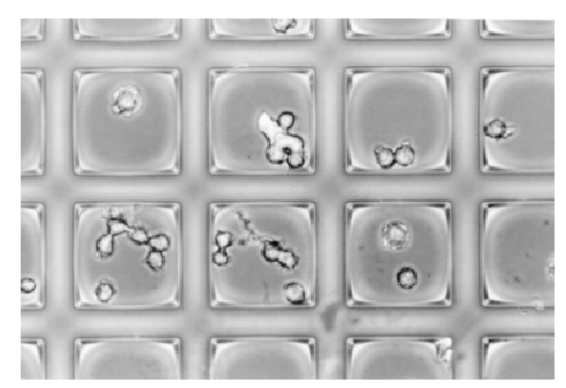

Figure 1-6: Adhesion pits to trap cells (Courtesy [27])

\subsubsection{Micro contact printing}

This method is conventionally used to transfer specific proteins or molecules on to a substrate that is patterned. The molecules attach to the higher areas of the patterned substrate (Figure 1-7)[26]. Microcontact printing was used to achieve graduated lines of different distances and widths[27]. They observed that more than the interval width, the pattern widths influence the orientation and guidance of the Schwann cells. Schwann cells require contact guidance to growth. Hannachi et al. employed microcontact printing and transfer of sheet of cells to coculture two kinds of cells successfully (Figure 1-8)[28]. Despite being a simple and cost effective method, this technique is again limited to polymers. 


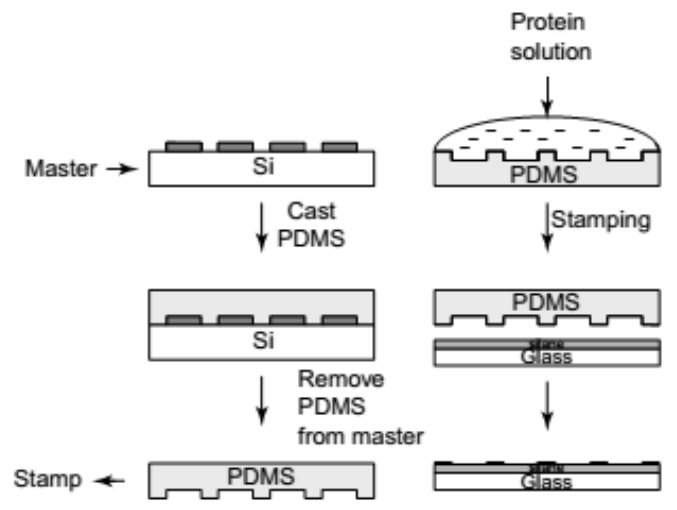

Figure 1-7: Process of microcontact printing (Courtesy [28])

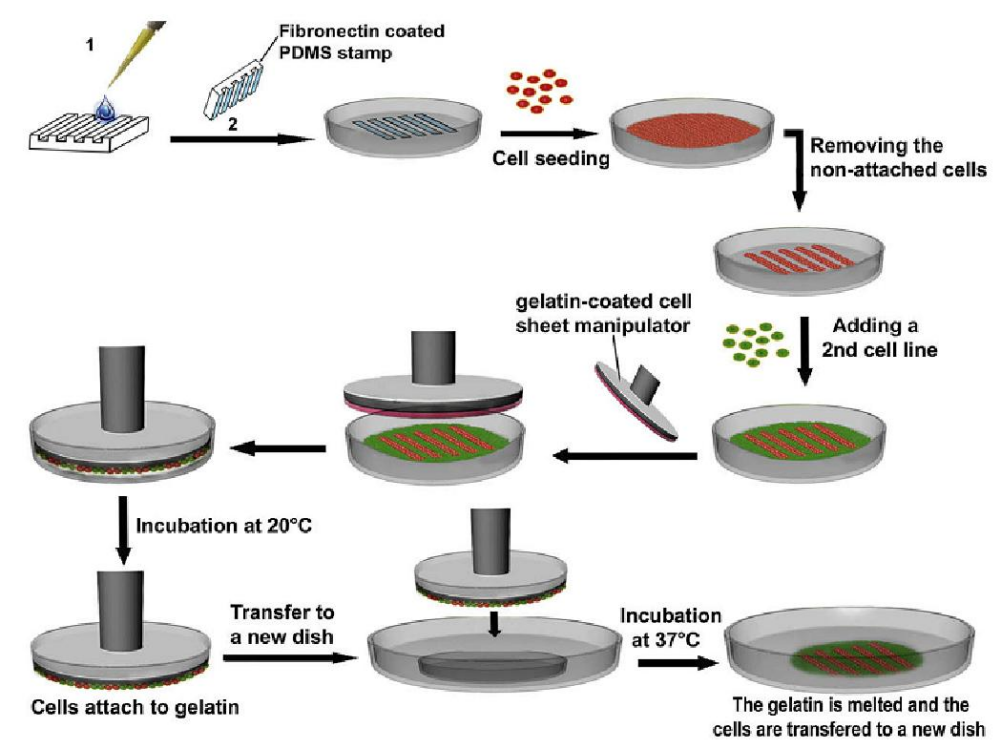

Figure 1-8: Fabrication using microcontact printing and transfer sheet process (Courtesy [30])

\subsection{Laser Assisted Synthesis}

Laser assisted synthesis is a promising candidate for fabrication of biomaterials. The unique fabrication method allows the use of laser to manufacture both cell proliferative and cell controlling or manipulating surfaces. There are several studies that aim to modify the surface of implants to increase its integration in the body. One manner of changing surface characteristics is to add surface coatings. For instance, Zeng and Lacefield used pulsed laser deposition to add hydroxyapatite to the surface of titanium or silicon (Figure 1-9)[29]. They were able to precisely control the composition of the crystals as well as density of crystals. Jonge et al. reviewed 
current methods and surmised that laser assisted deposition had maximum control over the morphology and density of coatings [30].

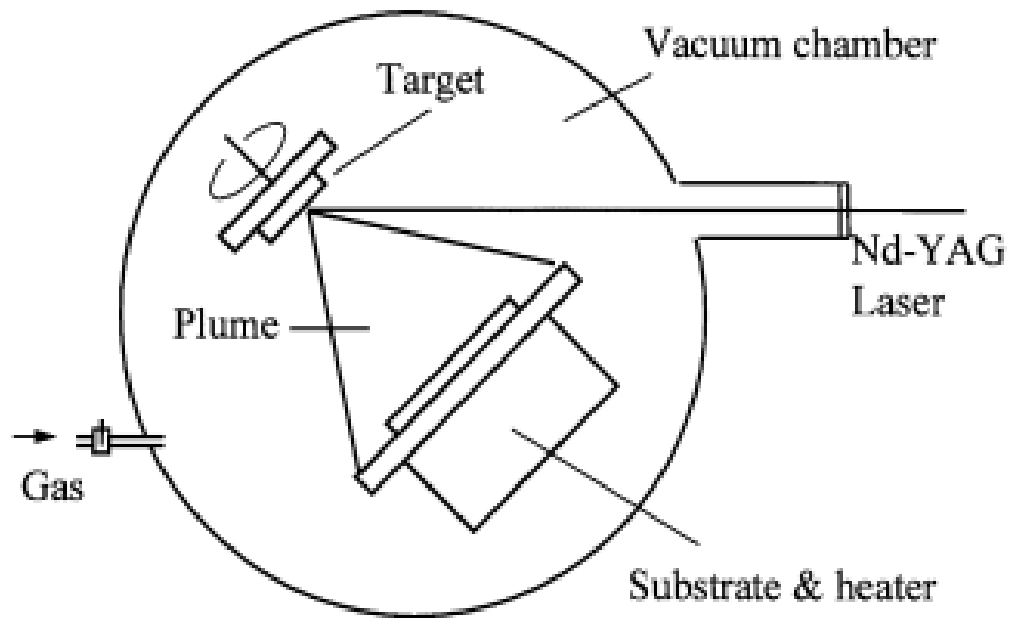

Figure 1-9: Pulsed laser deposition setup (Courtesy ([31])

Ultrafast pulsed lasers have been used to synthesize micro and nanostructures directly on the substrate. Ranella et al. fabricated micro and nano spikes on the surface of silicon [31]. By changing the fluence of the laser, they synthesized different roughness that resulted in different surface energies. Consequently, a change in surface energies resulted in controlling the cell growth. Koufaki et al. fabricated similar spiked roughness of silicon and obtained analogous results[32].

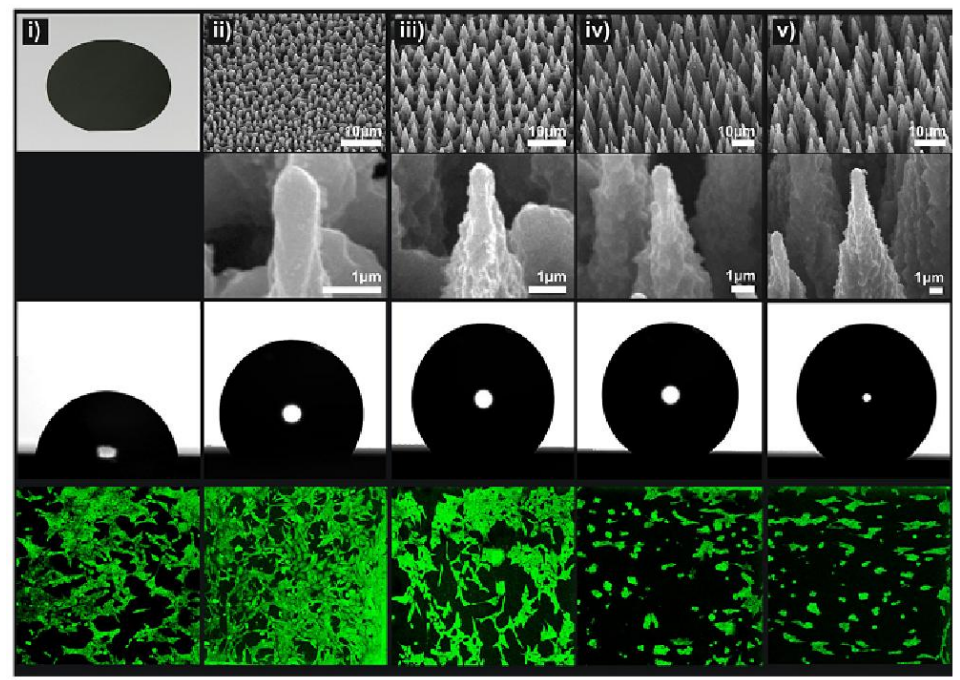


Figure 1-10: Laser induced varying roughness leading to different surface energies and hence cell control (Courtesy [33])

To produce aligned proliferation of cells, Rebollar et al. synthesized curved groves with different periodicity. This encouraged the proliferation of cells in an aligned fashion[33]. Laser has also been employed with other materials such as polymers. Yeong et al. created microchannels on a polymer base using direct laser writing[34]. As seen in (Figure 1-11), the cells on microchannels were limited to the ablated regions compared to control polymer.
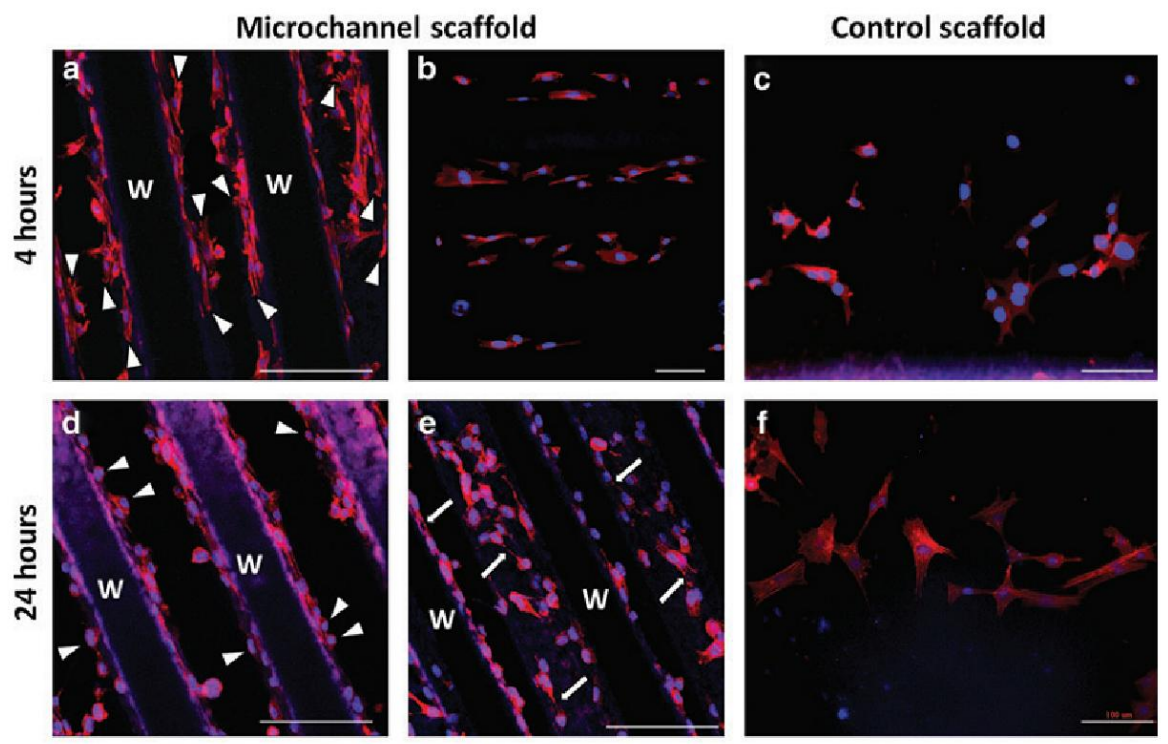

Figure 1-11: Controlled growth of cells on laser ablated polymers (Courtesy [36])

Therefore, it is noted that laser assisted synthesis of biomaterials is a viable and versatile mode. It has several advantages compared to other modes of synthesis. Laser synthesis can be performed on any material. As this research will show, the synthesis of metastable phases is possible. It is possible to deposit structures from one substrate to another target or deposit structures on the same substrate. Laser synthesis offers precise control over the morphology, topology, chemistry and composition of the structures[35].

In this dissertation, femtosecond laser pulsed laser with pulse to pulse separation between micro and nanoseconds is employed to synthesize cell proliferative and cell controlling/manipulating zones. To create a proliferative surface, a nanofibrous matrix on silicon is synthesized. To induce selective cell proliferation, gold was added to the nanofibrous matrix. An increase in gold 
content reduced the growth of cells. Cell control and manipulation is also measured on laser ablated surfaces. Cell control is obtained via sub-surface modification and nanostructured modification of silicon. Laser synthesis allowed for the fabrication of silicon, silicon oxides, gold and gold oxides on the substrate surface.

\subsection{Summary}

Laser assisted synthesis has proven to be a promising candidate in manufacturing of biomaterials. Research in the micro and nano fabrication lab has focussed on the synthesis of three dimensional structures [36-39]. Further, promising preliminary results by Tavangar et al. on the bioactivity and biocompatibility of titanium nanofibrous platforms cemented the research direction for this dissertation[40,41]. The gap in synthesizing natural ECM like three dimensional nanofibrous structure as well as a surface that can effectively control and manipulate the growth of cells is successfully tackled in this dissertation. Through this dissertation, I have fabricated a natural ECM-like interlinked, three dimensional, randomly oriented nanofibrous structure and cell controlling/manipulating substrates that are capable of selectively inducing apoptosis.

\subsection{Research Objective}

Ultrafast pulsed laser synthesis offers promise in the generation of novel biomaterials. This versatile mechanism is capable of selectively modifying the phase of silicon or change its topographical features. The ultrashort pulse laser presents pulses in a separation time of micro to nanoseconds. This distinct characteristic enables the generation unique phases of both silicon as well as silicon oxides.

The main objective of this thesis is to bio-functionalize the surface of silicon using ultrafast pulsed laser synthesis. This in turn will enhance or diminish the inherent proliferative nature of silicon. This will be accomplished through three sub-objectives that individual but related tasks:

Sub-Objective 1: Synthesize a biomaterial that is three dimensional and mimics the natural extra cellular matrix composed of silicon and its oxides.

Task1: Synthesize a three dimensional nanofibrous matrix that is interlinked and mimics that natural extra cellular matrix. Explore ultrafast laser-material interaction and causative factors. 
Task 2: Characterize the morphology of the nanofibrous network and study surface characteristics.

> Scanning Electron Microscopy (SEM), Energy dispersive X-ray spectroscopy (EDX) and Transmission Electron Microscopy (TEM): Characterize morphology, pore size, porosity and interlinking between layers.

$>$ Atomic force Microscopy: Characterize force of attraction of nanofibers synthesized at different laser-material interaction times.

$>$ Zetasizer: Calculate zeta potentials on individual nanoparticles in the nanofibrous matrix.

$>$ Biodegradability: Study rate of degradation of nanofibers in a biological environment to extrapolate stability in vivo.

Task 3: Study interaction of mammalian cells such as fibroblasts and osteoblasts with the developed nanofibrous matrix. Interaction studies will be based on proliferation and adhesion characteristics.

$>$ Proliferation: MTT 3-(4, 5-dimethylthiazol-2-yl)-2, 5-diphenyltetrazolium bromide (MTT) assay will be used to determine cytotoxicity of the nanofibrous matrix and will be an indicator of proliferation of cells.

Adhesion: The adhesion of cells will be studied to determine the health of cells.

Task 4: Study the influence of gold on growth and proliferation of fibroblasts and osteoblasts.

Laser assisted synthesis of gold-silicon hybrid structure. Develop theoretical formation mechanism of gold-silicon hybrid.

$>$ Characterize the surface via SEM, TEM, AFM and zetasizer and compare to silicon nanofibers.

$>$ Study interaction of fibroblasts and osteoblasts via SEM to analyse proliferation and adhesion characteristics.

Sub-Objective 2: Synthesize bio-functionalized silicon that is capable of repelling and manipulating cells composed of stable and unstable phases of silicon and silicon oxides. 
Task 1: Bio-functionalization of silicon without surface damage via ultrashort pulse laser synthesis.

Task 2: Theoretical analysis of ultrashort pulsed laser assisted formation mechanism of biofunctionalized repelling silicon. Explore effect of peak power that is dependent on pulse duration as well as pulse to pulse separation time on the formation of bio-functionalized cell repelling silicon.

Task 3: Material characterization of bio-functionalized repelling silicon.

SEM, EDX and TEM: Study morphology of bio-functionalized silicon. Explore changes in substrate such as oxygen content.

AFM: Qualitatively analyze altered phases on bio-functionalized silicon.

Micro-Raman, X-ray photoelectron spectroscopy (XPS), X-ray diffraction (XRD): Quantitatively analyze the phases of silicon and silicon oxides on the surface of biofunctionalized silicon.

Task 4: Interaction of mammalian (fibroblasts) and cancer cells (HeLa) with bio-functionalized silicon.

> SEM: Study the adhesion mechanism and proliferation mechanism on bio-functionalized silicon.

Fluorescence microscopy: Selectively study actin cytoskeleton and nuclei of cells to determine proliferation, migration and adhesion mechanisms of cells to biofunctionalized silicon.

Sub-Objective 3: Synthesis of nanostructured silicon with modified phases of silicon and silicon oxides in addition to varying orientations of silicon and its potential application in cancer therapy.

Task 1: Fabrication of laser synthesized multi-phase multi-functionalized nanostructured silicon. Task 2: Theoretical analysis of formation mechanism and effect of peak power on phase and crystal orientation of nanostructures of silicon.

Task 3: Characterization of silicon nanostructures

SEM, EDX and TEM: Study morphology of multi-functionalized silicon nanostructures. 
Micro-Raman, X-ray photoelectron spectroscopy (XPS), X-ray diffraction (XRD): Quantitatively analyze the phases of silicon and silicon oxides on nanostructured silicon.

Task 4: Interaction of cancer cells (HeLa) with nanostructured silicon.

SEM: Study the adhesion mechanism and proliferation mechanism on nanostructured silicon. Explore formation, size and shape of lamellipodia and filopodia in HeLa cells to predict growth mechanism.

$>$ Fluorescence microscopy: Image and study actin cytoskeleton and nuclei of cells to determine proliferation, migration and adhesion mechanisms of cells on nanostructured silicon.

Task 5: Study influence of addition of gold on cancer cell control

$>$ Synthesize gold-silicon nanohybrid nanostructures via ultrafast pulsed laser synthesis

$>$ Characterize new phases of gold-silicon on nanostructures

$>$ Study interaction of HeLa cells with the nanostructures.

\subsection{Organization of the dissertation}

Chapter2 describes the design and synthesis of a novel three dimensional nanofibrous matrix that performs as a proliferative substrate for mammalian cells such as mouse embryonic fibroblasts.

Chapter 3 describes the nanofibrous structure in detail with studies on its biodegradability. Further, osteoblasts proliferation was measured and compared to fibroblasts.

Chapter 4 deals with the design and fabrication of a gold-silicon hybrid nanostructure that can selectively induce proliferation based on the concentration of gold.

Chapter 5 delineates the synthesis of sub-surface modified silicon with no surface damage. The growth and interaction of fibroblasts showed precise control of direction of cells. Further, this chapter presents a unique cell repelling property.

Chapter 6 portrays the precise control of proliferation and growth of cervical cancer cells. Cancer cell repelling and trapping is also discussed. 
Chapter 7 depicts nanostructured 'anti-cancer' silicon that induces controlled apoptosis in cervical cancer cells. A 95\% reduction in cancer cells is observed compared to control virgin silicon.

Chapter 8 elucidates an immiscible hybrid nanostructured gold-silicon core shell that prevents the growth and proliferation of cancer via apoptosis.

Chapter 9 summarizes the main results and proposes future direction for this research. 


\section{Chapter 2}

\section{Proposed novel method to mimic natural extra cellular matrix with advantages over previously generated matrices}

Nanostructured silicon has proven to be a promising candidate in tissue engineering. However, recent research on fabrication of silicon scaffolds has been limited to expensive, complex and time consuming lithographic techniques which require the addition of caustic chemicals. Moreover, these techniques generate structures which do not truly mimic the extra cellular matrix (ECM). In this chapter, a novel, interlinked, silicon nano-network fabricated by $\mathrm{MHz}$ ultrafast laser synthesis is introduced. Ultrafast laser synthesis is simple, rapid, free of any chemical additions, and can be carried out at ambient conditions. Variation in laser parameters resulted in alteration in the pore size and density of the silicon fibrous network. Microscopic analysis revealed a highly charged silicon network with elevated adhesion forces. In vitro bioactivity tests indicate the precipitation of bone like apatite in just three days. Cell proliferation studies on the silicon nano-network present a $300 \%$ increase in comparison to its bulk counterpart. Scanning electron microscopy analysis show healthy migration and attachment of cells on the silicon nano-network. This points to a correlation between elevated cell proliferation and the ECM-like structure of the silicon nano-network. This ECM-like silicon nano-network suggests significant potential not only in tissue engineering and regeneration but in other biomedical applications like biosensor detection as well.

\subsection{Introduction}

The synergy of materials engineering and cell biology has resulted in the development of novel biomaterials that could perform as a tissue scaffold. A tissue scaffold is required to provide a 3 dimensional environment for cells to grow and ultimately form a tissue. An ideal scaffold would mimic the extra cellular matrix (ECM) that is created by the cells of each tissue in the body [42]. In detail, an ideal scaffold should possess the characteristics of bioactivity, three dimensionality, promotion of cell adhesion and biocompatibility [43]. Additionally, cells in their natural environment are surrounded by nanoscale pores, fibers and crystals. Nanostructured metals, polymers, alloys and ceramics have all been well researched as potential scaffold materials [5], 
[44-47]. Nanostructured silicon, owing to its biocompatible and biodegradable nature, has attracted much recent attention. However, unlike other aforementioned materials, only very limited research work has been carried out to investigate the potential of nanostructured silicon as a scaffold material. This may be attributed to the fact that previously nanostructured silicon demonstrated relatively low bioactivity compared to other nanostructured materials. Bioactivity is a vital characteristic for an ideal scaffold. Bioactive materials form a bond between the tissue and the implant material or osseointegration which includes the nucleation of hydroxyapatite. If the bonding interface is not established, a fibrous tissue is formed around the implant causing fibro-osseous integration. This results in failure of the scaffold and rejection from the surrounding tissues. There has been some work done to study bioactivity on silicon. For instance, mesoporous silicon showed precipitation of calcium phosphate after a period of two weeks, whereas a glass-ceramic scaffold showed precipitation in just less than one week[48, 49]. Bioactive glass with high silicon content was studied as an alternative to overcome this drawback. However, bioactive glasses are very expensive.

Thus far, micro/nano silicon particles, porous silicon micro/nanostructures and silicon nanowires have been investigated for the purpose of a tissue scaffold. Coffer et al showed the precipitation of calcium phosphate on mesoporous silicon [48]. This early work proves that even though bulk silicon is bio-inert, mesoporous silicon demonstrates bioactivity. Further, Canham showed that it took seven days to see bioactivity in microporous silicon [50]. With nanostructures it is expected to see increased bioactivity since nano scaling would enable higher bioactivity. Bioactive substrates should also promote cell growth and proliferation. Recent studies have shown cell proliferation on different silicon micro/nano/meso structures. A case in point, Sun et al. showed that cell proliferation increases on silicon substrates with micropores when compared to meso and nanopores[51]. Agarwal et al. deposited a nanoporous silicon thin film of $55 \mathrm{~nm}$ thickness on silicon wafer which induced significantly higher cell proliferation than the conventional cell growth substrates and monolayer membranes to study cell-cell communication [52]. Fabrication of silicon protrusions was studied as a possible scaffolding candidate by Ranella et al. where micrometer spikes were created with nano protrusions [31]. However, research has shown that such protrusions penetrate the cells and prevent them from forming focal adhesions [53]. Sputter coating microstructures of metals with silicon substituted hydroxyapatite containing varying quantities of silicon, was researched for the purpose of fabricating a scaffold [54]. Here, 
increasing content of silicon showed better cell proliferation with mature cytoskeletons. This research cemented the fact that silicon is in fact a viable scaffolding material. Another study done by Kim et al. demonstrated that silicon nanowires of diameter $90 \mathrm{~nm}$ and length of $6 \mu \mathrm{m}$, grown on a silicon substrate were able to sustain cells for several days [55]. In all these works, silicon is fabricated into individual 1D or 2D nanostructures that are assembled in certain patterns. Even though none of them resemble the ECM in terms of structure and morphology, interlinking and random assembly of nanostructures, these silicon nanostructures demonstrated a marked increase in cell growth and proliferation [56-58]. There is a dearth of studies that have developed or examined the effect of ECM like nanostructures of silicon on cell growth/proliferation and bioactivity.

This chapter illustrates the fabrication, characterization and cell interaction of a three dimensional nanofibrous network [59]. This fabrication is done in ambient atmospheric conditions and does not need the addition of any harmful material (i.e. catalyst). The interlinked nanofibrous structure of the nano-network makes them particularly suitable for cell attachment.

\subsection{Experimental Detail}

An undoped $<100>$ oriented silicon wafer (University Wafers, USA) with $500 \mu \mathrm{m}$ thickness was cut into $2 \times 2 \mathrm{~cm}$ squares. The squares were washed with deionized water and then left to dry. These substrates were irradiated using a diode-pumped, Yb-doped femtosecond laser system (Clark-MXR Inc. IM-PULSE Series Ultra short Pulse Laser, USA) at 26MHz with laser material interaction times (dwell time) between 1 and $15 \mathrm{~ms}$ at a constant power of $15 \mathrm{~W}$.

The obtained silicon nano-network was characterized with a scanning electron microscope (Hitachi S 5200) and a transmission electron microscope (Hitachi H 700 CTEM). Analysis of pore size and particle size was carried out using ImageJ (available from the NIH website).

Measurement of zeta potentials of the nano-networks was carried out using a zetasizer (NanoZS90; Malvern Instruments, UK). The silicon nano-networks were mixed in $1 \mathrm{ml}$ of deionized water and transferred to a cuvette after which measurements were taken.

Force of adhesion of the nano-networks was measured using an AFM (NTEGRA, NT-MDT, Russia). A single crystal silicon tip (antimony doped) with a spring constant of $0.11 \mathrm{~N} / \mathrm{m}$ was 
used in contact mode. NT-MDT software was used to calculate the force of adhesion from the obtained graphs.

To test bioactivity, simulated body fluid (SBF) was made following the procedure detailed by Takadama et al., and kept at $4^{\circ} \mathrm{C}$ [60]. A calculated amount of SBF was heated to $36.5^{\circ} \mathrm{C}$ in a container into which the samples with nano-networks were deposited. To prevent gravitational effects, samples were placed perpendicular to the base of the container. The soaked samples were allowed to incubate for 3 and 10 days. The samples were then removed, dried and analyzed using SEM and EDX.

Cell-material interaction was tested with NIH 3 T3 mouse embryonic fibroblasts (ATCC, USA). Silicon substrates with nano-networks and control samples (plain silicon) were attached to 96 well plates using bio inert glue (Edmund Optics, USA). The cells were grown to confluence in tissue culture flasks and then seeded on to silicon substrates attached to 96 well plates at a density of $10^{5}$ cells $/ \mathrm{ml}$. The seeded silicon substrates were incubated for 48 hours. MTT assay was carried out to measure cell proliferation. Scanning electron microscopy was performed to assess attachment morphology of cells. After 48 hours of incubation, MTT assay and SEM analysis was performed to measure proliferation and attachment respectively.

For MTT assay, media was aspirated from the wells and $2.5 \mathrm{mg} / \mathrm{ml}$ of MTT (3-(4, 5Dimethylthiazol-2-yl)-2, 5-diphenyltetrazolium bromide, a yellow tetrazole) prepared in DPBS (Dulbecco's Phosphate-Buffered Saline) was added to each well. After another 4 hours of incubation, DMSO (Dimethyl Sulfoxide) was added to dissolve the insoluble formazan crystal [61]. Absorbance was measured using a UV-VISIBLE spectrophotometer (Shimadzu, Japan) at $540 \mathrm{~nm}$.

For SEM analysis, the cells were fixed using $2 \%$ glutaraldehyde and dehydrated in increasing concentrations of ethanol. After drying the samples at room temperature images were taken at varying magnifications using a scanning electron microscope (Hitachi SU1510).

\subsection{Results and Discussion}

\subsubsection{Characterization of silicon nano-network using SEM and TEM}

Figure 2-1 shows an SEM image of the silicon nano-network. The mechanism of nano-network formation is clearly explained in reference 16 [55]. Aggregation of the individual nanoparticles is 
more fusion than mere loose packing and hence the bonds between molecules are very strong [62], [63]. This aggregation leads to a fibrous 3-D nanostructure which is interlinked between layers. The surface charge of these nanoparticles is considerably high.Figure 2-1(c) also illustrates the formation of necks caused by fusion.

The SEM image depicting the nano-network shows that silicon nanoparticles aggregate to form chains, rings and bridges. These structures are interconnected, three-dimensional and porous. Furthermore, these structures are assembled randomly and display no particular order or pattern. The thickness, pore size and particle size of this nano-network can be varied using different laser parameters. A cross section of the nano-network shows that at the substrate-nano-network interface (base), the network is dense whereas the density at the top of the nano-network reduces. There was also an increase in pore size on the surface of the network with increasing thickness. The increasing deposition thickness of the nanoparticle-network compresses the pores at the base resulting in a more densely packed network.

\subsubsection{Interaction measurements using Zetasizer and AFM}

Zeta potential is an important physicochemical parameter as increase in zeta potential results in corresponding increase in cell proliferation [64]. Zeta potentials for the silicon nano-network are measured in relation to deposition thickness and the results are shown in Figure 2-2. The graph suggests a linear increase in negatively charged nano-network with increasing thickness. This can also be correlated to decreasing particle size. It has been previously shown that as particle size decreases the zeta potential increases [65]. A surface with negatively charged zeta potential is hydrophobic and adsorbs proteins on to its surface. This in turn leads to higher cell adhesion on the surface with higher zeta potentials [66]. A blank silicon wafer surface was tested as a control. In comparison, the wafers with deposited nano-networks, showed considerably higher zeta potential.

To further study interaction mechanism, force of attraction of the nano-network was measured using an AFM which gives an indication of the interactive forces of the nano-network. The AFM consists of a cantilever that interacts with the substrate. The cantilever is first brought into contact with the sample surface and is then retracted from the surface. 

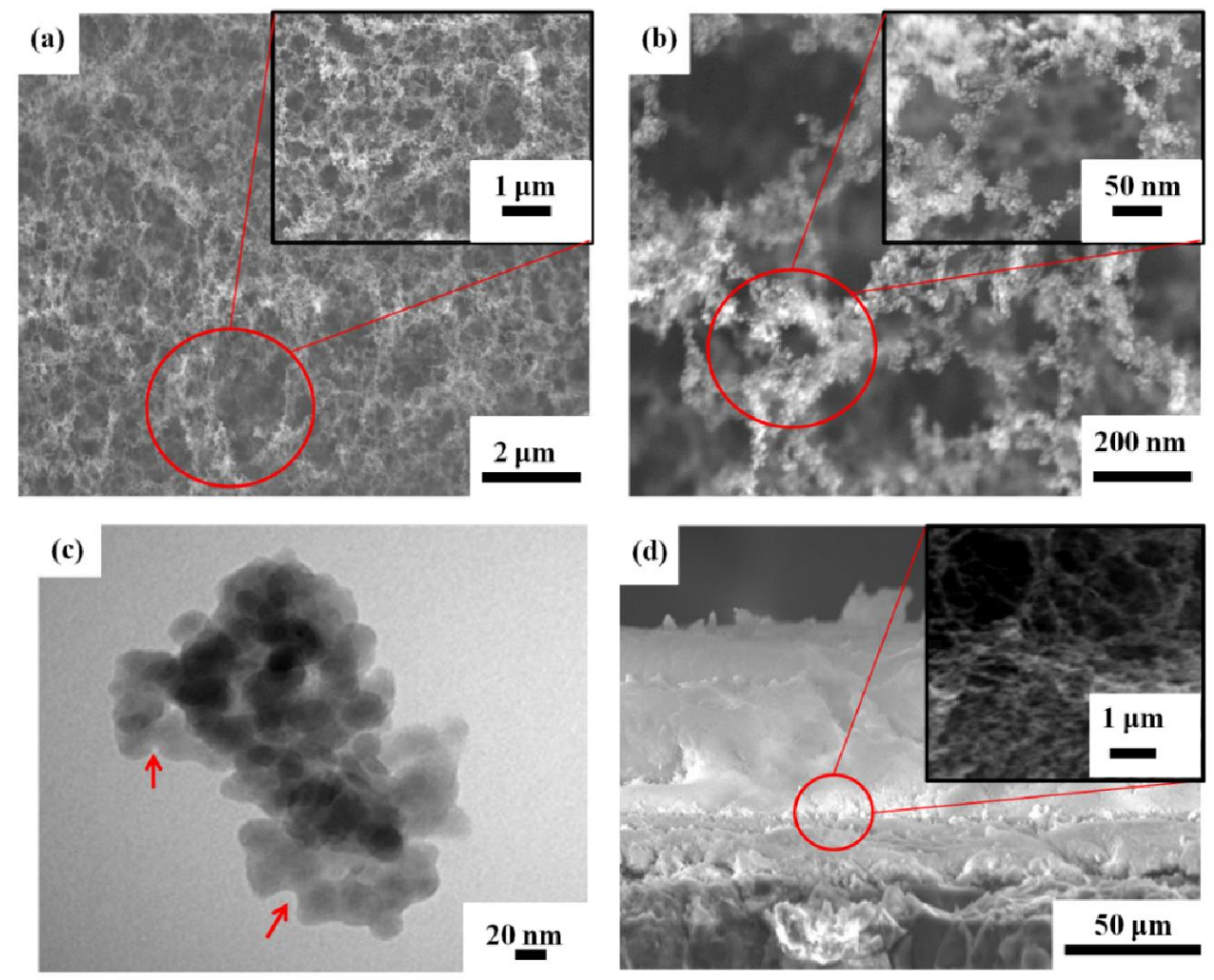

Figure 2-1: (a-b) SEM image of silicon nano network (c) TEM of silicon nanoparticles that combine to become silicon nano-network (d) Cross sectional SEM of silicon nano network showing decreasing density with increase in height.

The cantilever bends downwards towards the sample surface due to adhesion forces until a break point is achieved. At this point the cantilever retracts completely. Figure 2-3(b) shows an image of the force curve from the surface of silicon deposited with a nano-network. A blank silicon wafer was used as a control sample and its force curve is given in Figure 2-3(a). From the force curve the adhesion force can be calculated using $\mathrm{F}=\mathrm{k}^{*} \Delta \mathrm{l}$ where, $\mathrm{F}$ is the adhesion force $(\mathrm{nN}), \mathrm{k}$ is the spring constant $(0.11 \mathrm{~N} / \mathrm{m})$ of the cantilever and $\Delta 1$ is the deflection distance $(\mathrm{nm})$ [67]. The calculated forces are plotted in Figure 2-3Error! Reference source not found.(c). The adhesion forces increased dramatically with the deposition of a nanoparticle-network. With a thickness of $100 \mu \mathrm{m}$, the adhesion force is seven times higher than that on a plain silicon surface. The initial adherence of cells onto the nano-network depends on its surface characteristics. From the AFM 
interaction curves, greater forces of attraction were noticed for thicker nano-networks. Therefore, it is expected to see higher cell attachment on thicker substrates.

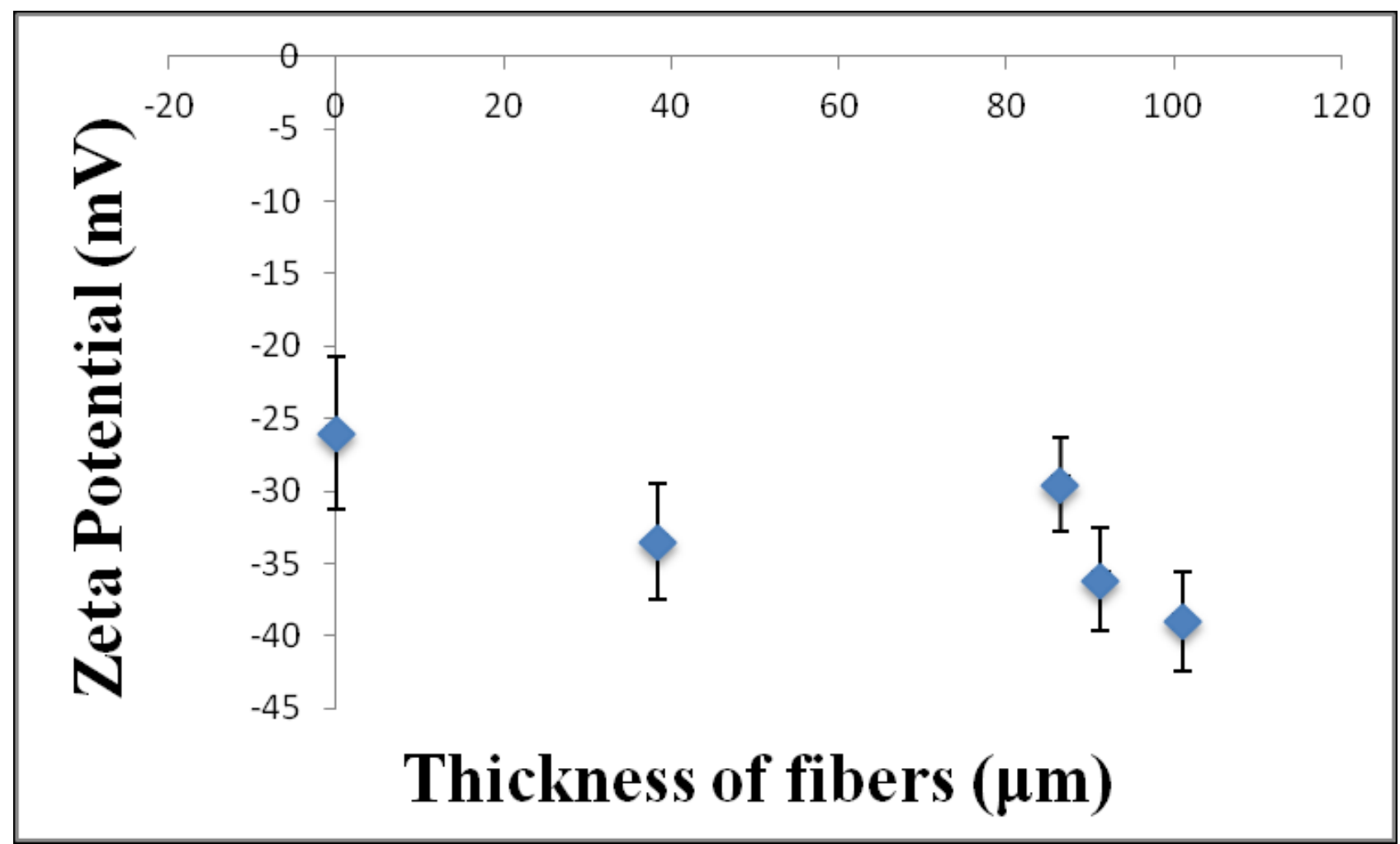

Figure 2-2: Zeta potentials Vs deposition thickness, $0 \mu \mathrm{m}$ thickness being the control sample.

\subsubsection{Bioactivity}

Poor bioactivity will result in fibrous tissue layer formation between the implant and the nearby tissues. This leads to rejection of the implant from the body. On the other hand, a bioactive material will enhance the formation of hydroxyapatite, promoting cell growth. Hydroxyapatite has a $\mathrm{Ca}$ : $\mathrm{P}$ ratio of 5:3. It has been shown that a fibrous micro/nano structure is effective in inducing bioactivity $[38,68]$. A simulated body fluid (SBF) test was done to study the bioactivity of the nano-network. Bulk silicon showed no apatite formation on its surface for a period of 14 days. Substrates with nano-networks were shown to have apatite formation after just three days of incubation. 

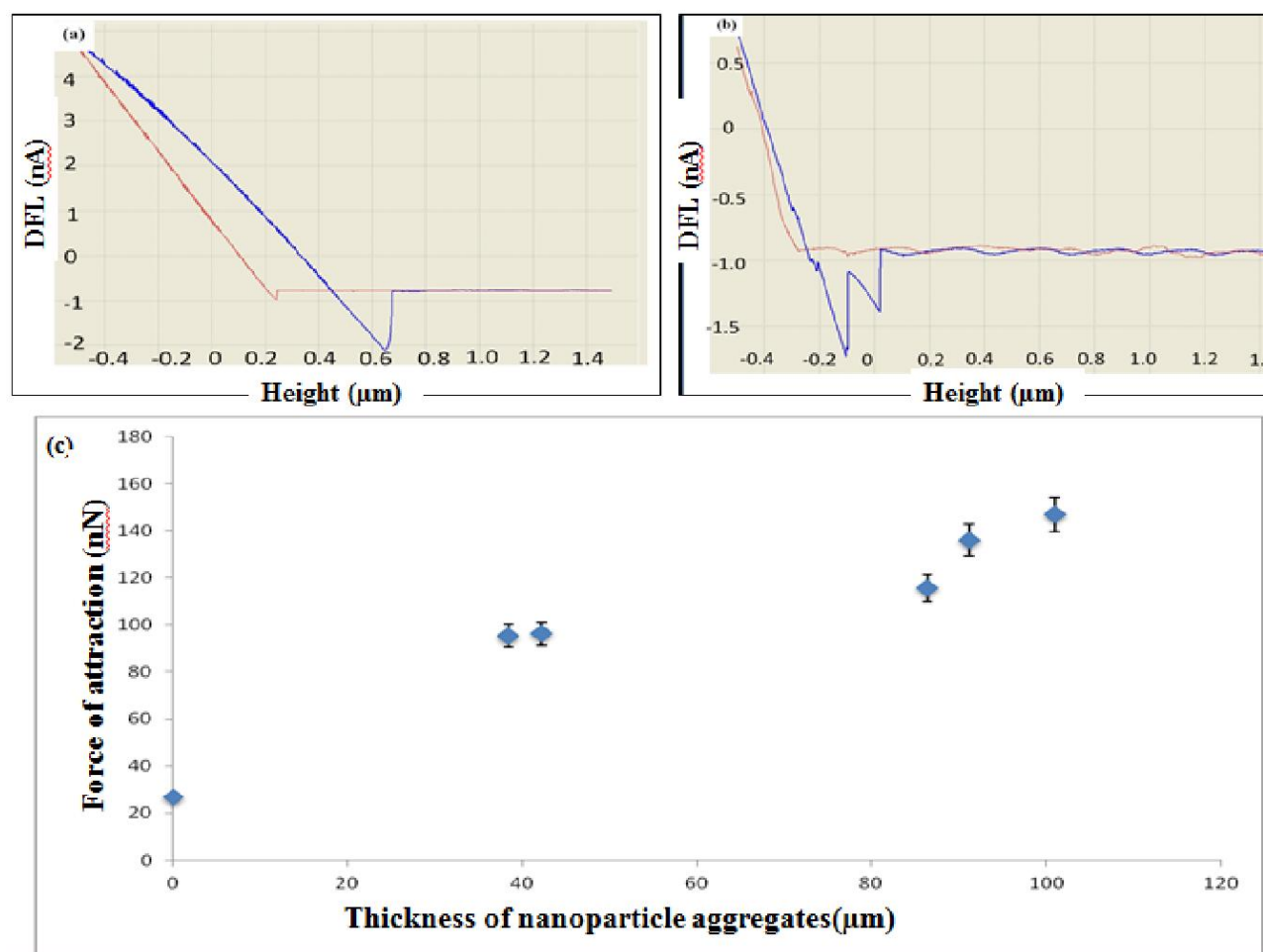

Figure 2-3: (a) force curve from a plain silicon surface (the red lines signify approach of tip towards the surface and blue lines signify retraction from surface) (b) force curve from nanonetwork deposition (c) Adhesion forces and deposition thickness. Data at 0 is control silicon.

The surface was covered with microspheres of 2-4 $\mu \mathrm{m}$ (Figure 2-4(a)). After 10 days, the entire surface was covered by apatite formation. Figure 5(a) and 5(b) shows the formation of hydroxyapatite on silicon nano-networks at both 3 and 10 days respectively. Figure 2-4(c-d) gives the EDX measurement used to compare the growth of apatite on silicon surfaces with and without a nano-network. The insets of Figure 2-4(c-d) indicate the location where measurements were carried out. The EDX analysis showed the presence of sodium, chlorine and magnesium in addition to calcium phosphate. The process of formation of calcium phosphate starts with silicon oxides forming Si-OH bonds on the surface from the water molecules present in the SBF. Si-OH has been shown to aid growth and nucleation of apatite. These molecules attract $\mathrm{Ca}^{2+}$ molecules forming calcium hydroxide bonds. Finally, this attracts phosphate ions forming hydroxyapatites. The structure and physicochemical structure of the nano-networks are responsible for increased 
bioactivity. Increased bioactivity indicates increased adsorption of proteins which in turn allows for the recruitment of more cells through attachment [69].
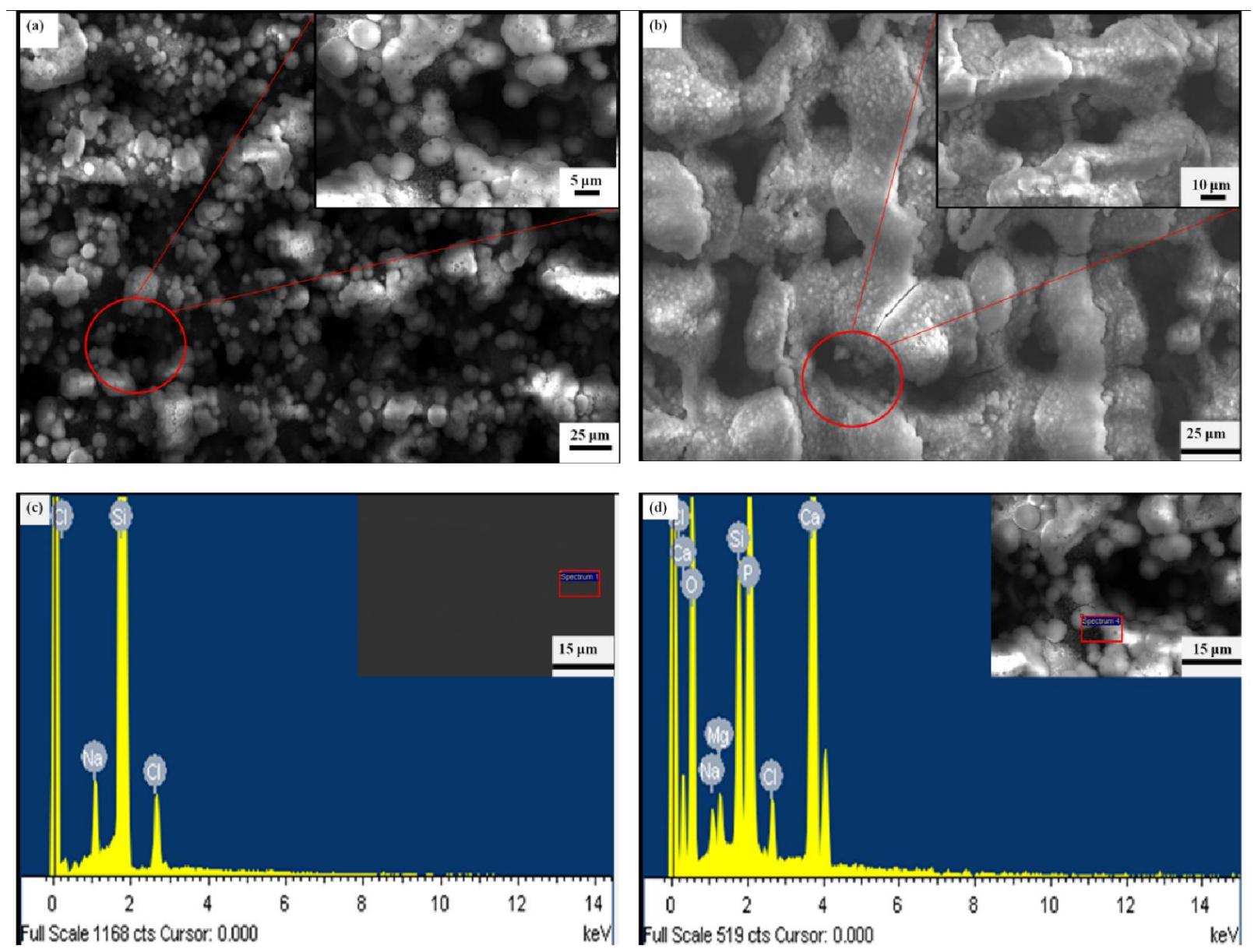

Figure 2-4: SEM micrographs of hydroxyapatite formation on silicon nano-network after (a) 3 days and (b) 10 days. EDS showing spectral analysis of silicon samples immersed in SBF for 3 days (c) virgin silicon (d) silicon with nano-network.

\subsubsection{Cell-biomaterial Interaction}

An ideal scaffold should aid in cell growth and differentiation apart from eliciting bioactivity. Interaction of NIH 3T3 mouse embryonic fibroblast cells on a silicon nano-network is reported. The relationship between increasing thickness of the nano-network deposition and cell proliferation was studied and the results are presented in Figure 2-5. To study the morphology and attachment of cells, SEM images were taken after 48 hours of incubation (Figure 2-6). The SEM images illustrate healthy proliferation of cells on the substrate. The cells showed multiple filipodia that extended across the nano-network. They formed strong adhesions which are 
evidenced in large pseudopodial projections. Ruffles on lamellepodia was also noticed. This indicated that cells were spreading well. The cells appear flattened and polarized indicative of migration. The increasing thickness significantly influenced cell proliferation. A t-Test was performed and a $p$ value of 0.002 was obtained showing significant increase in cell proliferation between control samples and silicon nano-networks. The obtained results reflected that cell proliferation may depend on pore size and/or particle size and on the ECM like structure. The fabricated nano-networks consist of nanoscale pores and nanoparticles. Li et al. noted that even though pore size is in the nano scale range, during amoeboid movement, cells have the ability to move fibers and manipulate them according to their needs [70]. Hence, for cells to manipulate fibers, flexibility of fibers is vital. During SEM and TEM analyses, it was noted that the silicon nano-networks at the top tended to be more flexible with higher porosity and bigger pore size. This implies that the structure of the nano-network with nanoscale pores plays a direct role in cell proliferation [71]. Nanoscale features organize the cytoskeleton and membrane receptors such that signaling between cells is improved. Protein adsorption is also said to improve with these nano features aiding integrin signaling [72]. Particle size was assumed to elicit differences in proliferation between differing thicknesses of nano-networks. However, further analysis revealed that the particles in the nano-networks with different thickness differed only in nano dimensions. And this small change in nano dimensions had no significant change in cell proliferation. Thus, the cell proliferation studies on the silicon nano-network conclusively proved that nanoscale dimensions have a positive effect on cell growth.

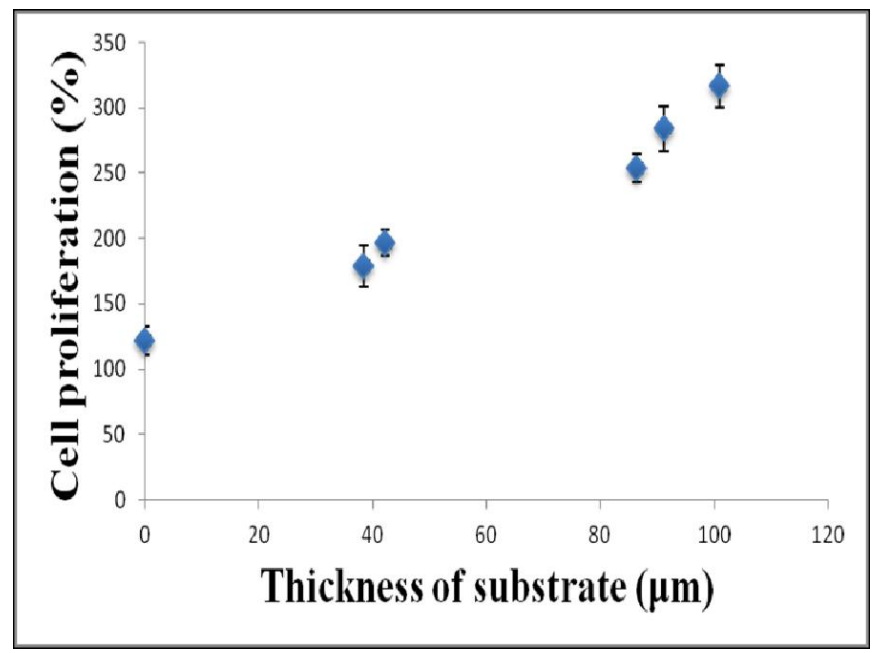


Figure 2-5: Increase in proliferation of NIH3T3 fibroblast cells as deposition thickness increases after 48 hours of incubation.
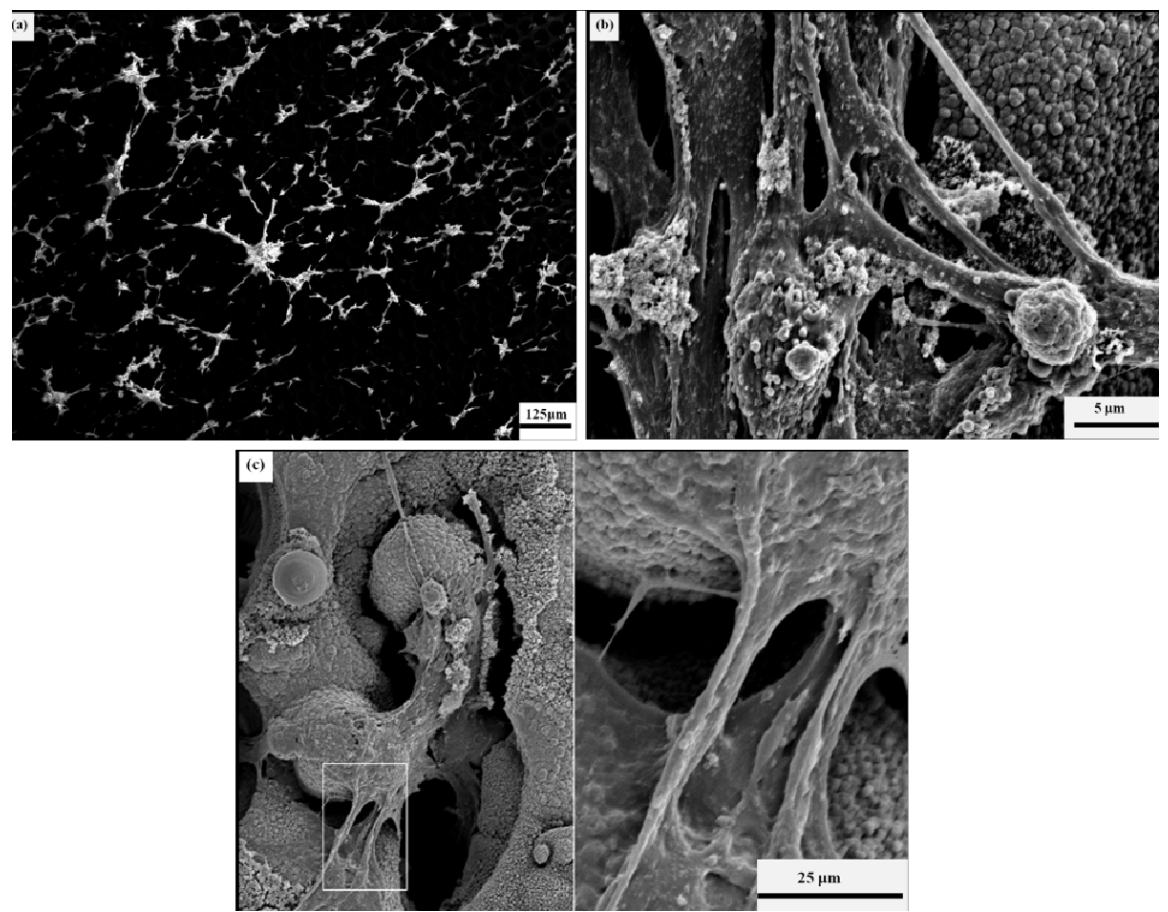

Figure 2-6: (a) SEM of fibroblasts on silicon nano-network formed at 10ms after 48 hours of incubation (b-c) Fibroblasts with multiple filopodia spreading out indicating strong adhesion and migration.

\subsection{Summary}

An ideal scaffold is sought after for its characteristics of biocompatibility, bioactivity and likeness to ECM. Expensive materials have been well researched at a nano level but a promising candidate, silicon was not well studied. In this chapter, a unique way to fabricate a novel interlinked nano-network whose structure and dimensions closely mimic the ECM is presented. This nano-network increased bioactivity, inducing calcium phosphate deposition in three days compared to bulk silicon where there was no precipitation after fourteen days. Results indicate that the nano structure as well as surface characteristics of this nano-network critically influenced bioactivity. The nanoscale structures presented cells with an ECM like environment. As a result, a $300 \%$ increase in cell proliferation is demonstrated. 1.6 times higher zeta potential 
and a sevenfold increase in adhesion forces were contributing factors to the increased cell growth and bioactivity. Nanopores, three dimensionality and bioactivity not present in previous silicon nanostructures make these nano-networks suitable replacements for current expensive scaffold biomaterials in the field of tissue engineering. 


\section{Chapter 3}

\section{Biodegradability of bioactive interlinked nanostructures and osteoblast interction studies}

This chapter presents a novel $\mathrm{MHz}$ laser synthesis method that permits sub-100nm scale structures on any material, including silicon that mimic the natural ECM. Owing to its three dimensional interlinked nature, the nanofibrous substrate is shown to guide the osteoblasts and fibroblasts to grow not only planarly to the surface, as is true for conventional scaffolds, but also expand and grow upward vertically. This method of synthesis demonstrates promise for novel three dimensional (3D) scaffolds that can assist in tissue and bone regeneration and a myriad of other applications such as drug delivery and biosensing.

\subsection{Introduction}

Tissue engineering has crossed the stage of nascence and is now emerging as a scientific discipline capable of generating feasible clinical applications to improve the quality of human life. The primary focus of tissue engineering is to create a three dimensional (3D) environment that can facilitate the growth and proliferation of cells [73]. The need for organ replacements to overcome the current shortage of organs is the chief concern. This is also a vital tool in generating in vitro models of organs to further investigate cell interactions and drug delivery. For bone regeneration, the site of injury needs progenitor cells to proliferate, migrate and adhere. These cells require a skeleton to guide their growth in a 3D fashion. Until now our understanding of cellular processes has been limited to two dimensional (2D) in vitro models. The cell morphology, its differentiation and proliferation and cell biomaterial interaction, all depend on the dimensionality of the substrate itself. It was noted that the interactions in 2D vary significantly from 3D [74]. Cukierman et al. showed that paxillin and $\alpha_{5}$ integrin co-localized as opposed to separately localizing and forming focal and fibrillar adhesions. They cultured human foreskin fibroblasts on 3D and 2D surfaces and found that 3D substrates were 6 times more efficient in forming specific cell-matrix adhesion structures than 2D surfaces [75]. This research reiterates the need for 3D in vitro models where physiological cellular responses can more precisely be predicted. 
There has been a plethora of research conducted to fabricate a 3D scaffold with favorable properties using micro/nano fabrication techniques. One of the earlier methods developed was particulate-leaching which was used to fabricate micro porous scaffolds [76, 77]. Although this method presented a simple way of processing porous substrates, they had certain limitations including the inability to vary pore shape and maintain the bond between pores. Particulate leaching produces periodically aligned pores where there is no communication between pores. With the arrival of computer assisted programming, 3D printing was a solution to controlling precise parameters of 3D scaffolds. This method also provides integrity to the structure and leverage with the design [78]. However, 3D printing also has certain limitations, one being the need for an optimum temperature, only certain materials like polymers can be employed, and nanoscale features cannot be achieved. Therefore, electrospinning materialized as a method of choice. Electrospinning employs an electric field that charges a polymer solution. After the solvent has evaporated, the fibers are collected on a metal screen [79]. Electrospinning, though widely used does not provide a 3D scaffold in the truest sense. It involves layer on layer of fibers but the cross linking between individual fibers is not robust and strong. Another limitation of electrospinning is its inability to generate nanoscaled features; only sub-micron features in the range of 200nm have been shown [80-83]. Furthermore, electrospinning has not been able to generate nanofibrous structures in varying shapes and only selected materials can be used. Owing to its abundance, biocompatibility and nontoxicity, silicon is being highly researched as a material of choice but cannot be employed in the electrospinning technique [84]. Electrospinning is limited in the materials that it can employ, silicon being one that cannot be used.

To negate these inherent drawbacks present in other techniques, a novel method to fabricate a three dimensional scaffold which will enable tissue and bone regeneration is presented. To the best of our knowledge, a three dimensional scaffold to this degree of likeness to the natural extra cellular matrix (ECM) has not been fabricated. The ECM should posses three dimensionality and nanoscale [72]. A MHz femtosecond laser is employed to engineer these nanostructures directly on silicon wafers. When the laser interacts with the surface of silicon, it results in the formation of gaseous species which condense to form silicon nanoparticles that aggregate to form a nanofibrous interlinked, randomly oriented, nanoporous, and 3D structures [59]. This method directly generates patterned silicon oxide nanofibrous structure in a single step. Silicon oxide has shown to be biocompatible for cells [85]. Silicon has also been proven to be an indispensable for 
bone growth [86-88]. This method is inexpensive, quick and our work has shown that any material can be used [40], [63], [89-91]. By varying laser parameters, it is seen that any shape and thickness of the nanofibrous structure can be achieved. A degradation study shows the silicon nanofibrous network has a very slow degradation rate. Mouse embryonic fibroblasts and mouse calvaria-derived pre-osteoblastic cells were used to study cell-biomaterial interaction in applications of tissue and bone regeneration.

\subsection{Experimental Detail}

\subsubsection{Characterization studies}

Scanning electron microscopy (Hitachi S 5200) (SEM) was employed to characterize the nanofibrous silicon networks. The individual nanoparticle size was studied using transmission electron microscopy (Hitachi H 700 CTEM) (TEM). To separate the nanoparticle networks from substrate, the samples were dipped in isopropanol solution and then ultrasonically vibrated. A drop from this solution was then placed on a copper mesh and then allowed to dry in a desiccator. To calculate pore size and particle size ImageJ was used. Energy-dispersive X-ray spectroscopy (EDS) is performed to study the material composition of these silicon nanofibrous networks.

\subsubsection{Degradation Studies}

To assess the rate of degradation, the silicon nanofibrous network was immersed in Dulbecco's Modified Eagle Medium and allowed to incubate at $37.5^{\circ} \mathrm{C}$ and $5 \% \mathrm{CO}_{2}$. Preceding their immersion, the substrate containing the silicon nanofibrous network is weighed. The silicon nanofibrous networks were assessed for degradation after 6, 24, 48 hours and 1 week. At the specified time periods, the samples were removed and dried under high pressure and high speed for one hour. The weights of each sample were measured again. All experiments were performed in triplicates and plain silicon wafers are used as control samples.

\subsubsection{Cell culture studies}

Cell- biomaterial interaction was studied using NIH 3T3 mouse embryonic fibroblasts and mouse calvaria-derived pre-osteoblastic cells (ATCC, USA). Samples were immersed in petri dishes with cells seeded at a density of $10^{5}$ cells $/ \mathrm{ml}$. All cell culture experiments were conducted in triplicates. After a day the samples were removed and the fixing process was initiated. The 
samples were fixed in $2 \%$ glutaraldehyde in $0.1 \mathrm{M}$ sodium cacodylate buffer $\mathrm{pH} 7.3$ for an hour. After immersing in $0.1 \mathrm{M}$ sodium cacodylate buffer with $0.2 \mathrm{M}$ sucrose $\mathrm{pH} 7.3$ for 20 minutes the samples were dehydrated in increasing concentrations of alcohol for 20 minutes each. The samples were then critical point dried and imaged using a scanning electron microscope.

\subsubsection{Statistics}

All experiments were performed in triplicates. Average and standard deviation were calculated from collective results.

\subsection{Results and discussion}

\subsubsection{Fabrication and characterization of a three dimensional silicon nanofibrous network}

$\mathrm{MHz}$ femtosecond laser irradiation of silicon results in the direct pattering of silicon nanofibrous networks on silicon. The volume and thickness of the nanofibrous structure are controlled with laser parameters such as dwell time and scanning speed. When the laser strikes the surface of silicon, a region of extreme heat is created. This causes vaporization and the materialization of a plasma plume. The plume expands outwards causing reduction in temperature and pressure. This results in condensation of the gaseous species causing nucleation. These nuclei, once they reach critical density, condense to form nanoparticles rather than micro particles[59, 92]. These nanoparticles then aggregate to form a nanofibrous network. The frequency of pulses is higher than the nanoparticle formation rate which provides a continuous inflow of vapor. By varying the fluence of the laser, different thickness with different shapes were obtained. Moreover, the region of irradiation can be controlled. For instance, in Figure 3-1(a-d), the shape of a man's face was irradiated. All facial features that are three dimensional- nose, ears, lips etc. are made of silicon nanofibrous network. The thickness, density, pore size and particle size of this nanofibrous network is also controlled by different laser parameters. EDS analysis shows the presence of silicon and oxygen in the silicon nanofibrous network (Figure 3-2). It has been shown that silicon oxidizes at $700-1300^{\circ} \mathrm{C}$ [93]. The high frequency of the laser pulses irradiating the surface of silicon causes it to oxidize as droplets and then agglomerate forming pores and nanoparticles of different sizes. The formation of silicon oxide is crucial as studies have shown that silicon oxide is favorable for cell growth [85]. 


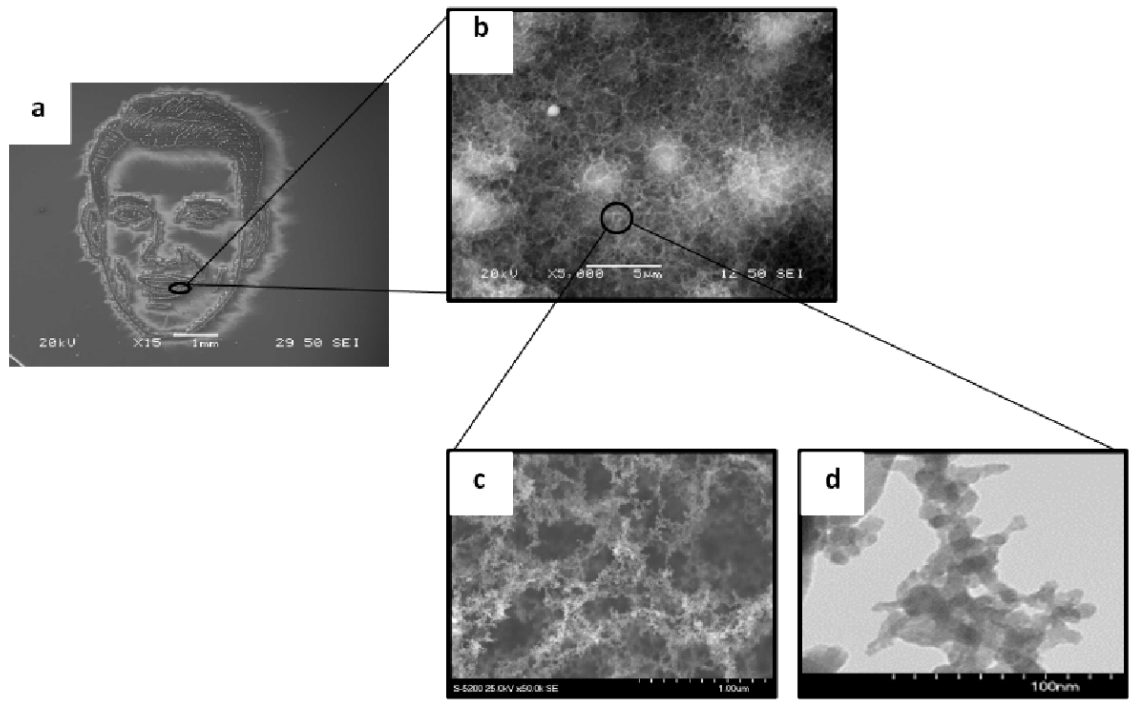

Figure 3-1: (a) Possible scaffold shapes that could be generated from $\mathrm{MHz}$ femtosecond laser synthesis (b) Required areas that have silicon nanofibrous network (c) Magnified image showing structure of the nanofibrous network by aggregation of nanoparticles (d) TEM of the nanofibrous network.
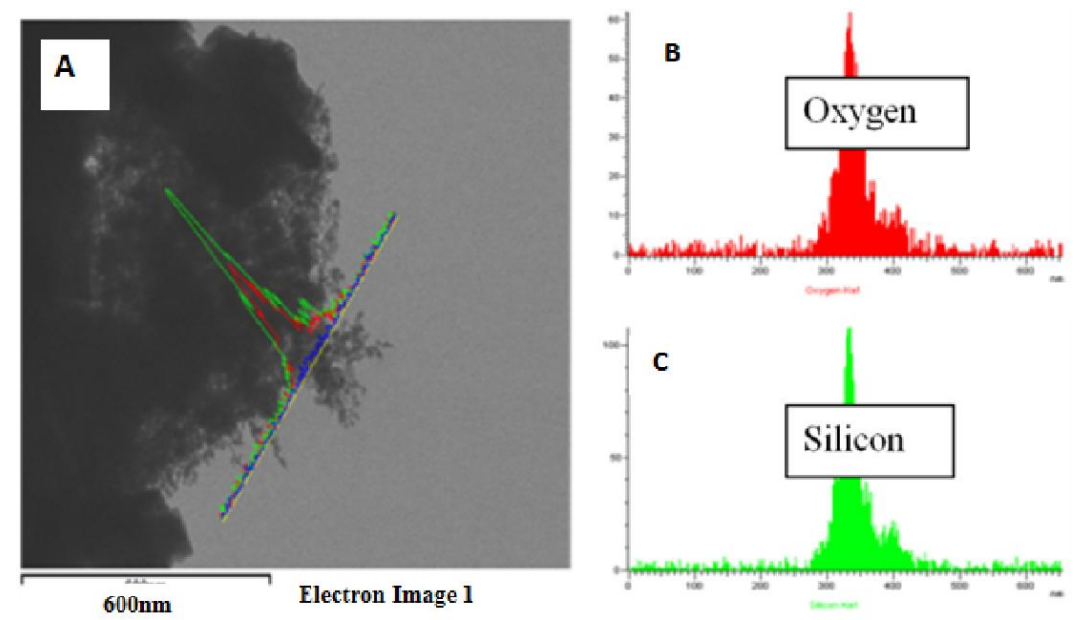

Figure 3-2: EDS analysis of the silicon nanofibrous network revealed silicon oxide nanoparticles.

(a) TEM of silicon nanofibrous network (b) Percentage of oxygen present (c) Percentage of silicon present. 
The pore size and particle size is shown to vary according to the dwell times. An increase in dwell time caused an increase in pore size and a decrease in particle size. When the dwell time is increased, the amount of heat being accumulated at the surface of silicon is very high. This results in more material being ejected from the surface. Due to such high temperatures, the viscosity of the molten material is reduced and the material becomes very mobile [37]. The phenomenon of self-absorption also comes in to play here. The laser light that is incident on the surface of silicon is also incident on already ejected material. This process of self-absorption breaks down the ejected gaseous material further, leading to a reduction in particle size. This consequently agglomerates to form a nanofibrous network [13]. This decrease in particle size subsequently increases the pore size. Therefore, a direct correlation between particle size, pore size and dwell time is perceived.

\subsubsection{Degradation Study}

An in vitro degradation study was carried out without the use of cells but with cellular media. The degradation rate of the scaffold was assessed over a period of 1 week. The rate at which the scaffold degrades plays an important role in cell regeneration. The degradation was monitored at 6,24 , and 48 hours, and after 1 week. Figure 3-3 presents the percentage of degradation over different time periods. A very small percentage of degradation was noticed over 1 week. The initial degradation is attributed to top layer of the silicon nanofibrous network. As shown in the inset Figure 3-3, the silicon nanofibrous network on top is very less dense compared to the lower part. The larger pore sizes that are visible are also contributing factors to degradation. After the top layer has degraded, the scaffold retains its integrity. This very small percentage of degradation is credited to the strong bonds between each nanoparticle, evident from the necks formed on silicon nanoparticles in TEM images (Figure 3-1(d)). With higher dwell time, the amount of energy being targeted at silicon is significantly higher. The amount of gaseous species being expelled into the plume is higher. The agglomeration of higher number of species results in higher density. But as we move away from the base of the substrate, the density of nanofibrous network decreases owing to fewer gaseous species present in the plume. 


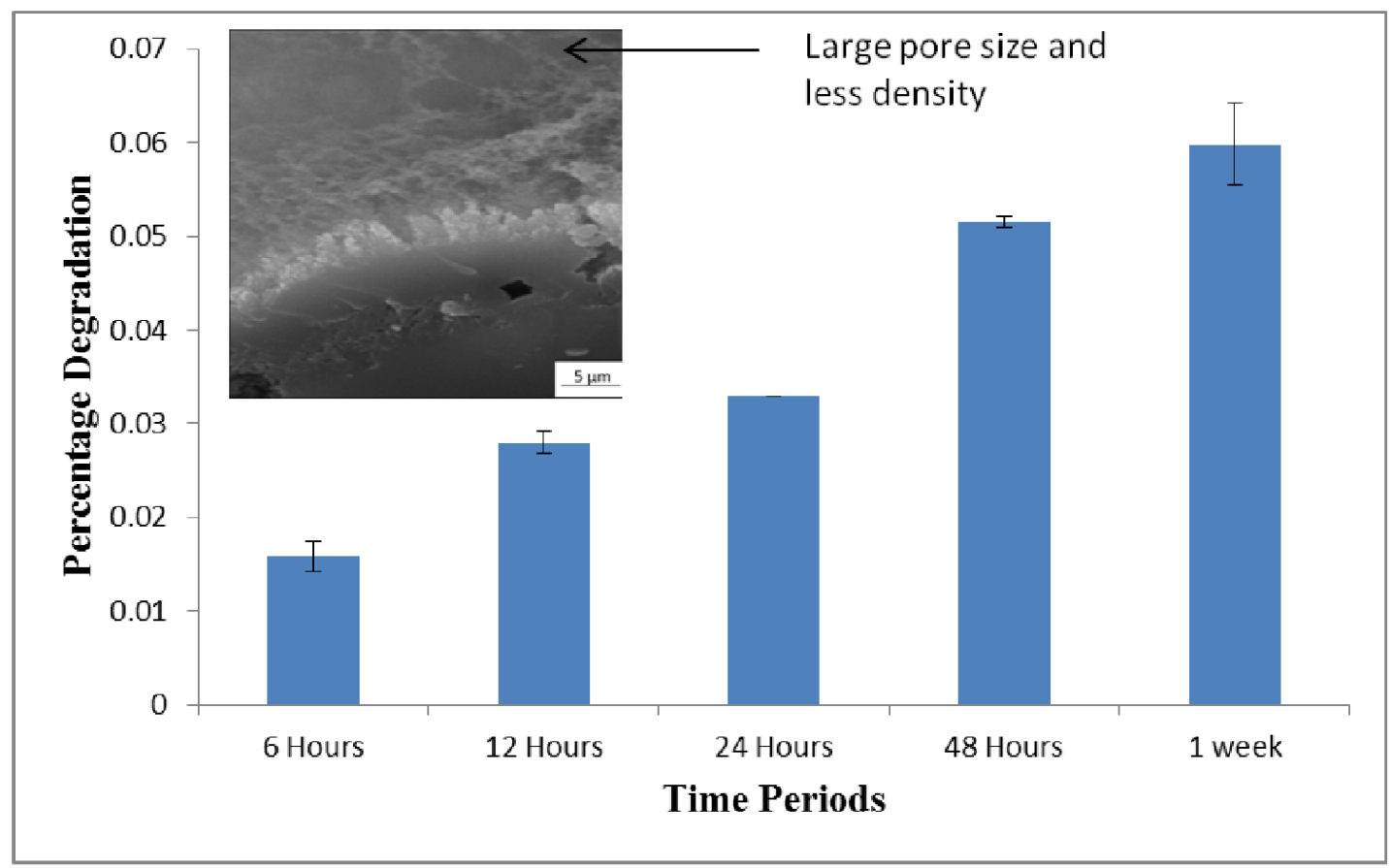

Figure 3-3: The degradation rate of the silicon nanofibrous network against different time periods is plotted. The inset represents a cross section of the silicon nanofibrous network that contributes to the rate of degradation. It shows decreasing density and decreasing pore size up to down.

The strength of this silicon nanofibrous network is vital in tissue regeneration. Although it is of prime importance that the scaffold be biodegradable, the rate at which it degrades is critical. The scaffold should sustain its integrity and provide a skeleton to facilitate guided growth to the cells.From the data obtained; an extrapolation of the findings to assert the scaffold's integrity until the cells have created their own matrix is made. Once the cells have proliferated and developed well, extra cellular matrix is produced by the cells providing a matrix for it grow further.

\subsubsection{Cell growth and analysis}

To assess the biocompatibility and efficiency of the silicon nanofibrous network, cell-biomaterial interaction was studied. NIH 3T3 mouse embryonic fibroblasts and MC3T3- E1 mouse calvaria- 
derived pre-osteoblastic cells were seeded on silicon nanofibrous networks. The interaction was assessed using scanning electron microscopy. It was noticed that the cells began to spread in the first few hours of seeding. Figure 4 shows the growth of fibroblasts on the silicon nanofibrous network. Figure 3-4 (a) shows the growth of a cell perpendicular to the substrate. This guided growth is facilitated by the nanofibrous silicon network. The interlinking between the layers of nanofibrous growth enables perpendicular growth. This is typical of growth patterns in the natural ECM, but has seldom been observed on in vitro scaffolds. Figure 3-4(b) shows the formation of a cluster of cells that are growing in all dimensions, in accordance with the 3D structure of the silicon nanofibrous network. This corresponds to an area with thicker silicon nanofibrous network. The pore sizes are smaller than the diameter of the cells and the fibers are flexible to permit penetration. The height of this layer-on-layer silicon nanofibrous network was calculated from SEM cross sections. Figure 3-4(c) shows the growth of cells on all layers on this nanofibrous structure. The cells were able to penetrate and grow on all layers. The cross linking between the layers provides guidance. After conducting SEM examinations of cross sections of this nanofibrous networks, the height of these nanofibrous networks was calculated. The average height was $100 \mu \mathrm{m}-150 \mu \mathrm{m}[94]$.
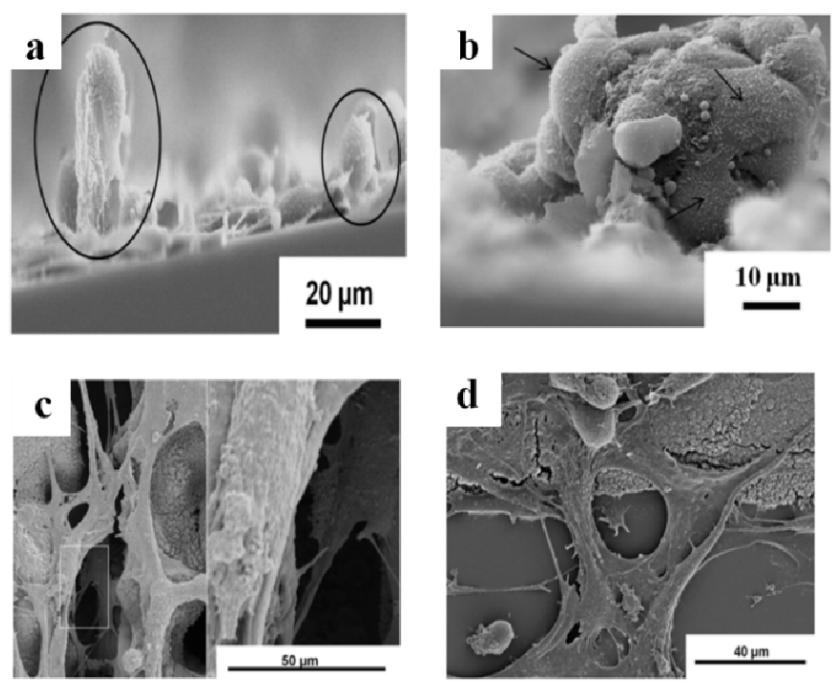

Figure 3-4: Various shapes of cells produced after 24 hours of seeding. (a) The circles indicate cells that have grown perpendicular to the surface (b) Multiple cells growing three dimensionally (c) Cells growing at different depths (d) Cells able to traverse across heights of $>100 \mu \mathrm{m}$ migrate and proliferate. 

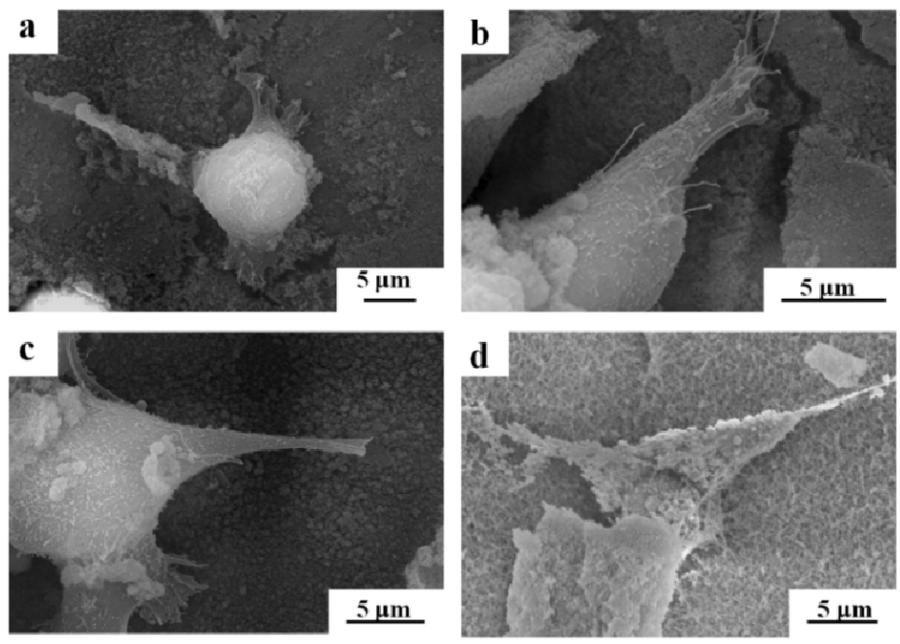

Figure 3-5: (a) A fibroblast that is rounding to divide. (b) The filopodia of the fibroblast interacting with the nanofibrous network. (c) The lamellae extending to adhere to a different area. (d) A fibroblast spreading out to adhere and grow on the silicon nanofibrous substrate
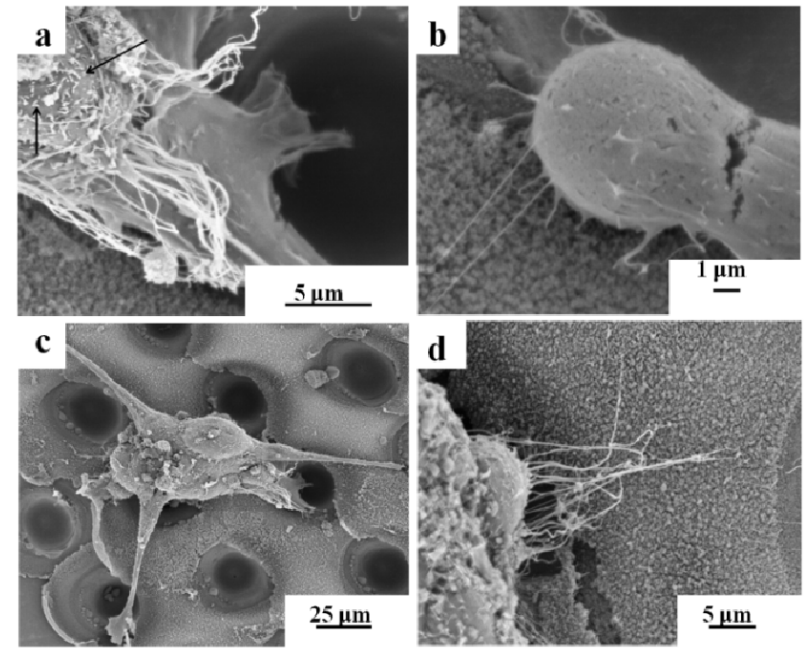

Figure 3-6: (a) Arrows pointing out the microvilli on osteoblasts (b) Lamellae of the osteoblasts extending with filopodia further extending out (c) A clump of osteoblasts, where the thickness of silicon nanofibrous was higher (d) Filopodia of the osteoblasts interacting with the biomaterial surface.

Figure 3-4(d) illustrates a cell that is able to grow by climbing over the nanofibrous network. Therefore from these images it is seen that cells are able to grow perpendicular to the base, in 
clusters and are able to traverse different heights. A closer look at the individual cells, both fibroblasts and osteoblasts revealed the interaction between the cells and the silicon nanofibrous network. Generally all the cells were flat and polarized. This is an indication of good adherence between the cells and the biomaterial [95]. A small percentage of cells were seen rounding up before cell division. Figure 3-5 shows cells observed in a small area, each of them at different stages of growth. Figure 3-5 (a) shows a fibroblast rounding up before cell division. Around this cell all the other cells were flat and polarized showing different stages of growth of the cells in a small area. Figure 3-5(b-c) shows the extension of lamella on to the surface of the silicon nanofibrous network. These lamellae also show ruffles. This indicates spreading and movement of the fibroblasts [95]. Figure 4(h) shows a cell that is flat and polarized. This is indicative of good health of the fibroblasts [96]. Osteoblasts showed similar characteristics with the presence of more filopodia and extended cell growth. Figure 3-6(a) showed the presence of cell processes called microvilli on the surface of the cell, indicated by arrows. The presence of microvilli has been linked to better cell adhesion and migration [97]. Figure 3-6(b) shows the filopodia that has attached itself to nanoparticles of the silicon nanofibrous network. It also reveals that the osteoblast is in the process of migration. The cell is moving in a presumed direction and has left a small part of its cytoplasm. Figure 3-6(c) shows a clump of osteoblasts that have clustered together owing to the increased thickness of the silicon nanofibrous network. Figure 4(1) shows the interaction of multiple filopodia with the silicon nanofibrous networks. A comparison with other similar research studies showed that our silicon nanofibrous network was able to elicit more filopodia and lamellae and other such healthy cell processes in the cells. This showed higher adhesivity and healthier growth pattern in our cells cultured on the silicon nanofibrous network [98, 99].

There were some notable differences between the growth and attachment patterns of the fibroblasts and osteoblasts. The fibroblasts and osteoblasts showed good adhesion to the nanofibrous silicon network. The sizes of the osteoblasts were bigger compared to the fibroblasts. The osteoblasts showed higher number of filopodia compared to the fibroblasts for the same period of time. At further magnification, it was noticed that the filopodia of both cells attached to singular nanoparticles in the silicon nanofibrous network. Each of these singular nanoparticles makes for adhesion points for the cells. Furthermore, these singular nanoparticles make up the three dimensional structure for guided cellular growth. 
The primary objective of a 3D nanofibrous network is to mimic the natural extra cellular matrix. The same has been attempted previously with various methods. For example, Zhang et.al showed the biocompatibility of collagen coated poly ( $\varepsilon$-caprolactone) nanofibers[99]. Although in nano dimensions, a truly three dimensional network with interconnected layers was not fabricated. These electrospun scaffolds are just layer-on-layer of nanodimensional material compressed together. Our 3D silicon nanofibrous network is well interconnected between layers while being nanodimensional. Hence, a natural ECM like environment is elicited. When cells are forced to grow on 2D surfaces, they attempt to adapt to false and rigid environments. This results in imprecise outcomes [58]. Not only a three dimensional structure, but the ECM also requires nanoscaled structures to promote cell adhesion and growth. With a nanoscaled scaffold, due to higher surface area, the cells are able to interact with a considerably higher number of receptors (Figure 3-7). This is one of the main advantages of our structure, as other methods produce only smooth fibers. The cells have been known to prefer fibers with diameters smaller than its diameter [100]. It has also been proved that conformation of adsorbed proteins is higher on nanoscaled surfaces. Due to higher surface receptor and ligand interaction, the cells are able to proliferate and migrate better. Figure 3-8(a) shows the effect of nanoparticles size on cell proliferation. It is seen that the cells preferred nanoparticles that were in the range of $6-8 \mathrm{~nm}$. As the nanoparticle size increased, the percentage of cell proliferation decreased. This trend may be attributed to the fact that fibroblasts prefer nano surfaces [101]. Pore size also plays a significant role in the proliferation of cells. Figure 3-7(b) shows the increase in cell proliferation with increase in pore size. The pore sizes on this silicon nanofibrous network permitted the formation of integrin clusters at a distance that the cells preferred [102]. Additionally, since the fibers at the top of the silicon nanofibrous network is highly flexible, the cells are able to manipulate the fibers according to their needs. Further, an increase in pore size allows for better gas diffusion, nutrient supply and waste disposal [103]. 

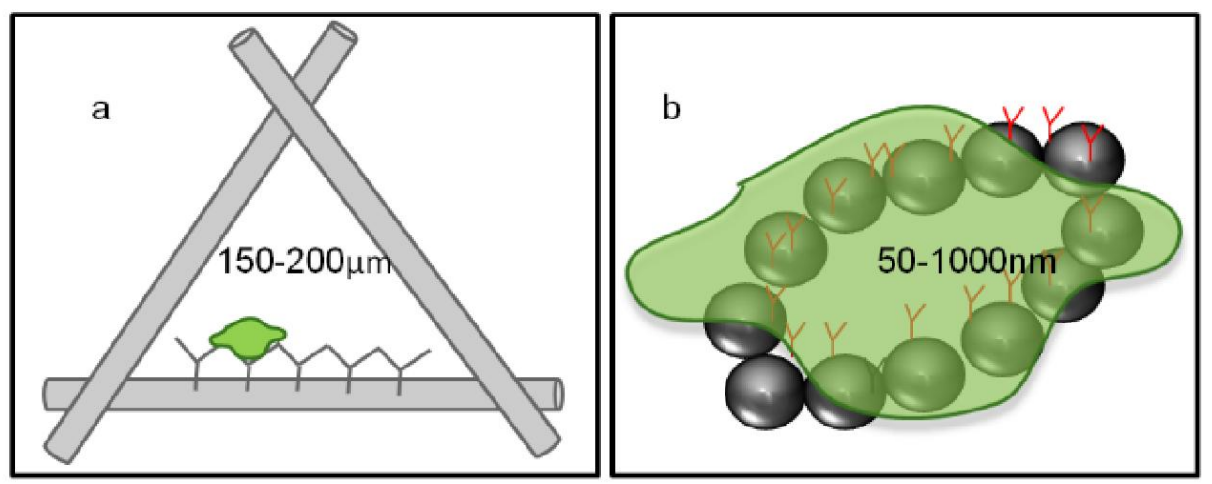

Figure 3-7: Illustration of cell-biomaterial interaction of microscale (a) compared to nanoscale (b). Nanoscale dimensions show superlative interaction
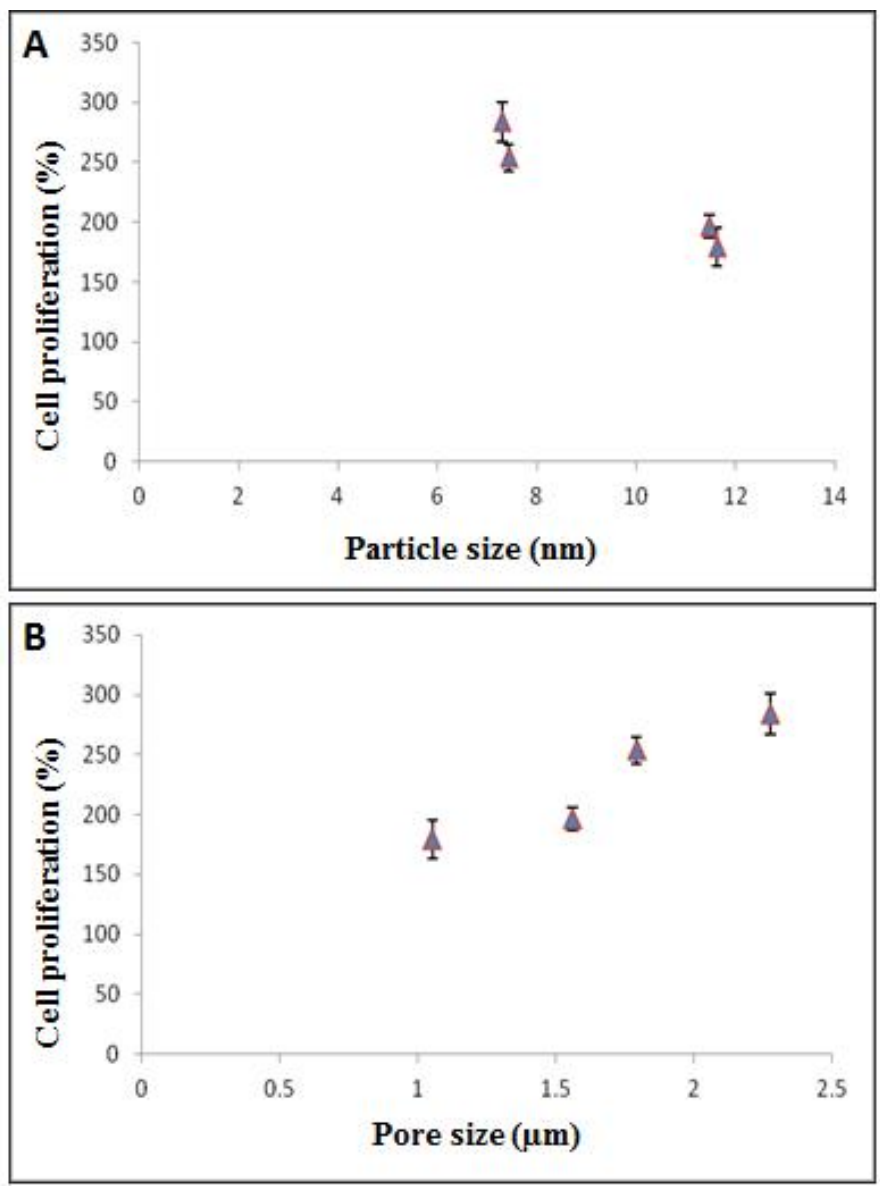

Figure 3-8: (a) Effect of particle size on cell proliferation after 24 hours of incubation and (b) Effect of pore size on cell proliferation after 24 hours of incubation. 


\subsection{Summary}

A novel technique to directly pattern a three dimensional silicon nanofibrous network in a single step is proposed. A three dimensional ECM-like scaffold has not been achieved before. This method provides an alternative to the currently widely used but inefficient technique of electrospinning to generate ECM-like nanofibrous structures. Unlike electrospinning, this method is able to generate a nanoporus, randomly oriented silicon nanofibrous network. The mode of generation is ascribed to the process of nucleation and condensation. This is the only method that is able to synthesize nanofibrous networks of any shape and thickness. This is achieved by controlling various laser parameters. This enables selective growth of cells in areas of requirement. An ideal scaffold should be biodegradable and degradation on studies on this silicon nanofibrous network revealed substantial strength and slow rate of degradation. Cellbiomaterial interaction studies showed good cell growth, migration and proliferation. The superlative growth of the cells is attributed to the morphology and topology of the silicon nanofibrous network. The nanoscale dimensions, the three dimensional nature, the pore and particle size are all thought to have played a role in proliferation and growth. This silicon nanofibrous structure has demonstrated the potential to be used as a material of choice for tissue regeneration. 


\section{Chapter 4}

\section{Optimization of the functionalization of bioactive three dimensional nanofibrous network with the addition of gold as an enhancer}

To enhance the functionalization of the three dimensional nanofibrous network, the addition of gold is explored. In this chapter, a nanofibrous hybrid of gold and silicon fabricated by femtosecond laser synthesis for tissue engineering applications is presented. The formation of a completely new phase, $\mathrm{Au}_{3} \mathrm{Si}$ (212) is reported. The formation mechanism is explained by vapor condensation. Particle sizes of 2-10 nm and 37-49 nm for gold and gold concentrations of 35$78 \%$ are achieved. The effect of this hybrid on cell growth was assessed using fibroblasts and osteoblasts. There was a significant decrease in both osteoblast and fibroblast proliferation with the increase of gold in the hybrid nanostructure. This novel hybrid nanofibrous matrix provides a method to effectively control the proliferation and adhesion of cells. Femtosecond laser synthesis presents a new standard by which not only a single element biomaterial but also multiple immiscible element hybrid biomaterials can be fabricated. This technique provides a paradigm shift in the fabrication of novel nanostructured immiscible hybrid biomaterials.

\subsection{Introduction}

The desire for new functions has generated an insatiable demand to develop novel functional nanostructured biomaterials. Most biomaterials are products of single elements, like metals, polymers and ceramics. This pure composition cannot satisfy all the current needs [9], [40], [69], [96], [104-107]. It was perceived that mixtures of two or more elements display superior properties when compared to their single counterparts. For instance, silk based scaffolds demonstrated excellent mechanical properties but did not provide a 3-D environment for cell growth. Therefore, Chen et al. introduced self-assembling peptide nanofibers to the silk scaffolds to enhance its three dimensionality [108]. Similarly, Lei et al. added silica to pure gelatin scaffolds to enhance the biodegradation stability, bioactivity and mechanical properties [109]. 
These research studies demonstrate the advantages of hybrid biomaterials that combine two or more elements.

Current fabrication mechanisms deal with bulk or macro/micro hybrids. Particularly, in the case of metals, fabrication mechanisms are limited to sputtering and chemical methods. Gold, for instance, is a highly sought after element in hybrid scaffolds. Numerous studies have been conducted to study the effects of elemental gold nanoparticles on cells. Pan et al. showed that cell death is dependent on the size and concentration of the gold nanoparticles [110]. They found that cells were most sensitive to $1.4 \mathrm{~nm}$ gold nanoparticles. Contrary to their study, Shukla et al showed that concentrations under $100 \mu \mathrm{M}$ and sizes of $3-8 \mathrm{~nm}$ did not cause cytotoxicity in macrophages [111]. Another study showed that gold nanoparticles with sizes 4, 12 and $18 \mathrm{~nm}$ did not cause any hindrances in cellular functions [112]. Hence, the possibility of incorporating gold to hybrid biomaterials to selectively control cell growth was perceived. However owing to its strength and inertness, gold cannot easily form nanostructures with other materials.

Despite the fact that hybrid materials demonstrate superior properties, fabrication of nanostructured hybrid biomaterials has not been demonstrated. Currently, sputtering gold on previously assembled nanofibers is used to possibly mimic nanostructured hybrid materials. This technique is flawed owing to the significantly weak bonds formed between gold and the nanofibers. This leads to disassembly during cell culture. Other techniques have thus been sought to produce gold or gold composite nanofibers. For instance, Kim et al. generated gold poly (ethylene oxide) (PEO) nano composite by chemical methods. PEO-Au nanocomposites were prepared using the solution cast technique by dissolving PEO- Au nanocomposites in chloroform [113]. Subsequently, the electrospinning methodology was carried out to produce the nanofibers. They were able to achieve diameters in the range of $550 \mathrm{~nm}$. Although this process showed promise in forming fibers of two different materials, it did not form one single entity. Both materials, PEO and Au, continued to be separate elements in the fibers. Furthermore, the addition of caustic chemicals during the process might leave traces affecting cell growth. Moreover, this hybrid was not in nanoscale.

The fabrication of a scaffold should not only yield superior material characteristics but also structural properties that are favorable for the application. It has been shown that nanoscale 
biomaterials demonstrate greater competence compared to their micro and macro counterparts [72]. Nanoscale has been shown to have both cytotoxic as well as biocompatible reactions to cells. For instance, $\mathrm{Li}$ et al. presented the biocompatibility of electrospunnanofibrers[70]. On the other hand, Lee et al. showed that nanorods prevent cells from forming focal adhesions and subsequently kill them [114]. Consequently, it is seen that scaffold composition as well as nanostructure dictate the growth or death of cells.

In this chapter, a femtosecond laser synthesis method to fabricate a novel nanostructured hybrid material, made of nanofibers is introduced. These nanofibers are composed of pure gold, silicon oxide and an entirely new phase of gold-silicon $\mathrm{Au}_{3} \mathrm{Si}$ (212) that are generated directly from a gold coated silicon substrate. The process of fabrication of this nanofibrous matrix is explained by vapor-condensation method [59]. The effect of the new phase on mammalian cells was tested using mouse embryonic fibroblasts and mouse pre-osteoblasts. Fibroblasts are employed to study the cell-matrix interactions [115]. Osteoblasts were used to determine biocompatibility for orthopedic applications. This method of fabrication suggests significant promise in not only combining two elements but multiple elements as well. Furthermore, the use of immiscible elements is made possible using this method.

\subsection{Experimental Detail}

\subsubsection{MHz femtosecond laser synthesis}

Hybrid nanofibrous network of silicon and hybrid gold-silicon was directly patterned in a single step using a MHz femtosecond laser. Undoped silicon wafers $<100>$ with a thickness of $500 \mu \mathrm{m}$ (University Wafers, USA) was coated with gold (350nm in thickness) and were cut into $2 * 2 \mathrm{~cm}$ samples. These samples were washed with de-ionized (DI) water and ethanol and rinsed in DI water again. These samples were then irradiated using a diode pumped, Yb-doped femtosecond laser system (Clark-MXR Inc. IM-PULSE Series Ultrashort Pulse laser) at $26 \mathrm{MHz}$. The laser irradiates the samples in an array of points at a distance of $50 \mu \mathrm{m}$. The laser material interaction time was maintained at $0.1 \mathrm{~ms}$. The power of the laser determined the percentage of gold in the final hybrid nanofibrous structure. Three different powers were used $-16 \mathrm{~W}, 14 \mathrm{~W}$ and $12 \mathrm{~W}$. The laser pulse width was $214 \mathrm{fs}$ and wavelength was $1040 \mathrm{~nm}$. 


\subsubsection{Characterization of the hybrid structure}

Scanning electron microscopy (SEM) (Hitachi S 5200) was employed to study the morphology of the three dimensional nanofibrous structure. To study its nanoparticle composition and structure, transmission electron microscopy (TEM) (Hitachi H 700 CTEM) was used. X- Ray diffraction (XRD) (Siemens D5000 conventional theta/2theta diffractometer) was used to analyze the material composition/phase of the nanofibrous network. TEM was used in conjunction with Energy-dispersive X-ray spectroscopy (EDS) to assess the percentage of gold and silicon in the three dimensional nanofibrous structure. Atomic force microscopy (AFM) (NT-MDT) was performed to assess the adhesion forces on the nanofibrous structure. A single crystal silicon tip (antimony doped) with a spring constant of $0.11 \mathrm{~N} / \mathrm{m}$ was employed in contact mode. NT-MDT software was used to calculate the force of adhesion from the obtained values.

Zeta potentials were measured using zetasizer (Nano-ZS90, Malvern Instruments). The nanofibrous structures were scratched off the surface uniformly and were mixed in $1 \mathrm{ml}$ of deionized water and transferred to a cuvette, after which measurements were taken.

\subsubsection{Cell-biomaterial interaction}

Methods mentioned in chapter 3 were employed to study cell biomaterial interaction.

\subsubsection{Statistics}

All experiments were performed in triplicates. Averages and standard deviations were calculated from combined results.

\subsection{Results and Discussion}

\subsubsection{Synthesis of hybrid gold-silicon nanostructures}

The formation of the alloy like hybrid gold-silicon structure can be explained by the processes of nucleation and condensation. When the femtosecond laser interacts with the surface of gold coated silicon, the energy is instantaneously absorbed by the conduction band electrons. This results in an increase in temperature near the surface. The ultrashort laser pulse increases the temperature of the electrons at the surface and leaves the lattice comparatively cooler as the electrons at the top have lesser heat capacity. There is a gradual energy transfer from the electrons to the lattice due to electron-phonon coupling. At a repetition rate of $26 \mathrm{MHz}$, the 
pulses alter the gold film and consequently increase absorption of the gold surface. The subsequent formation of nanostructures is attributed to nucleation and condensation. Figure 4-1 illustrates the complete process of synthesis and application.
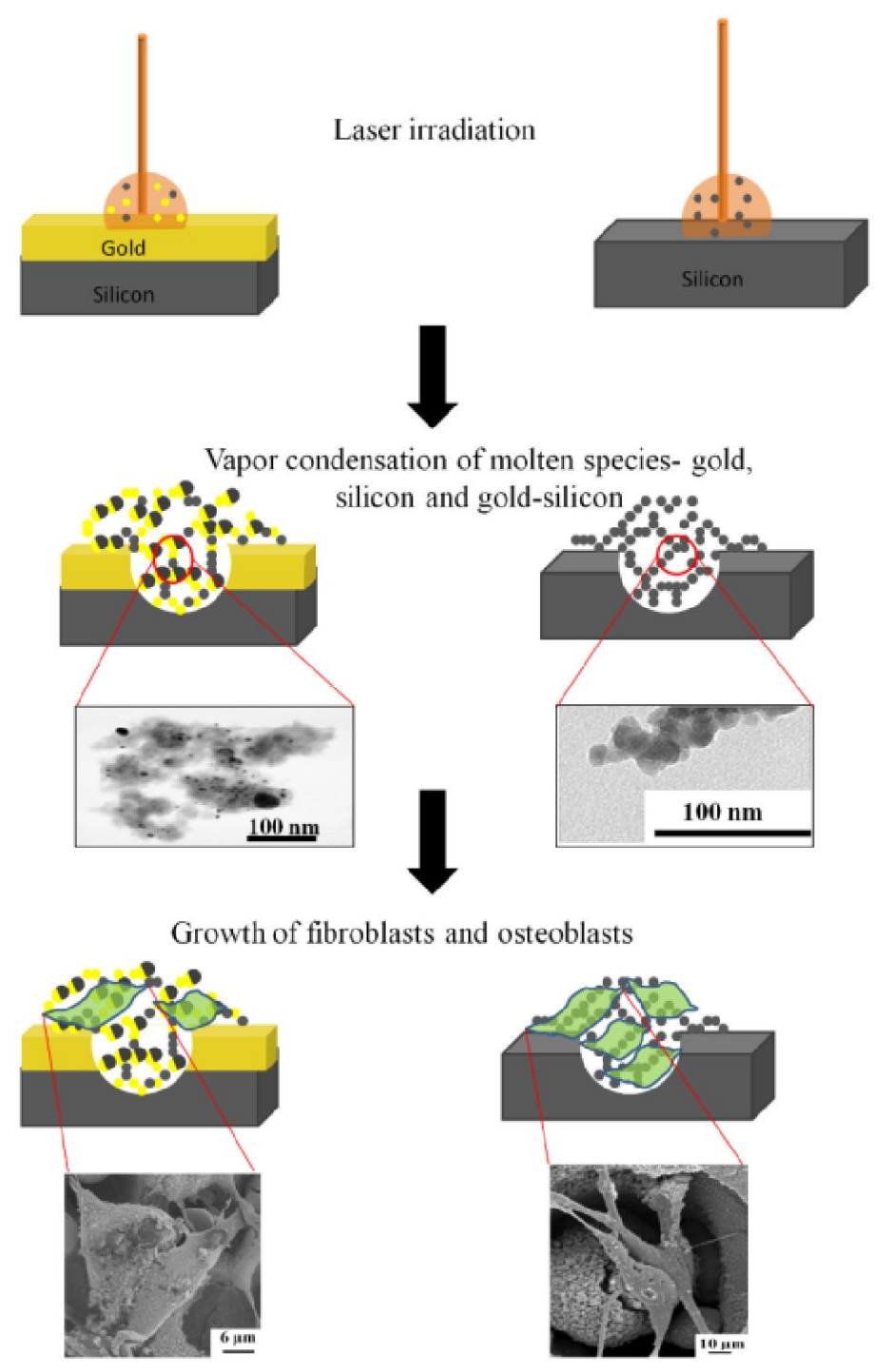

Figure 4-1: Illustration of the gold-silicon Vs Silicon nanofibers synthesis and application.

\subsubsection{Characterization of the fabricated nanostructures}

Figure $4-2(a \& c)$ shows a three dimensional gold-silicon nanostructure. This is a hybrid of both silicon and gold nanoparticles whereas the plain silicon nanostructure consists of only silicon and silicon oxide nanoparticles (Figure 4-2(b\&d)). The nanostructure is highly porous with varying pore sizes. The diameters of the fibers possess substantial uniformity. The presence of both gold 
and silicon nanoparticles is confirmed from TEM and EDX images (Figure 4-3). The image also demonstrates the increase in gold with lower powers. The dark spheres in the image correspond to gold.
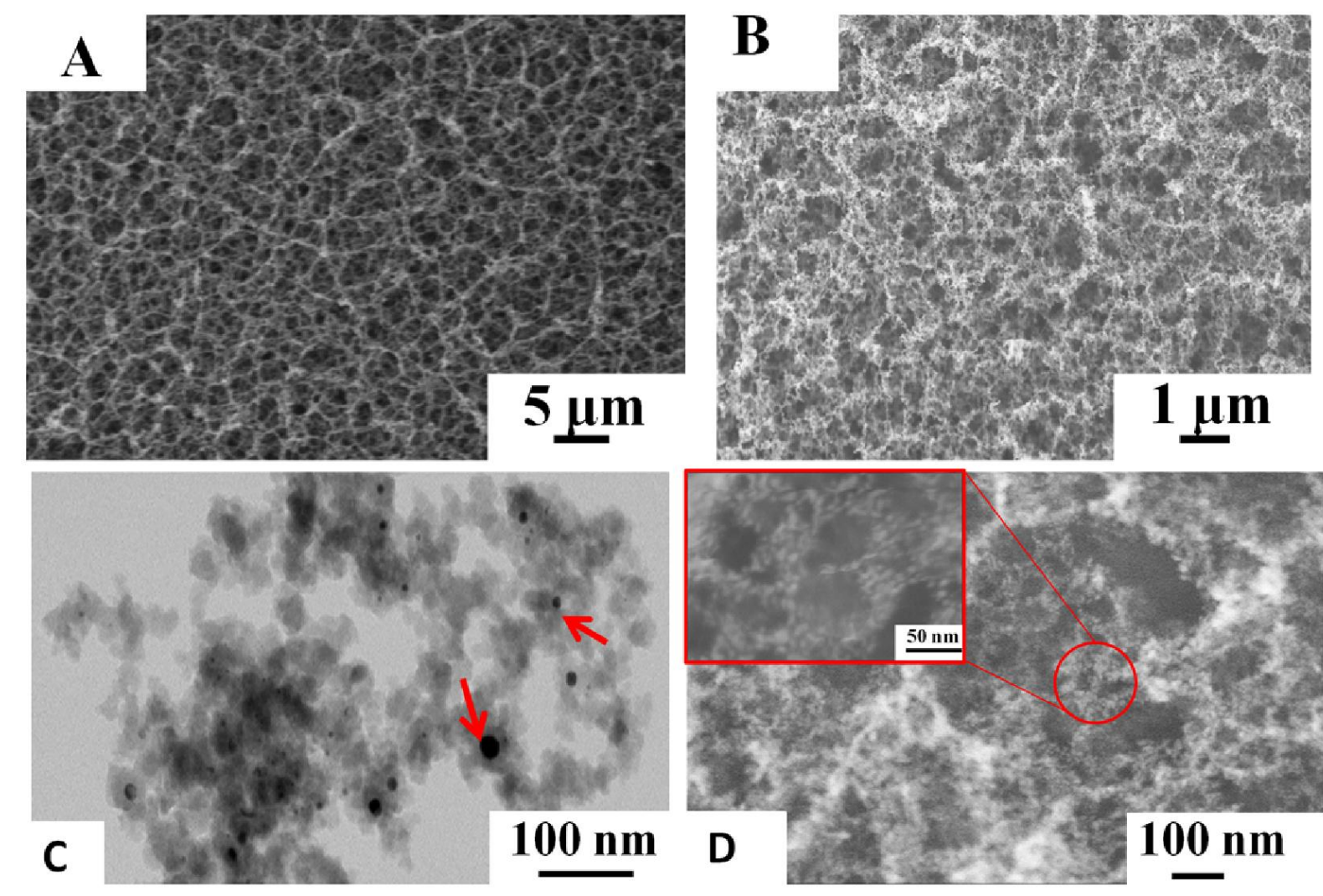

Figure 4-2: SEM image of a three dimensional porous alloy like hybrid gold-silicon nanostructure and plain silicon nanostructure. (c) TEM of gold-silicon nanostructure. The red arrows indicate gold nanoparticles or gold-silicon nanoparticles. (d) SEM micrograph of plain silicon nanostructure.

EDX analysis shows the gold-containing spheres also contain silicon oxide. The nanoparticles on the whole are attached together by strong bonds giving the appearance of nanofibers. There is also the presence of non-fused gold nanoparticles in this nanostructure. The average size of the gold, silicon and gold-silicon nanoparticle was controllable using parameters like power, dwell time and interaction time. From the TEM images, it was seen that there was a bimodal distribution of nanoparticles. The interaction time of the laser irradiating the surface played a vital role. At an interaction time of 1.30 minutes, the majority of nanoparticles had a size of 2$10 \mathrm{~nm}$. The bigger nanoparticles varied between $37-49 \mathrm{~nm}$. It was noticed that as the interaction 
time increased, the number of nanoparticles in the 2-10nm range reduced and nanoparticles in the 37-49nm range increased. Additionally, the larger particles also increased in size with increase in interaction time. The pores of the hybrid were found to be $20-35 \mu \mathrm{m}$ on the top layer. The percentage of gold in the entity was controlled by varying the power of the laser. A percentage in the range of 35-78 was achieved with different powers. Figure 4-3 clearly illustrates a constant ratio between silicon and oxygen content in the nanostructured hybrid. This proves that vapor condensation mechanism plays a dominant role during formation [116]. The melting point of gold is $1063^{\circ} \mathrm{C}$ and is $1414^{\circ} \mathrm{C}$ for silicon. This variation in melting point results in different particle sizes. Since silicon has a higher melting point, its nucleation stops before that of gold, resulting in smaller silicon nanoparticles. The other variation was in the concentration of gold in the nanostructure. This was due to a change in average pulse energy. $E_{a}=P / f$, where $P$ is power and $\mathrm{f}$ is frequency of pulses. A decrease in power therefore decreases average pulse energy. This causes a change in the volume of silicon and gold species being ablated into the plume. When laser power increases, there is an increase in ablated solid volume. However, when ablated volume increases, the gold content remains constant since the thickness of the gold layer in the order of $100 \mathrm{~nm}$, is much smaller compared to the thickness of the silicon substrate. Therefore a decrease in gold concentration is observed with an increase in laser power.

Phase analysis of this hybrid nanostructure showed interesting results. Varying the laser power resulted in different phases of the gold-silicon. Figure 4-4 shows an XRD micrograph depicting the $\mathrm{Au}-\mathrm{Si}, \mathrm{SiO}_{2}$ and pure gold in the fabricated nanostructures (Au81Si19 (JCPD 39-0735)).

The material composition has an effect on its surface interaction. To measure surface characteristics, force of adhesion and zeta potentials was measured using an atomic force microscope (AFM) and a zetasizer, respectively. The AFM cantilever was brought close to the substrate and then retracted. Forces of adhesion of the gold-silicon hybrid can be calculated from the force curve using, $\mathrm{F}=\mathrm{k}^{*} \Delta$ lwhere, $\mathrm{F}$ is the adhesion force $(\mathrm{nN}), \mathrm{k}$ is the spring constant $(0.11$ $\mathrm{N} / \mathrm{m}$ ) of the cantilever and $\Delta \mathrm{l}$ is the deflection distance (nm) [67]. Figure 4-5 (a) shows the effect of gold concentration on the adhesion force. 

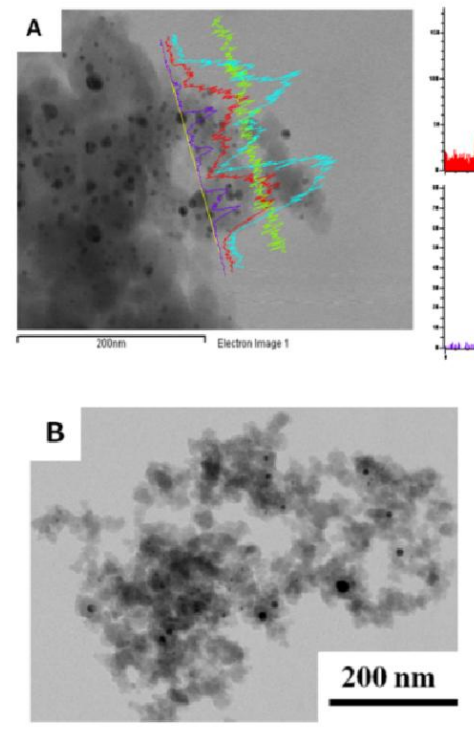

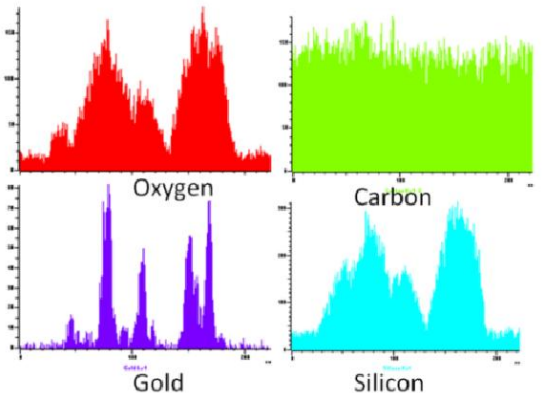

$c$

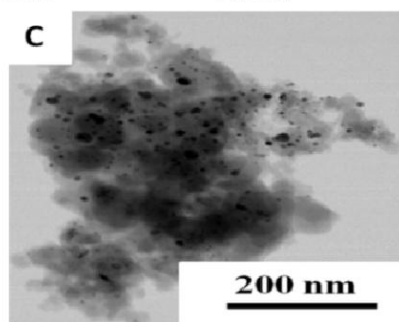

Figure 4-3: (a) TEM image and the corresponding EDX graphs of hybrid gold-silicon (b) TEM of hybrid gold-silicon fabricated at higher power demonstrating lower percentage of gold (c) TEM of hybrid gold-silicon fabricated at lower power demonstrating higher percent percentage of gold to silicon. Darker shades show gold nanoparticles.
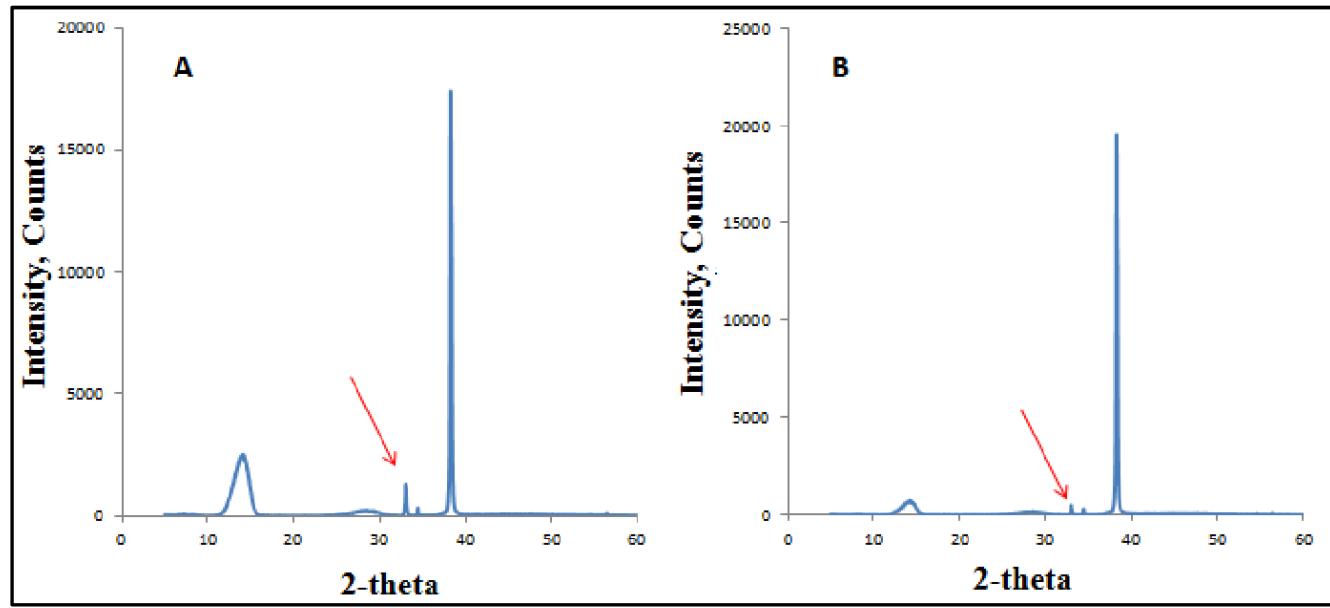

Figure 4-4: The XRD micrographs depict the material composition of the gold-silicon hybrid nanostructures fabricated at (a) $78 \%$ gold (b) $35 \%$ gold

It is seen that there is a significant decrease in force of adhesion between $61 \%$ and $78 \%$ of gold concentration. This implies that higher concentration of silicon plays a role in increasing 
adhesion forces. Our previous research shows that plain silicon nanostructures have significantly high adhesion forces [94]. Zeta potentials of the gold-silicon hybrid nanostructures showed similar results (Figure 4-5 (b)). A concentration of $78 \%$ gold showed only $-1.8 \mathrm{mV}$ zeta potential whereas $61 \%$ gold displayed $-4.5 \mathrm{mV}$ zeta potential. These studies clearly demonstrate the effect of gold concentration on surface interaction properties of the gold-silicon hybrid nanostructure.

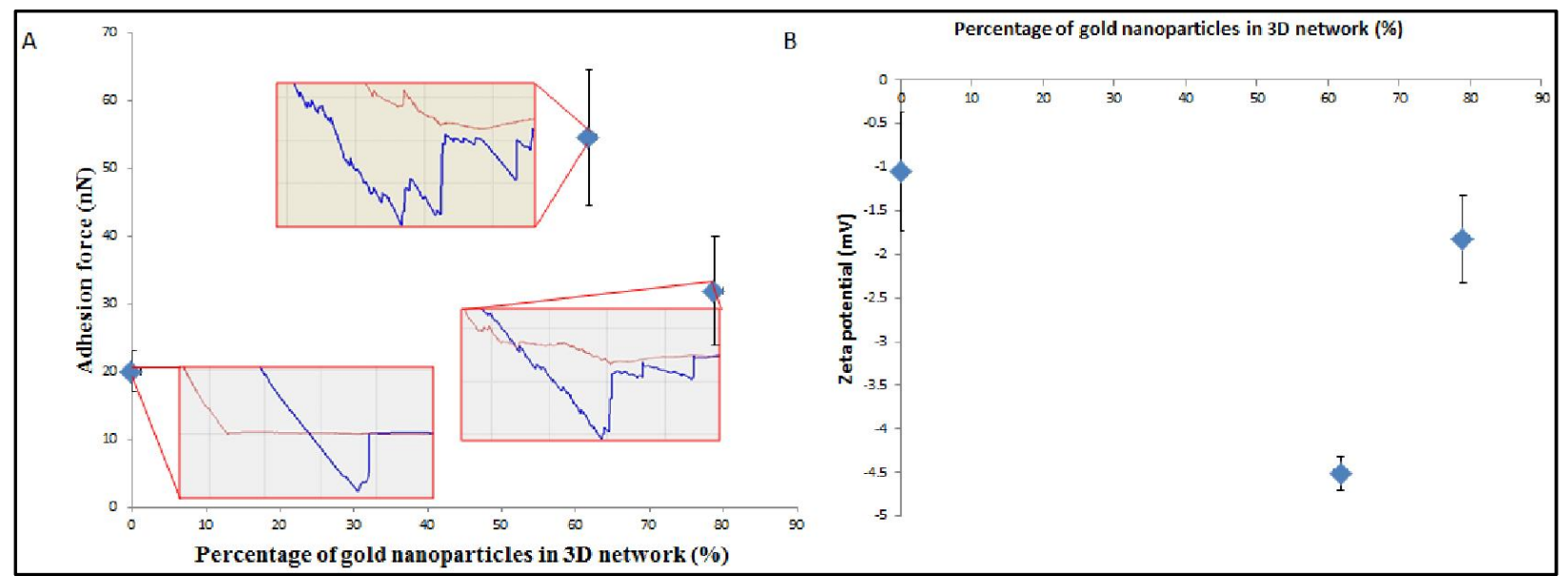

Figure 4-5: (a) The adhesion forces of fabricated gold-silicon alloy like hybrid nanostructures shows higher percentage of gold has lower adhesion force. The inset images show representative adhesion curves at each data point (b) The zeta potentials show a similar trend to the adhesion forces where higher percentage of gold has lower zeta potentials.

The concentration of gold and the size of the nanoparticles lead to varying zeta potentials and adhesion forces. As seen from Figure 2 and 3, gold nanoparticles have a bigger size compared to silicon nanoparticles. This increase in size of gold nanoparticles maybe a possible reason for decrease in adhesion forces and zeta potentials compared to plain silicon nanofibrous network.

\subsubsection{Controlling the growth of fibroblasts and osteoblasts}

Our previous research with silicon nanostructures showed a marked increase in fibroblastic growth [94]. In an attempt to effectively control cell growth, the concept of varying percentages of gold within the nanostructure is introduced. To study this effect of different cell lines, both mouse embryonic fibroblasts and mouse calvaria-derived pre-osteoblastic cells were employed. Proliferation of these cells was studied by conducting an MTT assay. Cell morphology 
characteristics were assessed using scanning electron microscopy (Figure 4-6(a-b)). Figure 4-6(c) details the effects of gold concentration on cell proliferation. There was an increase in cell proliferation with decrease in gold concentration. The increase was more pronounced for fibroblasts in comparison with osteoblasts. $100 \%$ gold refers to gold control samples. At $65 \%$ and $35 \%$, the cell proliferation seems to form a plateau. To further understand the growth of both osteoblasts and fibroblasts, SEM images were used to study the morphological characteristics (Figure 4-6(d)). Since both cells seemed to prefer higher concentrations of silicon, the morphology of cells on gold-silicon hybrid nanostructures was compared to plain silicon nanostructures. Although fibroblasts proliferated faster than osteoblasts, the osteoblasts tended to spread out on the $\mathrm{X}$ axis more. It is seen that osteoblasts spread significantly more on silicon surfaces compared to gold-silicon nanostructure on the $\mathrm{x}$ axis. The spreading of fibroblasts on the $\mathrm{x}$ axis was not significantly different on gold-silicon and plain silicon surfaces. Furthermore, spreading on the y axis was not significantly different on all surfaces either.

The growth of cells on these surfaces showed interesting results. The reduction in proliferation of both osteoblasts and fibroblasts is attributed to the increase in gold concentration. Pan et al. suggested that gold nanoparticles are cytotoxic to cells [110]. This may be a possible reason for reduction in cell growth. In the nanostructure, in addition to gold silicides, there is also a presence of non-fused gold. During cell growth, it is possible that the gold and silicon separate and the gold nanoparticles enter the cell via pinocytosis [111]. Additionally, the phase of the nanostructure also plays a role in cell growth. The gold-silicon phase formed is $\mathrm{Au}_{3} \mathrm{Si}$ (212) [117]. This is seen in the phase micrograph from Figure 4-4. This XRD was also compared to plain silicon nanofibrous network to confirm the presence of gold silicide. The intensity of this phase was significantly lesser when more energy was used. The sizes of these nanoparticles also play a role. Protein adsorption is higher on smaller nanoparticles. The formation of gold-silicon gave rise to larger nanoparticles and this may in turn lead to lesser protein adsorption, consequently affecting cell growth [118]. Due to the same reasons, the osteoblasts spread out more on the plain silicon nanostructures in comparison to the gold-silicon alloy like hybrid. 


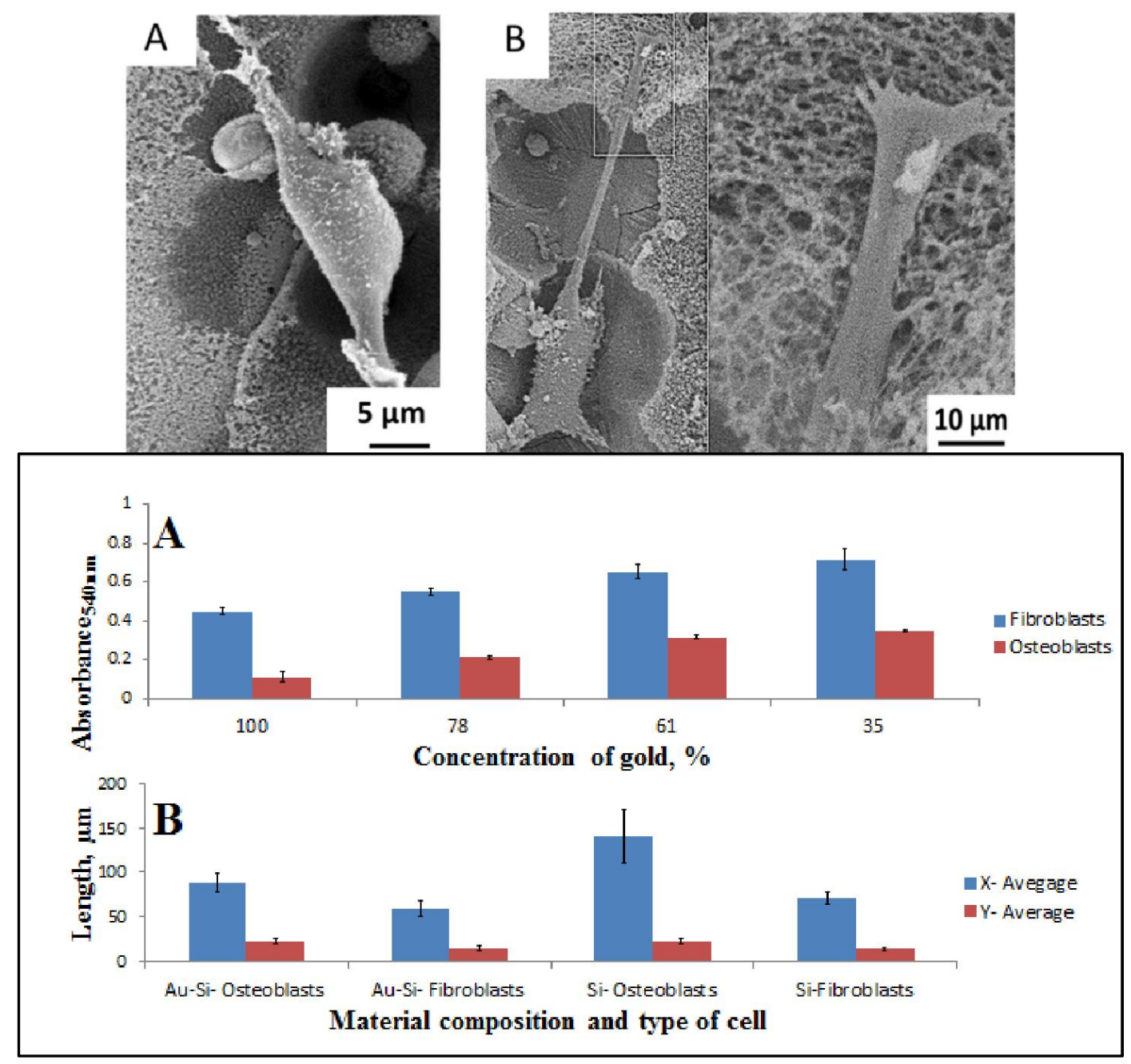

Figure 4-6: (a) SEM image of a fibroblast growth on gold-silicon alloy like hybrid nanostructure.

(b) Growth of fibroblast on plain silicon nanostructure. The interaction of the cell with the nanofibers is seen on the magnified image on the right. (c) effect of concentration of gold in gold-silicon alloy like hybrid on fibroblast and osteoblast proliferation (d) cell adhesion morphology on gold-silicon and plain silicon nanostructures.

\subsection{Summary}

For the first time, a novel femtosecond synthesis method to fabricate a nanostructured hybrid of two immiscible elements- gold and silicon for tissue engineering applications is introduced. A new phase, $\mathrm{Au}_{3} \mathrm{Si}$ is presented here. Previous studies showed only bulk or macro/micro structured hybrids. Materials like gold which are highly inert and stable were difficult to 
nanostructure. This method allowed not only a new alloy phase but also allowed for the change in concentration of gold. The concentration of gold and the particle sizes are entirely controlled by the laser parameters. The particle sizes in this nanostructure range between $2-10 \mathrm{~nm}$ to 37 $49 \mathrm{~nm}$. Pore sizes of $20-35 \mu \mathrm{m}$ are formed on the top layer. The effect of this new phase was tested on mammalian cells. Growth and adhesion characteristics of fibroblasts and osteoblasts demonstrate high level of controllability. Immense potential for this method of fabrication and the novel hybrid nanostructures is perceived. The possibility of alloying two or more immiscible elements in a new phase and in nanoscale presents opportunities in fields like biosensing and cancer research. 


\section{Chapter 5}

\section{Programming cancer through phase transformed functionalized silicon based biomaterials}

The most vital aspect of tissue engineering is to enhance biocompatibility while preventing undesirable growth of cells. The need to direct and manipulate the growth of cells is paramount in tissue engineering as well as in point of care diagnostics and lab-on-a-chip devices. However, controlling the growth of cells on the surface of silicon without any additive layer or topographical modification is still unexplored. This chapter delineates the discovery of unique properties of a bio-functionalized silicon substrate, programmed to completely repel or control cells, generated by ultrafast pulse interaction with silicon in the femtosecond regime. Remarkably, bio-functionalization without changes in topology or morphology is observed indicating only sub-surface phase transformations. Further, this versatile technique permits programming the substrate in any shape or size, according to its application. Material characterization reveals the presence of a unique mixture of various phases of $\mathrm{SiO}_{2}$ as well as $\mathrm{Si}$. Phases such as stishovite that are conventionally unstable at ambient temperatures are detected on these surfaces. The culture of mouse embryonic fibroblasts show unique adhesion characteristics on these phase transformed silicon surfaces. Cell controlling, trapping, shaping is precisely achieved. Furthermore, the directionality of fibroblasts is restrained and can be consequently changed. The precise controlling of growth, proliferation, migration and adhesion of cells is attributed to the unique phase transformation of silicon, accomplished by reprogramming inherently biocompatible silicon into a cell repelling surface. Cell repelling on silicon has not been reported previously and presents considerable promise in a myriad of applications. Key areas such as tissue engineering, MEMS, lab on a chip devices as well as antifouling applications necessitate further research on such surfaces. 


\subsection{Introduction}

Cell-biomaterial interaction is an intricate phenomenon. Advancements in this field facilitate the fabrication of highly functional biomaterials. Silicon is a highly sought after biomaterial due to its versatility and ease of manipulation owing to its unprecedented use in the micro-fabrication industry[94], [119]-[122]. However, a significant portion of research is tailored towards cell proliferation as studies have proven silicon's innate biocompatible nature. While this conservative outlook has witnessed several vital innovations, it has been lacking research in the field of directing and controlling the growth of cells. Controlling the shape, direction and adhesion of cells is a vital area of research. Our discovery of sub-surface modified silicon generating cell repelling properties has created a paradigm shift in the way tissue engineering, lab on a chip, diagnostic devices are perceived.

Researchers in the past have attempted to change the characteristics of silicon to control the growth of cells. These are limited to either topographical or chemical modifications [123]. Lithography and photolithography are primary techniques that generate topographical changes[124]. For instance, Curtis et al. reviewed the effects of silicon groove-ridge topographies on cell growth [125]. Parameters such as height, depth, range and roughness effected cell growth.Topographical modifications demand elaborate, expensive setups and are highly dependent on the wavelength of the light used. As a result, feature size is a concern. Inexact feature sizes will negate precisely controlled cell growth.

Chemical modificationentails changes in hydrophobicity/hydrophilicity, oxide layers, functionalization of surfaces and surface energy. Ranella et al. studied the effects of wettability, oxidation on silicon [31]. In their study, silicon surfaces were irradiated with a laser at a pressure of 10-2 mbar. The resultant spikes were coated with a hydrophilic oxide layer or a hydrophobic silane layer. The hydrophilic oxide surfaces showed more cell attachment. On the contrary, hydrophobic silane surfaces changed the morphology of cells from flat to rounded indicating less adhesion.In a study pertaining to surface functionalization,Sharma et al. coupled silicon with Poly (ethylene glycol) in order to convert the biocompatibility of silicon into functionalized surfaces that prevent protein adsorption and cell growth[126]. Thusly, it is observed that both chemical and topographical modifications completely alter the surface of silicon. Moreover,chemical modifications may introduce unknown toxins to a biological environment. 
The need to control the growth, migration and adhesion of the cells is necessitated by specialized applications such as implants and micro devices. For instance, cardiovascular stents require enhanced cell controllability [127]. Long term stability of these implants in the body requires specific augmentation or prevention of smooth and epithelial cell growth. Furthermore, with respect to neural cells, directed growth is a priority in order to mimic its growth in an in-vivo environment[128]. Additionally, devices such as biosensors and cell based sensors require an elevated level of precision with respect to cell growth. Micro/nano diagnostic devices routinely require that cells flow in a specified direction, within narrow channels [129]. Likewise, certain applications require that singular cells be trapped for investigation [130,131].

A unique method to program silicon, converting it from an innately proliferative surface to a repelling surface is introduced (Figure 5-1). Ultrafast pulses coupled with femtosecond pulse duration initiate sub-surface modifications along with the formation of novel phases of silicon. The versatility of the laser permits the synthesis of cell repelling, cell isolating, cell channeling and direction controlled cell migrating surfaces. The peak power of the laser is varied while the frequency of pulses is maintained. Results indicate that the highest peak power displayed superior cell repelling properties. Furthermore, highest peak power also showed most precise controlling of shape, proliferation, migration and directionality.

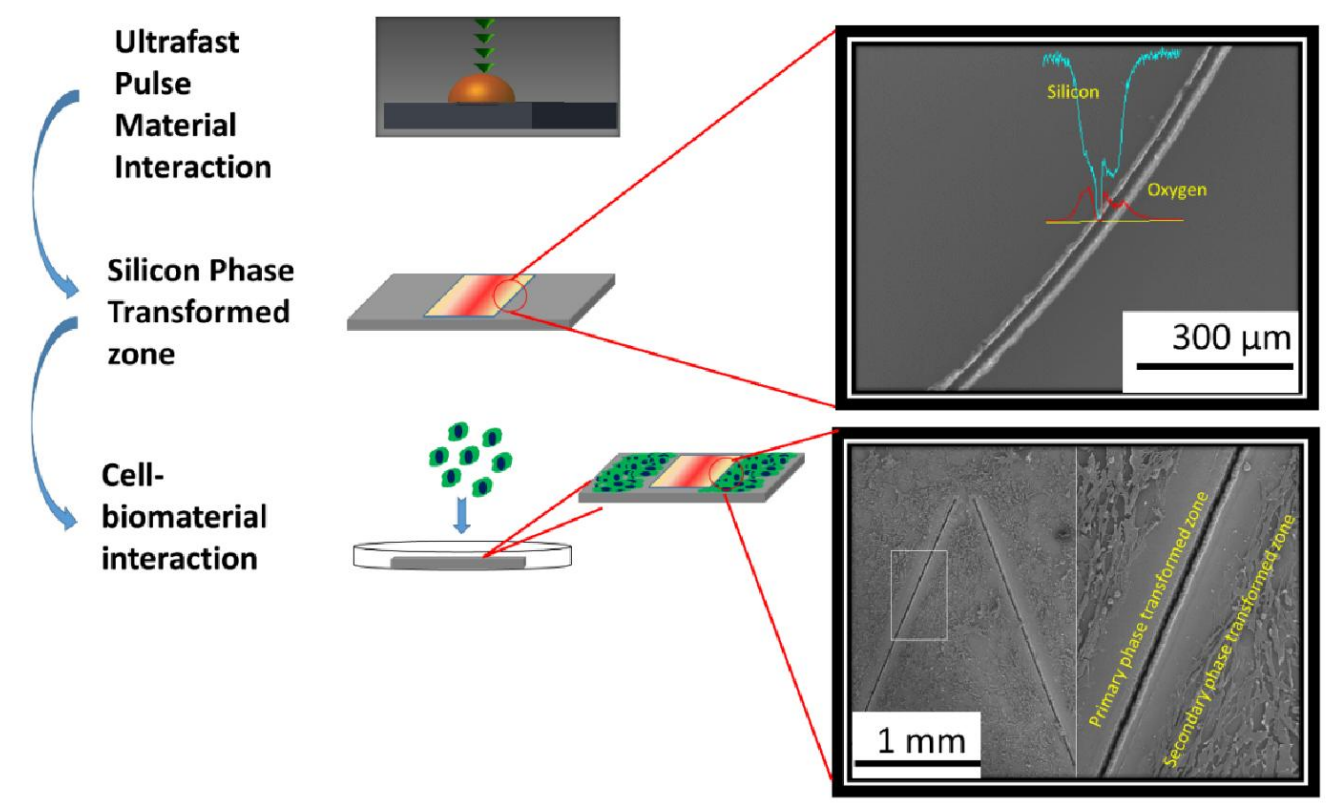

Figure 5-1: Illustration fabrication and application of phase transformed cell repellant zones. 


\subsection{Experimental Detail}

\subsubsection{Ultrafast laser synthesis}

Sub-surface modification of silicon is synthesized in a single step using a $\mathrm{MHz}$ femtosecond laser. Undoped silicon wafers $<100>$ with a thickness of $500 \mu \mathrm{m}$ (University Wafers, USA) and are cut into $2 * 2 \mathrm{~cm}$ samples. These samples are washed with de-ionized (DI) water and ethanol and rinsed in DI water again. These samples are then irradiated using a diode pumped, Yb-doped femtosecond laser system (Clark-MXR Inc. IM-PULSE Series Ultrashort Pulse laser) at $8 \mathrm{MHz}$. The laser irradiates the samples in an array of lines at distances between $100 \mu \mathrm{m}$ to $2 \mathrm{~mm}$. Further, different shapes to simulate tissue engineering and diagnostic device environments are synthesized. The speed at which the laser irradiates and the power of the laser is maintained at $10 \mathrm{~mm} / \mathrm{s}$ and $15 \mathrm{~W}$ respectively. The laser pulse width or peak power plays an important role and is varied between $214 \mathrm{fs}, 714 \mathrm{fs}$ and $1428 \mathrm{fs}$ corresponding to $8.76 \mathrm{MW}, 2 \mathrm{MW}$ and $1.31 \mathrm{MW}$ respectively. All the parameters are controlled by a computer to facilitate precision and accuracy.

\subsubsection{Characterization of phase transformed surfaces}

Scanning electron microscopy (SEM) (Hitachi S 5200) is used to characterize the morphology of the phase transformed silicon surfaces. The samples are loaded onto aluminum stands and gold coated for SEM. Following SEM, Energy-dispersive X-ray spectroscopy (EDX) is carried out to determine the elemental composition of irradiated silicon. Micro-raman spectroscopy as well as X-Ray photon spectroscopy (Thermo scientific K-alpha) is further used to study surface composition.

Change in the phase of silicon is observed using an atomic force microscope (AFM)(NT-MDT, Russia). A single crystal silicon tip (antimony doped) with a spring constant of $0.11 \mathrm{~N} / \mathrm{m}$ is employed in semi-contact mode to measure phase contrast on silicon surfaces.

\subsubsection{Cell growth and culture}

Mouse embryonic fibroblasts (NIH 3T3, ATCC, USA) are employed to qualitatively and quantitatively study cell controllability. Passages between 3 and 6 were used for cell studies. The irradiated substrates are washed with alcohol and D.I. water and kept under UV light for 20 
minutes. Subsequently, the substrates are placed in petri dishes with fibroblasts at a density of $10^{5}$ cells $/ \mathrm{ml}$. The petri dishes are placed in an incubator for 24 hours. After the incubation period the samples are fixed in $2 \%$ glutaraldehyde in $0.1 \mathrm{M}$ sodium cacodylate buffer $\mathrm{pH} 7.3$ for an hour. Next, the samples are immersed in $0.1 \mathrm{M}$ sodium cacodylate buffer with $0.2 \mathrm{M}$ sucrose $\mathrm{pH}$ 7.3 for 20 minutes. Dehydration in increasing concentrations of alcohol for 20 minutes each is followed. The samples are then critical point dried and imaged. Fluorescence microscopy is conducted to study adhesion characteristics of fibroblasts. The samples are first fixed in methanol free paraformaldehyde followed by incubation with milk to prevent non-specific binding. To stain the actin and cytoskeleton, the samples are incubated with Alexa fluor 488(Life Technologies) followed by DAPI (Life Technologies) to stain the nucleus. The samples are studied using a fluorescence microscope (Nikon).

\subsubsection{Statistics}

All experimentsare carried out in triplicates and the data points are averages unless otherwise mentioned. The error bars indicate standard deviations.

\subsection{Results and Discussion}

\subsubsection{Synthesis of Cell controlling regions}

Silicon samples are irradiated with ultrafast pulses in the femtosecond regime. The peak power determines the interaction between pulses and the silicon surface.An increase in peak power results in the formation of significantly larger areas of phase transformed silicon. This change in synthesis of phase transformation is attributed to difference in temperature at different peak powers, after each pulse [132]. The temperature is calculated as

$$
\mathrm{T}=\sqrt{\frac{2 \mathrm{a}}{\pi^{3} t_{p}}} \frac{4 K(1-R) P}{k^{2} d^{2}}
$$

wheret $_{\mathrm{p}}$ is the pulse duration, $\mathrm{a}$ is the thermal diffusion coefficient, $\mathrm{k}$ is the heat conduction coefficient, $\mathrm{R}$ is the reflection coefficient, $\mathrm{K}$ is the residual energy coefficient, $\mathrm{P}$ is laser power, $\mathrm{f}$ is frequency of pulses and $\mathrm{d}$ is the laser spot diameter. A temperature of $7935 \mathrm{~K}, 4181 \mathrm{~K}$ and $3072 \mathrm{~K}$ is calculated for peak powers of $8.76 \mathrm{MW}, 2 \mathrm{MW}$ and $1.31 \mathrm{MW}$. These high temperatures result in the formation of new oxides, change in physicochemical characteristics and changes in the crystal lattice of silicon. This research has focussed on the effect of peak power based on 
change in pulse width in the femtosecond regime. At lower pulse widths local energy absorption occurs whereas at higher pulse widths, the laser-excited electrons transfer their energy to the lattice. At constant power, the surface temperature is proportional to pulse duration, thusly an increase in pulse duration results in increased surface temperature [133]. It is expected that at higher pulse durations, the heat travels in the z-axis (depth) and the length of the pulse contributes to a steady state thermal propagation.

In this study, the surface temperature as well as the temperature gradient is believed to play a role in phase transformation, including the formation of various silicon polymorphs such as tridymite. The formation of silicon polymorphs could be attributed to pressure induced changes or altered laser-material interaction owing to raster scanning.Furthermore, heat affected zones of femtosecond duration pulses initiate temperature increases several micrometers deep. These temperature increases initiate plastic deformation mechanisms in silicon [134]. All of these phase transformations are precisely controlled owing to high peak power and multiple short interval pulses.

\subsubsection{Characterization and analysis of cell controlling regions}

Surface phase transformation is observed using scanning electron microscopy (SEM) and Energy-dispersive X-ray spectroscopy (EDX) (Figure 5-2 (A-B)). Various shapes were fabricated to study characteristics of cell trapping, isolation and channeling, mirroring research needs in areas of tissue engineering and diagnostic devices. Results indicate the presence of a distinct phase transformed zone. The width of this phase transformed zone decreases with decrease in peak power from $8.76 \mathrm{MW}$ to $1.31 \mathrm{MW}$. It is apparent that heat generated at higher peak powers travelled laterally as opposed to travelling to the lattice. Furthermore, the phase transformed zone is divided into two zones, the primary and secondary cell controlling regions. EDX analysis on the primary and secondary phase transformed zones demonstrates the presence of silicon and oxygen (Figure 5-2(A-B)). 

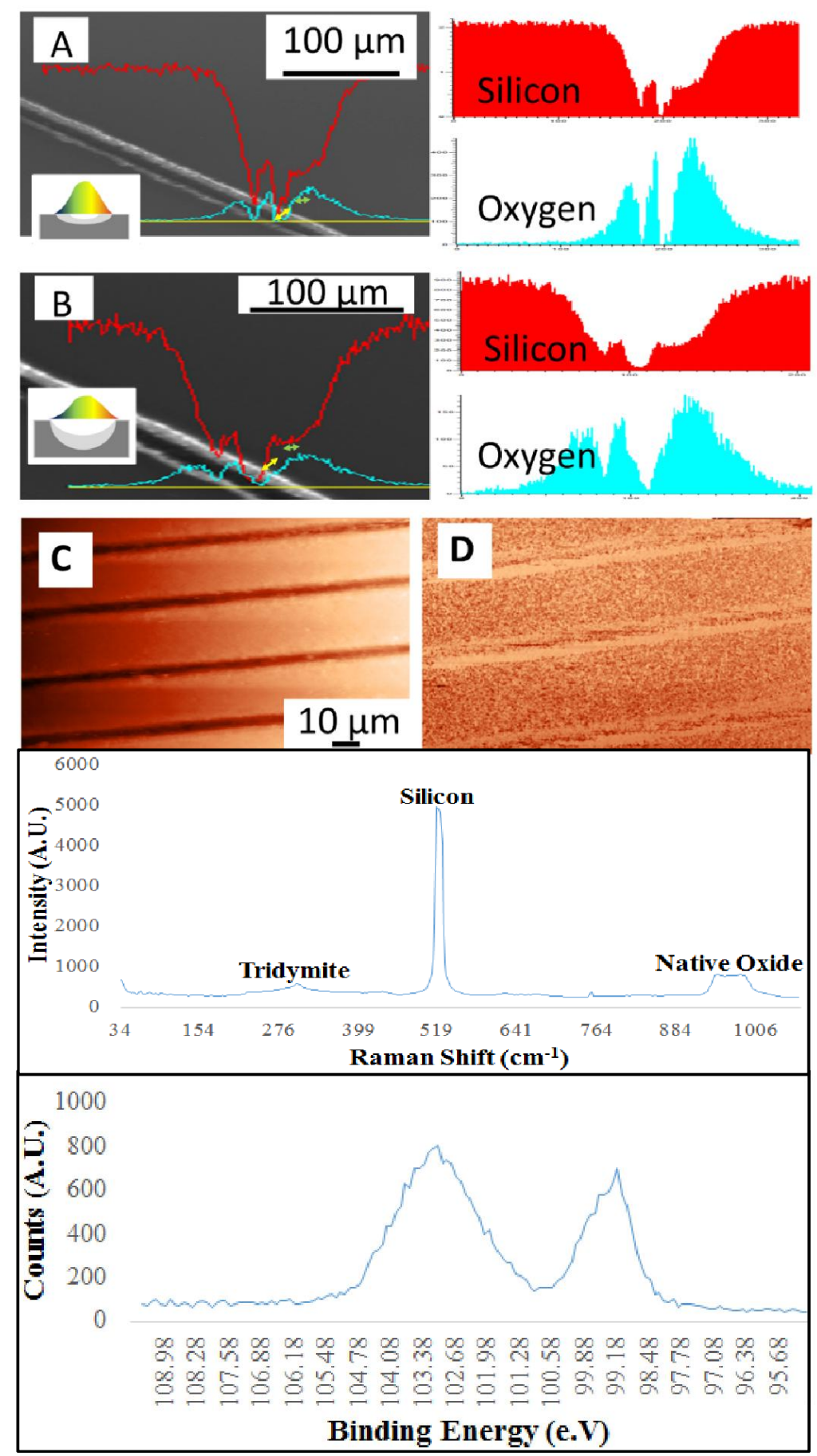

Figure 5-2: EDX images of phase transformed zones (A) At 8.76MW (B) 1.31 MW. The yellow arrow shows primary transformed zone and green star denotes secondary transformed zone. (C) AFM micrograph in height contrast (D) AFM micrograph in phase contrast mode (E) micro Raman analysis (F) XPS measurements 
It is observed that the ratio of oxygen to silicon varies with increase in distance from lasermaterial interaction point. Oxygen content is highest in the primary phase transformed zone and then decreases as it approaches the secondary phase transformed zone. This change in oxygen content is ascribed to the temperature gradient generated at each peak power. The primary phase transformed zone consists of high temperature phases comprising higher oxygen levels whereas the secondary phase transformed zone, with lower oxygen content is made of low temperature phases.

To further analyze the primary and secondary phase transformed zones atomic force microscopy was carried out in contact mode. This method is able to accurately detect any difference in phase including physico-chemical characteristics. Figure 5-2 (C-D) shows the topography as well as phases of the ultrafast pulse irradiated surface. It is clearly illustrated that the areas surrounding the ultrafast pulse irradiated point consists of a different phase when compared to bulk silicon. Phase contrast microscopy relies on the phase lag between the cantilever oscillation and cantilever's piezo driver. This phase shift on an AFM detects variations in materials. Boussu et al. demonstrated the relationship between phase shift and the hydrophobic/hydrophilic nature of the material [135]. On the phase transformed zones there isa $18^{\circ}$ phase shift on average. Since the tip used in the experiment is hydrophilic, it can be concluded that the new phases are hydrophobic in nature. Further, it has been stated that heating changes a hydrophilic surface to a hydrophobic surface, thus affirming aforementioned results [136].

While AFM confirmed the presence of a different phase, micro-Raman and XPS were used to qualitatively analyze these phases (Figure 5-2(E-F)). Micro-Raman analysis revealed three consistent peaks at all peak powers. The major sharp peak seen at $520 \mathrm{~cm}^{-1}$ corresponds to silicon. A very broad peak at $945 \mathrm{~cm}^{-1}$ corresponds to the native oxide layer on silicon. A unique, sharp peak is observed at $304 \mathrm{~cm}^{-1}$ corresponding to a crystalline polymorph of silicon oxide namely, monoclinic tridymite[137]. Similar results were obtained with XPS analysis. There is a presence of two significant peaks around $99 \mathrm{eV}$ and $103 \mathrm{eV}$, representing substrate silicon and silicon oxides respectively $[138,139]$. It is observed that at lower peak powers, the oxide peak is significantly higher than the substrate silicon peak. Furthermore, a decrease in peak power resulted in the oxide peak shifting towards lower binding energies.This decrease can be 
attributed to reduction of $\mathrm{SiO}_{2}$ to immediate oxidation states of silicon. Atoms of lower positive oxidation will exhibit lower binding energy.

\subsubsection{Cell culture studies}

Cell trapping, directional control and cell repelling is observed in this research study. Each of these phenomenonoccur corresponding to different interaction mechanisms of the ultrafast femtosecond pulses and silicon. The precise generation of heat on the surface of silicon permits controllability at a single and multiple cell level. The promotion of high and low temperature phases are contributing factors. Figure 5-3 illustrates various key research areas probed in this study.

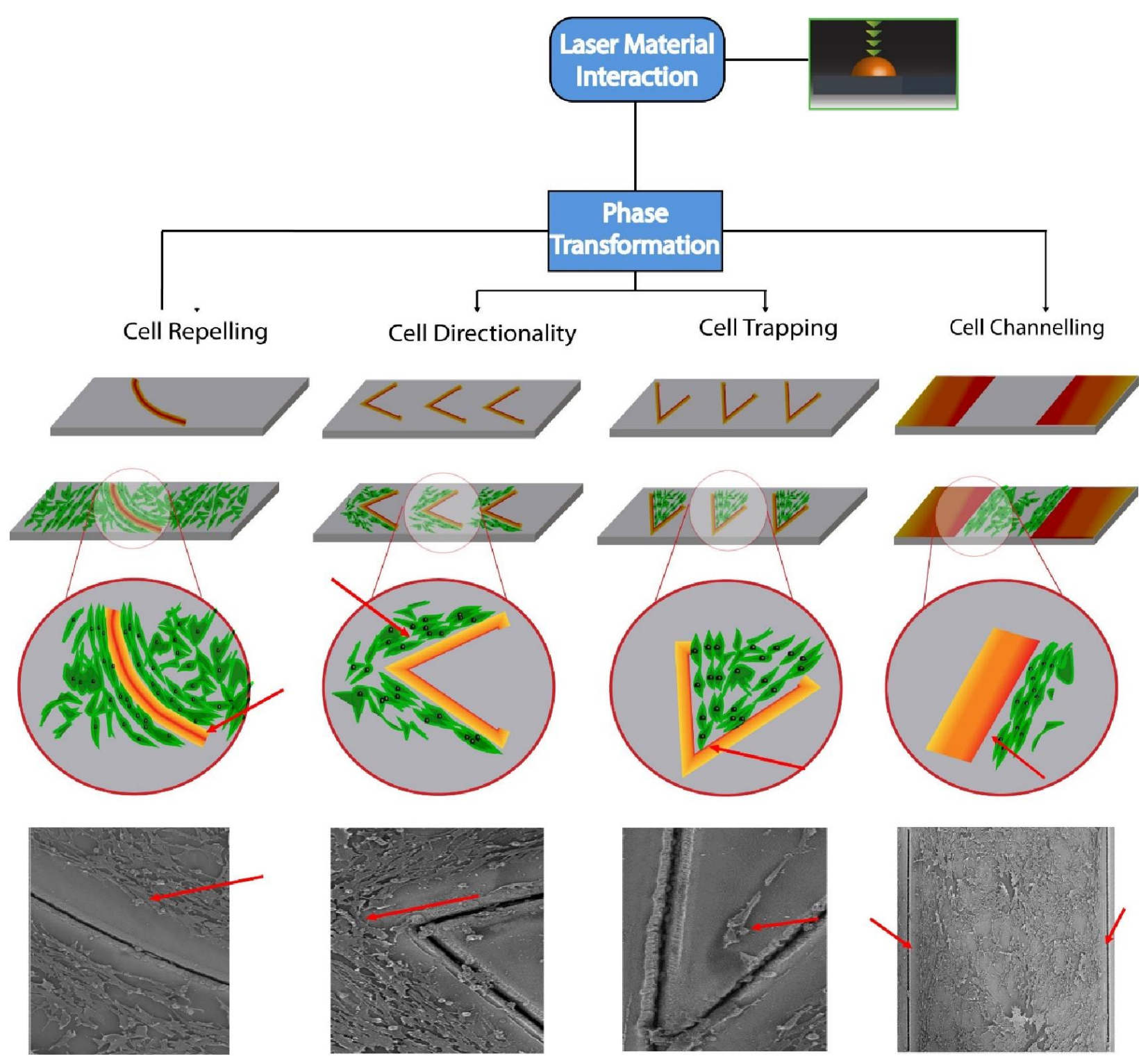


Figure 5-3: Illustration of applications of substrate phase transformation of silicon

The phase transformed zones are studied for their effect on adhesion and migration of fibroblasts. After 24 hours of incubation on these substrates, the samples with cells are fixed and critical point dried. Figure 5-3 suggests healthy growth of cells on bulk silicon. The cells on silicon proliferated and adhered well to the surface. The migration patterns were random and bulk silicon presented no effect on the directionality of cells. In contrast, areas of phase transformation presented different patterns of growth. Cell repelling, directional growth of cells, cell channelling and single cell trapping is observed. Cell repelling is predominantly a property of the primary phase transformed zone.Higher peak power results in a significantly larger primary phase transformed zone. Figure 5-4(A-C) illustrates cell repelling. There is no crossover of cells from either side of the irradiated area, observed conclusively in EDX mapping (Figure 5-6). Moreover, the repellant area's significant effect leads to cells aligning themselves along the repelling zone. Magnified images of this zone further affirm the strength of the cell repellant zone (Figure 5-4 B). It is observed that the side of the cell facing the primary phase transformed zone has significantly lesser filopodia and adhesion points. Tilted images (Figure 5-4C) of the same reveal that the cell in fact curves upwards and is not completely attached to the substrate. The primary phase transformed zone promotes cell repelling and the secondary zone promotes directional growth of cells. Similar to the primary phase transformed zone, the secondary phase transformed zone elicits significant effect on the cells. All the cells in the secondary phase transformed zone grow in the same direction as that of ultrafast femtosecond pulse and silicon interaction. Therefore, the direction of cells can be precisely controlled accordingly. Cells are seen bending with the change in direction (Figure 5-4(D-F)). Depending on peak power the width of the secondary phase transformed zone can be controlled. The entire secondary phase transformed zone consists of unidirectional cells. New cells grow in the same direction as parent cells. This area of unidirectional cells is significantly more on areas synthesized by high peak power as compared to area with lower peak power. The length of the cellis also monitored on its major axis among the unidirectional cells. A 19\% increase in length of cells is seen in secondary cell repellant zones of higher peak power compared to lower peak power. The length of the cells gives an indication of its apparent health [96]. This may also be taken as an indication of healthy adhesion.This difference in properties between the phase transformed zones is owing to formation high temperature phases in the primary phase transformed zone and low temperature 
phases on the secondary phase transformed zone. Cell channelling is another property that is highlighted in this research study (Figure 5-5(A-C)). Combining the properties of the primary and secondary phase transformed zone, the migration of cells in a channel was achieved, a major requirement in lab-on-a-chip and other microfluidic devices. Another major current need in the biomedical industry is to trap single cells. Drug testing, fundamental biological studies and toxicology require studying singular cells. Silicon is already being used exclusively to design such chips. Here it is seen that ultrafast laser pulses in femtosecond regime can generate zones to trap single cells for further analysis. Figure 5-5(D-F) shows trapping single cells. Conjoining two primary phase transformed zones result in areas sufficient only for a single cell to grow resulting in trapping a single cell and keeping other cells away. Not only is this technique able to synthesize these phase transformed zones, it also allows for precise control of width of these areas (Figure 5-6). To further study the cell material interaction fluorescence microscopy was performed by dyeing the actin cytoskeleton and the nucleus of the cells (Figure 5-7). The cells that grow along the secondary phase transformed zone are observed to be oriented along this line. The actin cytoskeleton of these cells are evidently parallel to these zones. Moreover, when cells bend along this zone, their actin cytoskeleton shows a similar trend. Furthermore, the actin cytoskeleton are arranged in a closely packed form in cells that grow in the secondary phase transformed zone compared to the control zone.
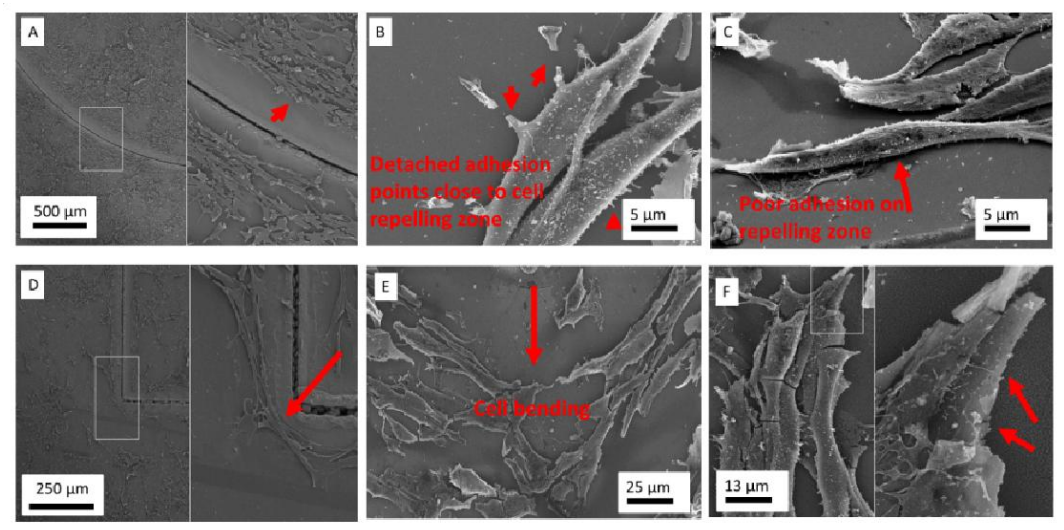

Figure 5-4: (A-C) Cell repelling phase transformed zones. Red arrows at (A) Represent the cell repelling zone (B) Represent broken adhesion points close to repelling zone and several filopodia 
away from zone (C) Represents curved cell growth with poor adhesion. (D-F) Cell directionality. The red arrows at (D-F) represent bending of cells along the zone.
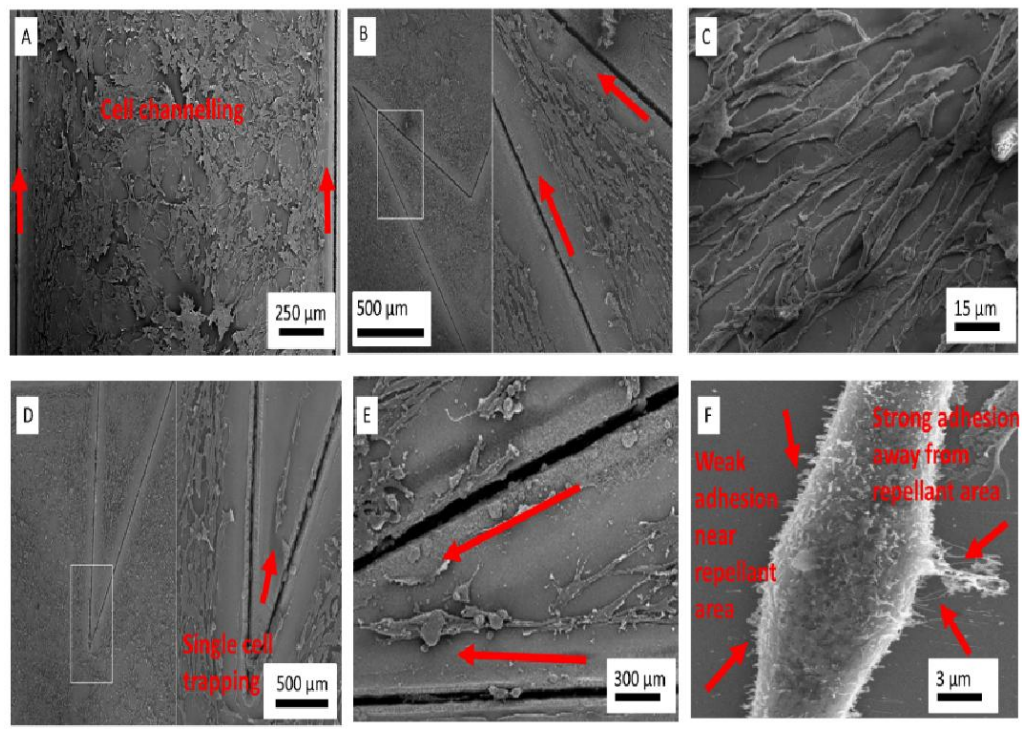

Figure 5-5: (A-C) Cell channeling with in a directional fashion. The red arrows at (A-C) Represent channeling of cells along the zone. (D-F) Single cells trapping combining primary and secondary phase transformed zone.The red arrows at (D-F) Represent trapping of cells (F) Magnified image of filopodia interaction with phase transformed and plain silicon.

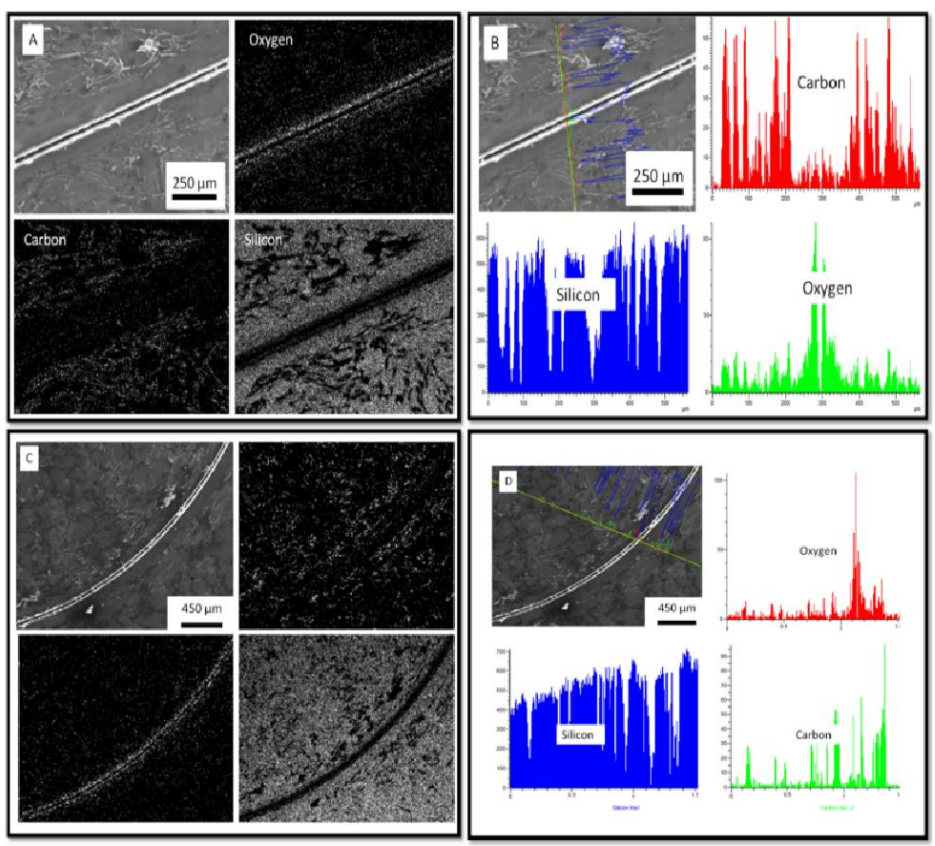


Figure 5-6: EDX micrographs after cell culture at 8.76 MW (A-B) and 1.31MW (C-D). The images indicate cell growth in areas away from primary phase transformed zone.
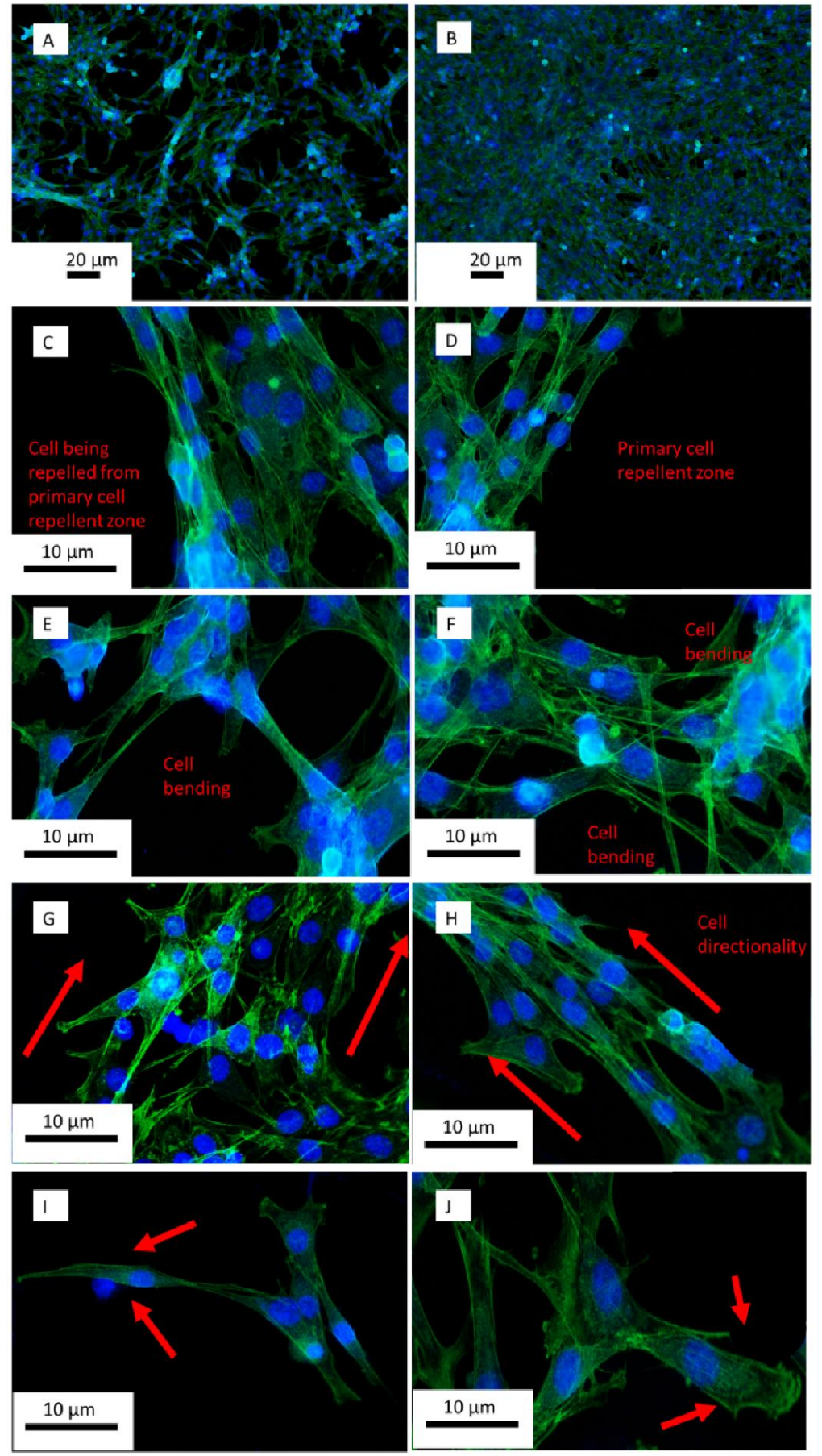

Figure 5-7: Fluorescence microscopy images of actin and nucleus position of cells on primary and secondary phase transformed zones. (A-B) Control/plain silicon (C-D) Cells being repelled 
from primary phase transformed zone and aligning along secondary phase transformed zone (GH) Cell channeling in a single direction (I-J) Single cell isolation.

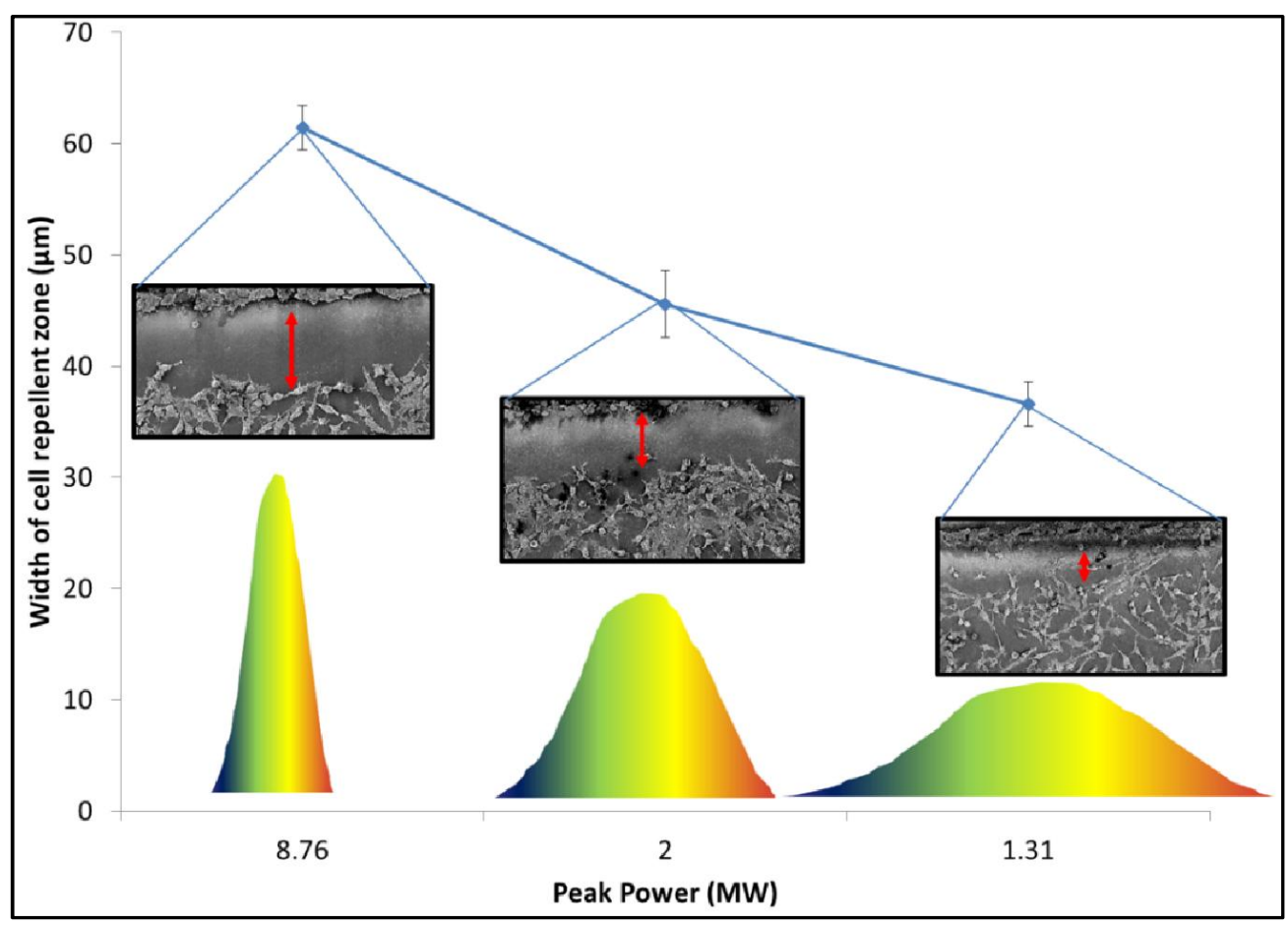

Figure 5-8: Graph illustrating the correlation between pulse duration and width of cell repellent zone.

This shows that the cells are travelling only in one direction as opposed to cells in the control areas that grow randomly. It is observed that the width of the cell repelling zone can be controlled precisely by varying the peak power (Figure 5-8). Cell culture studies show that cells are completely repelled from the areas where a new phase is reported. Properties like cell trapping, directional (bending as well as channeled) cell growth is reported. Cell repelling according to the formation phase transformed zones and its properties is observed. The images show that the cells are not killed but only completely prevented from adhering to the surface. The hydrophobicity of this surface may cause cell repelling. The extracellular molecules promote cell adhesion on surfaces. These molecules are adsorbed from cellular media or from deposits made by the cells. However, in cases where the surface is hydrophobic, these proteins 
are not attached in the proper geometrical form. This in turn leads to poor cell adhesion [140]. The electrical conductivity of the substrate may also play a role in the adhesion of cells even if no electrical stimulation is made. Higher electrical conductivity may result in significantly better cell growth [141]. It is known that silicon has better electrical conductivity compared to its oxides owing to the presence of electrons. Therefore, the cell repellant zones may show controllability based on its conductivity. Furthermore, the thickness of the oxide layer may also influence the electrical conductivity as observed by Scahfft et.al [142]. They stated that thermal conductivity has a direct correlation with the thickness of the oxide layer. Lower pulse durations will result in thinner oxide layers compared to higher pulse durations. Therefore, at $8.76 \mathrm{MW}$ a thin oxide layer is formed corresponding to a decrease in electrical conductivity and hence lower cell growth. In addition to complete repelling a notable process of directional orientation of cells is noticed. In past research studies this was mainly achieved by topographical changes [143, 144]. However, in this study similar results were obtained without the disruption of surface topology. Directionality can be explained by means of the tensegrity theory of cells. The formation of new silicon polymorphs and other changes to the phase of silicon promotes surface heterogeneity and consequently differences in surface energies. The change in surface energy dictates the formation of focal adhesions and localization of vinculin, aligning with the secondary phase transformed zone[145, 146].

\subsection{Summary}

Controlling the growth of cells is crucial in several key research areas such as tissue engineering, MEMS devices and other diagnostic chips. In all these areas silicon is primarily employed. However, the need to re-program silicon from an innately proliferative surface to a cell repelling surface has not been achieved. The fabrication of phase transformed silicon surfaces by ultrafast laser pulses in the femtosecond regime that act as cell repelling surfaces is introduced. Additionally, properties of cell trapping, cell channelling and directional cell growth is also observed.The phase transformed zones consist of silicon polymorphs and altered surface characteristics which have been analyzed in this study. Cell growth can be precisely controlled based on peak power of the ultrafast pulses. SEM images show $100 \%$ cell repelling in a precise fashion. This unique and novel research study paves the way for application of this technology in a multitude of areas including but not limited to diagnostic devices, tissue engineering constructs, biosensing apparatus and perhaps anti fouling technologies. 


\section{Chapter 6}

\section{Cancer cell interaction studies withfunctionalized phase transformed silicon}

In addition to repelling of fibroblasts, the interaction of cancer cells with the repelling silicon is studied. The programming of silicon from biocompatible to functionalized silicon that is capable of preferentially selecting cell populations for survival and/or death is introducedSilicon is extensively employed in cancer diagnostics as tumor-chip devices. However, it necessitates surface manipulation either by addition of targets or changes to its topography. The degree of functionalization is programmed to dictate the degree of repulsion of cancer cells. Silicon is functionalized by the interaction of ultrafast laser pulses with a separation time of micro to nanoseconds causing a temperature gradient that results in rare phases of $\mathrm{SiO}_{2}$ in conjunction with differing silicon crystal lattices along the interaction zone. Unstable phases of $\mathrm{SiO}_{2}$ are synthesized during functionalization and remain stable at ambient conditions. This change in phase of silicon as well as its oxides contributes to changes in surface chemistry as well as surface energy. Consequently, cancer cells respond resulting in precise control of migration, cytoskeleton shape, direction and population of cancer cells. To the best of our knowledge, functionalized silicon without changes in morphology or any additive layers has not been reported before. The versatility of this technique enhances opportunities in an array of applications. This unique programmable functionalized silicon has the potential to change current trends in cancer research and generate focus on biomaterials as cancer repelling or potentially killing surfaces.

\subsection{Introduction}

Research in cancer treatment is geared towards targeted therapy, gene therapy, radiation therapy, photodynamic therapy and biological therapies [147-151]. While the use of biomaterials for cancer therapies is envisioned, its usage has been limited as drug carriers. The use of functionalized biomaterials as surfaces that can potentially be enforced to prevent cancer regression is foreseen. Likewise, the detection of cancer is also receiving much attention. Research in cancer detection primarily focuses on the use of microfluidic channels to detect the 
presence of cancer, often using proteins specific to the cancer cells[152, 153]. Therefore, the use of a functionalized material that is capable of promoting the directional flow of cells as well as trapping singular cells for analysis is sought after. Thusly novel programmable functionalized silicon that is capable of deactivating the growth and spreading of cancer while concurrently stimulating directional growth as well as presenting a single cell trapping surface is introduced.

Biomaterials in cancer treatments arouse predominantly in the area of imaging, drug delivery and targeted therapy. Purushotham et al. implemented magnetite coated polymer core shell materials to release anti-cancer drugs [154]. This core shell material is temperature dependent and is therefore able to selectively release the drug over time. Several such nanoparticles are being investigated as possible candidates for drug delivery systems. However, the toxicity of the nanoparticles itself is a concern. For instance, Singh et al. performed an in-depth review of the toxic effects of iron oxide nanoparticles [155]. It is observed that size as well as concentration has an important role in cytotoxicity. Analogously, cancerimaging also implements similar magnetic nanoparticles for guided imaging[156].Targeted delivery uses nanoparticles as their primary carriers coated with specific antibodies to target precise moieties. Bae et al. studied the applicability of manganese oxide nanoparticles in targeted siRNA (small interfering RNA) delivery as well as magnetic resonance imaging[157].Silicon is also being considered increasingly for cancer therapies. Silicon is used in various forms-crystalline, amorphous, nanoparticles and nanowires [158-161]. Silicon is a viable candidate owing to its biocompatibility and biodegradability. The predominance of silicon in cancer therapies has been limited to nano and micro particles. It is apparent that bulk silicon has not been investigated for its potential anti-cancer properties.

Cancer treatment therapies have focussed on nano and micro forms of silicon while cancer diagnostics essentially focuses on bulk silicon as a base. Diagnostic devices often require significant changes to their surface to suit applications. To this end, researchers rely on additional techniques to change the properties of bulk silicon. Such techniques can be categorized broadly as chemical and topographical techniques [123]. These techniques either change the surface topology of silicon or change its chemical characteristics. Moreover, to change material properties, these techniques occasionally require the inclusion of several additional layers. Modifications to the surface of silicon are required to allow the precisely 
controlled growth of cells. Characteristics such as cell adhesion, direction and single cell isolation necessitate modifications. These properties are specifically of prime importance in micro-fluidic and diagnostic devices. For example, Chen et al. reviewed topography modifications to capture cancer cells [162].Micro pillars were fabricated on the surface to enhance the interactions of cells with the underlying substrate. Such topographical modifications are not only expensive but also limited in their feature size. With respect to modifications based on chemical changes, the introduction of unknown toxins in a biological environment is a possibility. Consequently, there is need for a technique that negates the use of chemicals as well as prevents changes to the topography.

In this chapter, phase transformation of silicon, converting it from a biocompatible surface to a functionalized cancer controlling surface is introduced. Ultrafast laser pulses with nano to microsecond pulse to pulse separation time interact with silicon, modifying its surface without affecting topography. This interaction can be precisely manipulated to induce the synthesis of novel functionalized cell controlling zones by creating changes in surface chemistry. The controlling zones can direct and channel cell migration as well as promote single cell channelling and isolation. Further, the functionalized surface can completely repel cells from its surface via formation of oxide as well as varied crystal structures (Figure 6-1). These unique properties are attributed to the modified silicon surface. Material characterization demonstrates the presence of polymorphs of silicon dioxides such as tridymite, coesite and stishovite. The involvement of high temperature and pressure in the formation of these polymorphs allows for the existence of conventionally unstable phases at ambient temperatures. Further, the high temperature and pressure also induces changes in the crystal lattice of silicon, resulting in the generation of different phases of silicon. In this chapter the synthesis and characterization of this functionalized biomaterial is elucidated. Moreover, the influence of altered surface chemistry on the growth patterns of cancer cells is explored in-depth. 
Versatile- Selective synthesis of functionalized phase transformed zones on any area with any shape or size
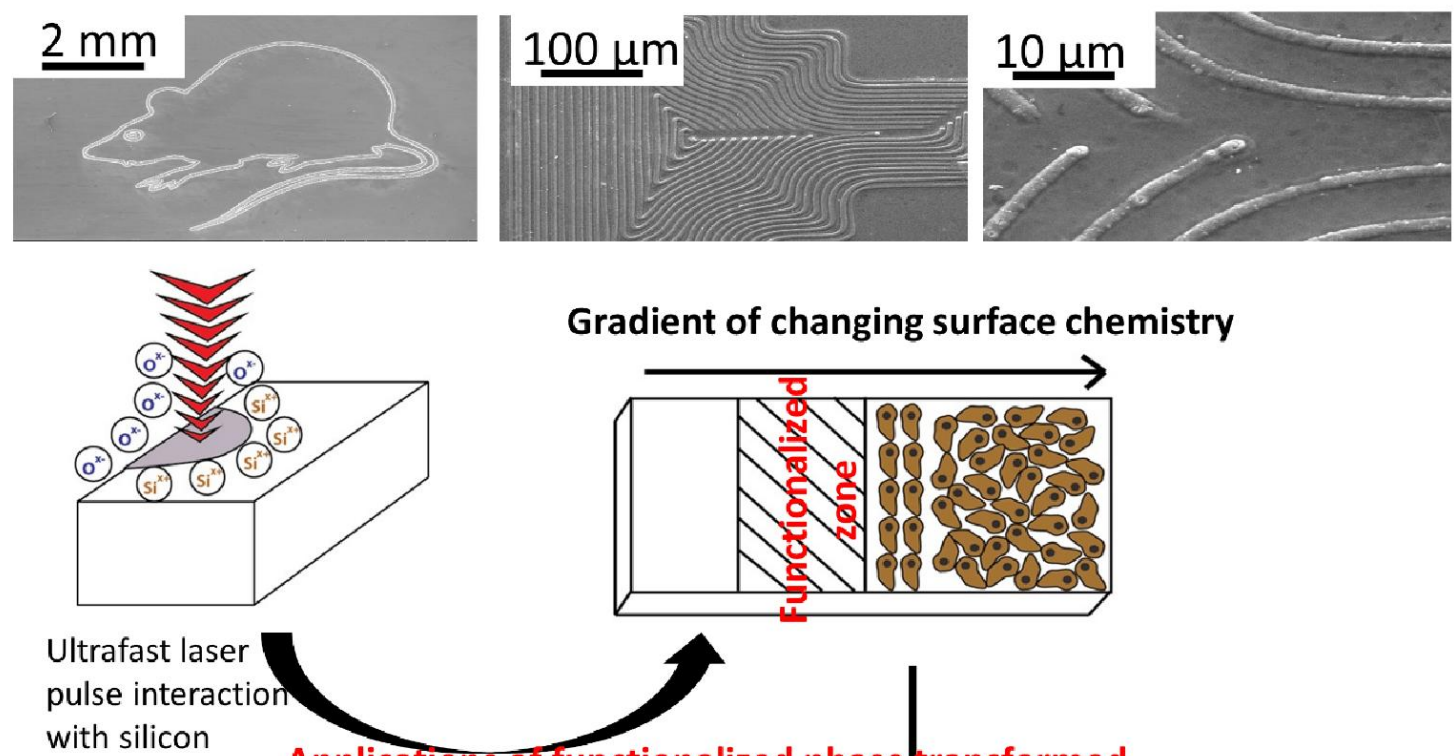

\section{Gradient of changing surface chemistry}

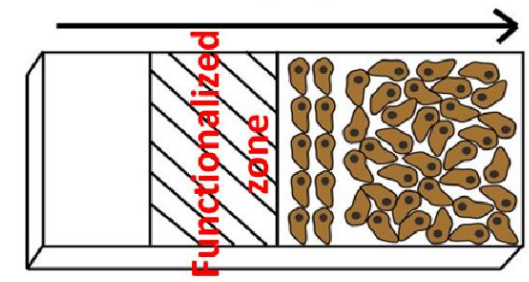
pulse interaction with silicon
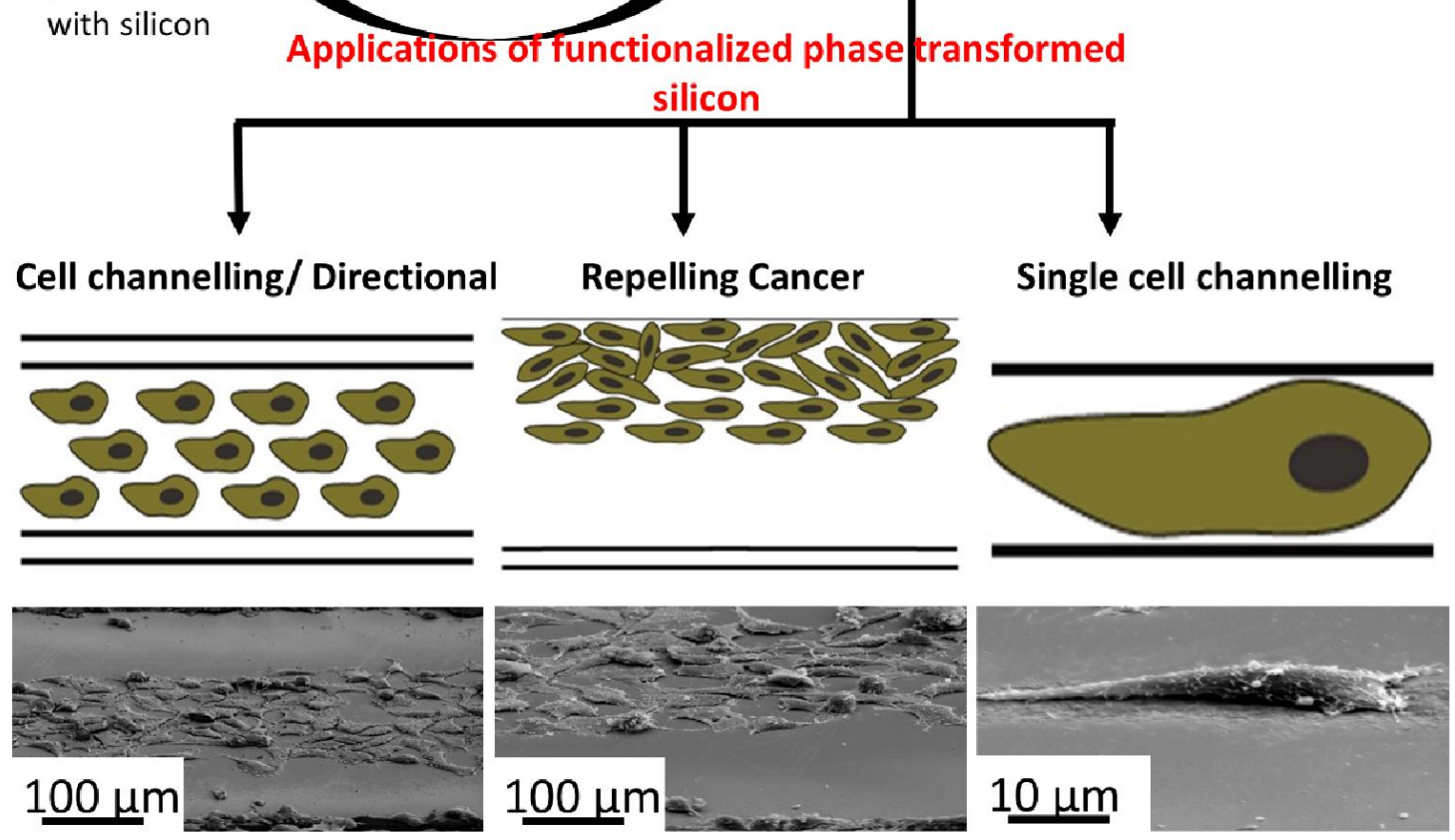

Figure 6-1: Synthesis and application of programmable functionalizedsilicon surfaces. The SEM images of various shapes present the versatility of the ultrafast pulse laser synthesis method. The figure depicts an overall mechanism of fabrication and application of phase transformed functionalized silicon in various areas. 


\subsection{Experimental Detail}

\subsubsection{Synthesis of functionalized zones}

These samples are irradiated using a diode pumped, Yb-doped femtosecond laser system (ClarkMXR Inc. IM-PULSE Series Ultrashort Pulse laser) at 4 (low frequency), 8 (mid-frequency) and $26 \mathrm{MHz}$ (high frequency). The wavelength of the laser is $1040 \mathrm{~nm}$. The laser irradiates the samples in an array of lines at distances between $100 \mu \mathrm{m}$ to $2 \mathrm{~mm}$. Further, different shapes to simulate tissue engineering and diagnostic device environments are synthesized. The speed at which the laser irradiates and the power of the laser is maintained at $10 \mathrm{~mm} / \mathrm{s}$ and $15 \mathrm{~W}$ respectively. The laser pulse width or peak power plays an important role and is varied between 214fs (short pulse), 714fs (mid pulse) and 1428fs (long pulse) corresponding to $8.76 \mathrm{MW}, 2 \mathrm{MW}$ and $1.31 \mathrm{MW}$ respectively. All the parameters are controlled by a computer to facilitate precision and accuracy. In this paper, $4 \mathrm{MHz}, 8 \mathrm{MHz}$ and $26 \mathrm{MHz}$ will be denoted as low, mid and high frequency respectively. With respect to pulse width/duration, 214fs, $714 \mathrm{fs}$ and $1428 \mathrm{fs}$ will be denoted as short, mid and long pulse respectively. All samples after fabrication are ultrasonicated to remove any potential debris before cell culture is performed.

\subsubsection{Understanding the functionalized zones}

Scanning electron microscopy (SEM) (Hitachi S 5200) is performed to characterize the morphology of thefunctionalized silicon surfaces. The samples are loaded onto aluminum stands and gold coated for SEM. Following SEM, Energy-dispersive X-ray spectroscopy (EDX) is carried out to determine the elemental composition of irradiated silicon. Micro-raman spectroscopy as well as X-Ray photon spectroscopy (Thermo scientific K-alpha) is used further to study surface composition quantitatively and qualitatively. X-ray diffraction(Siemens D5000 conventional theta/2theta diffractometer) is used as a secondary tool to confirm elemental composition.

\subsubsection{Cancer- functionalized zone interaction}

Cervical cancer cells (HeLa, ATCC, USA) are employed to qualitatively and quantitatively study cancer controllability. The cells are grown in DMEM/F12 medium supplemented with $10 \%$ fetal bovine serum and $1 \%$ pen-strep. The functionalized surfaces are washed with alcohol and DI water and kept under UV light for 20 minutes. Subsequently, the substrates are placed in petri 
dishes and HeLa cells are seeded at a density of $10^{5}$ cells $/ \mathrm{ml}$, totalling $3 \mathrm{ml}$ volume per dish. The petri dishes are placed in an incubator for 24 and 48 hours.

For SEM imaging, after the incubation period the samples are fixed in $2 \%$ glutaraldehyde in $0.1 \mathrm{M}$ sodium cacodylate buffer $\mathrm{pH} 7.3$ for an hour. Next, the samples are immersed in $0.1 \mathrm{M}$ sodium cacodylate buffer with $0.2 \mathrm{M}$ sucrose at $\mathrm{pH} 7.3$ for 20 minutes. Dehydration in increasing concentrations of alcohol for 20 minutes each is followed. The samples are then critical point dried. All imaging was conducted at $5 \mathrm{kV}$ and magnification was varied between 100 and 10,000 times.

For fluorescence microscopy the samples are first fixed in methanol free paraformaldehyde followed by incubation with milk to prevent non-specific binding. To stain the actin cytoskeleton, the samples are incubated with Alexa fluor 488(Life Technologies) followed by DAPI(Life Technologies) to stain the nucleus. The samples are studied using a fluorescence microscope (Nikon).

\subsubsection{Statistics}

All experiments are carried out in triplicates and the data points are averages unless otherwise mentioned. The error bars indicate standard deviations.

\subsection{Results and Discussion}

\subsubsection{Synthesis of functionalized zones}

The interaction of the ultrafast pulses with micro to nano pulse separation timeand silicon resulted in functionalized zones due to phase transformations. These phase transformed zones consist of different silicon and silicon oxide polymorphs as well as changes to their crystal lattice. When the pulses interact with the surface of silicon, energy is transferred to the silicon surface. Due to the ultrashort nature of the pulses, multiphoton excitation processes occur. The electron temperature is now higher than the lattice temperature resulting in electron thermalization[163]. These electrons cool down and release phonons [164]. At this point the temperature of laser-material temperature evolves. It should be noted that the frequency of the pulses is an important consideration. When the required energy has accumulated, silicon converts from its solid to liquid state.Ablation in the femtosecond regime generates recoil pressure[165]. These recoil pressures are in turn responsible for phase changes in the lattice of silicon. 
In this research study, the peak power of the pulse is varied based on the frequency as well as duration of the pulses. The frequency and the width of the pulse are inversely proportional to the peak power. The surface temperature at silicon is calculated as[132]

$T_{n}=2 \alpha \frac{\left[1-\left(\frac{2}{3}\right) \alpha\right]}{\left(1+\alpha^{2}\right)} \frac{T_{m}}{(1-\alpha)}\left[1+\frac{\alpha^{n}-\alpha}{n(1-\alpha)}\right](6.1)$

Where $T=\sqrt{\frac{2 a}{\pi^{3} t_{p}}} \frac{4 K(1-R) P}{k f d^{2}}(5.1)$

$t_{p}$ is the pulse duration, $a$ is the thermal diffusion coefficient, $\mathrm{k}$ is the heat conduction coefficient, $\mathrm{R}$ is the reflection coefficient, $\mathrm{K}$ is the residual energy coefficient, $\mathrm{d}$ is the laser spot diameter and $\alpha$ is a ratio of pulse duration to pulse interval.

Table 1 (Supplementary information) presents the temperature generated on the surface after multiple femtosecond pulses interact with the surface of silicon. It is evident from the temperatures that the frequency of the pulses plays a more dominant role in changing phases compared to the duration of pulses. However, the width of the functionalized phase transformed zone is determined by the duration of the pulses. Thusly, the frequency of pulses determines the presence or absence of the functionalized zone and the pulse duration determines the width of the functionalized zone.

It is seen that only at low frequency, a functionalized zone is being generated. At mid frequency and high frequency, a functionalized zone is not generated but the phase of the substrate is changed inducing varying cell growth characteristics which will be delineated below. To sum up, a minimum temperature of approximately $6200 \mathrm{~K}$ is required to generate this phase transformed zone. In spite of the similar temperatures being generated at the surface at short, mid and long pulses, the laser-material interactions are different owing to varied heat dissipation mechanisms. Changes in phases are not solely attributed to temperatures but changes in pressure generated during laser-material interaction as well. During ablation, the pressure being generated is proportional to material ablation. The formation of a trench reflects light from the sides which in turn increases the rate of ablation [134]. Subsequently, magnitude of pressure waves being generated increases. Higher pulse durations will contribute to the formation of a melt layer in this trench [166]. This may result lesser magnitudes of pressure waves. The difference in pressure 
waves suggests the formation of different phases of silicon as well different widths of the phase transformed zones.

\begin{tabular}{|c|c|c|c|}
\hline $\begin{array}{l}\text { Pulse Duration } \\
\text { (fs) }\end{array}$ & $\begin{array}{l}\text { Frequency of pulses } \\
(\mathrm{MHz})\end{array}$ & $\begin{array}{ll}\text { Peak } & \text { Power } \\
(\mathrm{MW}) & \end{array}$ & $\begin{array}{l}\text { Surface temperature after } \\
\text { multiple pulses }(\mathrm{K})\end{array}$ \\
\hline 214 & 4 & 17.52 & 6242.50 \\
\hline 214 & 8 & 8.76 & 4414.70 \\
\hline 214 & 26 & 2.70 & 2449.70 \\
\hline 714 & 4 & 5.25 & 6244.10 \\
\hline 714 & 8 & 2.63 & 4416.20 \\
\hline 714 & 26 & 0.81 & 2451.20 \\
\hline 1428 & 4 & 2.63 & 6245.50 \\
\hline 1428 & 8 & 1.31 & 4417.70 \\
\hline 1428 & 26 & 0.40 & 2452.70 \\
\hline
\end{tabular}

Table 1: Surface temperature on silicon after multiple pulse interaction at various peak powers.

\subsubsection{Material investigation of functionalized zones}

Functionalized phase transformed zones on silicon are observed using a scanning electron microscope (SEM) in conjunction with Energy-dispersive X-ray spectroscopy (EDX). SEM images show the presence of trenches followed by areas of phase transformation (observed by minor change in color). EDX reveals the presence of oxides on these phase transformed zones. This ratio of silicon to oxygen on the phase transformed surfaces does not directly relate to peak power. Both the duration of the pulses as well as the frequency of the pulses have an effect on oxide content. With respect to frequency of pulses, a lower frequency causes greater oxide content in the phase transformed zones (Figure 6-2(A, C)). As the frequency of pulses increases, it is evident that the oxide content at both pulse durations decreases. Moreover, oxide content at higher frequencies for both pulse widths are essentially the same. These results confirm the dominant role of frequency of pulses. The role of these oxides are further probed to study a potential correlation to cancer repelling properties. 
To study the oxides on the functionalized phase transformed zone, micro-Raman analysis is performed (Figure 2B). The peak for silicon and native oxide layer dominate the graphs due to substrate effects. There are several small peaks across the Raman spectrum. These peaks are indicative of silicon oxides of different crystal orientations. Micro-Raman spectra show presence of coesite, tridymite and stishovite[167-169]. Coesite and stishovite are high temperature phases of silicon while tridymite is a low temperature phase. Coesite and stishovite are not stable at ambient temperatures but transition into other polymorphs which includes changes to its lattice parameters and hence they become metastable at normal conditions. A broader peak for tridymite might point to presence of a stable silicon polymorph compared to other oxides. Each of the peaks in the micro-Raman graph also differ in their width. This is indicative of the presence of crystalline and amorphous phases. It is observed that at shorter pulse width, the crystalline peak of silicon is wider, pointing to the presence of amorphous silicon. Therefore, it is seen that at shorter pulse widths, high temperature phases are formed. Further, shorter pulse widths also promote generation of amorphous silicon.

To further quantify functionalized phase transformed zones, micro-Raman, X-ray diffraction and $\mathrm{X}$-ray photoelectron spectroscopy is performed. Figure 6-2(D) illustrates XRD micrographs that confirm the presence of different crystal structures in the functionalized phase transformed zones namely (111), (220) and (311). Ultrafast laser transition induces nucleation sites. This is followed by melting and subsequent crystallization in various orientations [170]. It is observed that the (111) peaks have higher intensity at long pulse compared to short pulse. This is due to highly arranged stacked nature of the (111) crystals at long pulses. Furthermore, the dominance of the (111) peak is due to its lower surface energy. It is evident that the curves show an upward trend at lower angles. These upward trends correspond to amorphous phases on the substrate. The minor shifts in peaks are attributed to instrument error. XPS micro-graphs illustrate the presence of different silicon and silicon oxides (Figure 6-2E). A shift in peaks is detected around $103 \mathrm{eV}$ and $99 \mathrm{eV}$. It is known that higher binding energy correlates to higher positive energy of the atoms. Further, the arrangement of the bonds and the ring size also leads to a shift change. Thusly, a small ring size due to smaller O-Si-O bond angles causes increase in shifts[171]. 

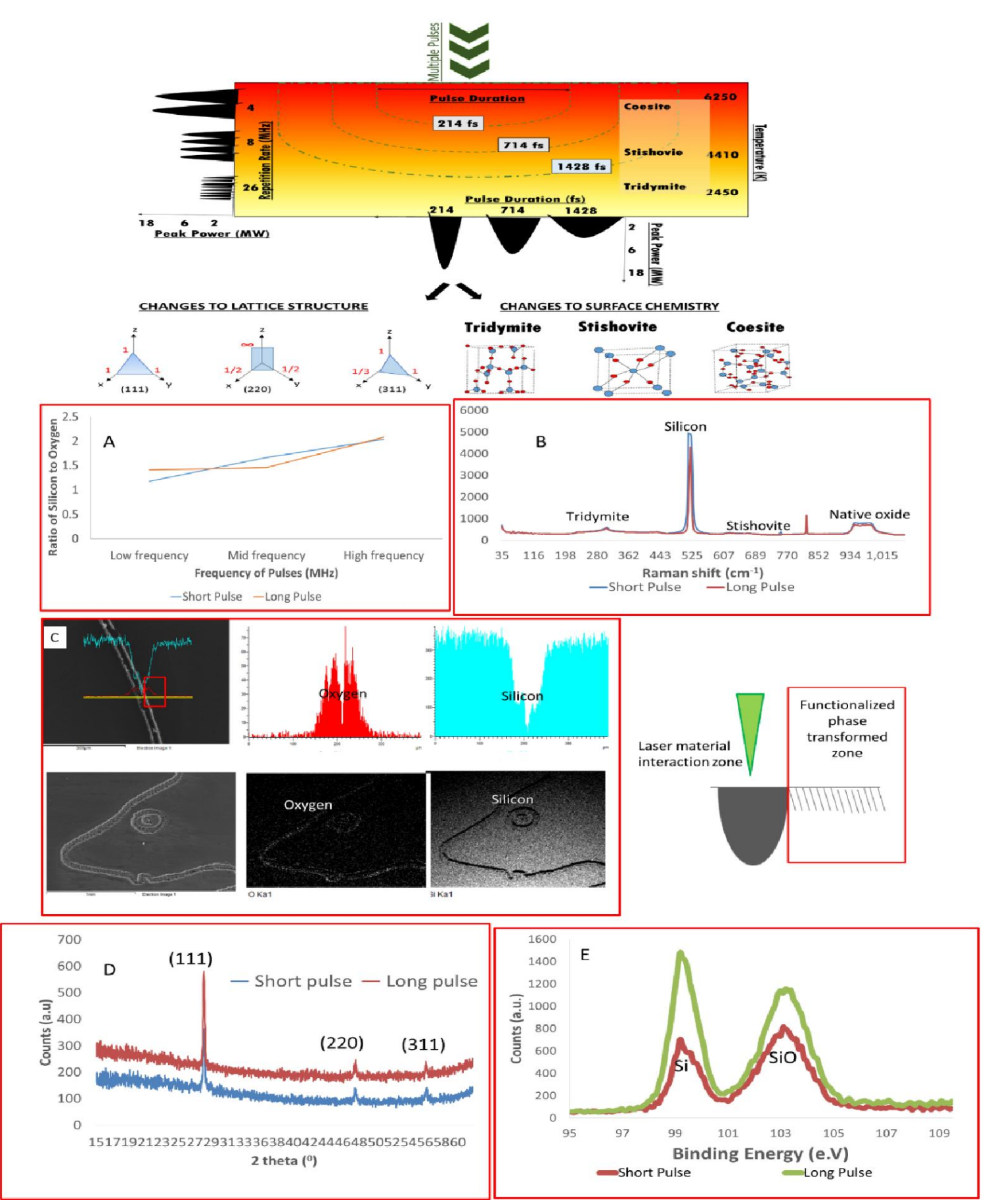

Figure 6-2: Illustration of pulse-silicon interaction and causative changes in silicon (A) Ratio of silicon to oxygen at different pulse widths (B) micro-Raman spectra of functionalized phase transformed silicon at different pulse widths (C) EDX in mapping mode reflecting oxygen and silicon content on functionalized zone (D) XRD micro spectra and (E) XPS analysis of functionalized phase transformed silicon. 


\subsubsection{Cancer cell-functionalized functionalized zone interaction}

Subsequent to material characterization, the growth, proliferation and adhesion characteristics of cervical cancer cells is studied on functionalized zones. The formation of the functionalized zones is adjacent to grooves irradiated by the laser. The areas further away from the grooves are left relatively unchanged. Further, a gradient of material change is observed away from the irradiation zone. This change in material characteristic influences the growth properties of cells.

Studies were performed at two time points, 24 and 48 hours. HeLa cells cultured on the surface showed interesting adhesion and growth characteristics after each time period. By varying pulsematerial interaction, the cell growth was controlled. Figure 6-3A illustrates growth over a 24 hour period. The HeLa cells at 24 hours grow randomly and the number of cells on substrates with different parameters are similar. At short pulses, the number of cells are than at mid or long pulses. On average, a change in frequency of pulses did not influence the number of cells at 24 hours. However, the orientation of cells varied depending on the growth area.

At high frequency of pulses, cells are observed all over the substrate, on functionalized phase transformed zones as well as bulk silicon. Cells are randomly arranged and well spread out. Towards the groove however, cells stop abruptly with the majority of cells aligning themselves along the groove. At low frequency, the cells are seen growing along the line, potentially adhering well due to melt zone. At mid frequency, the cell characteristics are in between those of low and high frequency. There is no abrupt stopping at grooves and cells seem to grow over grooves. At 48 hours (Figure 6-3(B)), the HeLa cells showed markedly different growth characteristics compared to the cells at 24 hours. As evidenced in Figure 6-3 the cell numbers as well as cell orientation and growth properties are significantly different with respect to pulse duration as well as frequency of pulses. 

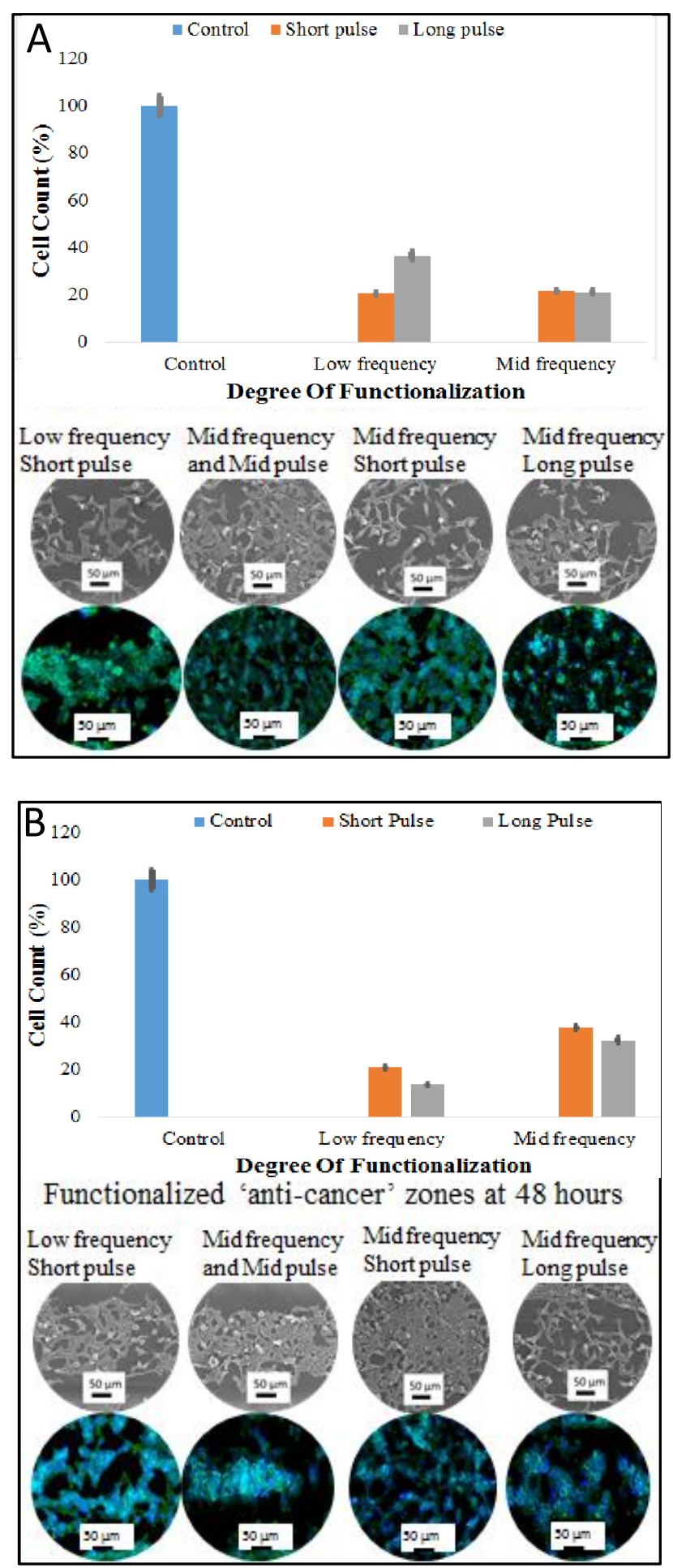

Figure 6-3: Cell-functionalized material interaction after seeding for (A) 24 hours and (B) 48 hours. Scanning electron microscopy as well as fluorescence microscopy images showing cell growth and repelling at each functionalization parameter is presented. 

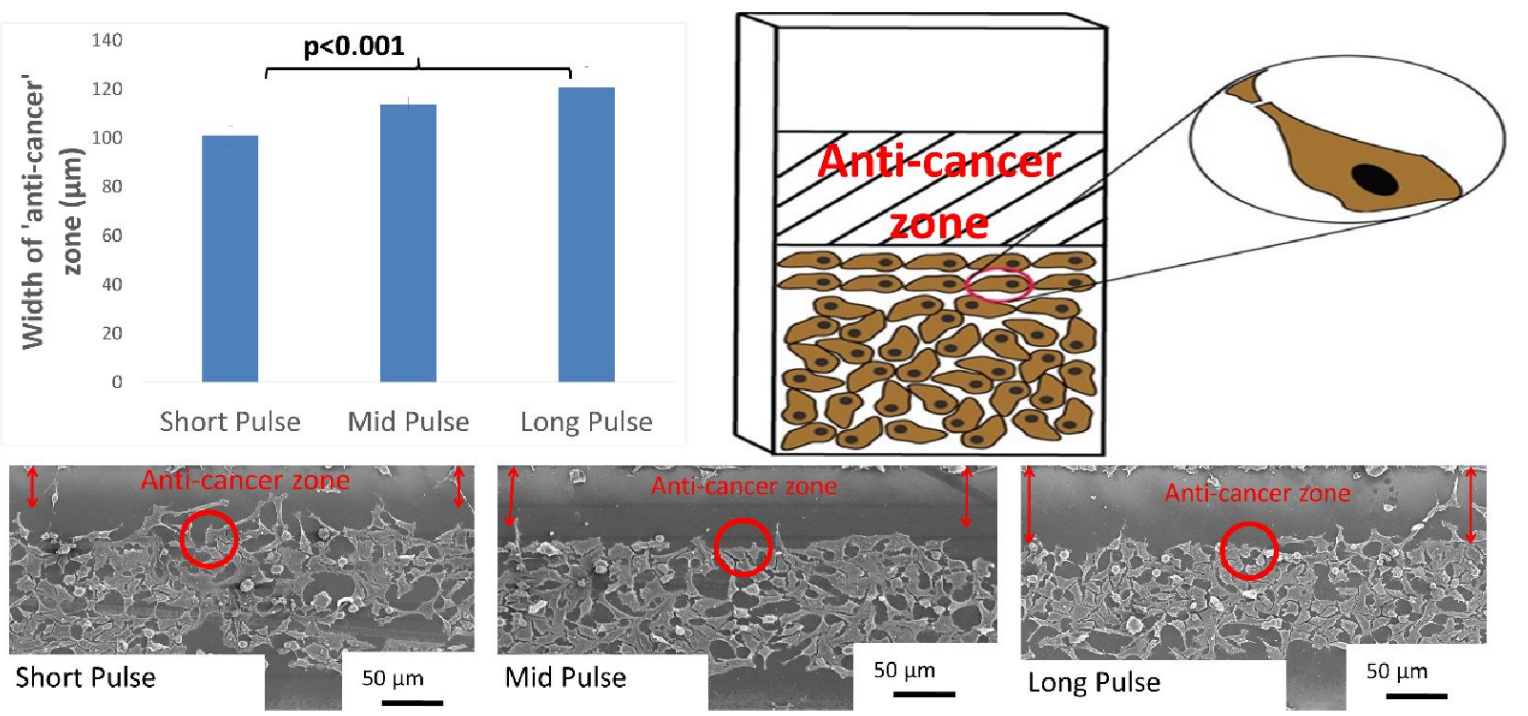

Mid Pulse

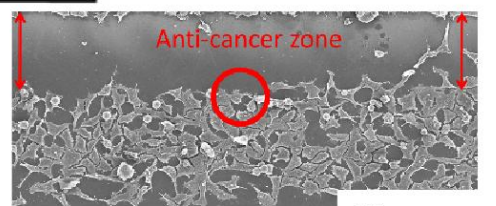

Long Pulse

$50 \mu \mathrm{m}$
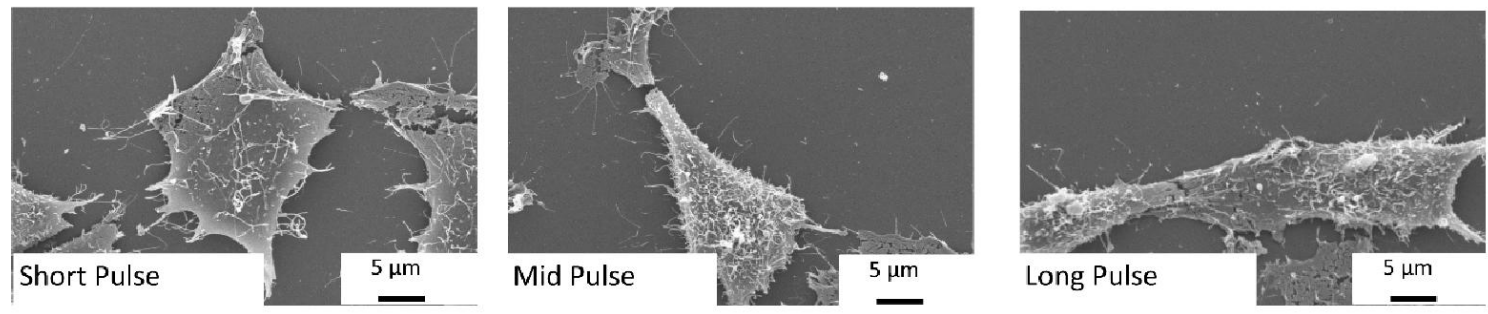

Figure 6-4: Width of functionalized zone with increase in duration of pulses. The SEM images are representative images at different pulse widths and the red arrows indicate functionalized phase transformed zones.

Low frequency consistently generated wide functionalized zones at all pulse durations. At mid frequency, the cells further away from the grooves were flattened and grew randomly. The majority of cells along the grooves grew oriented to the grooves. Compared to other frequencies, it is clear that at mid frequency cells reproduced at a higher rate. The presence of a significantly higher number of rounded cells are indicative of higher cell division rates [172]. At high frequency, the number of cells that orient themselves along the groove are less. Further, cells growing away from the grooves do not attach and spread well on the surface. While frequency 
determined presence or absence of functionalized zone, the pulse durations determined the width of these functionalized zones (Figure 6-4).

The most interesting phenomenon is the apparent activation of the functionalized zone at 48 hours of incubation. Comparing Figure 6-3(A) with Figure 3 (B) at low frequency, it is evident that cells have moved away from the functionalized zone. Furthermore, the cells next to the functionalized zones orient themselves along the outer areas of the functionalized zone. This phenomenon could be attributed to the migration of cells from one area to the other owing to changes in mechanical and chemical characteristics of the functionalized zone. Figure 6-5 illustrates filopodial formation and protrusion away from functionalized zones as opposed to random growth on control silicon surfaces. When the ultrafast pulses interact with the silicon surface, a groove and the functionalized zones are generated. The heat generated during interaction dissipates laterally along the surface creating a gradient on the surface of silicon. This results in different material properties throughout this gradient. Owing to this unique feature, a trend is observed with HeLa cells after 24 and 48 hours of cell seeding.

At 24 hours even though the majority of cells accumulate away from the functionalized zones, there are certain cells that tend to grow on these zones. However, at 48 hours, a clear migration of cells is observed. The HeLa cells that initially attached to the functionalized zones migrate away from these zones in both directions. The unique behaviour of cells is attributed to haptotaxis[173]. Haptotaxis is movement of cells with respect to an adhesion gradient. These functionalized zones create a gradient of less adhesive substrates thusly enabling the haptotaxis of cells.

The cell adhesion and proliferation characteristics are further studied using fluorescence microscopy. The actin cytoskeleton and the nucleus of the cells are stained. Figure 6 illustrates results from fluorescence microscopy after 24 and 48 hours. Fluorescence microscopy confirmed the results obtained from SEM. Further, it also outlined the adhesion characteristics on plain silicon (Figure 6-6 (A-C)) and on the functionalized zones (Figure 6-6(D-F)). It is observed that the cells grow in a random fashion in no particular direction on control silicon. 

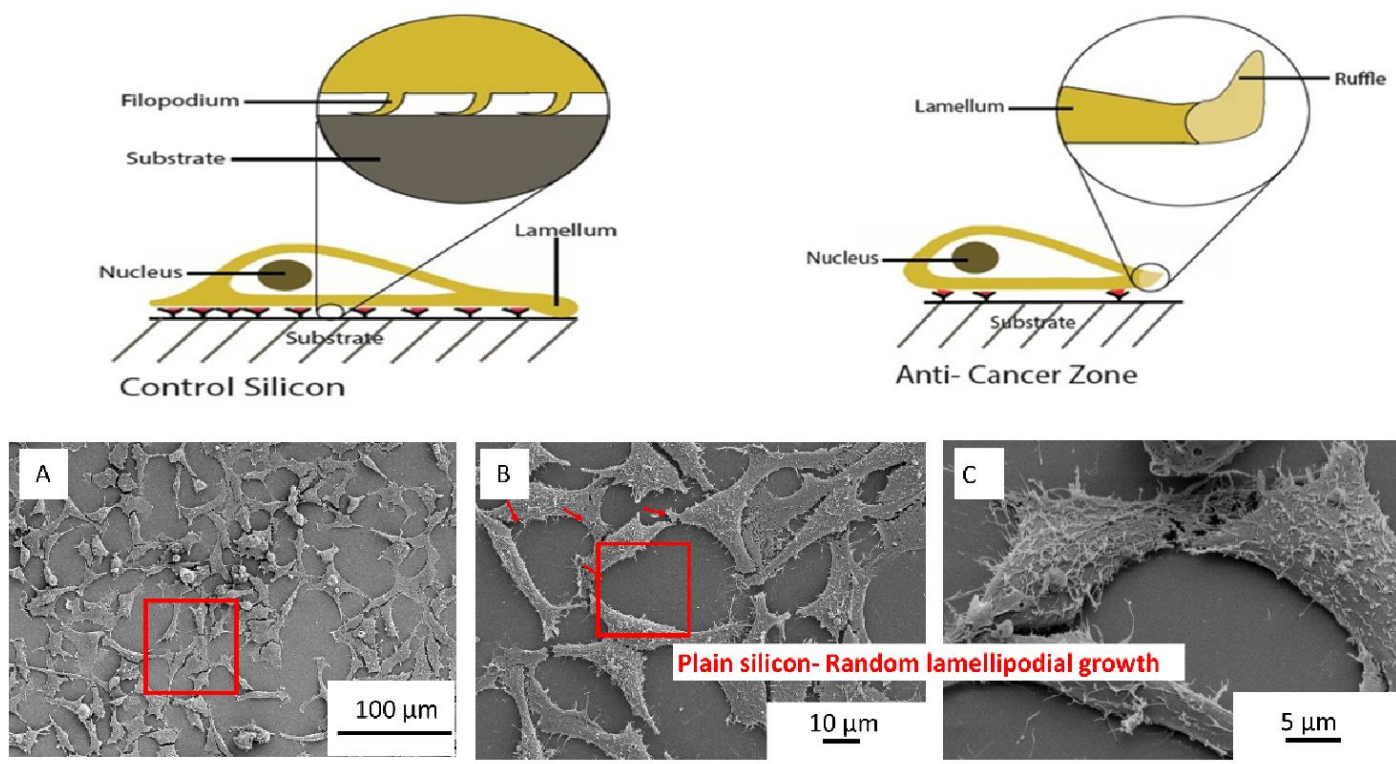

Anti- Cancer Zone
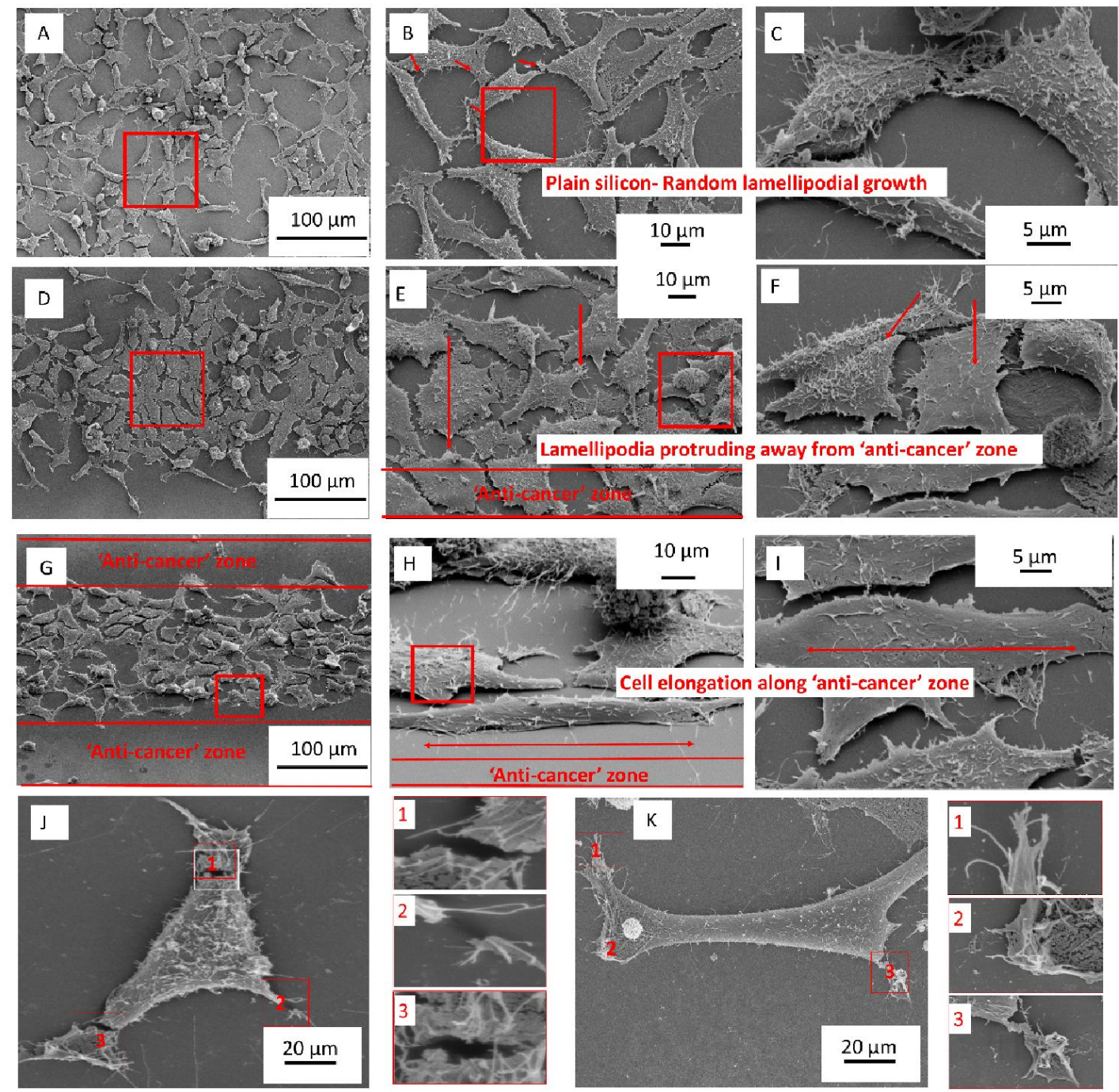

Figure 6-5: Analysis of lamellipodial and filopodial growth on control silicon as well as

functionalized phase transformed silicon. (A-C) HeLa cells cultured on control silicon (D-E)

Hela cells cultured near functionalized zones and properties of lamellipodia on such zones (G-I)

HeLa cell elongation along functionalized zone $(\mathrm{J}-\mathrm{K})$ The breaking up of lamellipodia close to functionalized zones. 
The focal adhesion points are also randomly observed. Focal adhesions are points that interact with the substrate and aid in adhesion and migration (Figure 6-6(G)).Furthermore, the formation of stress fibers is also observed on cells (Figure 6-6 (H)). Stress fibers convert the mechanical signals from the substrate into cues for the cell [174].The intensity and organization of stress fibers differs depending on the location of the cell. For instance, the cells growing away from the functionalized zones consist of stress fibers that are not aligned in any particular direction. However, the cells growing close to the functionalized zones have predominantly aligned stress fibers. Moreover, the growth of cells in areas fabricated at mid or high frequency of pulses have similarly aligned stress fibers. The stress fibers are aligned primarily along the direction of laser material interaction. Stress fibers in cells are also aligned in a direction away from the functionalized zones. In all cells there is the presence of dorsal and ventral stress fibers. It has been observed that the dorsal stress fibers end in focal adhesions and this may be an indication of the leading edge of the cell. In addition to the stress fibers, the nucleus shape and polarization is also studied. Fluorescence images indicate that cells that grown on plain silicon without any substrate modifications have a well-rounded nucleus. On the other hand, the cells on or close to the functionalized zones seem elliptical and polarized. This may be due to the passive and active forces of the actin cytoskeleton[175]. Furthermore, the filopodial extensions are seen clearly protruding in a direction away from the functionalized zones.

From these results, it is evident that the formation of the functionalized zones induces a significant change in material properties which in turn induces changes in the cell-functionalized zone interaction. Two important interaction characteristics will be further discussed in detail in the following section. 

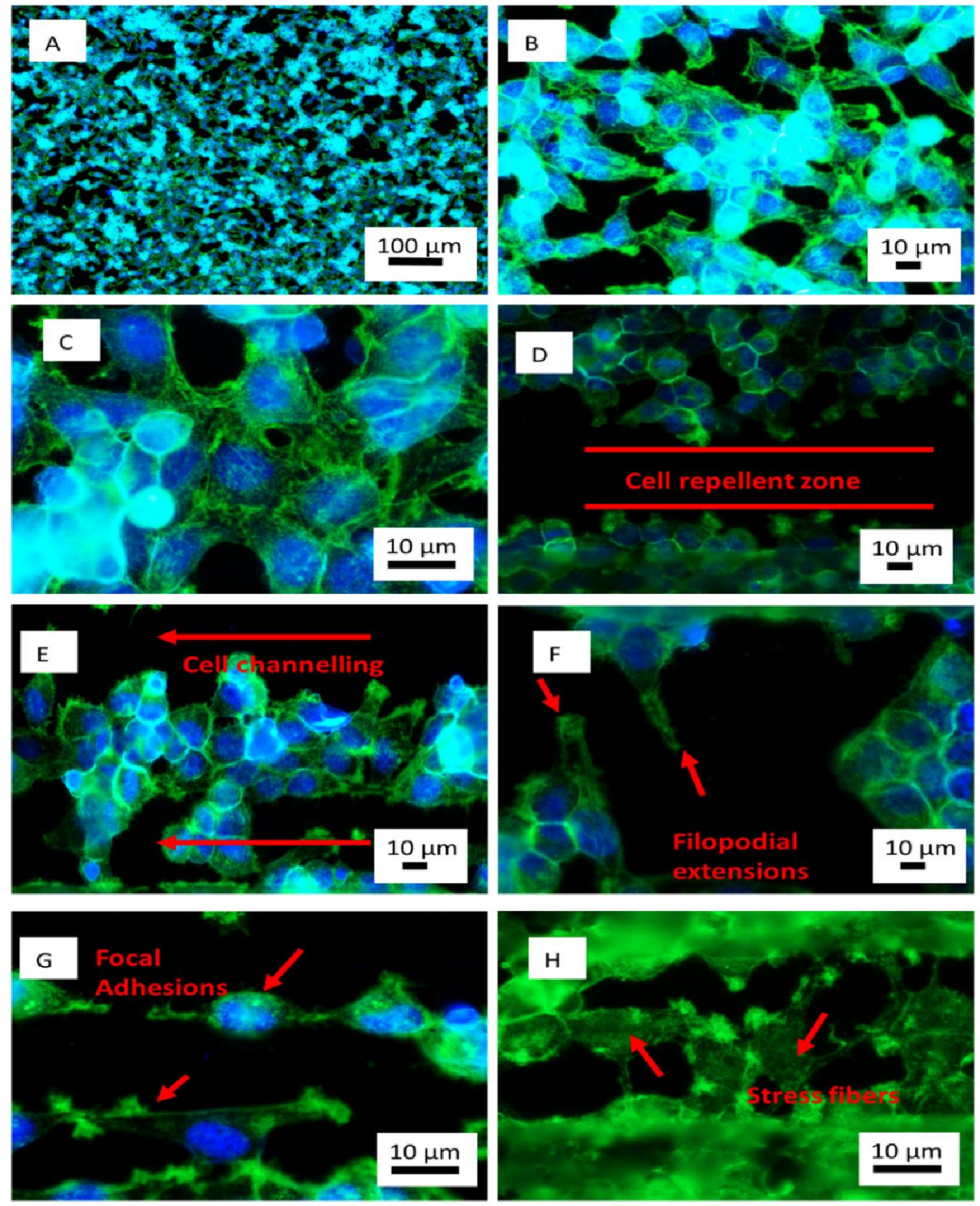

Figure 6-6: Fluorescence images of HeLa cells stained to reveal actin cytoskeleton and nucleus. (A-C) Cells growing on control silicon in a random fashion. (D) Low frequency and short pulse width after 48 hours of growth presenting cell repelling from functionalized zones(E) Cell channelling, cells growing in the same direction after 48 hours, low frequency and short pulse width (F) Filopodial extensions sensing away from the functionalized zone at high frequency and short pulse width after 24 hours of growth $(\mathrm{G})$ Focal adhesions forming at points away from functionalized zones at 48 hours, mid frequency and long pulse width $(\mathrm{H})$ Formation of stress fibers in cells growing near functionalized zones at 48 hours, mid frequency and long pulse width. 


\subsubsection{Cell Directionality (Cell polarization)}

Functionalized zone has shown that it is capable of generating cell migration in specific directions. This property is due to varying cues offered by the substrate in gradients. Owing to the adherent nature of the cells, it is apparent that contact guidance plays a vital role in the direction of migration of cells. Previous studies on directional cell migration have concentrated on substrates with topographical changes [176]. It is seen that grooves and patterned surfaces promoted directional growth. However, the functionalized surfaces present no changes in topography. The phase transformation of the functionalized zones take place at a sub-surface level. Therefore, the promotion of directional cell migration is attributed to presence of varying phases of silicon oxides and silicon. The formation of different phases of $\mathrm{SiO}_{2}$ and $\mathrm{Si}$ indicates varying surface energy on the functionalized zones. Different surface energies further induce varying levels of wettability. Consequently, passive interaction forces act on the cells to stimulate adhesion such as Van der waals forces. However, specific adhesion required for healthy growth of cells is not achieved in these areas. As a result, cells that grow away from this region are flatter with their cytoskeleton adhering well to the substrate. While cells react to external cues to such as surface energy, internal cues also dictate the polarization of the cell. It has been postulated that cells adopt a polarized nature to sustain free minimum energy in a metastable state[177]. It is for this reason that cells grow in a polarized fashion adjacent to the functionalized zone. Dickinson et al. stated that while quantity of adhesion ligands dictates the speed of cell locomotion, its spatial arrangement results in directional cell growth [178]. Finally, Raeber et al. investigated a similar trend with adherent fibroblasts and concluded that cells migrate parallel to the principle strain axis, consistent with our results[179].

\subsubsection{Cancer cell repelling due to haptotaxis}

Haptotaxis is the migration of cells in response to thermodynamic nature of the substrate [173]. The interaction of ultrafast pulses in the nanosecond regime with silicon results in areas of functionalization with varying thermodynamic states. This change in material properties includes the introduction of stress to this substrate. This stress is then sensed by the cells itself which results in the reorganization of the stress fibers, changes in focal adhesions and hence haptotaxis of the cells away from the functionalized zones. Further, a gradient of surface energies is a contributing factor. Schakenraad et al. stated that higher surface energies lead to higher adhesion 
of HeLa cells[180]. Therefore, is it is suggested that at a 48 hour period, the cells move to areas of higher surface energy, i.e. away from the functionalized zones. For this reason, even though cells initially attach close to functionalized zones at 24 hours, the lamellipodium is seen moving away. Another reason for initial attachment of the cells to the functionalized zone may be attributed to the attraction between a positive surface and primary amine groups which are later

overridden by other contributing factors. A reason why the lamellipodium is seen moving away from the functionalized zones may be due to the activation of repulsion receptors [181]. It is suggested that for cells to be repelled, the actin and microtubule dynamics are changed while simultaneously reducing adhesion between cells and the substrate itself.

\subsection{Summary}

Silicon is employed substantially in cancer diagnostics, yet its potential use as a cancer manipulative surface has not been explored. Silicon is innately a biocompatible surface and research has been focussed on its proliferative nature. The synthesis of programmable functionalized silicon generated through ultrafast laser pulse interaction is introduced. The generation of rare phases of silicon and silicon oxides and its stability at ambient temperatures is observed. This results in altered surface chemistry along a gradient which stimulates change in adhesion and migration characteristics of cancer. It is observed that the cancer aggressively seeks out areas away from the functionalized zones. This is evidenced in the organization of stress fibers, nucleus shape as well as the actin cytoskeleton. Further, high resolution SEM images demarcate filopodial attachment and sensing away from the functionalized zones. The results from this research study present significant results with precise manipulation of selective cell populations. Till date, the functionalized properties of silicon have not been examined. A versatile technique that does not involve any additive processes or change to the topography of silicon is presented. These unique results pave the way for a new path in cancer research. Further development of functionalized silicon will provide the impetus required to change how cancer research is currently being perceived. 


\section{Chapter 7}

\section{Engineering next generation functionalized multi-phase silicon/silicon oxide nano biomaterials to passivate the aggressive proliferation of cancer}

This chapter introduces engineered next generation in silicon nano biomaterials that are a combination of several phases of silicon and its oxides showing cancer manipulating properties. Currently, the use of nano silicon in cancer therapy is limited as drug delivery vehicles and markers in imaging, not as manipulative/controlling agents. This is due to limited properties that native states of nano silicon and silicon oxides offers. The engineering of next generation, nanofunctionalized multi-phased silicon/silicon oxide biomaterials is presented. This engineered biomaterial is synthesized via ultrashort pulsed laser synthesis with tunable properties that possess inherent drug-like cancer controlling properties that can passivate the progression of cancer. This nanostructured biomaterial is composed of individual functionalized nanoparticles made of a homogenous hybrid of multiple phases of silicon and silicon oxide in increasing concentration outwards from the core. The chemical properties of the proposed nanostructure such as number of phases, composition of phases and crystal orientation of each functionalized nanoparticle in the three dimensional nanostructure is defined based on precisely tuned ultrashort pulsed laser-material interaction mechanisms. In addition, physical characteristics such as functionalized nanoparticle size and density of three dimensional nanostructure is controlled by ultrashort pulsed laser-material interaction mechanisms. The hybrid nature of the functionalized nanoparticles in the three dimensional nanostructure is owing to the mixture of varying amorphous and crystalline phases of both silicon and silicon oxides forming a fused homogenous core-shell like structure with a tunable crystalline core and amorphous phase shell. The combination of varying phases of silicon and silicon oxides passivates the growth of cancer owing to the deactivation of the proliferative nature of silicon. The amorphous rich phased biomaterial show a 30 fold (95\%) reduction in cancer cell proliferation in 48 hours. Further, the size of the cancer cells reduces by $76 \%$ from 24 to 48 hours. An in-depth study is conducted on health of cancer cells on the nano-functionalized, multi-phased silicon/silicon oxide biomaterial 
and it is observed that nucleus as well as lamellipodia shrink on high density three dimensional nanostructured biomaterial. The control of cancer cells is a direct result of surface chemistry of each individual functionalized nanoparticle and the three dimensional topography of the biomaterial. This chapterpresents a unique and singular method to fabricate a homogenous multiphased silicon/silicon oxide biomaterials that possess unique cancer controlling properties. This method exposes untapped properties of combination of multiple phases of silicon and silicon oxides by precisely tuning the magnitude of phase transformation. This new insight on applications of multiple phases of silicon/silicon oxides in passivating cancer cells is a paradigm shift in current cancer research trends.

\subsection{Introduction}

The advent of nanotechnology has introduced nanoparticles and other nanostructures to the forefront of cancer therapy. However, the use of nanoparticles is limited as drug delivery carriers and markers in targeted therapy [182], [183]; and nanostructures are exclusively researched as biosensors for cancer detection [184], [185]. Further, biocompatible elements like nano silicon have only been researched as drug delivery agents, potential markers for imaging or diagnostic chips[186]-[188]. Therefore, it is observed that the applications for silicon nanoparticles are limited due to usage in its pure form and absence of modulated phase and composition of phases of pure silicon and silicon oxides.

Extensive studies revealed the limited scope of silicon and silicon oxides in cancer therapies. Silicon based nanoparticles have been applied to the field of imaging. Erogogbo et al. generated biocompatible luminescent silicon quantum dots by laser pyrolysis followed by etching until a required size is obtained [187]. These quantum dots were used as labels for pancreatic cancer cells. In addition to imaging, detection is another area of cancer therapy where silicon nanoparticles have been employed. For example, silicon nanowires owing to its electrical and biocompatible properties were used for biomarker detection[189]. Silicon nanoparticles are also employed as photosensitizers against cancer cells via photodynamic therapy. For instance, Xiao et al. formulated silicon nanoparticles via electrochemical etching from a single silicon crystal [161]. They were able to show $45 \%$ cancer cell death compared to $25 \%$ cell death where no nanoparticles were employed. It is observed that in all the studies, silicon has only been 
employed as a nanoparticle. Further, the use of an external agent such as radiation is necessitated.

Research in the area of drug delivery has also employed silicon/silicon oxide nanoparticles, focusing on porous silica nanoparticles as potential drug carriers. This is owing to several advantages that mesoporous silica offers like high stability, being chemically versatile and biocompatibility[188]. Further, it is a good specimen for controlled release of drugs [190].For instance Meng et al. developed mesoporous silica nanoparticles that can be loaded with Doxorubicin and P-Glycoprotein siRNA that would aid in cancer cell killing[191]. Sol-gel was employed to synthesize the mesoporous silica nanoparticles in Meng et al's study. These nanoparticles have dual characteristics of eluting chemotherapeutic drugs as well as siRNA capable of reducing drug resistance. Similarly, Rosenholm et al. synthesized a hybrid mesoporous silica nanoparticle with poly(ethylene imine) and different target moieties[192]. Solgel technique was employed in their study in addition to surface polymerization to synthesize these nanoparticles for targeted therapy. Zhang et al. synthesized mesoporous silica nanoparticles that target cancer cells selectively due to the addition of folate targeting agents[193]. Similar to silicon nanoparticles used in imaging, drug delivery also needs additional agents such as drugs like Doxorubicin to control the growth of cancer. Further, there have not been studies to demonstrate the applicability of nanostructured biomaterials in controlling the growth of cancer.

In addition, to requiring an external agent and absence of nanostructured biomaterials, silicon is employed in its pure form and has limited scope. To the best of our knowledge, there have been no studies that explore the combination of a homogenous phases of silicon and silicon oxides as potential cancer controlling agents in a three dimensional nanostructure. The generation of these phases deactivates the inherent proliferative nature of silicon.

In this chapter, next generation in silicon nano biomaterials is introduced. The generation of multi-phased silicon/silicon oxide nano biomaterials that are a fibrous aggregation of functionalized nanoparticles on the surface of silicon via ultrashort pulsed laser synthesis is reported. The functionalized nanoparticles in the multi-phased nano biomaterial form a core-shell like structure with a combination of homogenous phases of silicon and silicon oxides. The unique aspect of ultrashort pulsed laser synthesis method lies in the fact that each single functionalized nanoparticle can be modified in its chemical and physical form. When the 
ultrashort pulsed laser with pulse to pulse separation time between micro and nanoseconds interacts with the surface of silicon, it vaporizes silicon. Subsequently, these species nucleate, coalesce and aggregate to form three dimensional nanoparticle aggregates. It is observed that each of these functionalized nanoparticles in the three dimensional nano biomaterial are made up of a crystalline silicon core and is enveloped by an increasing concentration of silicon oxides. The phase as well as size of the functionalized nanoparticle is controlled by altering the plume dynamics, controlling the surface and plume temperature during ultrashort pulsed laser-material interaction. It is observed that this novel multi-phased nano biomaterial deactivates the proliferative nature of silicon and passivates cancer cell growth. The growth of cervical cancer cells on this functionalized multi-phased nano biomaterial exhibit remarkable properties of cell proliferation control. A 95\% (30 fold) decrease in cancer cells is observed on amorphous rich nanofibers produced at the shortest pulse duration. The health of the cell is also quantified presenting drastic reduction in cancer cell size at the end of 48 hours on nanostructured silicon with decrease in nucleus as well as lamellipodia size. This reduction is attributed to the internalization of nanoparticles and encapsulation of cells. Further, previous studies of similar nano materials has exclusively characterized its proliferative properties [3], [80], [194]. On the contrary, this study presents selective cell death based on the homogenous fusion multiple phases of silicon and silicon oxide. Therefore, this next generation nano biomaterial has the capability to expose drug-like properties of this silicon/silicon oxide that has never been shown before. This has vast applications in implant technologies, drug delivery systems and microchip devices.

\subsection{Experimental Detail}

\subsubsection{Generation of multi-phase nanostructure}

The samples are irradiated using a diode pumped, Yb-doped femtosecond laser system (ClarkMXR Inc. IM-PULSE Series Ultrashort Pulse laser) at 4,8and $26 \mathrm{MHz}$. The laser irradiates the samples in an array of lines at distances between $100 \mu \mathrm{m}$ to $2 \mathrm{~mm}$. The speed at which the laser irradiates and the power of the laser is maintained at $10 \mathrm{~mm} / \mathrm{s}$ and $15 \mathrm{~W}$ respectively. The laser pulse width or peak power plays an important role and is varied between $214 \mathrm{fs}$ (short pulse), $714 \mathrm{fs}$ (medium pulse) and $1428 \mathrm{fs}$ (long pulse) corresponding to $8.76 \mathrm{MW}, 2 \mathrm{MW}$ and $1.31 \mathrm{MW}$ peak power respectively. All the parameters are controlled by a computer to facilitate precision and accuracy. 


\subsubsection{Morphological characterization of multi-phase nanostructure}

Scanning electron microscopy (SEM) (Hitachi S 5200) is performed to characterize the morphology of the multi-functionalizednanostructures. The samples are loaded onto aluminum stands and gold coated for SEM. Following SEM, Energy-dispersive X-ray spectroscopy (EDX) is carried out to determine the elemental composition of irradiated silicon. Micro-raman spectroscopy as well as X-Ray photon spectroscopy (Thermo scientific K-alpha) is used further to study surface composition quantitatively and qualitatively. X-ray diffraction(Siemens D5000 conventional theta/2theta diffractometer) is used as a secondary tool to confirm elemental composition.

\subsubsection{Cancer cell- multi-phase nanostructure interaction}

Cervical cancer cells (HeLa, ATCC, USA) are employed to qualitatively and quantitatively study cancer controllability. The cells are grown in DMEM/F12 medium supplemented with 10\%fetal bovine serum and 1\% Pen-strep. The nanostructured siliconis kept under UV light for 20 minutes. Subsequently, the substrates with nanostructures are placed in petri dishes with HeLa cells at a density of $10^{5}$ cells $/ \mathrm{ml}$, totaling $3 \mathrm{ml}$ per dish. The petri dishes are placed in an incubator for 24 and 48 hours.

\subsubsection{Statistics}

All experiments are carried out in triplicates and the data points are averages unless otherwise mentioned. The error bars indicate standard deviations.

\subsection{Results and Discussion}

\subsubsection{Synthesis of multi-phase nanostructure}

The interaction of ultrashort laser pulses results in the formation of novel nanostructures that are an aggregation of nanoparticles forming nanofibers, comprising of silicon as well as silicon oxides (Figure 7-1). The formation and composition of these nanostructures is determined by varying plume dynamics. When a single laser pulse strikes the surface of silicon, the region is heated followed by heat dissipation through the lattice. The number of atoms that evaporate from the surface after single pulse interaction is low [195]. However, in this research study, similar to current industrial trends, multiple pulses are employed. The frequency of the multiple pulses is 
precisely controlled between 4 and $26 \mathrm{MHz}$. This frequency determines the separation time between the pulses which varies from micro to nanoseconds. When the pulse to pulse separation is reduced, there is not enough time for the heat to dissipate and is concentrated at the zone of laser-material interaction. Further, another parameter to consider is the speed of the laser which is maintained at $10 \mathrm{~mm} / \mathrm{s}$. Therefore, a single spot receives multiple pulses for $1 \mathrm{~ms}$. Tavangar et al. showed that the density of ablated material is dependent on the pulse to pulse separation time (frequency of pulses) as well as the laser-material interaction time using the following equation [196]

$N_{M P} \approx n_{\text {air }}\left(\frac{k_{B} a A t_{e q}^{\frac{3}{2}}}{M_{a} k_{h} \pi^{\frac{3}{2}}}\right)^{\frac{1}{2}}\left(P_{\text {avg }} R_{r e p} A_{f o c}\right)^{\frac{1}{2}} D_{t}[$ atoms $]$

where $R_{\text {rep }}$ is the frequency of pulses, $\mathrm{P}_{\mathrm{avg}}$ is average power, $\mathrm{R}_{\text {rep }}$ is frequency of pulses, a is thermal coefficient of silicon, $A$ is laser absorption coefficient of silicon, $\mathrm{n}_{\text {air }}$ is number density of electrons in air, $A_{\text {foc }}$ is laser spot size, $\mathrm{t}_{\mathrm{eq}}$ is equilibrium time, $\mathrm{k}_{\mathrm{B}}$ is Boltzman constant, $\mathrm{k}_{\mathrm{h}}$ is the heat conduction coefficient and $D_{t}$ is the laser material interaction time. It is evident from the above equation that the pulse to pulse separation time plays a critical role in the density of atoms that is ablated from the surface. The ablated density is directly proportional to the density of nanostructuresthat are synthesized on the surface. In this study, it is evident that the density of nanostructures plays an important role in controlling the growth of cells.

The synthesis of these nanostructures can be explained by understanding mechanisms in the plume. Specifically, based on the size of nanoparticles it is apparent that vapor condensation mechanism is responsible for formation. The cooling of the plume is initiated by diffusion in ambient air. The ablated matter is considered to be in a supercritical fluid state owing to above threshold fluence of the laser [197].At the end of a laser pulse, ions interact to form molecules which collide due to thermal motion. When the temperature falls, the formation of nanoparticles begins[198]. The formation of nanoparticles is followed by coalescence and coagulation. And finally aggregation of coagulates takes place. 


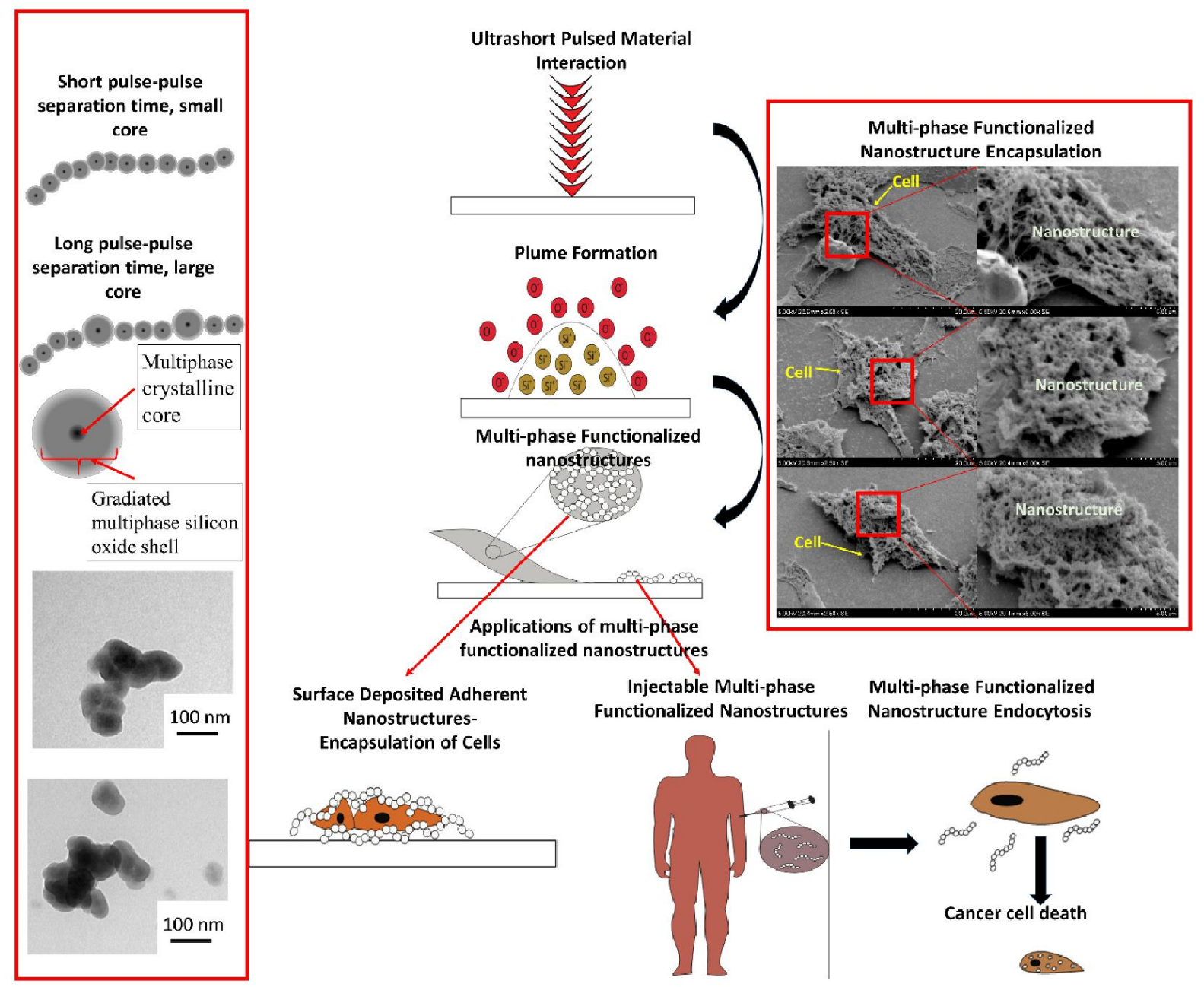

Figure 7-1: Illustration of mechanism of formation of multi-phase silicon/silicon oxide nanostructures potential applications.

This aggregation may exist between previously aggregated species or an aggregated species and atoms. While the process of formation of nanoparticles in the nanostructures remains the same, the size of the nanoparticles varies according to frequency of incoming pulses (Figure 7-2(A-D)). It is evident, that the nanoparticle size on average decreased with an increase in number of pulses. Further, Sivayoganathan et al. showed a unique bimodal distribution of silicon nanoparticles at lower frequencies [116]. Even though the average size of the nanoparticles stayed constant, lower frequencies had a small fraction of nanoparticles that were larger. This could be attributed to the presence of neutral species in addition to ionized species in the plume. 
The SEM images also provide an insight into the density of the nanostructures (Figure 7-2(E-H). It is observed that zones away from the area of laser material interaction have proportionally lower density of nanostructures. In addition to the density of nanostructures, the morphology of nanostructures is also significantly different as we move further away, the nanostructure changes to fragments of agglomerated nanoparticles.

\subsubsection{Composition of multi-phase nanostructure}

Subsequent to the study of morphology of the nanoparticles in the nanostructure, the composition is studied. The study is conducted based on EDX, XRD and Raman analysis. EDX shows the presence of oxygen in areas deposited with nanostructures (Figure 7-3(A, A1, A2).EDX is used only as a preliminary qualitative tool of analysis due to sharp peaks of substrate silicon distorting data. Raman analysis was performed to qualitatively assess the different phases present (Figure 7-3(B)). The image shows the presence of various phases of silicon in the nanostructures. The Raman micrograph reveals the broadening of peaks at $26 \mathrm{MHz}$ which may is potentially due to the introduction of amorphous silicon. Further, the peak signifying silicon has been shifted from $520 \mathrm{~cm}^{-1}$ on bulk to $516^{-1} \mathrm{~cm}$ at all parameters. This is an indication of the particle sizes which can be calculated by[199]

$d=2 \pi\left(\frac{B}{\Delta \omega}\right)^{n}$

Where $\mathrm{d}$ is the crystal diameter and is calculated to be approximately $4.43 \mathrm{~nm}$ when $\mathrm{B}=2 \mathrm{~cm}^{-}$ ${ }^{1} \mathrm{~nm}^{2}$ and $\Delta \omega$ is the shift in peak. 

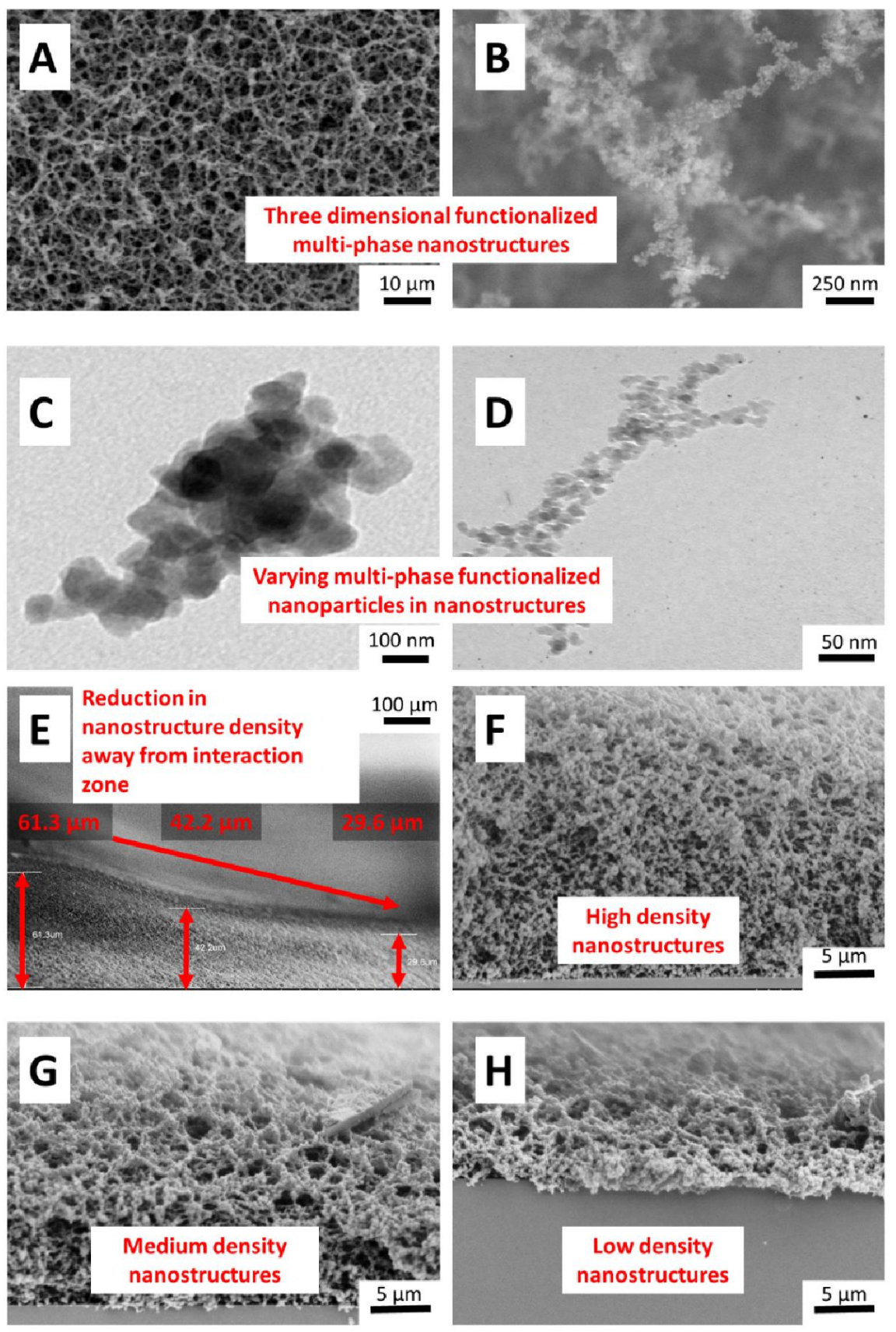

Figure 7-2: (A-B) Magnified and zoomed SEM images of multi-functionalized nanostructured silicon (C-D) TEM images showing various nanoparticle sizes (E-H) SEM micrographs of varying nanostructure density of multi-functionalized nanostructured silicon.

Further analyses on the peaks reveal irregularities in the width of the peaks. This is attributed to defects in grains being produced in the nanoparticles. This is also a potential indication on polycrystalline silicon. Another interesting fact is also observed in the Raman micrographs. The 
peaks are broader towards higher Raman shifts for short pulse and broader towards lower Raman shifts for long pulse fs. This disparity is due to the difference in defects being introduced at different pulse durations.

To further study the composition of this nanostructure XRD is employed (Figure 7-3(C)). XRD reveals the presence of three distinct crystal structures of silicon, Si (111), (220) and (311).These crystal structures are observed at all parameters. However, the presence of amorphous oxides depends on the frequency of pulses. It is seen that as the pulse to pulse separation time is reduced or the frequency of pulses is increased there is a corresponding increase in amorphous oxides. It is seen that the nanostructures contain randomly oriented micro crystallites of silicon. This nature of randomly oriented micro crystalline structures could be attributed to varying plume mechanisms. The collisions of species with different crystal orientations may result in such attributes [197].

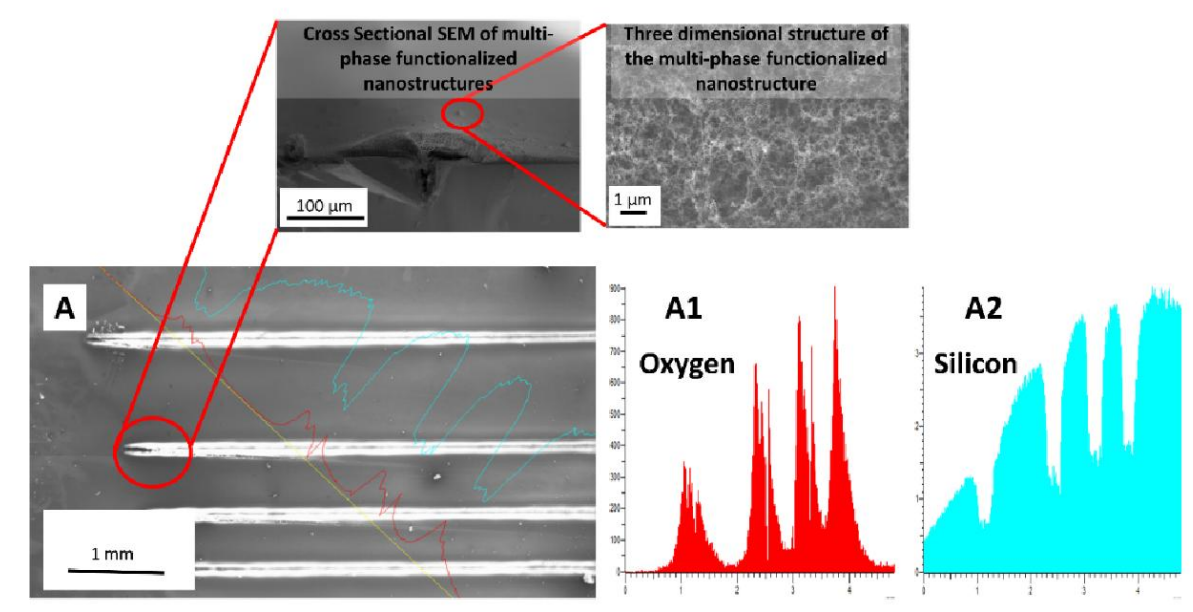

Figure 7-3: (A) SEM micrograph depicting ultrashort pulsed laser-material interaction zones. The magnified images show the nanostructure density at point of interaction and away (A1) Oxygen content (A2) Silicon content 


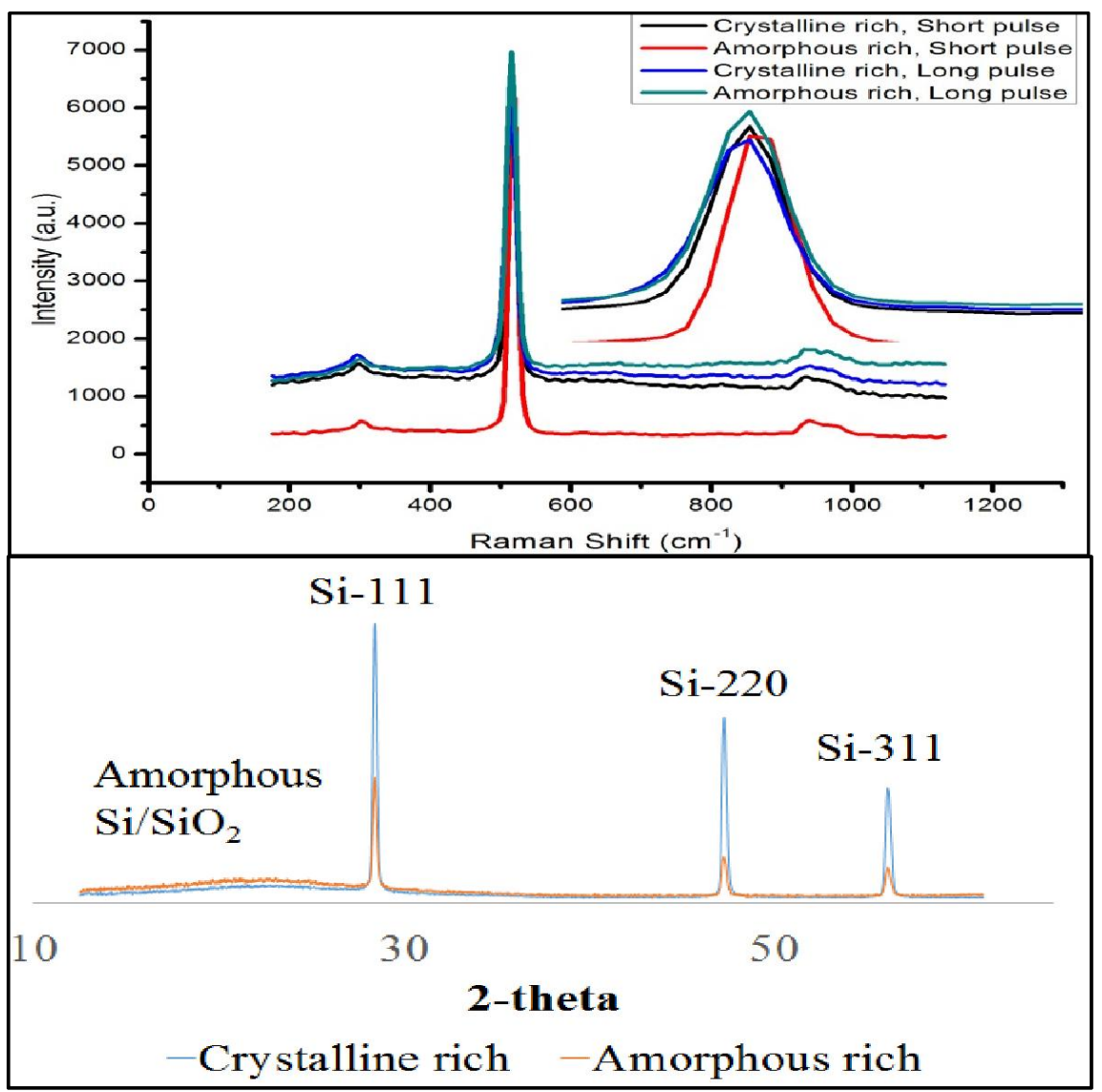

Figure 7-4: Raman spectroscopy graphs at varying pulse durations and different phase concentrations and XRD graphs at different phase concentrations.

The mode of formation of the nanostructures is described based on different plume mechanisms.At long pulse to pulse separations, there is high crystal concentration compared to amorphous oxides. This is due to slow cooling of liquid crystal droplets.At shorter pulse to pulse separation times, there is not enough time for the species to cool and hence the introduction of amorphous oxides takes place. It is hypothesized that the amorphous oxides are formed as an external 'shell' surrounding the crystalline silicon.At shorter pulse to pulse separation times, more species are ejected into the plume. Further, ionization of gases around the plume also takes place. Therefore, there is a higher chance of silicon and oxygen collisions further promoting the formation of amorphous silicon oxide shells[200]. The size of the nanoparticles also depends upon similar tendencies of the nucleation of species. Higher density promotes more nucleation 
sites encouraging smaller nanoparticles. A lower density of species causes fewer nucleation sites with bigger nanoparticles.

\subsubsection{Cancer cell manipulation}

\subsubsection{Adherent multi-phase nanostructure}

Surface-bound nanostructures adhere strongly to the surface of silicon owing to high temperature formation in the plume. These nanostructures form a three dimensional matrix which is interlinked and randomly oriented. In contradiction to other research studies that present cell proliferation of nanofibrous structures, this study shows reduction in the rate of cell proliferation[201]. Silicon substrateswith their nanostructures ultrasonically removed are considered as control samples. As evident in Figure 7-4, there is approximately a 95\% reduction in the growth of cells on amorphous rich nanostructures fabricated at short pulse. Moreover, there is a reduction in the number of cells on any surface that has surface-bound nanostructures. The disparity in reduction of cells could be attributed to encapsulation of cells in the amorphous rich nanostructures fabricated at short pulse. The cell proliferation and health is further analyzed based on the density and phase of fibers.

\subsection{Cell proliferation: Role of density of nanostructures}

The synthesis of nanostructures causes differences in its phases and density. As mentioned previously, the density of nanostructures closest to the zone of laser material interaction is significantly higher and will consist of high temperature and high pressure phases. To analyze the effects of density on the growth characteristics of HeLa cells, cell count as well as cell health are assessed at both 24 and 48 . It is observed that at 24 hours, more number of cells have attached to the high density fibers compared to low density fibers. There is no clear indication of differences in cell attachment at different pulse durations. However, while comparing cell attachment at 24 hours, it is evident that the number of cells that attached at $4 \mathrm{MHz}$ (Crystalline rich) are significantly higher than those that attached at 26MHz (Amorphous rich)(Figure 7-4(AB). This unique tendency of cell growth is attributed to the morphology and composition of the nanostructures. 

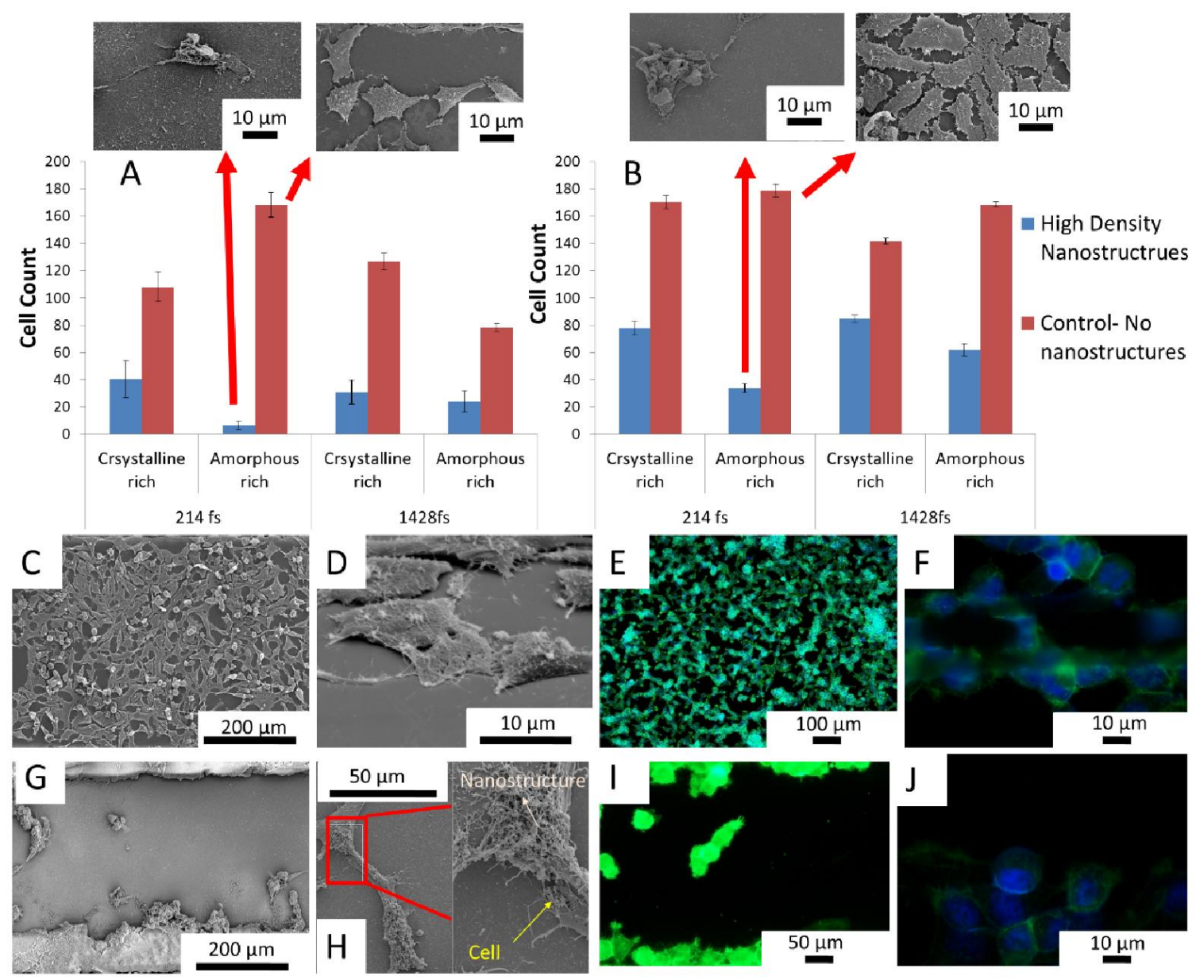

Figure 7-5: Number of cells on different phases of nanostructures as well as different pulse generated nanostructures. Comparison is made between cells on high density nanostructures and control or virgin silicon at (A) 24 hours and (B) 48 hours. (C-D) SEM images of HeLa cells on control or virgin silicon (E-F) Fluorescence microscopy images of HeLa cells on control or virgin silicon (G-H) SEM images of HeLa cells on high density nanostructures (I-J) Fluorescence microscopy images of HeLa cells on high density nanostructures. All images are of cell growth at 48 hours.

After 48 hours of cell growth, there is evidence of change in cell attachment characteristics. There is a complete reversal of cell attachment with respect to density.There are significant increases in cell growth on low density fibers compared to high density fibers. This is comparable at all parameters. However, it is still apparent that amorphous rich phases presents lower cell attachment compared to crystalline rich phases.But an interesting phenomenon occurs 
here where percentage cell proliferation is completely a reversed between amorphous rich and crystalline rich phases. At 48 hours, it is clear that the rate of proliferation is consistently higher for amorphous rich phases compared to crystalline rich phases.

Therefore, it is seen that cells are affected by multiple parameters that are each controllable by varying laser parameters. An increase in density of nanostructures reflects the two aspects, an increase in nanostructures itself as well as an increase in high temperature phases. Nisbet et al. reviewed the response of neutrites on different densities of electrospun fibers[202]. They found a correlation between the distances of fibers at various densities dictated cell growth. Since the nanostructures are in fact composed of individual nanoparticles, it is postulatedthat this increasein the surface area improves protein adsorption and initial cell attachment. At 24 hours, cells preferentially attach to areas of higher protein adsorption, which in this case is the high density of nanostructures[203]. After initial attachment, it is hypothesized that the cells are trapped or encapsulated in the fibers and hence their growth potential is severely hampered. At the same time, the cells that are growing on low density of nanofibers are not encapsulated by fibers but rather the fibers forms points of adhesion. Therefore, there is a marked increase in cell proliferation in low density of fibers over a period of 48 hours. Further, it is been found that cancer cells are inherently predisposed to migrating through fibers to move to other areas and metastasize[204]. Due to this intrinsic feature, they are more likely to be encapsulated by high density fibers.

The phase of the fibers itself has a significant effect on the attachment characteristics of cells. From the XRD, it is clear that frequency of pulses or pulse to pulse separation time has a major influence on the crystalline vs. amorphous nature of nanostructures. Further, at $26 \mathrm{MHz}$, due to less pulse to pulse separation time more silicon species are formed in the plume. Consequently, an increase in the density of the nanostructures is observed. However, the nanostructures that are synthesized at $26 \mathrm{MHz}$ are not strongly bonded and due to this reason encapsulation of cells occurs. There have been previous studies that show that cells prefer a crystalline surface to attach on to compared to an amorphous surface [205]. 

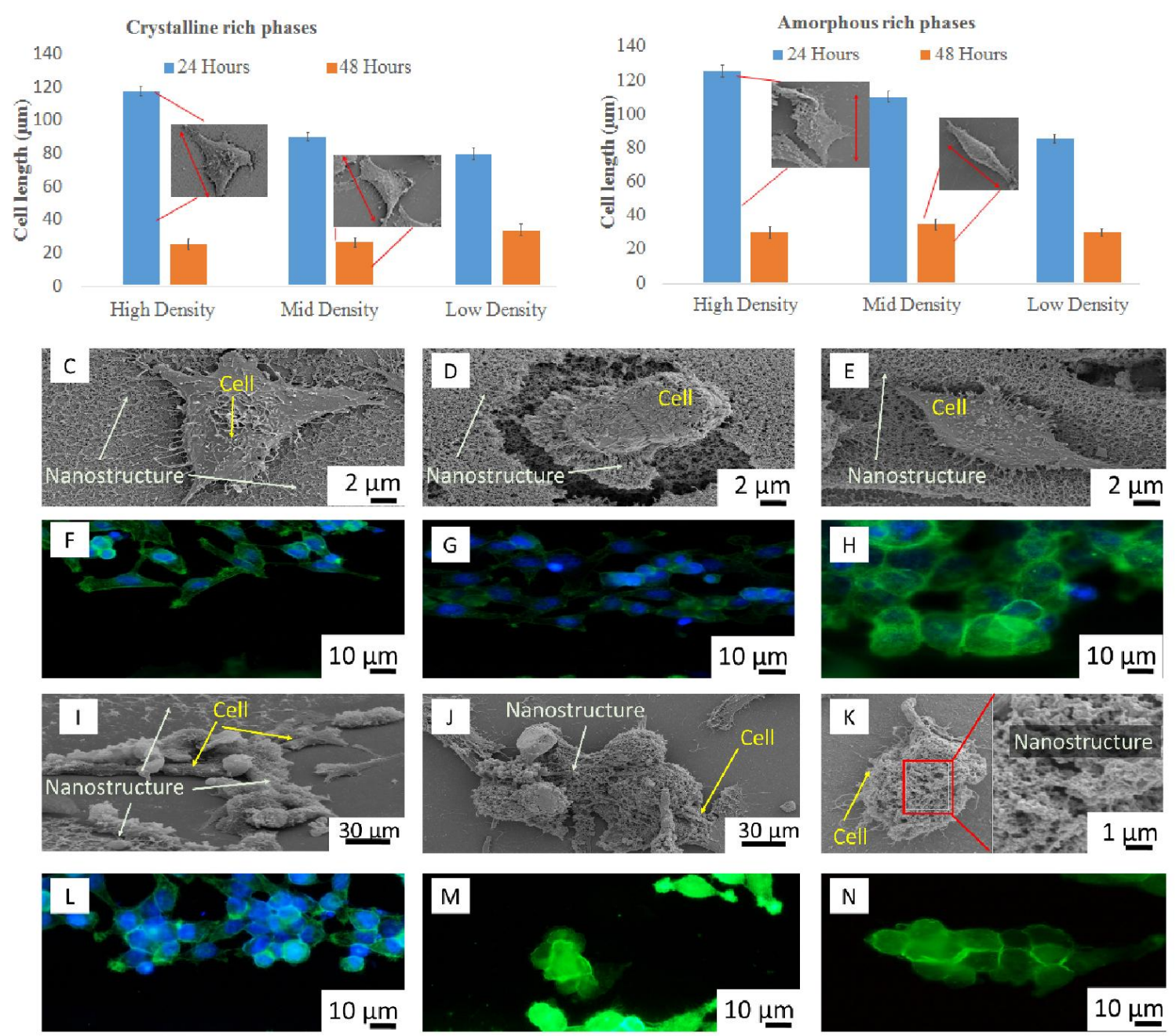

Figure 7-6: (A) The length along the major axis of HeLa at 24 and 48 hours on crystalline rich phases on varying densities of nanostructures (B) The length along the major axis of HeLa at 24 and 48 hours on amorphous rich phases on varying densities of nanostructures (C-E) SEM images of cell growth at 48 hours on crystalline rich phases $(\mathrm{F}-\mathrm{H})$ Fluorescence microscopy images of cell growth at 48 hours on crystalline rich phases (I-K) SEM images of cell growth at 48 hours on amorphous rich phases (L-N) Fluorescence microscopy images of cell growth at 48 hours on amorphous rich phases.

In addition to studying the number of cells present, the health of the cells of the cells is also considered(Figure 7-5). To quantify the health of the cells, the length along their major axis is calculated[95]. It is that evident that at 24 hours, irrespective of the density of nanostructures, the cells were longer on nanostructures that were amorphous rich. Cells also preferred high 
density nanostructures compared to low density nanostructures. A remarkable phenomena occurs after 48 hours. The length of the cells is sharply reduced to lesser than $50 \%$ of its original length for both amorphous and crystalline rich phases.

A difference in density of the nanostructures causes a trend the in the way the cells attach to them. An increase in density reflects larger surface area for the cells to attach to[206]. Further, Zhao et al. proved that nanostructures induce higher amounts of collagen secretion as well as protein adsorption[207]. Therefore, at 24 hours cells are flatter on denser nanostructures. Amorphous rich phases showed better cell adhesion owing to two factors- particle size as well as higher concentration of nanoparticles. The drastic reduction in size that is observed at 48 hours maybe explained by passive ingestion of nanoparticles in the 48 hour period(Figure 7-6). Similar results were seen in a study conducted by Jin et al. where the fibroblasts showed a reduction in size when the concentration on nanotitanium was increased in the solution[208]. The process of internalization is described based on lysosome activity in the cells.

To study the cellular mechanism and adhesion, fluorescence microscopy is performed. Figure $5(\mathrm{~F}-\mathrm{H})$ and (L-N)present virgin silicon and nanostructured silicon and their interaction with HeLa. On control virgin silicon, cells grow randomly with well defined, flattened cytoskeleton. Focal adhesion points are observed at random points in the cell. These focal adhesion points are the interaction points of cells and the substrate. Stress fibers are cues for the cells based on mechanical signals from the substrate[174]. The density and organization of the stress fibers are an indication of the stress being transmitted by the substrate. The stress fibers on control silicon are randomly arranged. Interaction of $\mathrm{HeLa}$ cells with multi-functionalizednanostructured silicon, both adherent and deposited presents cells that are rounded and drastically reduced in size. Stress fibers are completely absent in cells growing on nanostructured silicon. Since stress fibers are an integral part of cell adhesion, it can be concluded that cells are apoptotic. The edges of the cells appear brighter signifying adhesion points. 

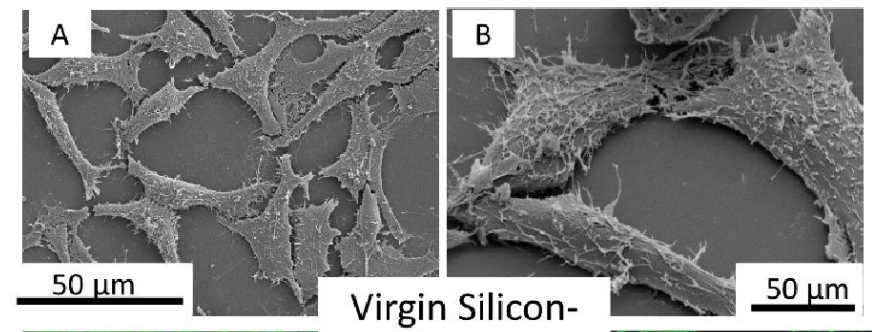

Endocytosis of multi-phase functionalized nanostructures leading to rounded cells
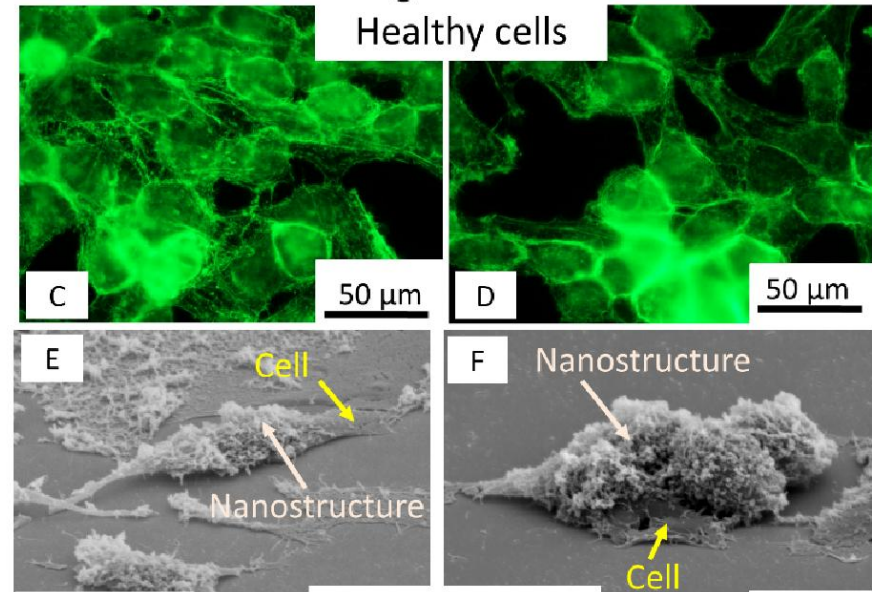

F Nanostructure

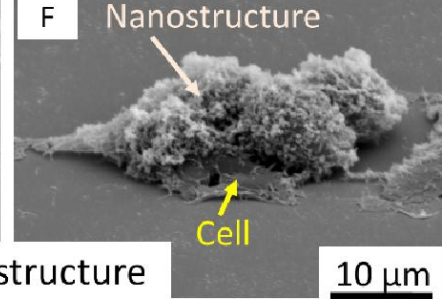

$50 \mu \mathrm{m}$ Nanostructure

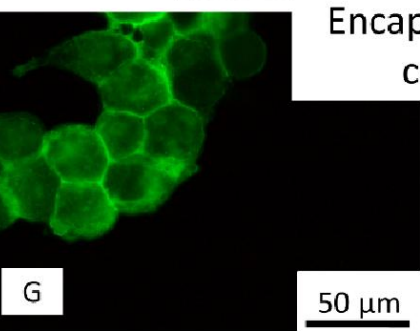
Encapsulated cells

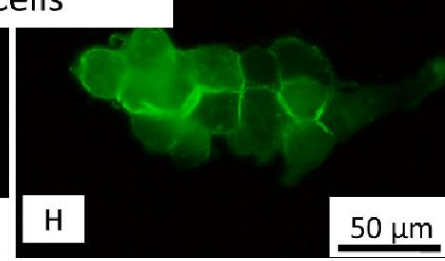

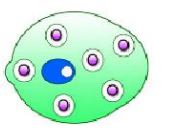

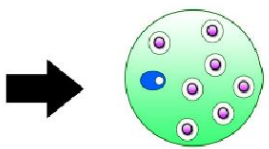

Complete rounding of cells owing to increasing concentration of nanoparticles in cell body

Figure 7-7: Mechanism of cell death. (A-B) SEM images of HeLa on control virgin silicon after 48 hours (C-D) Fluorescence microscopy images of HeLa on control virgin silicon after 48 hours (E-F) SEM images of HeLa on amorphous rich nanostructures after 48 hours (G-H) Fluorescence microscopy images of HeLa on amorphous rich nanostructures after 48 hours.

\subsection{Cell Health: Exploring the nucleus}

Comparing the nucleus between Figure 7-4(F) and $(\mathrm{J})$ it is evident that the nucleus is well rounded on cells growing on control virgin silicon as opposed to the nucleus of cells grown on nanostructured silicon. The elliptical or polarized shape of the nucleus is owing to actin cytoskeletal forces [175]. In addition, the size of the nucleus also significantly reduces with increasing multi-functionalizednanostructure density (Figure 7-8). It is hypothesized that a 
reduction in nucleus size may induce signalling that lead to apoptosis. Further, altered nucleus size may indicate changes in chromatin organization and gene expression [209].

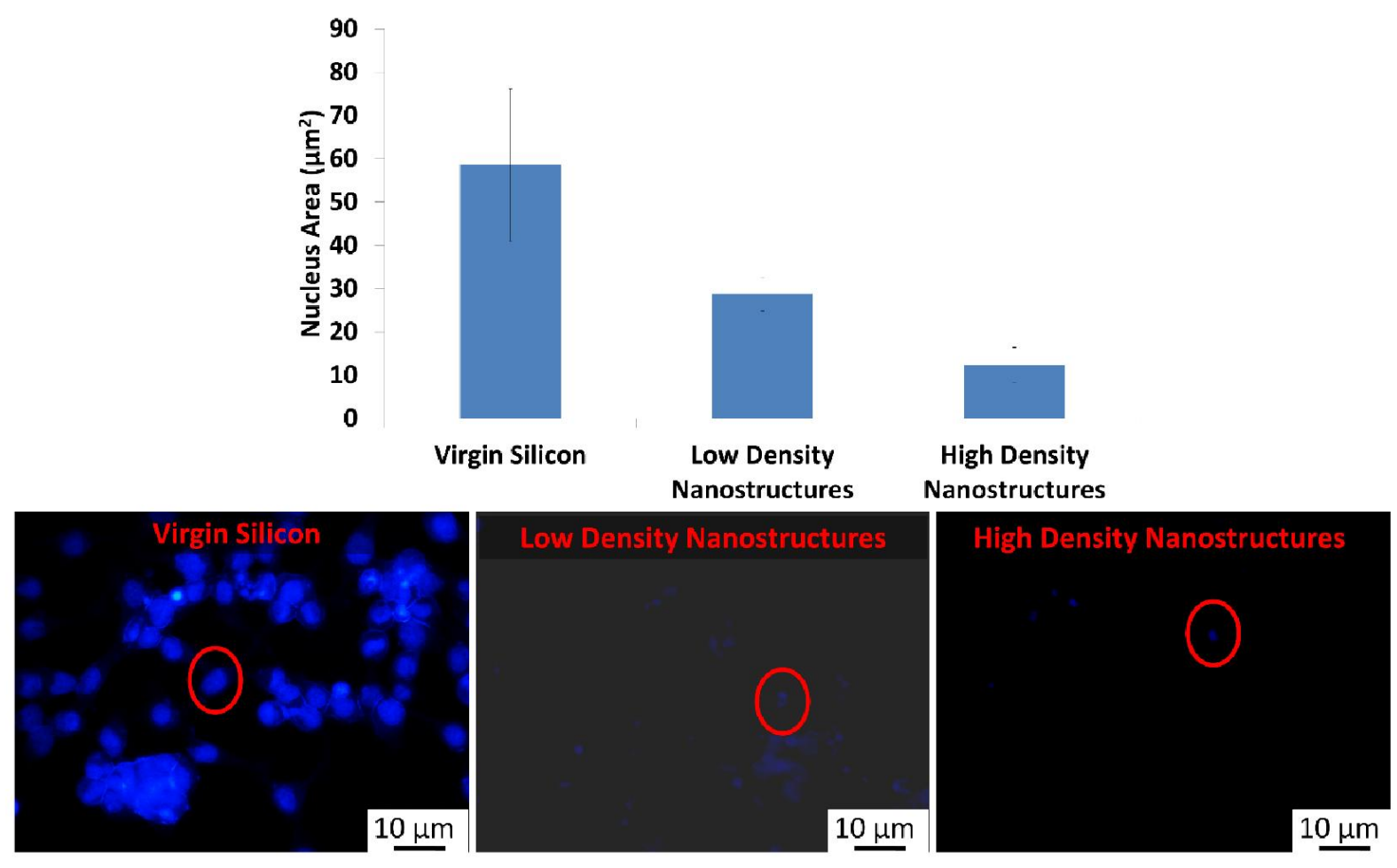

Figure 7-8: Measuring nucleus over low and high density nanostructures. The graph shows reduced nucleus size at high density nanostructures.

\subsection{Cell health: Characterizing lamellipodia}

The lamellipodia of cells on nanostructures and on control silicon is measured (Figure 7-8). It is observed that there is a significant difference in the formation and adhesion characteristics of lamellipodia on control and nanostructured silicon. Lamellipodia are sheet like extensions that protrude from the cell and aid in its migration. The presence of bigger lamellipodia points to higher adhesion and migration of the cells [210]. Figure 7-8 shows that larger areas of the lamellipodia attach to control silicon compared to nanostructured silicon. The changes in lamellipodial formation and attachment alter the growth characteristics of cells and alter its migration and proliferation routes [211]. 


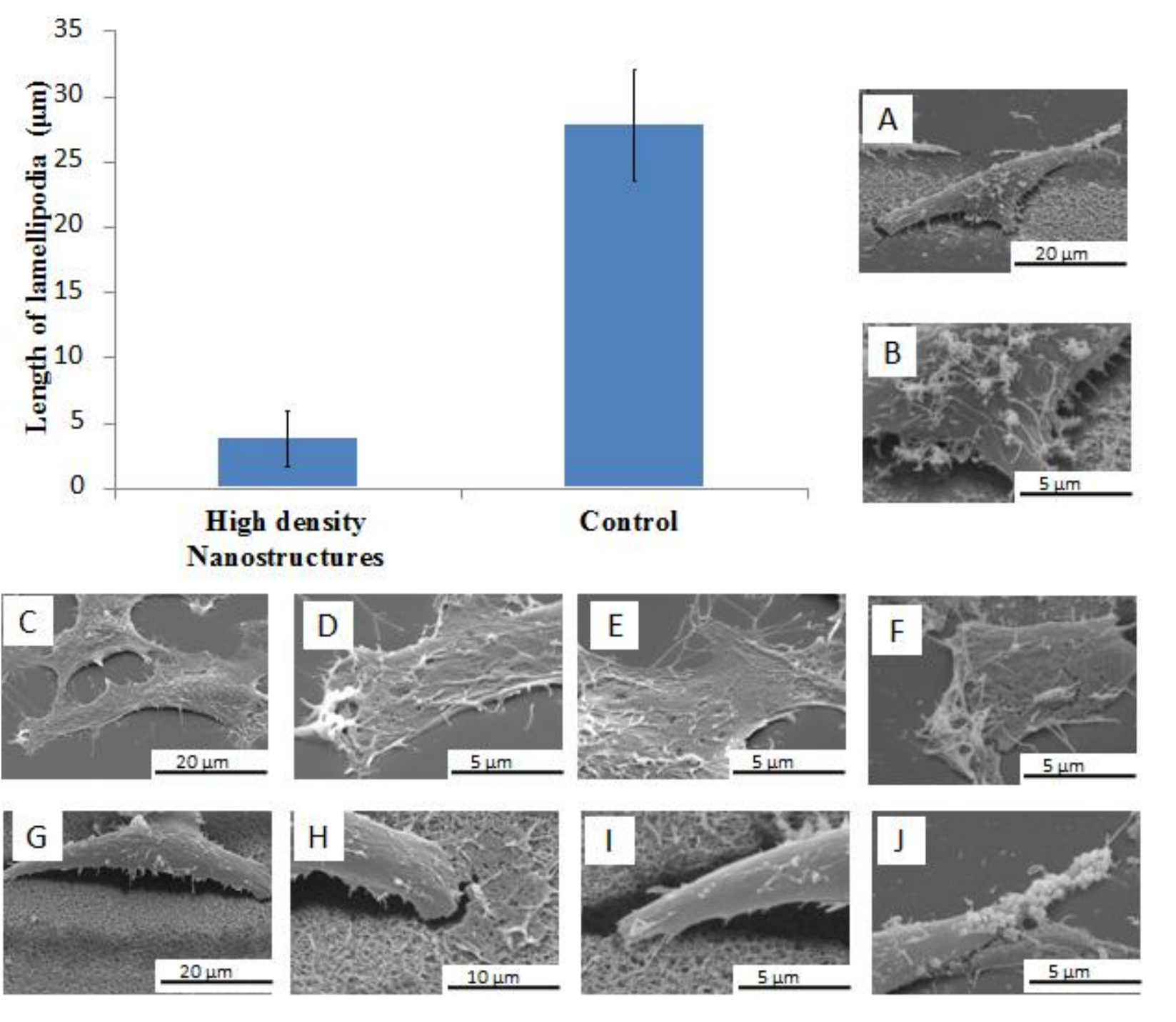

Figure 7-9: Graph depicts the lamellipodia length of control and high density nanostructures. (A, B, F, J) Illustrates a cell growing on nanostructures as well as control area illustrating lamellipodial adhesion on control silicon and less adhesion of nanostructures (C-F) Cell growth on control silicon (G-I) Cell growth in nanostructured silicon.

\subsubsection{Deposited multi-phase nanostructures}

The interaction of ultrashort laser pulses with silicon results in the formation of nanostructures at the interaction zone. Due to silicon's low threshold, the density of fibers formed is significant and is distributed through a wider area. The fibers that are formed away from the zone of interaction are formed at lower temperatures and hence do not strongly attach to the substrate. When these fibers are immersed in cell media they get dispersed from the site of formation. These are now considered 'floating' nanostructures. Due to their detached nature, they do not 
encapsulate cells but rather instigate apoptosis based on passive ingestion as showed in Figure 7-6. Both crystalline and amorphous nanostructures had an apparent effect on cells, however, like the adherent nanostructures, the deposited nanostructures elicited a further reduction in number of cells(Figure 7-9).
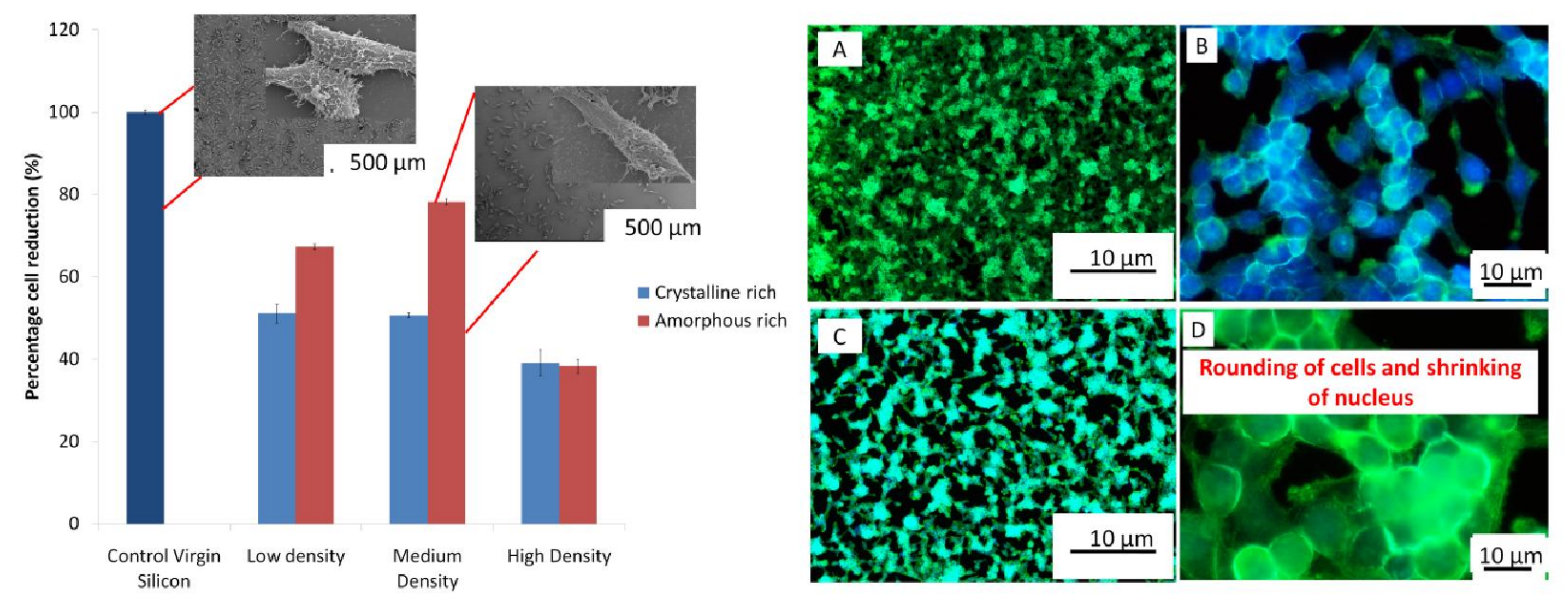

Figure 7-10: Percentage reduction of cells on deposited nanostructures. Significant reduction in number of cells is observed at high density of deposited nanostructures

\subsection{Summary}

This chapter reports the next generation in silicon nano biomaterials that are a homogenous combination of multiple phases of silicon and silicon oxides. Due to its limited scope, the use of pure silicon/silicon oxide based nano biomaterials in cancer therapy has been limited to drug delivery carriers, cancer marker detectors and as photosensitizers in photodynamic therapy. Through next generation nano-functionalized multi-phased engineered silicon/silicon oxide based nano biomaterials, novel drug-like properties that are capable of controlling the growth of cancer are presented. Ultrashort pulsed laser synthesis is employed to fabricate nanofunctionalized multi-phased nano biomaterial directly on the surface of silicon. The three dimensional nano biomaterial is composed of individual functionalized nanoparticles with a crystalline silicon core enveloped by increased concentration of silicon/silicon oxide shell. The unique aspect of this mode of synthesis is precise controllability of the number of phases, concentration of phases and size of the crystalline core enveloped amorphous surrounding. This is owing to precise control over the surface temperature and plume temperature that alters the ultrashort pulsed laser-material interaction mechanism. This novel functionalized multi-phased 
nano biomaterial deactivates the proliferative nature of silicon and passivates cancer cell growth owing to the unique blend of multiple phases of silicon and silicon oxide. The culture of HeLa cells on the functionalized multi-phased nano biomaterial shows a critical dependence of cancer cell proliferation on topography as well as material chemistry of functionalized multi-phased nano biomaterial. It is observed that there is a 30 fold $(95 \%)$ reduction in the number of HeLa cells after 48 hours on amorphous rich phases of multi-phased nanostructures. The nucleus as well as lamellipodia reduced in size significantly on high density of functionalized multi-phased nano biomaterial. Two hypothesis for reduction in number of cells is put forth- endocytosis of functionalized multi-phased nano biomaterial and encapsulation of cells by functionalized multiphased nano biomaterial. Therefore, this nano-functionalized multi-phased nano biomaterial diminishes cell proliferation unlike other research studies on pure silicon nanostructures that show the cell proliferation. The novelty of this study lies in the method of synthesis as well as engineering nanostructured biomaterials. This is the first study to show generation of multiphased nanostructured biomaterials with cancer controlling properties synthesized via ultrashort pulsed laser interaction. Ultrashort pulsed laser synthesis of functionalized multi-phased nano biomaterial holds immense potential in a myriad of cancer therapy applications such as in implants, diagnostic chips and markers in targeted therapy and opens avenues for the application of various nano forms of silicon in addition to precisely varying number of and composition of phases of both silicon and silicon oxides. 


\section{Chapter 8}

\section{Next Generation bio-functionalized rare phased gradiated gold-silicon/silicon oxide nanostructured hybrid biomaterials to deactivate cancer cell growth}

This chapter introduces a novel nanostructured biomaterial that is a hybrid combination of rare phases of immiscible gold and silicon fabricated via ultrashort pulsed laser synthesis. For the first time, cancer controlling properties of rare phases of gold and silicon that include $\mathrm{Au}_{7} \mathrm{Si}$, $\mathrm{Au}_{5} \mathrm{Si}, \mathrm{Au}_{0.7} \mathrm{Si}_{2.3}$, and $\mathrm{Au} 8 \mathrm{Si} 2$ are reported. The formation and cancer controlling properties of these phases has not been investigated before. Conventionally, pure forms of nano gold and silicon are extensively employed in targeted therapy and drug delivery systems due to their unique properties. However, pure forms of gold and silicon are limited in scope and do not present properties of cancer cell control. Therefore, in this chapter, current trends in cancer therapy are redefined by reporting next generation bio-functionalized, rare phased gradiated gold-silicon oxide nanostructured hybrid biomaterial and its unique cancer controlling properties via ultrashort pulsed laser synthesis. Rare phased gradiated gold-silicon nanostructured hybrids possess inherent drug-like properties that can deactivate the growth of cancer. The nanostructured hybrid is composed of individual gradiated gold-silicon oxide core-shell like nanoparticles forming a three dimensional nanostructure. This nanostructured hybrid biomaterial is homogenous with a gold core enveloped with an increasing gradient of silicon oxide. The cancer controlling properties of rare phased nanostructured hybrids are activated by controlling the ultrashort pulsed laser-material interaction that allows precise tuning of number of phases as well as composition of phases in each individual gradiated gold-silicon nanoparticle in the nanostructured hybrid. In addition to such chemical properties, physical properties such as three dimensionality and density of the nanostructured hybrid and size of individual gradiated goldsilicon oxide core-shell like nanoparticles can be tuned. It is observed that the combination of these unique phases in a gradiated form has unique cancer controlling properties. The interaction of the nanostructured hybrids with cervical cancer cells showed a $96 \%$ or 30 fold reduction in 24 
hours. Cell health was observed to deteriorate on the gradiated gold-silicon oxide core-shell like nanoparticles hybrids with decrease in cell size, nucleus size and number of filopodia. The rate of cancer proliferation and adhesion is controlled by means of encapsulation and passive ingestion of the nanostructured hybrids. This engineered gradiated core-shell like nanostructured hybrid biomaterial presents significant potential due to the combination of gold and silicon in varying phases in a homogenized fashion and satiates the current vacuum in cancer therapy with biomaterials that possess inherent drug-like properties.

\subsection{Introduction}

In recent years, the combination of one or more nano elements to combat multiple functions in cancer treatments has been perceived. Sailor and Park reviewed the combination of multiple elements in a core and shell manner and its advantages and applications [212]. It is proposed that the combination of two elements can further increase an advantageous property or add several advantageous properties in cancer therapy. For instance, Sahoo et al. developed mesoporous silica-coated manganese ferrite nanoparticles that perform as drug delivery carriers as well as MRI contrast agents [213]. The mode of synthesis consists of multiple steps and combined both chemical and thermal methods. Using chemical methods, Li et al. generated gold coated iron oxide nano rose-like structure that has multiple functions such as cancer cell targeting, magnetic resonance and optical imaging [214]. Similar to multiple element nanoparticles and nanoshapes, multiple element nanofibers are also sought after. Yan et al. employed electrospinning to fabricate polyvinyl alcohol core and chitosan shell nanofibers [215]. They showed the internalization of ovary cancer cells over a period of eight days. Therefore, it is observed that the introduction of multiple elements can target different avenues of cancer therapy and diagnostics. However, the combination of immiscible elements such as gold and silicon in a fused form has never been reported before.

Silicon has shown tremendous promise in the field of cancer therapy. Milgroom et al. studied the use of mesoporous silica as targeting agents for ultrasound with breast cancer cells [216]. They state that mesoporous silica stays in the body for a period of 4 weeks. This provides a window for targeting cancer cells. Further, Kallinen et al. confirmed the advantages of silicon as a cancer targeting agent [217]. In addition to cancer cell targeting, silicon has also been employed extensively as a drug delivery vehicle. For instance Peng et al. developed and tested silicon 
nanowires as doxorubicin carriers and produced significant improvement in resistant factor [218]. Additionally, silicon has also been a primary candidate for substrate in fabricating diagnostic devices to study cancer in vitro. Thege et al. fabricated a microfluidic chamber to capture circulating cancer cells [219] and Dhanekar et al. have reviewed the versatility of silicon and its applications as a biosensor [220]. Of similar interest in cancer therapy has been gold in micro and nano form. Fratoddi et al. have reviewed gold nanoparticles and their applications as targeting agents. They attribute the interest in nano gold to its high surface to volume ratio and its ability to be easily functionalized to perform targeted therapy [221]. Further, gold has shown to possess the ability to scatter as well as absorb incident photons aiding in photothermal therapies [222]. Owing to similar properties gold has also shown promise as biomarkers [223].Therefore, a combination of gold and silicon is sought after in multiple cancer therapies. For instance Shen et al. fabricated gold nanorods encapsulated by silica [224]. This nanorod is capable of drug delivery as well as phototherapy owing to gold content. In another study, Wang et al. developed gold nanoparticles coated with mesoporous silica for ultrasound imaging and tumor cell ablation [225]. It is observed that in all studies the combination of gold and silicon has been used a drug delivery carrier but never a cancer controlling agent on its own. Further, these studies show nanostructures of gold and silicon which are not in a fused form and are maintained as separate entities in the nanostructure. Therefore, it is seen that a homogenous combination of immiscible gold and silicon in nanoscale with varying phases has not been explored. Further, the cancer controlling properties of such multi-phased nanostructures has not been investigated. A vacuum in homogenous multi-phased nanostructures is due to absence of high pressure and temperatures in other fabrication methods that is required for formation of rare phases.

In this study, a bio-functionalized, rare phased homogenous gradiated gold-silicon oxide nanostructured hybrid biomaterial is synthesized by ultrashort pulsed laser-material interaction (Figure 1). The nanostructured hybrid is composed of individual gradiated gold-silicon oxide core-shell like nanoparticles made up of core-shell like structures. Crystalline gold forms the core and is enveloped by increasing concentrations of silicon oxides. For the first time, rare homogenized phases like $\mathrm{Au}_{7} \mathrm{Si}, \mathrm{Au}_{5} \mathrm{Si}, \mathrm{Au}_{0.7} \mathrm{Si}_{2.3}$, and $\mathrm{Au}_{8} \mathrm{Si}_{2}$ are synthesized due to unique laser-material interaction mechanisms. When the ultrashort pulsed laser interacts with silicon coated with gold substrate, it vaporizes the substrate. A plume is formed around the interaction zone that is composed of gold and silicon species. Mechanisms such as nucleation, coalescence 
and aggregation takes place while interacting with oxygen in ambient air. The number of phases as well as composition of phases in addition to properties like three dimensionality and nanostructured hybrid size critically depends on characteristics of the laser interaction mechanism such as plume temperature, surface temperature and ionization of the species. These novel phases and their unique combination shows deactivates the growth of cancer. In 24 hours, there is a $96 \%$ or 30 fold reduction in the number of cervical cancer cells. Most surviving cells are rounded in nature with small nuclei indicating apoptosis inducing expressions. This study therefore is the inception of a new mode of cancer controlling by multiple homogenous rare phases in a gradiated fashion. This nanostructured hybrid has a variety of other applications owing to the presence of both silicon and gold such as photothermal therapy, biomarkers for imaging.

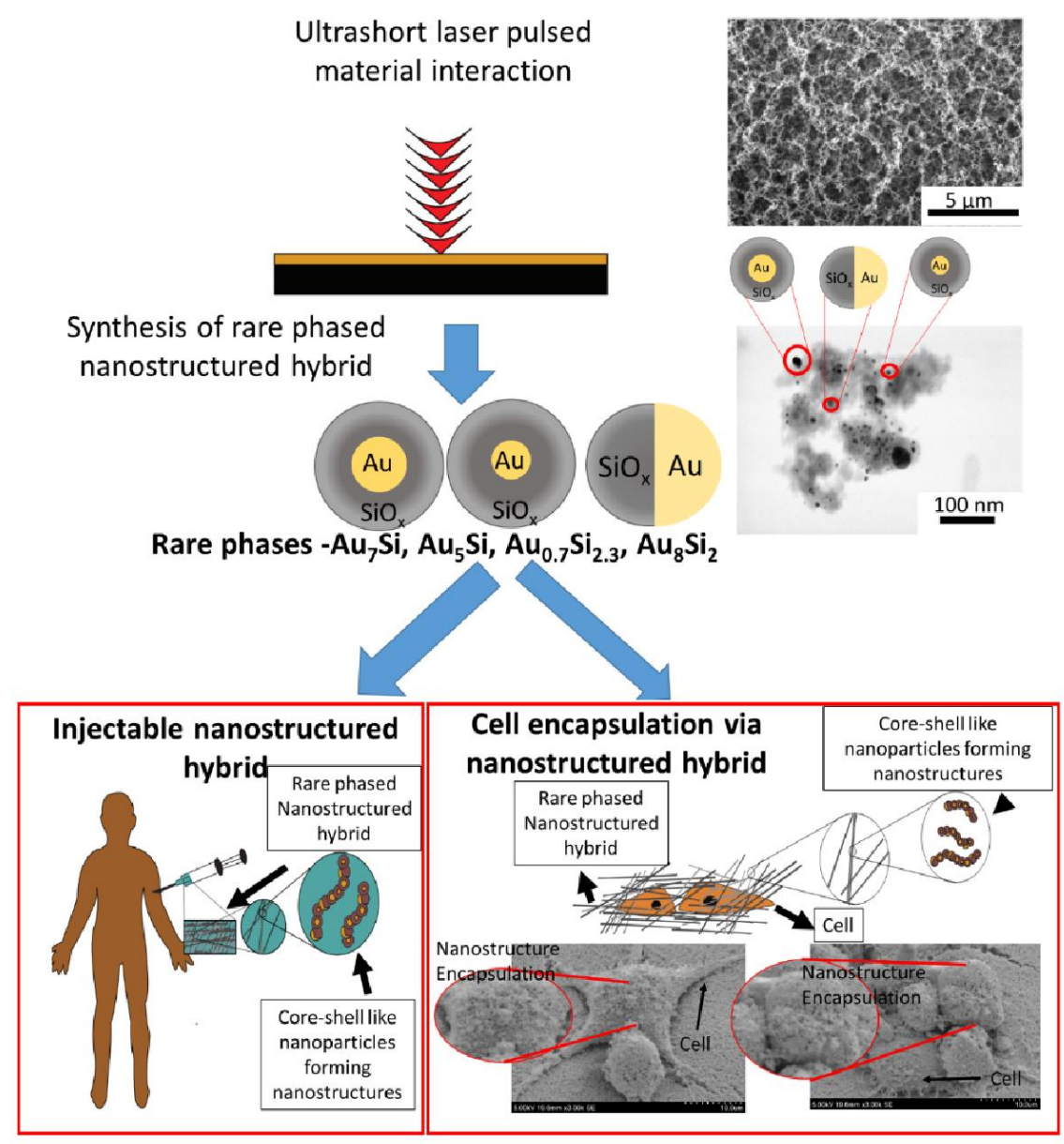


Figure 0-1: Illustration of generation of 'anti-cancer' gold-silicon nano hybrids and its applications

\subsection{Experimental Detail}

\subsubsection{Fabrication of gold-silicon nanohybrid}

Rare phased nano gold-silicon hybrids are directly patterned in a single step using a ultrafast pulsed laser. Undoped silicon wafers $<100>$ with a thickness of $500 \mu \mathrm{m}$ (University Wafers, USA) is coated with gold ( $350 \mathrm{~nm}$ in thickness) and are cut into $2 * 2 \mathrm{~cm}$ samples. These samples are washed with de-ionized (DI) water and ethanol and rinsed in DI water again. These samples are then irradiated using a diode pumped, Yb-doped femtosecond laser system (Clark-MXR Inc. IM-PULSE Series Ultrashort Pulse laser) at $26 \mathrm{MHz}$. The laser irradiates the samples in an array of points at a distance of $50 \mu \mathrm{m}$. The laser material interaction time is maintained at $0.1 \mathrm{~ms}$. The power of the laser determines the percentage of gold in the final 'anti-cancer' nano gold-silicon hybrid structure. Three different powers are used $-16 \mathrm{~W}, 14.5 \mathrm{~W}$ and $12.5 \mathrm{~W} .214 \mathrm{fs}, 714 \mathrm{fs}$ and $1428 \mathrm{fs}$ pulse durations are employed. The laser wavelength is maintained at $1040 \mathrm{~nm}$. All the parameters are controlled by a computer to facilitate high accuracy.

\subsubsection{Characterization of the rare phased gold-silicon nanohybrid}

Scanning electron microscopy (SEM) (Hitachi S 5200) is employed to study the morphology of the three dimensional rare phased nanohybrid. To study its nanoparticle composition and structure, transmission electron microscopy (TEM) (Hitachi H 700 CTEM) is used. X- Ray diffraction (XRD) (Siemens D5000 conventional theta/2theta diffractometer) is used to analyze the material composition/phase of the rare phased nanohybrid. Energy-dispersive X-ray spectroscopy (EDS) is performed to study elemental composition. X-Ray photon spectroscopy (Thermo scientific K-alpha) is conducted to confirm relative quantities of gold, silicon and oxygen.

\subsubsection{Cancer cell-gold-silicon nanohybrid}

The interaction of cervical cancer cells (HeLa, ATCC, USA) with 'anti-cancer' nano hybrid is used to qualitatively and quantitatively study cancer controllability. The cells are grown in DMEM/F12 medium supplemented with $10 \%$ fetal bovine serum and $1 \%$ anti-biotics. The 
hybrids are sterilized under UV light for 20 minutes. Subsequently, they are placed in petri dishes with HeLa cells at a density of $10^{5}$ cells $/ \mathrm{ml}$, totaling $3 \mathrm{ml}$ per dish. The petri dishes are placed in an incubator for 24 and 48 hours.

\subsection{Results and Discussion}

\subsubsection{Fabrication of gold-silicon nanohybrid}

The interaction of ultrafast pulses with the surface of gold coated silicon results in the formation of nanoparticle aggregates that assume a nanofibrous structure (Figure 0-2A). A single laser pulse is not capable of ejecting material from the surface of a metal like gold. In this study, multiple pulses interact with the surface of silicon. The pulse to pulse separation time is an important parameter and is in the nanosecond range. The pulse to pulse separation time determines the heat dissipation between pulses.It is noted that longer pulse to pulse separation times lead to more laser energy that completely depletes the gold from the surface.

When the ultrafast laser pulses strike the surface of gold,multiphoton excitation processes occur. The conduction band electrons absorb the energy thus increasing the electronic temperature. The heat is not transferred to the latticeowing to low heat capacity of electrons in a metal. The temperature at the surface of gold after multiple pulses of laser interaction depends onpulse duration, thermal diffusion coefficient, heat conduction coefficient, reflection coefficient, residual energy coefficient, laser spot diameter and ratio of pulse duration to pulse interval[226]. As the equation indicates the surface temperature is directly proportional to average power of the laser.The average power of the laser also influences the number of atoms that are ejected from the surface [196]. The average power determined the concentration of gold in the nanostructures. Varying plume mechanism determines that formation of gold-silicon nano hybrids.

The synthesis of the gold-silicon nanohybrids occurs due to vapor condensation mechanism. The plume is composed of silicon and gold species. The interaction of the plume with ambient air initiates its cooling. The matter in the plume is in a supercritical fluid state [197]. This commences a cascade of events where ions in the plume collide to form molecules, whichin turn collide due to thermal motion. Subsequent temperature reduction begins nanoparticles formation[198]. The unique property of the gold-silicon nano hybrid is the synthesis of goldcore silicon-shell nanoparticles. The size of the gold-core can be manipulated based on the average power of the ultrafast laser pulses. At a lower power, smaller gold cores are observed.At 
higher power, the temperature of the plasma itself can cause the surface to heat hence ejecting larger particles into the plume[227]. Therefore at higher power, it is observed that the majority of gold cores are small but a small percentage of gold cores are bigger encompassing almost the entire shell (Figure 0-2B).

Ultrafast pulsed laser interaction also produced varying density of gold-silicon hybrids (Figure 0-2 (C-F)). The zone of pulsed laser material interaction has the highest quantity of gold-silicon nano hybrids. Away from the zone of interaction, the gold-silicon nano hybrids gradually reduce. This study will also study the influence of density of nano hybrids on cell growth.

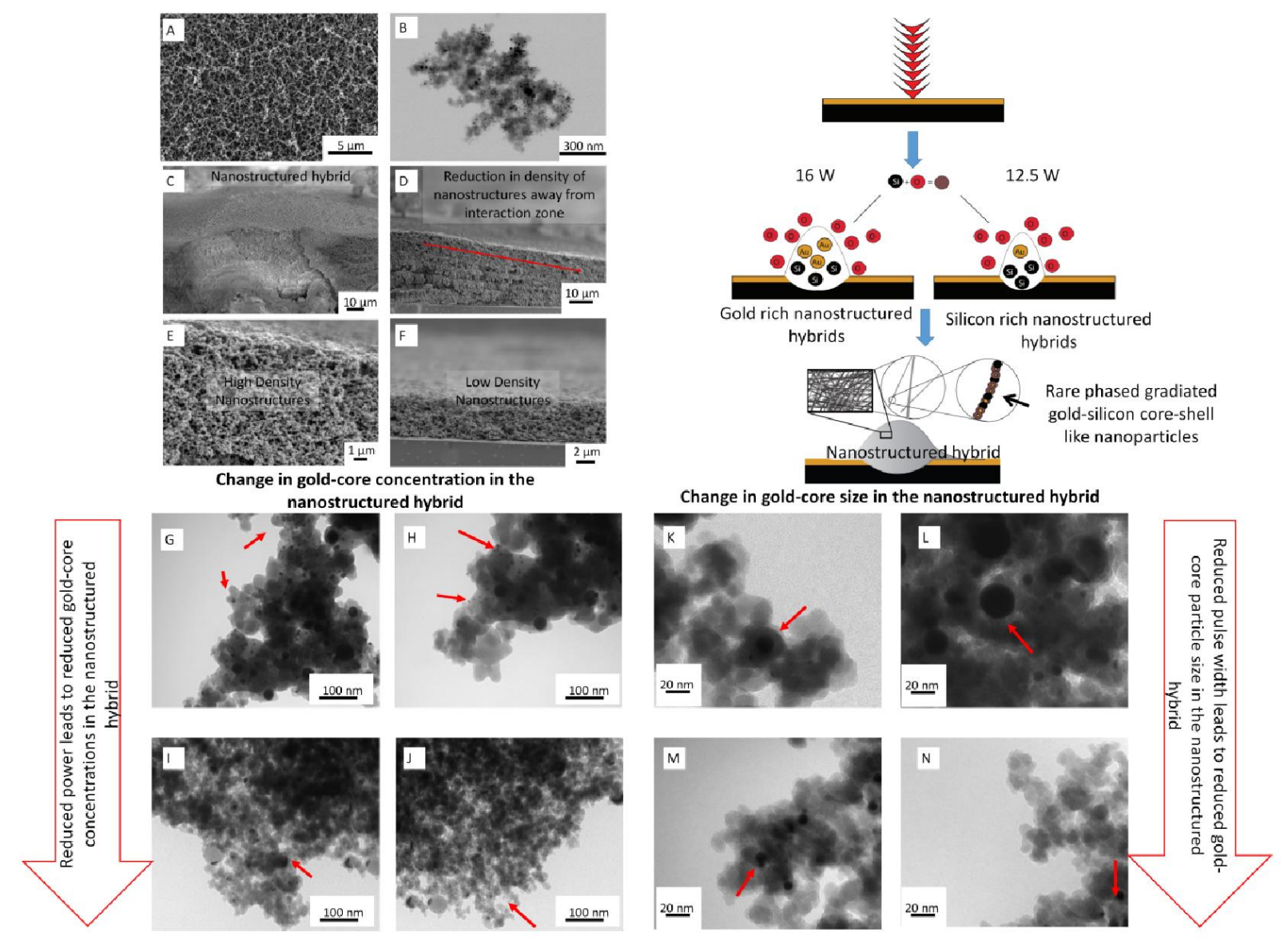

Figure 0-2: (A) SEM of gold-silicon nano hybrids (B) TEM showing gold-core silicon-shell structure (C-D) Varying densities of nano hybrids (E) High density nano hybrids (F) Low density nano hybrids. The figure also illustrates the varying concentration of gold in the nano hybrids (G-H) High gold concentration (I-J) Low gold concentration (K-L) Large gold-cores (M-N) Smaller gold-cores. 


\subsubsection{Compositional analysis of gold-silicon nanohybrid}

The composition of the 'anti-cancer' gold-silicon nano hybrids is analyzed by employing EDX, XRD and XPS. EDX illustrates the presence of oxygen, gold and silicon in the gold-silicon nano hybrids (Figure 0-3(A-D)). As Figure 0-3A indicates, the gold forms the core of the silicon shell structure, the dark spots indicating gold cores. The shell like structure around gold is made up of silicon oxides in amorphous form. EDX indicates the silicon and oxygen peaks are always proportional indicating vapor condensation formation mechanism. The formation of a gold core can be attributed to the diffusion of gold through silicon [228]. Subsequent to the formation of core, more silicon is attracted towards this structure giving rise to a shelled silicon structure around gold. Further, it is noted that amorphous silicon will allow more diffusion of gold. To further quantify the gold-silicon nano hybrids, XPS was conducted. XPS shows that the presence of oxygen increases with an increase in average laser power. This increase in oxygen can be attributed to higher atomic ratio of oxygen to silicon and gold, or a larger surface area of the gold-silicon nano hybrids [229]. The XPS also points to an increase in gold concentration with an increase in average laser power. To confirm these results XRD is performed (Figure 0-3E). The XRD confirmed an increase in gold concentration at higher laser powers (Supplementary Information (S2)). XRD analysis is also performed on control samples which are silicon coated with a small layer of gold without any modifications. The control virgin gold samples reveal crystalline gold in (111), (200) and (220) orientations. XRD on both high power laser interaction and low power laser interaction showed presence of crystalline silicon in (111), (220) and (311) orientation. In addition, at lower laser power interaction, the formation of gold silicides is observed. The possible candidates based on this peak are- $\mathrm{Au}_{7} \mathrm{Si}, \mathrm{Au}_{5} \mathrm{Si}, \mathrm{Au}_{0.7} \mathrm{Si}_{2.3}, \mathrm{Au}_{8} \mathrm{Si}_{2}$. Further, the presence of amorphous silicon and silicon oxide is observed at both laser power interactions. Quantification of the gold and silicon in the gold-silicon nano hybrids was obtained using Rietveld refinement. The quantitative analysis proves that the concentration of silicon does not change with power. An increase in power only increased the concentration of gold. Similar to oxygen concentration, gold may also show an increase at higher laser powers owing to higher density of gold-silicon nano hybrids. Higher density of fibers would require additional species in the plume. It is hypothesized that the depth of ablation is similar at all powers, however, the width of ablation is higher for higher power and therefore there is a consequent increase in gold 
in the plume and hence in the nano hybrid. Additionally, the XRD shows an increase in amorphous silicon/silicon oxides at lower laser powers. Another aspect observed in the nano hybrid is that only silicon forms oxides. This is due to similarity in the weight of the atom. Since oxygen and silicon are similar compared to gold, it is more likely that silicon forms oxides[59].

Based on the characterization method, a mechanism of formation is proposed. When the laser strikes the surface of silicon coated with a thin layer of gold, the surface is ablated and gold and silicon species are ejected into a plume. The power of the laser determines the width of laser ablation and the pulse duration determines the depth of ablation. The plume starts expanding and species begin mingling with ambient air. Monomer formation is initiated followed by coagulation and coalescence. Once coalescence stops aggregate formation takes place. In the plume, gold diffuses through silicon to form a gold-core silicon-shell structure. Individual silicon nanoparticles are also formed. The formation of gold silicides is attributed to gold alloying with silicon at the surface itself. The initial pulses let gold diffuse through the silicon substrate alloying it.
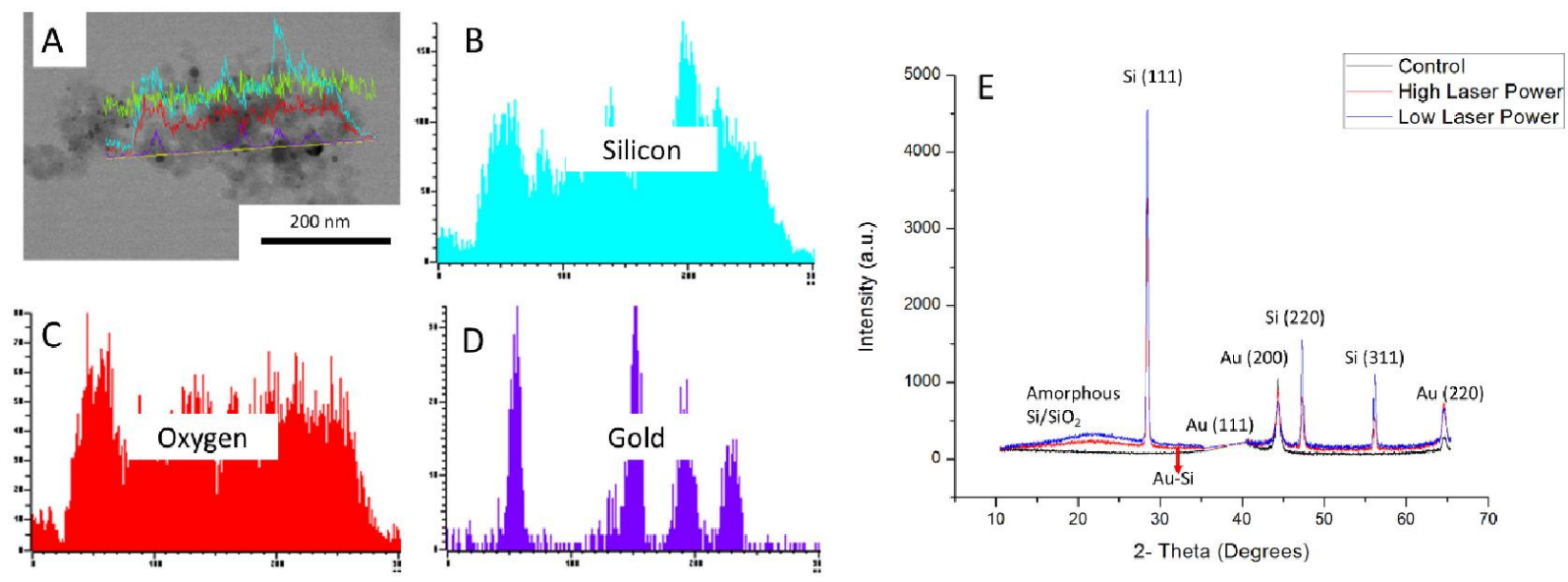

Figure 0-3: (A-D) TEM and EDX of gold-silicon nano hybrids (E) XRD micrograph of goldsilicon nano hybrids.

\subsubsection{Manipulation of cancer}

\subsubsection{Role of density of gold-silicon nanohybrid}

The high density nano hybrids adhere strongly to the surface of the substrate owing to its high temperature synthesis. Their formation is directly on the surface of the substrate. The high 
temperature synthesis causes strong bonding between the nano hybrids. The nano hybrids resemble a three dimensional matrix that is interlinked and randomly oriented with varying porosity. While other research studies have proven the proliferative nature of such a matrix, this paper discusses cancer cell manipulation and eventual cell death. Figure 0-4(A-D) illustrates the influence of density of nano hybrids on the growth of cells. A reduction of $97 \%$ and $96 \%$ in cell counts is seen at low and medium densities respectively. The high density nano hybrids showed an $85 \%$ decreasein cell count. Therefore, it is observed that nano hybrids do severely reduce the number of cells. The high density nano hybrids strongly adhere to each other as well as the substrate. The medium and low density nano hybrids are deposited nano hybrids. Therefore, their adherence to the substrate is weak. This enables its movement in cell medium. The strong nano hybrids encapsulate cells whereas the medium and low density nano hybrids reduce cell growth by inducing passive cell endocytosis (Figure 0-4E). These processes lead to the presence of three kinds of cells- Round cells, small and flat cells (under $200 \mu \mathrm{m}$ in area) and big flat cells (over $200 \mu \mathrm{m}$ in area). Figure 0-5 shows the relative occurrence of the three types of cells. Combining the results, it is evident that the majority of cells that are found on the high density nano hybrids are rounded cells. Rounded cells or shrunken cells display more apopotic tendencies [230].

\subsubsection{Role of gold concentration in gold-silicon nano hybrids}

The synthesis of the gold-silicon nanohybrids was varied based on the concentration of gold required. Silicon rich or gold rich nano hybrids are synthesized based on the power of the laser. An increase in power proportionally increased the concentration of gold in the nano hybrids. Figure 0-6Aillustrates an interesting aspect of cell growth over 24 and 48 hours. At 24 hours, it is observed that the gold rich nano hybrid presented with increased cell numbers. However, at 48

hours, the cell numbers are completely inverted and the cells gold rich nano hybrids dramatically decrease. This unique tendency is owing to two separate but parallel mechanisms. At 24 hours, the gold rich nano hybrids form a biocompatible substrate for the growth of cells. This increases the number of cells in relation to silicon rich nano hybrids. However, when compared to control virgin gold, the percentage of cells is still less due to the inherent non compatible nature of the three dimensional structure of the nano hybrid. 

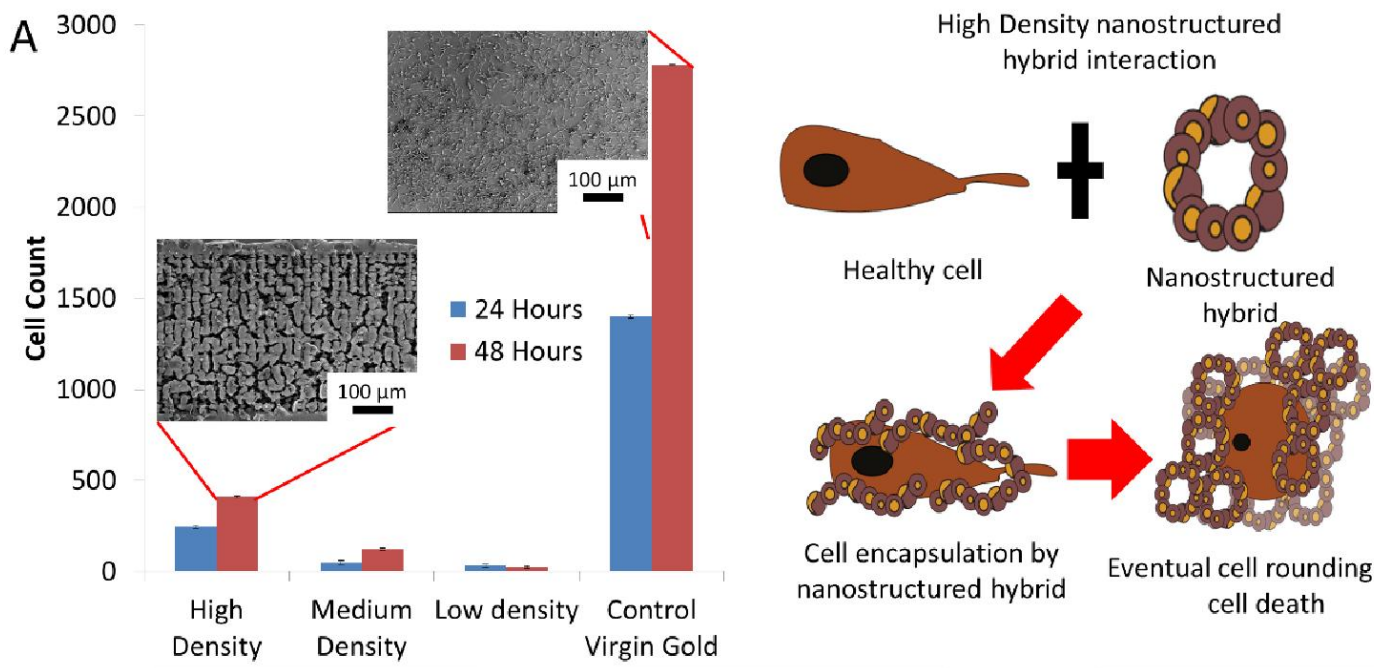

Cell encapsulation by nanostructured hybrid

Eventual cell rounding and cell death
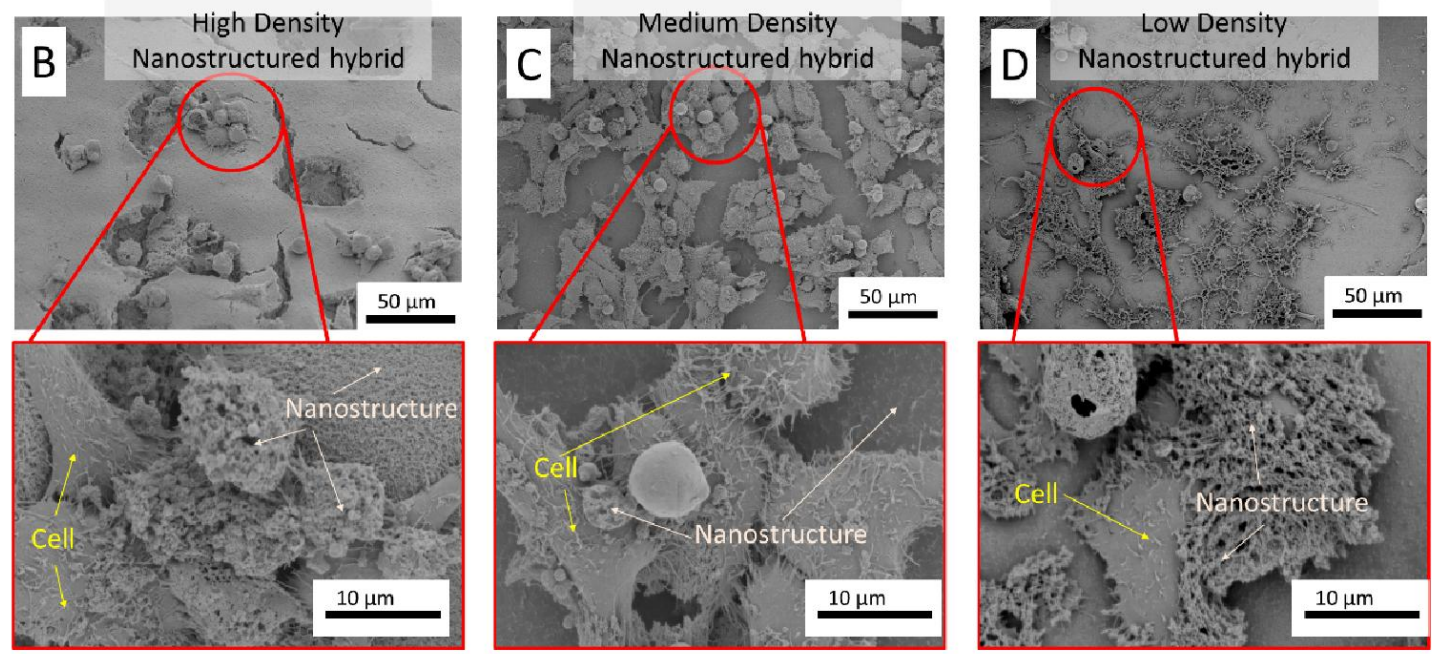

Figure 0-4: (A) Graphical representation of cell growth on various densities. Cell growth on (B) High density (C) Medium Density (D) Low Density (E) Illustration of nano hybrid interaction with cell. 


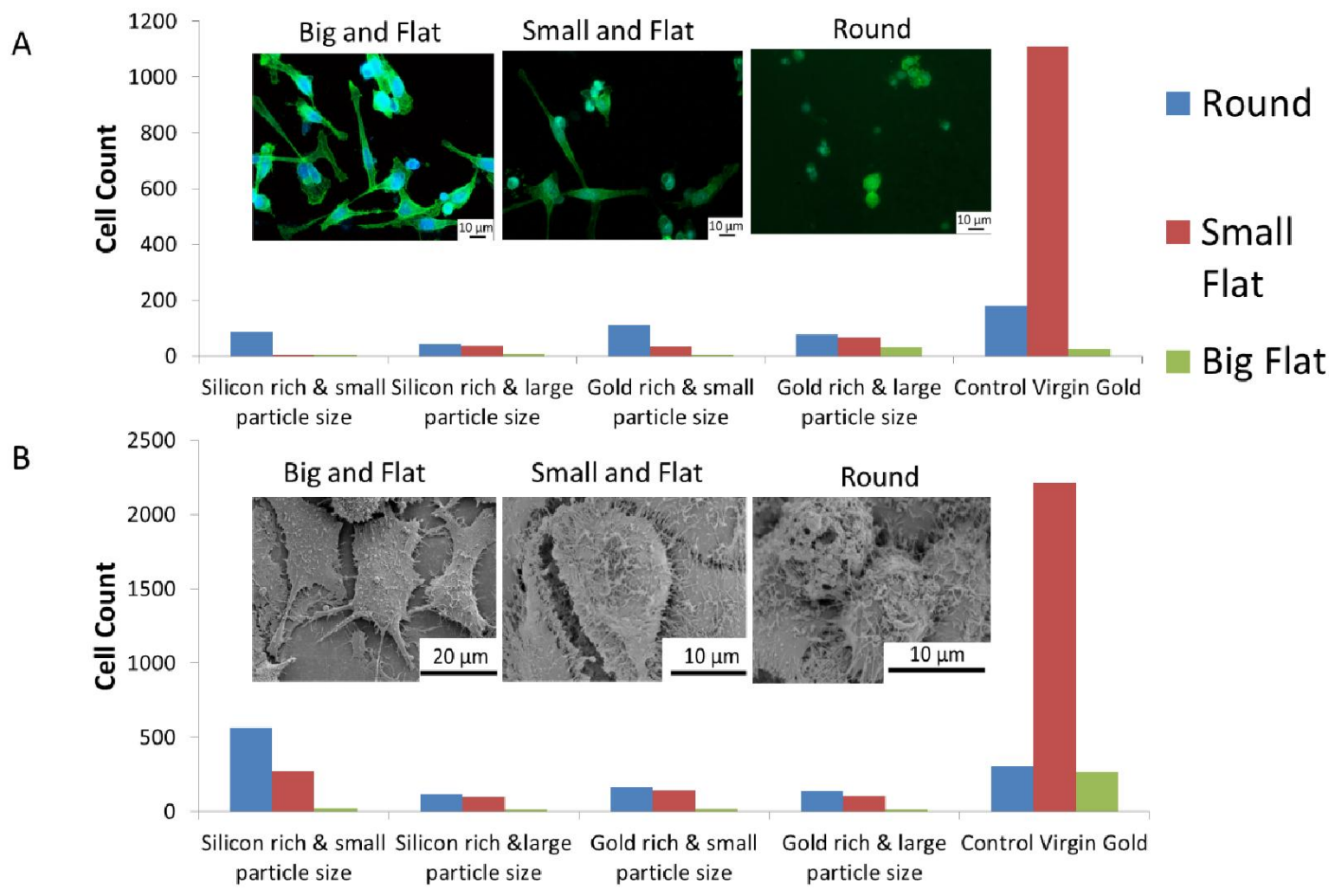

Figure 0-5: Relative occurrence of different kinds of cells at (A) 24 hours and at (B) 48 hours.

Therefore, it is seen that while the gold rich nano hybrids present surface for growth, they do not enhance the growth of cells[231]. At 48 hours, a complete reversal of cell growth takes place. The silicon rich nano hybrids present significantly higher number of cells than gold rich nano hybrids. This could be attributed to the process of endocytosis. The cells may ingest the goldcore silicon-shell nanoparticles which causes cell death (Figure 0-6(B1-B3, C1-C3)) [208]. An interesting point of observation is that cells decreased only with increased gold in nanostructure form. When control virgin gold is studied manifold increase in the number of cells is observed. Majority of the studies involving gold have looked at the biocompatibility of gold [236, 237]. However, this study shows a marked decrease in cells with increasing concentrations of gold. This decrease is attributed to the properties of the size of the gold nanoparticles. 


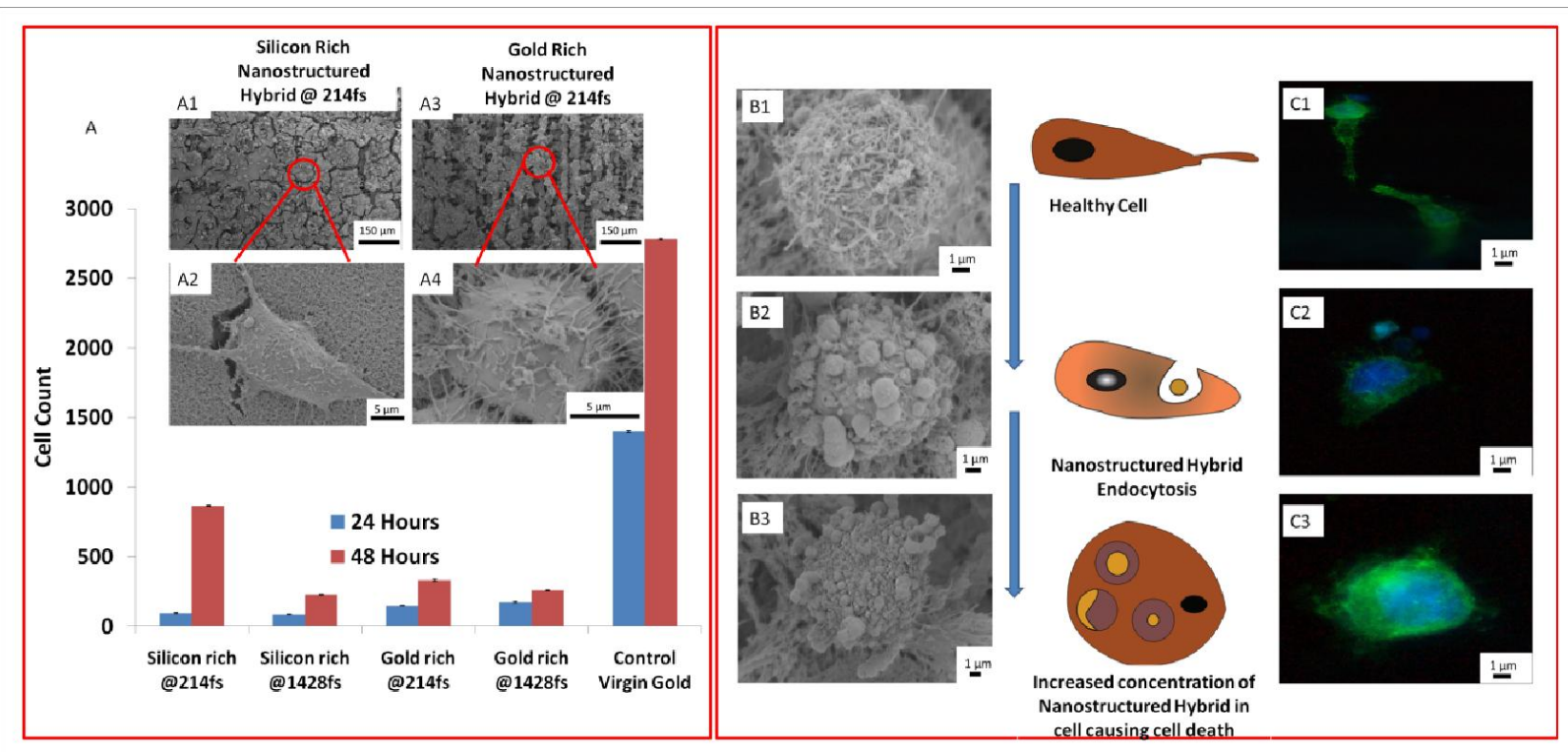

Figure 0-6: (A) Graphical representation of cell interaction at different concentration of gold. (B1-B3 and C1-C3) Representation of endocytosis of nanoparticles in nano hybrids and eventual cell death.

\subsubsection{Role of particle size in gold-silicon nanohybrid}

The particle size of the nano hybrids is varied based on the laser pulse duration. At shorter pulse durations, the temperature of the plume is significantly higher. Consequently, the competition between nucleation and particle growth rate causes smaller sized nanoparticles to form at shorter pulse durations[91]. Therefore, at each density and at each gold concentration, there exists two particle sizes. Shorter pulse durations, 214 fs causes smaller nanoparticles size and longer pulse duration, 1428fs causes larger particle size. As a result, the cell growth patterns are also different.Figure 0-7 presents the growth of cells at 24 and 48 hours and the difference in growth between shorter and longer pulse width. Consistently, shorter pulse widths show more cell numbers compared to longer pulse widths. Shorter pulse widths points to small nanoparticles and longer pulse width to larger particles. It has been proven that smaller nanoscale materials enhance the growth of cells [206, 238]. 


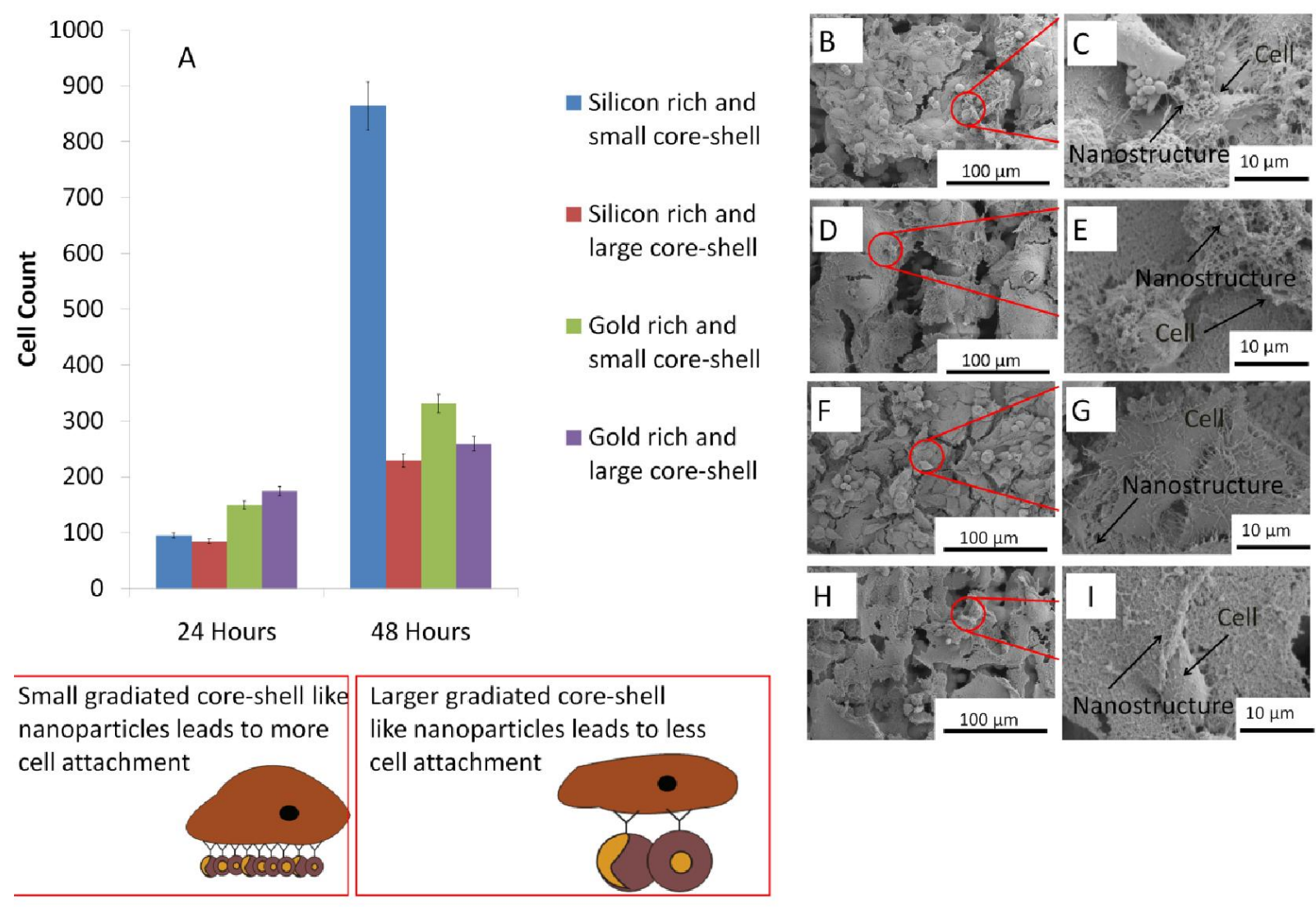

Figure 0-7: (A) Interaction of cells at different pulse widths (B-C) Gold rich at 214fs (D-E) Gold rich at $1428 \mathrm{fs}(\mathrm{F}-\mathrm{G})$ Silicon rich at 214fs (H-I) Silicon rich at 1428fs.

Therefore, it is observed that multiple parameters on the gold-silicon nano hybrids effect the growth of cells. In all parameters, there is also a change in the quantity of nano hybrids being generated.Higherpower, shorter pulse width also causes an increase in nano hybrid quantity. Nisbet et al. presented a correlation between densities of nanofibers and cell growth[202].Further Zhao et al showed that nanostructures initiate more collagen secretion and protein adsorption [207]. The nano hybrids generated in this study are made up nanoparticles forming a nanofibrous structure. Therefore, at 24 hours this increased area cause initial attachment of cells. At 48 hours, even though cell proliferation is observed, it is less compared to the control virgin gold substrates. Further, higher gold content also resulted in reduction of proliferation. It is postulated that after initial attachment, cells either get encapsulated in the nano hybrid or are apoptotic due to passive nanoparticles ingestion. It has previously been stated that cancer cells migrate through fibers to metastasize at different areas in fibers[204]. Similar trends are observed here during 
encapsulation. Initial attachment of cells on gold rich nano hybrids may also be attributed to their predominantly crystalline phase (Figure 0-3E) as shown previously [205].

Further characterization of the gold-silicon nano hybrids is performed based on fluorescence microscopy. Control virgin gold showed random growth of cells in all directions. The cells grow in a flattened manner with stretched cytoskeleton. Control samples also show bright focal adhesion points and stress fibers. Stress fibers communicate surface properties to the cells [174]. Therefore, cells grown on gold-silicon nano hybrids showed remarkably different properties compared to control samples. The actin cytoskeleton of cells on nano hybrids shows a plain surface with no stress fibers which may be an indication of apoptosis. As the cells move to lower densities of nano hybrids, they regain their healthy structure.

\subsubsection{Exploring cell health of gold-silicon nanohybrid}

\subsection{Nucleus Study}

It is observed that the nucleus does not align itself to the fibers but grows in a random fashion. However, the shape and size of the nucleus depends on the density of the nanohybrids (Figure $0-8)$. The nucleus of cells in contact with the nano hybrids are elliptical owing to cytoskeletal forces [175]. The gold rich nano hybrids present nuclei that span the entire range of the cells, suggesting restricted expansion of nuclear volume [235]. It has been proposed that the size of the nucleus triggers signaling in the cells which may lead to apoptosis. The reduced size of the nucleus on gold rich and short pulse synthesized nanohybrids may point to a similar conclusion. The nanohybrids synthesized at higher powers may also lead higher porosity with concurrent smaller pores. Cells on the surface of such nanohybrids may show smaller area of the nucleus and cell to navigate through the pores as it is seen that decreased nucleus area alters rigidity of the cell[204]. Further, Webster et al. have proposed that altered nuclear shape maybe an indication of changed chromatin reorganization and possible gene expression changes[209]. This

altered gene expression might be a potential reason for a large number of rounded cells on gold rich nanohybrids. 

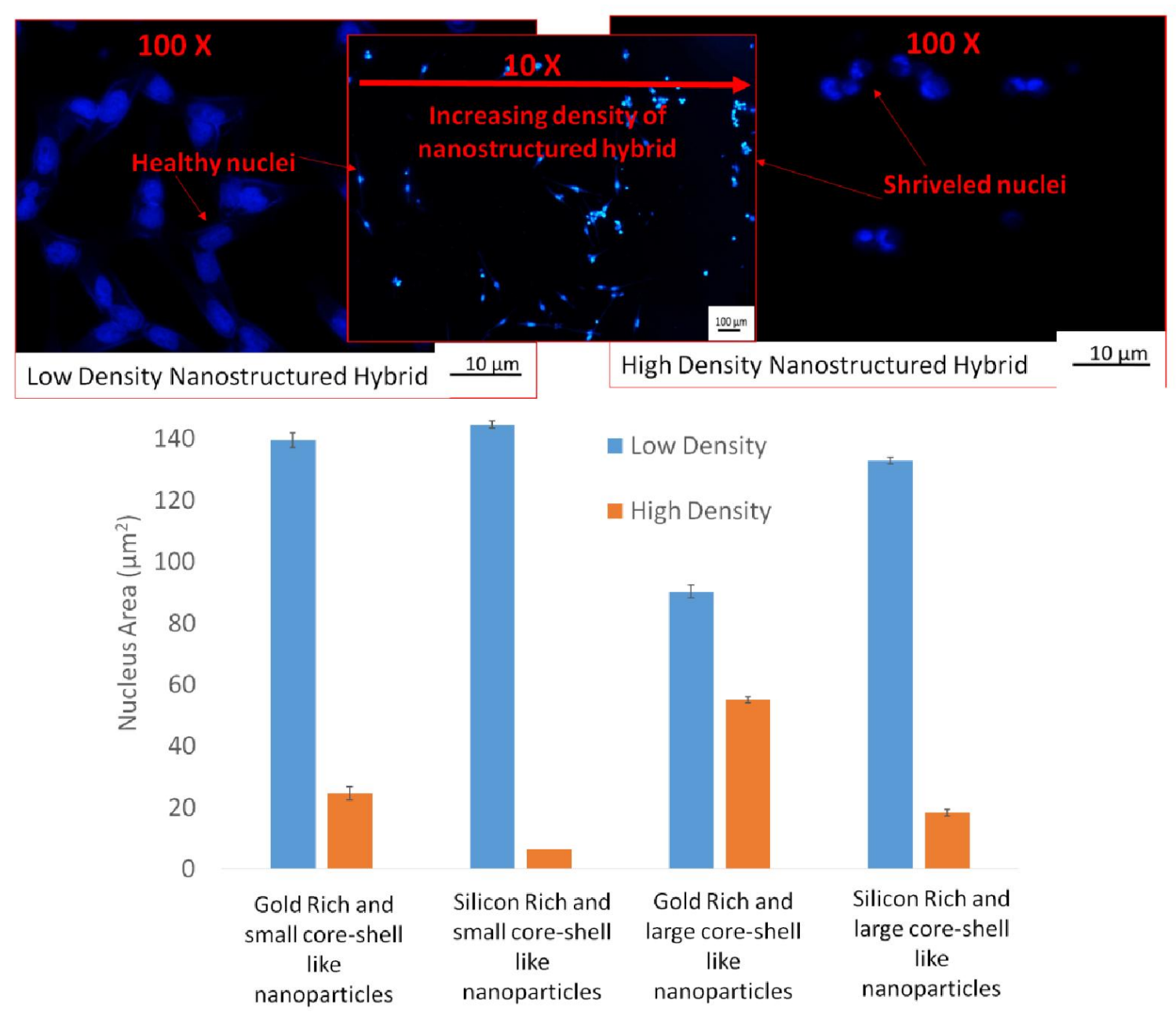

Figure 0-8: Nucleus area illustrating cell health. Short pulse presents smaller nucleus sizes indicating inadequate cell health.

\subsection{Filopodia Study}

The filopodia of the HeLa cells was studied via SEM to analyze migration properties of the cells on high and low density nanohybrids as well as control virgin gold (Figure 0-9). It is observed that the filopodia on virgin gold grow randomly in large numbers. Cells growing on high density nanohybrids showed a drastic reduction in the number of filopodia. The filopodia of cells growing on low density nanohybrids showed on average lesser filopodia than virgin gold. This may point to low density nanohybrids presenting cells with nano contact points that favor filopodial growth. It is known that cells require to spread in order to stratify various phases of 
cell growth [236]. Filopodia formation is an integral part of this cell growth. Therefore, while normal growth of cells is observed on control virgin gold, the absence of similar number of filopodia points to a stunted cell growth on nanohybrids.

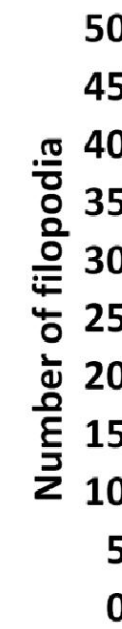
High Denisty
Low Desnity
Control virgin gold
Nanostructured Hybrid Nanostructured Hybrid

Red arrows indicate filopodia and nanostructured
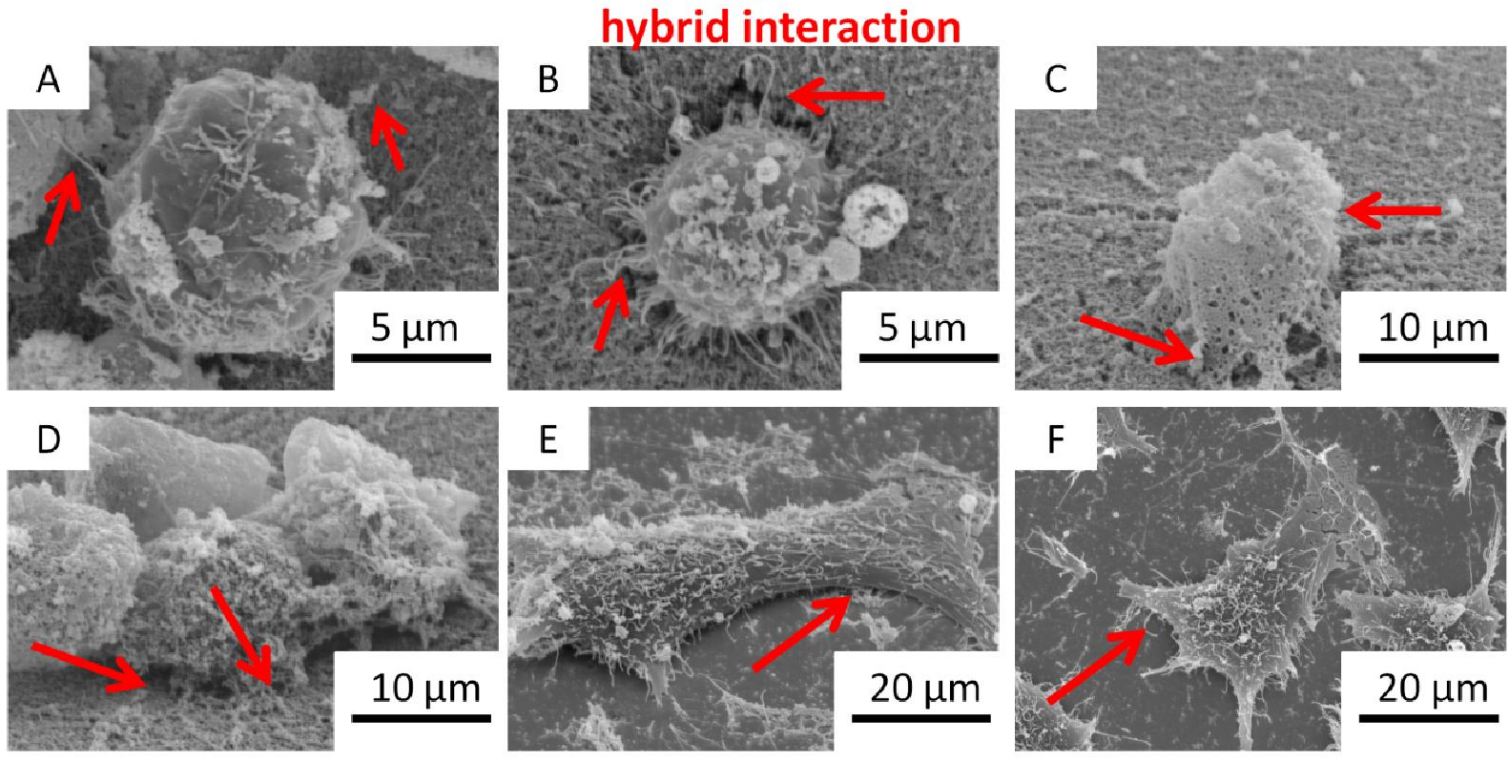

Figure 0-9: Graph depicts number of filopodia on various nanohybrids and control virgin silicon. (A-B) High density nanohybrids (C-D) Low Density nanohybrids (E-F) Control virgin gold. 


\subsection{Summary}

Gold and silicon have shown tremendous potential for applications in cancer therapy owing to their biocompatibility, electrical and thermal properties. However, majority of research with these elements has been in cancer marker detection, photodynamic therapy and potential for drug delivery carriers. To the best of our knowledge, there have been no studies that have explored the possibility of combining silicon and gold and applying them as cancer controlling agents. In this chapter, a bio-functionalized rare phased nanohybrid of gold and silicon in core-shell like form is introduced. The generation of new rare phases of gold-silicon such as $\mathrm{Au}_{7} \mathrm{Si}, \mathrm{Au}_{5} \mathrm{Si}, \mathrm{Au}_{0.7} \mathrm{Si}_{2.3}$, $\mathrm{Au}_{8} \mathrm{Si}_{2}$ are reported.This bio-functionalized nanohybrid is fabricated by ultrashort pulsed laser synthesis with pulse to pulse separation of micro to nanoseconds. The precise tunability of the laser enables the modification of singular core-shell like structures in their phase and size. In addition, the concentration of these phases can also be modified. These parameters of the biofunctionalized nanohybrid depend on the ultrashort pulsed laser-material interaction that can be altered based on surface and plume temperature. This bio-functionalized nanohybrid has significant cancer controlling properties. The interaction of cervical cancer cells with the goldsilicon nano hybrids showed a $96 \%$ reduction in the proliferation of cancer cells in 24 hours. The reduction in cell proliferation is attributed to concentration of gold, particle size and density of nano hybrids. The mode of cell proliferation reduction is ascribed to two theories- encapsulation by the nano hybrid and passive ingestion of the gold-silicon core shell. This bio-functionalized gold-silicon nanohybrid presents a new perspective on cancer therapy. This method not only reduces the proliferation of cancer cells but can also act as drug delivery vehicles and biomarkers. 


\section{Chapter 9}

\section{Summary and Future Research}

\subsection{Summary}

In this dissertation the fabrication of biomaterials is presented using ultrashort pulsed laser synthesis. Notably, both cell proliferation and cell control/manipulation is achieved of silicon substrates employing this unique technique.

The main objective of this thesis was to present laser assisted synthesis of biomaterials as a viable option. Cell proliferating and cell controlling substrates were sought after.

The fabrication of a three dimensional, interwoven, interlinked, randomly oriented nanofibrous matrix is presented. The growth of fibroblasts showed a $300 \%$ increase in proliferation. Characterization studies revealed high zeta potentials and high adhesion forces. Degradation studies showed very low degradation of the nanofibrous structure over period of 1 week. The growth of osteoblasts was also studied on the nanofirbous matrix. It is observed that osteoblasts and fibroblasts proliferated well on the matrix albeit with different adhesion properties.

The possible control of cell proliferation was envisioned and hence gold was added to the nanofibrous matrix. Gold was previously thought to be a biocompatible element. However, the addition of gold to the nanofibrous matrix resulted in less adhesion of cells to the nanofibrous matrix. An increase in concentration of gold caused the cell proliferation to decrease. Further, characterization studies showed decrease in zeta potentials and force of adhesion for increased gold concentrations, supporting cell interaction results.

Therefore, a cell proliferative surface was successfully fabricated. Subsequently, the synthesis of cell controlling/manipulative zones using laser assisted synthesis was investigated.

Sub-surface modifications to silicon were performed without surface damage. New phases of silicon and silicon oxides were created. Remarkably, conventionally unstable phases such as stishovite were found to be stable after sub-surface modifications aiding in cell control. The control of cancer was studied using sub-surface modified silicon was successfully achieved. The migration, cytoskeleton and proliferation rate of cancer cells was precisely controlled. 
In an effort to not only reduce rate of proliferation of cancer but also induce apoptosis, the use of nanostructures was envisioned. Conventionally, nanostructures have been used as cell proliferative surface. In this dissertation nanostructures have been used to achieve controlled apoptosis of cancer cells. The rate of controlled apoptosis was increased with the addition of gold to the nanostructured matrix.

The main contribution of this dissertation can be highlighted as follows:

Ultrashort pulsed laser synthesis of biomaterials in two of its chief areas- cell proliferation and cell control and manipulation is achieved; using either changes to the topography or generating sub-surface modifications without surface damage.

A three dimensional, interlinked, randomly oriented natural ECM-like nanofibrous matrix is fabricated; for cell proliferation applications. Fibroblasts showed a $300 \%$ increase in cell proliferation on this ECM-like matrix. Characterization proved this is a promising candidate for tissue engineering applications. The nanofibrous matrix also enhanced the proliferation of osteoblasts proving it is a versatile candidate with multiple applications.

Cell controlling surfaces fabricated by sub-surface modification and no surface damage; cell isolation, cell repelling and single cell trapping is also observed. Further, similar results are obtained with cervical cancer cells. The sub-surface modified silicon is able to precisely control the growth patterns of cancer cells. This has potentials applications in the MEMS/NEMS and diagnostics field.

Nanostructured controlled apoptosis of cancer; via passive endocytosis. The cells passively ingest the nanoparticles causing cell death. The rate of apoptosis is increased with the addition of fold nanoparticles. 


\subsection{Future Research}

\subsubsection{Gold/Gold-Palladium enveloped $\mathrm{Si} / \mathrm{SiO}_{2}$ 'anti-cancer' nano therapeutic agents}

\subsubsection{Introduction}

A novel method of controlling the growth of cancer cells using a three dimensional nanofibrous network that is interlinked and is composed of $\mathrm{Si} / \mathrm{SiO}_{2}$ nanoparticles is introduced with the addition of sputtered gold or gold-palladium (Figure 9-1). Preliminary results indicate influence of density of nanofibers, concentration of gold and gold-palladium and the mode of laser synthesis on the growth of breast cancer cells.

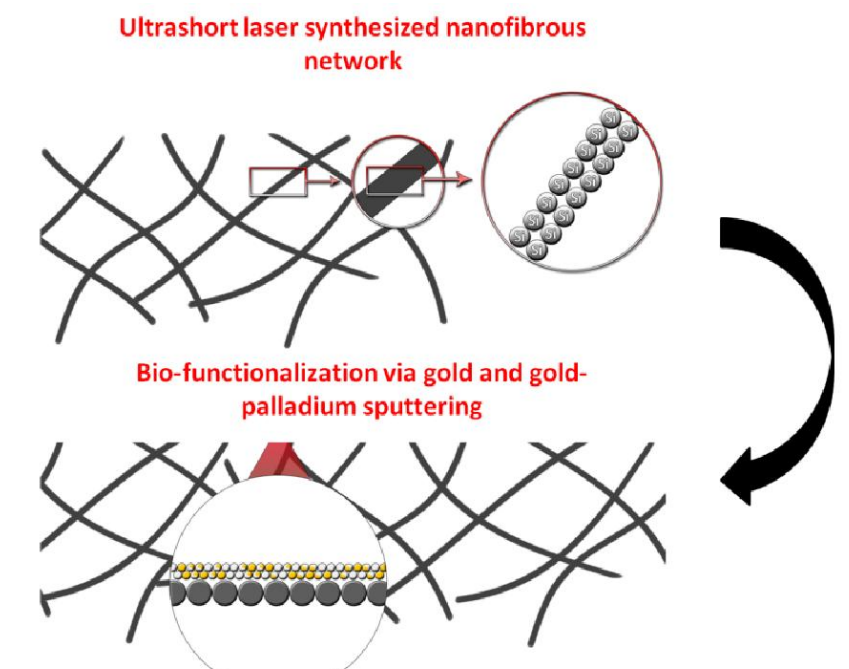

Figure 9-1: Illustration of silicon nanofibers enveloped by gold or gold-palladium

\subsubsection{Preliminary Results}

The interaction of functionalized silicon nanofibers with breast cancer cells reveals remarkable manipulative and controlling properties of the nanofibers. It is observed that the density of nanofibers plays an important role in the control of cell growth. Figure 9-2 presents the influence of gold and gold-palladium both at high density and low density on cell growth. It is hypothesized that the high density nanofibers encapsulate the cells and prevent its growth as shown in the illustration inFigure 9-2. While cells grown on low density nanofibers do show more cell growth compared to high density nanofibers, they are still lower than cells grown on 
control/ virgin silicon. Results show a $99.5 \%$ decrease in cell growth on high density nanofibers compared to virgin silicon.

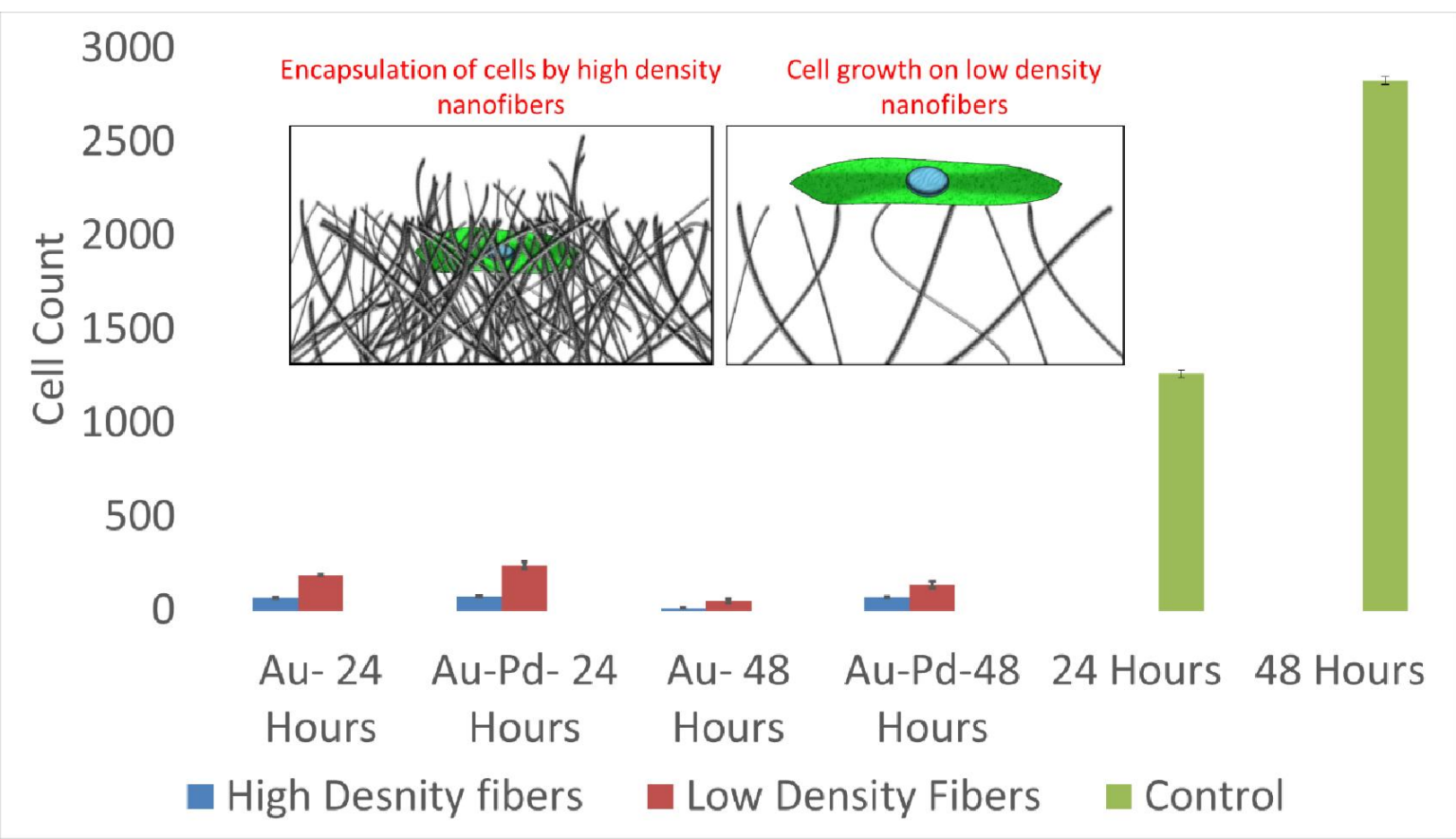

Figure 9-2: Influence of gold and gold-palladium and density of nanofibers over 24 and 48 hours.

Both gold and gold-palladium showed between $65-70 \%$ of reduction in the number of cells on high density of nanofibers. This is contrary to previous research that has shown an increase in cell proliferation with gold and gold-palladium coatings [237], [238]. The reason for this might lie in the size of gold and gold-palladium. Research has shown that nanoscale particles can enter a cell via endocytosis and hence prove to be cytotoxic [110]. 


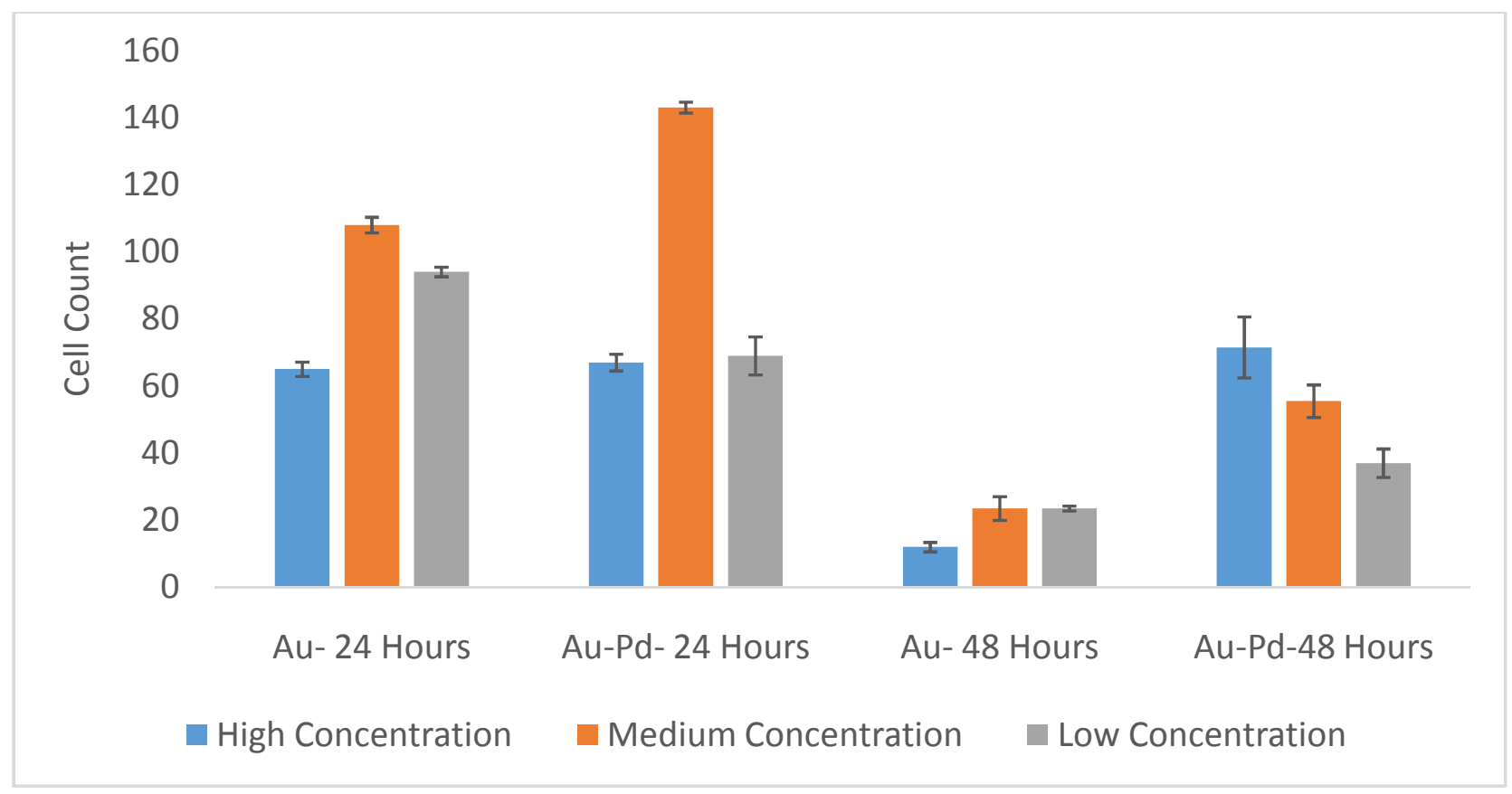

Figure 9-3: The influence of concentration of gold and gold-palladium on cell growth.

Concentration of gold and gold-palladium regulates the growth of breast cancer cells (Figure 9-3). It is seen that the material chemistry plays an important role in cell growth. At 24 hours, both gold and gold-palladium showed similar cell growth. However, at 48 hours there was a marked difference in cell growth between gold and gold-palladium. It is observed that gold at 48 hours, prevented cell growth to a greater degree (Figure 9-4). This may be due to a difference in the internalization of gold and gold-palladium. 


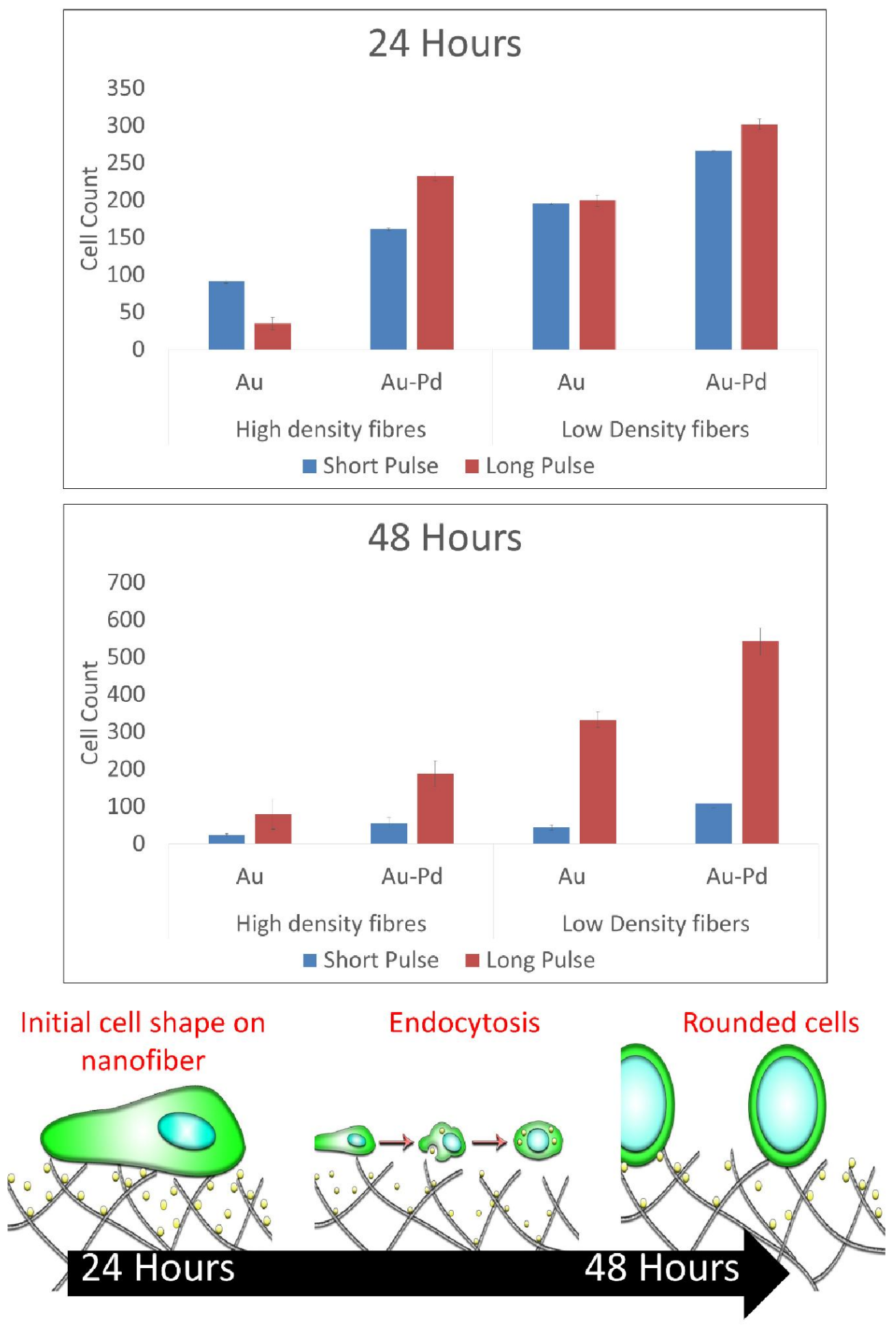

Figure 9-4: Growth of breast cancer cells on gold and gold-palladium enveloped silicon nanofibers at 24 and 48 hours fabricated at short and long pulse. 
Further research in this regard is required to explore the material characterization and its consequent effects on cells growth.

\subsubsection{Selective biomimicking surface nano patterning of silicon via ultrashort pulsed laser synthesis}

\subsubsection{Introduction}

The need to control the growth of cells is paramount in implant-body interactions as well as biosensor technologies [239]. There are a variety of techniques that have been proposed to induce surface patterning such as lithography, photolithography and micro contact printing [24], [26], [28]. However, these techniques do not provide single step selective patterning without the addition of layers and catalysts. Ultrashort pulsed laser synthesis provides a catalyst-free, single step mode of selectively patterning the surface of silicon. Ultrafast pulsed laser synthesis can be tuned to precisely and selectively generate areas of low temperature and high temperature phases of silicon and silicon oxides.

\subsubsection{Preliminary Results}

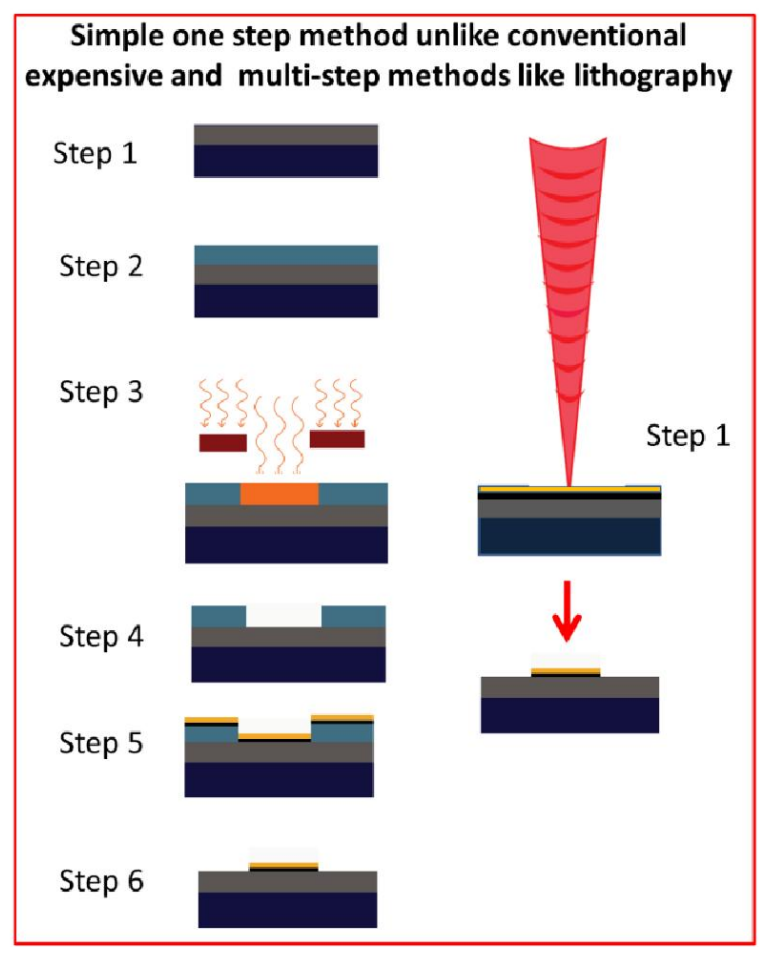




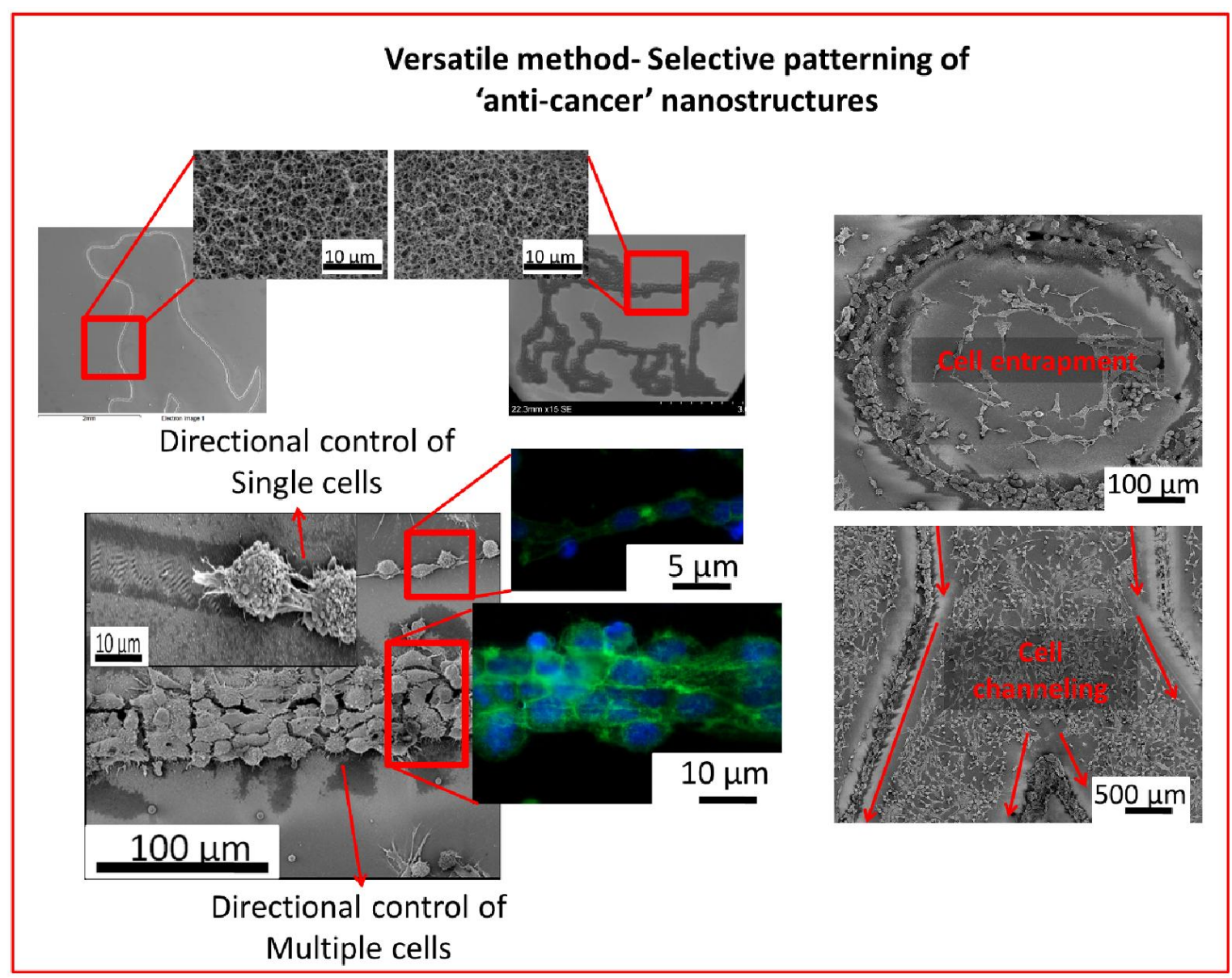

Figure 9-5: Surface patterning of 'anti-cancer' nanostructures and its applications.

\subsubsection{Proliferation, control and manipulation on other substrates}

This dissertation is the first step in generating cell proliferative or cell controlling /manipulative surfaces. A thorough study of the fabrication mechanism and characterization of the material was performed. This project has promising prospects in an array of applications such as drug delivery, tissue engineering, biosensing and lab-on-a-chop devises. Through the course of this research the properties of only silicon was studied. Investigation of different materials such as carbon, aluminum and polymers can be carried out to replicate similar results.

\subsubsection{Exploring cell-biomaterial interaction}

The cell-biomaterial interaction properties can further be probed to tailor the composition and properties of the biomaterial to suit applications. For instance, in case of more aggressive forms of cancer, increase in certain phases of nanofibrous structures can be made. 


\subsubsection{Stem cell control and proliferation}

Stem cell differentiation has shown to be greatly dependent on its environment. Therefore, applications of the three dimensional nanofibrous network can be investigated to explore differentiation into bone lineage.

\subsubsection{Bacterial control and proliferation}

With respect to the cell controlling substrates, they can applied to anti-bacterial uses. The substrate will have to be further studied and modified to obtain precise bacterial control.

\subsubsection{Natural Biomaterials}

Natural biomaterials provide a 'green' alternative to synthetic biomaterials. The synthesis via laser ablation can be studied to produce three dimensional 'green' nanofibrous substrates. 


\section{Appendix A}

\section{Published Articles}

1. Priyatha Premnath, Bo Tan, Krishnan Venkatakrishnan. Bioactive interlinked extracellular matrix-like silicon nano-network fabricated by femtosecond laser synthesis. Biores Open Access. 2012. 1(5): 231-238.

2. Priyatha Premnath, Bo Tan, Krishnan Venkatakrishnan. Direct patterning of free standing three dimensional silicon nanofibrous network to facilitate multi-dimensional growth of fibroblasts and osteoblasts. Journal of Biomedical Nanotechnology. 2013. 9 (11): 18751881. (Impact Factor: 7.578)

3. Priyatha Premnath, Bo Tan, Krishnan Venkatakrishnan. Nanostructured hybrid of immiscible gold and silicon and its effects on proliferation and adhesion of fibroblasts and osteoblasts. Journal of Biomedical Nanotechnology. 2014. 10(6): 1061-1067. (Impact Factor: 7.578)

\section{Articles under Review}

1. Priyatha Premnath, Bo Tan, Krishnan Venkatakrishnan. Programming cell fate on biofunctionalized repelling silicon. Journal of Materials Chemistry B. (Impact factor: 6.626)

\section{Submitted to refereed journals}

1. Priyatha Premnath, Bo Tan, Krishnan Venkatakrishnan. Programming cancer through phase transformed functionalized silicon based intelligent biomaterials. Chemical Science. (Impact factor: 8.601)

2. Priyatha Premnath, Bo Tan, Krishnan Venkatakrishnan. Engineering next generation functionalized multi-phase silicon/silicon oxide nano biomaterials to passivate the aggressive proliferation of cancer. Nature Communications. (Impact factor: 10.742)

3. Priyatha Premnath, Bo Tan, Krishnan Venkatakrishnan. Next generation biofunctionalized rare phased gold-silicon/silicon oxidenanohybrid biomaterials. Angewandte Chemie International Edition. (Impact Factor 13.734) 


\section{References}

[1] J. Ramsden, Biomedical surfaces. Artech House, 2008.

[2] K. S. Rho, L. Jeong, G. Lee, B.-M. Seo, Y. J. Park, S.-D. Hong, S. Roh, J. J. Cho, W. H. Park, and B.-M. Min, "Electrospinning of collagen nanofibers: effects on the behavior of normal human keratinocytes and early-stage wound healing," Biomaterials, vol. 27, no. 8, pp. 1452-1461, 2006.

[3] X. M. Mo, C. Y. Xu, M. Kotaki, and S. Ramakrishna, "Electrospun P (LLA-CL) nanofiber: a biomimetic extracellular matrix for smooth muscle cell and endothelial cell proliferation," Biomaterials, vol. 25, no. 10, pp. 1883-1890, 2004.

[4] D. D. Deligianni, N. D. Katsala, P. G. Koutsoukos, and Y. F. Missirlis, "Effect of surface roughness of hydroxyapatite on human bone marrow cell adhesion, proliferation, differentiation and detachment strength,” Biomaterials, vol. 22, no. 1, pp. 87-96, 2000.

[5] L. P. Zanello, B. Zhao, H. Hu, and R. C. Haddon, "Bone cell proliferation on carbon nanotubes," Nano Lett., vol. 6, no. 3, pp. 562-567, 2006.

[6] M. J. Dalby, D. Pasqui, and S. Affrossman, "Cell response to nano-islands produced by polymer demixing: a brief review," in IEE Proceedings-Nanobiotechnology, 2004, vol. 151, pp. 53-61.

[7] K. C. Popat, E. E. Leary Swan, V. Mukhatyar, K.-I. Chatvanichkul, G. K. Mor, C. A. Grimes, and T. A. Desai, "Influence of nanoporous alumina membranes on long-term osteoblast response," Biomaterials, vol. 26, no. 22, pp. 4516-4522, 2005.

[8] B. B. Mandal and S. C. Kundu, "Cell proliferation and migration in silk fibroin 3D scaffolds," Biomaterials, vol. 30, no. 15, pp. 2956-2965, 2009.

[9] S. L. Ishaug, G. M. Crane, M. J. Miller, A. W. Yasko, M. J. Yaszemski, and A. G. Mikos, "Bone formation by three-dimensional stromal osteoblast culture in biodegradable polymer scaffolds," J. Biomed. Mater. Res., vol. 36, no. 1, pp. 17-28, 1998.

[10] K. Cai, J. Bossert, and K. D. Jandt, "Does the nanometre scale topography of titanium influence protein adsorption and cell proliferation?," Colloids Surf. B Biointerfaces, vol. 49, no. 2, pp. 136-144, 2006. 
[11] D. Li and Y. Xia, "Electrospinning of nanofibers: reinventing the wheel?," Adv. Mater., vol. 16, no. 14, pp. 1151-1170, 2004.

[12] L. Lao, Y. Wang, Y. Zhu, Y. Zhang, and C. Gao, "Poly (lactide-coglycolide)/hydroxyapatite nanofibrous scaffolds fabricated by electrospinning for bone tissue engineering," J. Mater. Sci. Mater. Med., vol. 22, no. 8, pp. 1873-1884, 2011.

[13] A. Nandakumar, Z. T. Birgani, D. Santos, A. Mentink, N. Auffermann, K. van der Werf, M. Bennink, L. Moroni, C. van Blitterswijk, and P. Habibovic, "Surface modification of electrospun fibre meshes by oxygen plasma for bone regeneration," Biofabrication, vol. 5, no. 1, p. 015006, 2013.

[14] M. J. Dalby, M. O. Riehle, H. Johnstone, S. Affrossman, and A. S. G. Curtis, "In vitro reaction of endothelial cells to polymer demixed nanotopography," Biomaterials, vol. 23, no. 14, pp. 2945-2954, 2002.

[15] J. Y. Lim, A. D. Dreiss, Z. Zhou, J. C. Hansen, C. A. Siedlecki, R. W. Hengstebeck, J. Cheng, N. Winograd, and H. J. Donahue, "The regulation of integrin-mediated osteoblast focal adhesion and focal adhesion kinase expression by nanoscale topography," Biomaterials, vol. 28, no. 10, pp. 1787-1797, 2007.

[16] E. T. Den Braber, J. E. De Ruijter, H. T. J. Smits, L. A. Ginsel, A. F. Von Recum, and J. A. Jansen, "Quantitative analysis of cell proliferation and orientation on substrata with uniform parallel surface micro-grooves," Biomaterials, vol. 17, no. 11, pp. 1093-1099, 1996.

[17] R. S. Tu and M. Tirrell, "Bottom-up design of biomimetic assemblies," Adv. Drug Deliv. Rev., vol. 56, no. 11, pp. 1537-1563, 2004.

[18] K. Cai, M. Frant, J. Bossert, G. Hildebrand, K. Liefeith, and K. D. Jandt, "Surface functionalized titanium thin films: zeta-potential, protein adsorption and cell proliferation," Colloids Surf. B Biointerfaces, vol. 50, no. 1, pp. 1-8, 2006.

[19] X. Xing, H. Yu, D. Zhu, J. Zheng, H. Chen, W. Chen, and J. Cai, "Subwavelength and Nanometer Diameter Optical Polymer Fibers as Building Blocks for Miniaturized Photonics Integration," 2012.

[20] H. D. Kim, E. H. Bae, I. C. Kwon, R. R. Pal, J. D. Nam, and D. S. Lee, "Effect of PEGPLLA diblock copolymer on macroporous PLLA scaffolds by thermally induced phase separation," Biomaterials, vol. 25, no. 12, pp. 2319-2329, 2004. 
[21] S. A. Ruiz and C. S. Chen, "Microcontact printing: a tool to pattern," Soft Matter, vol. 3, no. 2, pp. 168-177, 2007.

[22] N. M. Rodriguez, R. A. Desai, B. Trappmann, B. M. Baker, and C. S. Chen, "Micropatterned Multicolor Dynamically Adhesive Substrates to Control Cell Adhesion and Multicellular Organization," Langmuir, vol. 30, no. 5, pp. 1327-1335, 2014.

[23] H. Aubin, J. W. Nichol, C. B. Hutson, H. Bae, A. L. Sieminski, D. M. Cropek, P. Akhyari, and A. Khademhosseini, "Directed 3D cell alignment and elongation in microengineered hydrogels," Biomaterials, vol. 31, no. 27, pp. 6941-6951, 2010.

[24] A. Revzin, R. G. Tompkins, and M. Toner, "Surface engineering with poly (ethylene glycol) photolithography to create high-density cell arrays on glass," Langmuir, vol. 19, no. 23, pp. 9855-9862, 2003.

[25] W.-G. Koh, A. Revzin, A. Simonian, T. Reeves, and M. Pishko, "Control of mammalian cell and bacteria adhesion on substrates micropatterned with poly (ethylene glycol) hydrogels," Biomed. Microdevices, vol. 5, no. 1, pp. 11-19, 2003.

[26] V. N. Truskett and M. P. Watts, "Trends in imprint lithography for biological applications," TRENDS Biotechnol., vol. 24, no. 7, pp. 312-317, 2006.

[27] K. E. Schmalenberg and K. E. Uhrich, "Micropatterned polymer substrates control alignment of proliferating Schwann cells to direct neuronal regeneration," Biomaterials, vol. 26, no. 12, pp. 1423-1430, 2005.

[28] I. Elloumi Hannachi, K. Itoga, Y. Kumashiro, J. Kobayashi, M. Yamato, and T. Okano, "Fabrication of transferable micropatterned-co-cultured cell sheets with microcontact printing," Biomaterials, vol. 30, no. 29, pp. 5427-5432, 2009.

[29] H. Zeng and W. R. Lacefield, "XPS, EDX and FTIR analysis of pulsed laser deposited calcium phosphate bioceramic coatings: the effects of various process parameters," Biomaterials, vol. 21, no. 1, pp. 23-30, 2000.

[30] L. T. de Jonge, S. C. Leeuwenburgh, J. G. Wolke, and J. A. Jansen, "Organic-inorganic surface modifications for titanium implant surfaces," Pharm. Res., vol. 25, no. 10, pp. 2357-2369, 2008.

[31] A. Ranella, M. Barberoglou, S. Bakogianni, C. Fotakis, and E. Stratakis, "Tuning cell adhesion by controlling the roughness and wettability of 3D micro/nano silicon structures," Acta Biomater., vol. 6, no. 7, pp. 2711-2720, 2010. 
[32] N. Koufaki, A. Ranella, K. E. Aifantis, M. Barberoglou, S. Psycharakis, C. Fotakis, and E. Stratakis, "Controlling cell adhesion via replication of laser micro/nano-textured surfaces on polymers," Biofabrication, vol. 3, no. 4, p. 045004, 2011.

[33] E. Rebollar, I. Frischauf, M. Olbrich, T. Peterbauer, S. Hering, J. Preiner, P. Hinterdorfer, C. Romanin, and J. Heitz, "Proliferation of aligned mammalian cells on lasernanostructured polystyrene," Biomaterials, vol. 29, no. 12, pp. 1796-1806, 2008.

[34] W. Y. Yeong, H. Yu, K. P. Lim, K. L. G. Ng, Y. C. F. Boey, V. S. Subbu, and L. P. Tan, "Multiscale topological guidance for cell alignment via direct laser writing on biodegradable polymer," Tissue Eng. Part C Methods, vol. 16, no. 5, pp. 1011-1021, 2010.

[35] R. Narayan and P. Goering, "Laser micro-and nanofabrication of biomaterials," $M R S$ Bull., vol. 36, no. 12, pp. 973-982, 2011.

[36] S. Manickam, K. Venkatakrishnan, B. Tan, and V. Venkataramanan, "Study of silicon nanofibrous structure formed by femtosecond laser irradiation in air," Opt. Express, vol. 17, no. 16, pp. 13869-13874, 2009.

[37] K. Venkatakrishnan, D. Vipparty, and B. Tan, "Nanofibre fabrication by femtosecond laser ablation of silica glass," Opt. Express, vol. 19, no. 17, pp. 15770-15776, 2011.

[38] A. Tavangar, B. Tan, and K. Venkatakrishnan, "Synthesis of three-dimensional calcium carbonate nanofibrous structure from eggshell using femtosecond laser ablation," $J$. Nanobiotechnology, vol. 9, pp. 1-1, 2011.

[39] M. Sivakumar, K. Venkatakrishnan, and B. Tan, "Study of metallic fibrous nanoparticle aggregate produced using femtosecond laser radiation under ambient conditions," Nanotechnology, vol. 21, no. 22, p. 225601, 2010.

[40] A. Tavangar, B. Tan, and K. Venkatakrishnan, "Synthesis of bio-functionalized threedimensional titania nanofibrous structures using femtosecond laser ablation," Acta Biomater., vol. 7, no. 6, pp. 2726-2732, 2011.

[41] A. Tavangar, B. Tan, and K. Venkatakrishnan, "The Influence of Laser-Induced 3-D Titania Nanofibrous Platforms on Cell Behavior," J. Biomed. Nanotechnol., vol. 9, no. 11, pp. 1837-1846, 2013.

[42] S. F. Badylak, "The extracellular matrix as a biologic scaffold material," Biomaterials, vol. 28, no. 25, pp. 3587-3593, 2007. 
[43] L. A. Smith and P. X. Ma, "Nano-fibrous scaffolds for tissue engineering," Colloids Surf. B Biointerfaces, vol. 39, no. 3, pp. 125-131, 2004.

[44] J.-P. St-Pierre, M. Gauthier, L.-P. Lefebvre, and M. Tabrizian, "Three-dimensional growth of differentiating MC3T3-E1 pre-osteoblasts on porous titanium scaffolds," Biomaterials, vol. 26, no. 35, pp. 7319-7328, 2005.

[45] H. Suh, J.-C. Park, D.-W. Han, D. H. Lee, and C. D. Han, “A bone replaceable artificial bone substitute: cytotoxicity, cell adhesion, proliferation, and alkaline phosphatase activity," Artif. Organs, vol. 25, no. 1, pp. 14-21, 2001.

[46] M. P. Staiger, A. M. Pietak, J. Huadmai, and G. Dias, "Magnesium and its alloys as orthopedic biomaterials: a review," Biomaterials, vol. 27, no. 9, pp. 1728-1734, 2006.

[47] P. Ducheyne and Q. Qiu, "Bioactive ceramics: the effect of surface reactivity on bone formation and bone cell function," Biomaterials, vol. 20, no. 23, pp. 2287-2303, 1999.

[48] J. L. Coffer, M. A. Whitehead, D. K. Nagesha, P. Mukherjee, G. Akkaraju, M. Totolici, R. S. Saffie, and L. T. Canham, "Porous silicon-based scaffolds for tissue engineering and other biomedical applications," Phys. Status Solidi A, vol. 202, no. 8, pp. 1451-1455, 2005.

[49] C. Vitale-Brovarone, E. Verné, L. Robiglio, P. Appendino, F. Bassi, G. Martinasso, G. Muzio, and R. Canuto, "Development of glass-ceramic scaffolds for bone tissue engineering: characterisation, proliferation of human osteoblasts and nodule formation," Acta Biomater., vol. 3, no. 2, pp. 199-208, 2007.

[50] L. T. Canham, "Bioactive silicon structure fabrication through nanoetching techniques," Adv. Mater., vol. 7, no. 12, pp. 1033-1037, 1995.

[51] W. Sun, J. E. Puzas, T.-J. Sheu, and P. M. Fauchet, "Porous silicon as a cell interface for bone tissue engineering," Phys. Status Solidi A, vol. 204, no. 5, pp. 1429-1433, 2007.

[52] A. A. Agrawal, B. J. Nehilla, K. V. Reisig, T. R. Gaborski, D. Z. Fang, C. C. Striemer, P. M. Fauchet, and J. L. McGrath, "Porous nanocrystalline silicon membranes as highly permeable and molecularly thin substrates for cell culture," Biomaterials, vol. 31, no. 20, pp. 5408-5417, 2010.

[53] J. Lee, B. H. Chu, K.-H. Chen, F. Ren, and T. P. Lele, "Randomly oriented, upright $\mathrm{SiO}<$ sub $>2</$ sub $>$ coated nanorods for reduced adhesion of mammalian cells," Biomaterials, vol. 30 , no. 27 , pp. 4488-4493, 2009. 
[54] E. S. Thian, J. Huang, S. M. Best, Z. H. Barber, R. A. Brooks, N. Rushton, and W. Bonfield, "The response of osteoblasts to nanocrystalline silicon-substituted hydroxyapatite thin films," Biomaterials, vol. 27, no. 13, pp. 2692-2698, 2006.

[55] W. Kim, J. K. Ng, M. E. Kunitake, B. R. Conklin, and P. Yang, "Interfacing silicon nanowires with mammalian cells," J. Am. Chem. Soc., vol. 129, no. 23, pp. 7228-7229, 2007.

[56] R. Nazarov, H.-J. Jin, and D. L. Kaplan, "Porous 3-D scaffolds from regenerated silk fibroin," Biomacromolecules, vol. 5, no. 3, pp. 718-726, 2004.

[57] B. L. Behan, D. G. DeWitt, D. R. Bogdanowicz, A. N. Koppes, S. S. Bale, and D. M. Thompson, "Single-walled carbon nanotubes alter Schwann cell behavior differentially within 2D and 3D environments," J. Biomed. Mater. Res. A, vol. 96, no. 1, pp. 46-57, 2011.

[58] E. Cukierman, R. Pankov, and K. M. Yamada, "Cell interactions with three-dimensional matrices," Curr. Opin. Cell Biol., vol. 14, no. 5, pp. 633-640, 2002.

[59] B. Tan and K. Venkatakrishnan, "Synthesis of fibrous nanoparticle aggregates by femtosecond laser ablation in air," Opt. Express, vol. 17, no. 2, pp. 1064-1069, 2009.

[60] T. Kokubo and H. Takadama, "How useful is SBF in predicting in vivo bone bioactivity?," Biomaterials, vol. 27, no. 15, pp. 2907-2915, 2006.

[61] T. Mosmann, "Rapid colorimetric assay for cellular growth and survival: application to proliferation and cytotoxicity assays," J Immunol Methods, vol. 65, no. 1-2, pp. 55-63, 1983.

[62] M. S. Tillack, D. W. Blair, and S. S. Harilal, "The effect of ionization on cluster formation in laser ablation plumes," Nanotechnology, vol. 15, no. 3, p. 390, 2004.

[63] A. Tavangar, B. Tan, and K. Venkatakrishnan, "Single-step fabrication of microfluidic channels filled with nanofibrous membrane using femtosecond laser irradiation," $J$. Micromechanics Microengineering, vol. 20, no. 8, p. 085016, 2010.

[64] A. Kishida, H. Iwata, Y. Tamada, and Y. Ikada, "Cell behaviour on polymer surfaces grafted with non-ionic and ionic monomers," Biomaterials, vol. 12, no. 8, pp. 786-792, 1991. 
[65] G. Sonavane, K. Tomoda, and K. Makino, "Biodistribution of colloidal gold nanoparticles after intravenous administration: effect of particle size," Colloids Surf. B Biointerfaces, vol. 66, no. 2, pp. 274-280, 2008.

[66] P. B. Van Wachem, A. H. Hogt, T. Beugeling, J. Feijen, A. Bantjes, J. P. Detmers, and W. G. Van Aken, "Adhesion of cultured human endothelial cells onto methacrylate polymers with varying surface wettability and charge," Biomaterials, vol. 8, no. 5, pp. 323-328, 1987.

[67] H. H. P. Fang, K. Y. Chan, and L. C. Xu, "Quantification of bacterial adhesion forces using atomic force microscopy (AFM)," J. Microbiol. Methods, vol. 40, no. 1, pp. 89-97, 2000.

[68] S. Fujibayashi, M. Neo, H. M. Kim, T. Kokubo, and T. Nakamura, "Osteoinduction of porous bioactive titanium metal," Biomaterials, vol. 25, no. 3, pp. 443-450, 2004.

[69] A. M. Pietak, J. W. Reid, M. J. Stott, and M. Sayer, "Silicon substitution in the calcium phosphate bioceramics," Biomaterials, vol. 28, no. 28, pp. 4023-4032, 2007.

[70] W. J. Li, C. T. Laurencin, E. J. Caterson, R. S. Tuan, and F. K. Ko, "Electrospun nanofibrous structure: a novel scaffold for tissue engineering," J. Biomed. Mater. Res., vol. 60, no. 4, pp. 613-621, 2002.

[71] C. H. Choi, S. H. Hagvall, B. M. Wu, J. C. Y. Dunn, and R. E. Beygui, "Cell interaction with three-dimensional sharp-tip nanotopography," Biomaterials, vol. 28, no. 9, pp. 1672$1679,2007$.

[72] M. M. Stevens and J. H. George, "Exploring and engineering the cell surface interface," Science, vol. 310, no. 5751, pp. 1135-1138, 2005.

[73] L. G. Griffith and M. A. Swartz, "Capturing complex 3D tissue physiology in vitro," Nat. Rev. Mol. Cell Biol., vol. 7, no. 3, pp. 211-224, 2006.

[74] K. M. Yamada and E. Cukierman, "Modeling tissue morphogenesis and cancer in 3D," Cell, vol. 130, no. 4, pp. 601-610, 2007.

[75] E. Cukierman, R. Pankov, D. R. Stevens, and K. M. Yamada, "Taking cell-matrix adhesions to the third dimension," Sci. STKE, vol. 294, no. 5547, p. 1708, 2001.

[76] A. G. Mikos, A. J. Thorsen, L. A. Czerwonka, Y. Bao, R. Langer, D. N. Winslow, and J. P. Vacanti, "Preparation and characterization of poly (L-lactic acid) foams," Polymer, vol. 35, no. 5, pp. 1068-1077, 1994. 
[77] L. Lu, S. J. Peter, M. D. Lyman, H. L. Lai, S. M. Leite, J. A Tamada, J. P. Vacanti, R. Langer, and A. G. Mikos, "In vitro degradation of porous poly (-lactic acid) foams," Biomaterials, vol. 21, no. 15, pp. 1595-1605, 2000.

[78] B. Leukers, H. Gülkan, S. H. Irsen, S. Milz, C. Tille, M. Schieker, and H. Seitz, "Hydroxyapatite scaffolds for bone tissue engineering made by 3D printing," J. Mater. Sci. Mater. Med., vol. 16, no. 12, pp. 1121-1124, 2005.

[79] J. Doshi and D. H. Reneker, "Electrospinning process and applications of electrospun fibers," J. Electrost., vol. 35, no. 2-3, pp. 151-160, 1995.

[80] V. Milleret, T. Hefti, H. Hall, V. Vogel, and D. Eberli, "Influence of fiber diameter and surface roughness of electrospun vascular grafts on blood activation," Acta Biomater., 2012.

[81] R. Murugan and S. Ramakrishna, "Nano-featured scaffolds for tissue engineering: a review of spinning methodologies," Tissue Eng., vol. 12, no. 3, pp. 435-447, 2006.

[82] Q. Wang, X. Hu, Y. Du, and J. F. Kennedy, "Alginate/starch blend fibers and their properties for drug controlled release," Carbohydr. Polym., vol. 82, no. 3, pp. 842-847, 2010.

[83] Q. Wang, N. Zhang, X. Hu, J. Yang, and Y. Du, "Chitosan/polyethylene glycol blend fibers and their properties for drug controlled release," J. Biomed. Mater. Res. A, vol. 85, no. 4, pp. 881-887, 2008.

[84] S. P. Low, N. H. Voelcker, L. T. Canham, and K. A. Williams, "The biocompatibility of porous silicon in tissues of the eye," Biomaterials, vol. 30, no. 15, pp. 2873-2880, 2009.

[85] Y. W. Fan, F. Z. Cui, S. P. Hou, Q. Y. Xu, L. N. Chen, and I. S. Lee, "Culture of neural cells on silicon wafers with nano-scale surface topograph," J. Neurosci. Methods, vol. 120, no. 1, pp. 17-23, 2002.

[86] K. Jiang, J. L. Coffer, and G. R. Akkaraju, "Silicon nanowire/polycaprolactone composites and their impact on stromal cell function," J. Mater. Res., vol. 1, no. 1, pp. 18, 2012.

[87] E. M. Carlisle, "In vivo requirement for silicon in articular cartilage and connective tissue formation in the chick.," J. Nutr., vol. 106, no. 4, p. 478, 1976.

[88] E. M. Carlisle, "Biochemical and morphological changes associated with long bone abnormalities in silicon deficiency.," J. Nutr., vol. 110, no. 5, p. 1046, 1980. 
[89] A. Tavangar, B. Tan, and K. Venkatakrishnan, "Deposition of fibrous nanostructure by ultrafast laser ablation," J. Micromechanics Microengineering, vol. 20, no. 5, p. 055002, 2010.

[90] A. Tavangar, B. Tan, and K. Venkatakrishnan, "Synthesis of three-dimensional calcium carbonate nanofibrous structure from eggshell using femtosecond laser ablation," $J$. Nanobiotechnology, vol. 9, pp. 1-1, 2011.

[91] M. Sivayoganathan, B. Tan, and K. Venkatakrishnan, "Synthesis of crystalline and amorphous, particle-agglomerated 3-D nanostructures of $\mathrm{Al}$ and $\mathrm{Si}$ oxides by femtosecond laser and the prediction of these particle sizes," Nanoscale Res. Lett., vol. 7, no. 1, pp. 1-7, 2012.

[92] R. Hergenröder, "Laser-generated aerosols in laser ablation for inductively coupled plasma spectrometry," Spectrochim. Acta Part B At. Spectrosc., vol. 61, no. 3, pp. 284$300,2006$.

[93] A. Kiani, K. Venkatakrishnan, and B. Tan, "Direct patterning of silicon oxide on Sisubstrate induced by femtosecond laser," Opt. Express, vol. 18, no. 3, pp. 1872-1878, 2010.

[94] P. Premnath, B. Tan, and K. Venkatakrishnan, "Bioactive Interlinked Extracellular Matrix-Like Silicon Nano-Network Fabricated by Femtosecond Laser Synthesis," BioResearch Open Access.

[95] M. J. Dalby, S. J. Yarwood, M. O. Riehle, H. J. H. Johnstone, S. Affrossman, and A. S. G. Curtis, "Increasing fibroblast response to materials using nanotopography: morphological and genetic measurements of cell response to 13-nm-high polymer demixed islands," Exp. Cell Res., vol. 276, no. 1, pp. 1-9, 2002.

[96] M. J. Dalby, M. O. Riehle, H. J. H. Johnstone, S. Affrossman, and A. S. G. Curtis, "Polymer-demixed nanotopography: control of fibroblast spreading and proliferation," Tissue Eng., vol. 8, no. 6, pp. 1099-1108, 2002.

[97] P. Linez-Bataillon, F. Monchau, M. Bigerelle, and H. F. Hildebrand, "In vitro MC3T3 osteoblast adhesion with respect to surface roughness of Ti6Al4V substrates," Biomol. Eng., vol. 19, no. 2, pp. 133-141, 2002. 
[98] J. R. Venugopal, Y. Zhang, and S. Ramakrishna, "In vitro culture of human dermal fibroblasts on electrospun polycaprolactone collagen nanofibrous membrane," Artif. Organs, vol. 30, no. 6, pp. 440-446, 2006.

[99] Y. Z. Zhang, J. Venugopal, Z. M. Huang, C. T. Lim, and S. Ramakrishna, "Characterization of the surface biocompatibility of the electrospun PCL-collagen nanofibers using fibroblasts," Biomacromolecules, vol. 6, no. 5, pp. 2583-2589, 2005.

[100] C. T. Laurencin, A. M. A. Ambrosio, M. D. Borden, and J. A. Cooper Jr, "Tissue engineering: orthopedic applications," Annu. Rev. Biomed. Eng., vol. 1, no. 1, pp. 19-46, 1999.

[101] T. P. Kunzler, T. Drobek, M. Schuler, and N. D. Spencer, "Systematic study of osteoblast and fibroblast response to roughness by means of surface-morphology gradients," Biomaterials, vol. 28, no. 13, pp. 2175-2182, 2007.

[102] F. Haq, V. Anandan, C. Keith, and G. Zhang, "Neurite development in PC12 cells cultured on nanopillars and nanopores with sizes comparable with filopodia," Int. J. Nanomedicine, vol. 2, no. 1, p. 107, 2007.

[103] S. H. Oh, I. K. Park, J. M. Kim, and J. H. Lee, "In vitro and in vivo characteristics of PCL scaffolds with pore size gradient fabricated by a centrifugation method," Biomaterials, vol. 28, no. 9, pp. 1664-1671, 2007.

[104] J. P. St-Pierre, M. Gauthier, L. P. Lefebvre, and M. Tabrizian, "Three-dimensional growth of differentiating MC3T3-E1 pre-osteoblasts on porous titanium scaffolds," Biomaterials, vol. 26, no. 35, pp. 7319-7328, 2005.

[105] X. Liu, J. M. Holzwarth, and P. X. Ma, "Functionalized Synthetic Biodegradable Polymer Scaffolds for Tissue Engineering," Macromol. Biosci., 2012.

[106] P. Ducheyne and Q. Qiu, "Bioactive ceramics: the effect of surface reactivity on bone formation and bone cell function," Biomaterials, vol. 20, no. 23, pp. 2287-2303, 1999.

[107] C. Vitale-Brovarone, E. Verné, L. Robiglio, P. Appendino, F. Bassi, G. Martinasso, G. Muzio, and R. Canuto, "Development of glass-ceramic scaffolds for bone tissue engineering: characterisation, proliferation of human osteoblasts and nodule formation," Acta Biomater., vol. 3, no. 2, pp. 199-208, 2007. 
[108] K. Chen, S. Sahoo, P. He, K. S. Ng, S. L. Toh, and J. C. H. Goh, "A Hybrid Silk/RADABased Fibrous Scaffold with Triple Hierarchy for Ligament Regeneration," Tissue Eng. Part A, vol. 18, no. 13-14, pp. 1399-1409, 2012.

[109] B. Lei, K. H. Shin, D. Y. Noh, I. H. Jo, Y. H. Koh, W. Y. Choi, and H. E. Kim, "Nanofibrous gelatin/silica hybrid scaffolds mimicking native extracellular matrix (ECM) using thermally induced phase separation," J. Mater. Chem., 2012.

[110] Y. Pan, S. Neuss, A. Leifert, M. Fischler, F. Wen, U. Simon, G. Schmid, W. Brandau, and W. Jahnen-Dechent, "Size-Dependent Cytotoxicity of Gold Nanoparticles," Small, vol. 3, no. 11, pp. 1941-1949, 2007.

[111] R. Shukla, V. Bansal, M. Chaudhary, A. Basu, R. R. Bhonde, and M. Sastry, "Biocompatibility of gold nanoparticles and their endocytotic fate inside the cellular compartment: a microscopic overview," Langmuir, vol. 21, no. 23, pp. 10644-10654, 2005.

[112] E. E. Connor, J. Mwamuka, A. Gole, C. J. Murphy, and M. D. Wyatt, "Gold nanoparticles are taken up by human cells but do not cause acute cytotoxicity," Small, vol. 1, no. 3, pp. 325-327, 2005.

[113] G. M. Kim, A. Wutzler, H. J. Radusch, G. H. Michler, P. Simon, R. A. Sperling, and W. J. Parak, "One-dimensional arrangement of gold nanoparticles by electrospinning," Chem. Mater., vol. 17, no. 20, pp. 4949-4957, 2005.

[114] J. Lee, B. H. Chu, K. H. Chen, F. Ren, and T. P. Lele, "Randomly oriented, upright $\mathrm{SiO}<$ sub $>2</$ sub $>$ coated nanorods for reduced adhesion of mammalian cells," Biomaterials, vol. 30, no. 27, pp. 4488-4493, 2009.

[115] B. Chevallay, N. Abdul-Malak, and D. Herbage, "Mouse fibroblasts in long-term culture within collagen three-dimensional scaffolds: Influence of crosslinking with diphenylphosphorylazide on matrix reorganization, growth, and biosynthetic and proteolytic activities," J. Biomed. Mater. Res., vol. 49, no. 4, pp. 448-459, 2000.

[116] M. Sivayoganathan, B. Tan, and K. Venkatakrishnan, "Effect of mega-hertz repetition rate on the agglomerated particle size of femtosecond synthesized nanostructures," Opt. Mater. Express, vol. 2, no. 8, pp. 987-995, 2012. 
[117] J. F. Chang, T. F. Young, Y. L. Yang, H. Y. Ueng, and T. C. Chang, "Silicide formation of Au thin films on (100) Si during annealing," Mater. Chem. Phys., vol. 83, no. 2, pp. 199-203, 2004.

[118] F. Kozin, B. Millstein, G. Mandel, and N. Mandel, "Silica-induced membranolysis: a study of different structural forms of crystalline and amorphous silica and the effects of protein adsorption," J. Colloid Interface Sci., vol. 88, no. 2, pp. 326-337, 1982.

[119] V. Lindroos, M. Tilli, A. Lehto, and T. Motooka, Handbook of silicon based MEMS materials and technologies. William Andrew, 2009.

[120] J. L. Coffer, M. A. Whitehead, D. K. Nagesha, P. Mukherjee, G. Akkaraju, M. Totolici, R. S. Saffie, and L. T. Canham, "Porous silicon-based scaffolds for tissue engineering and other biomedical applications," Phys. Status Solidi A, vol. 202, no. 8, pp. 1451-1455, 2005.

[121] H. M. Ji, V. Samper, Y. Chen, C. K. Heng, T. M. Lim, and L. Yobas, "Silicon-based microfilters for whole blood cell separation," Biomed. Microdevices, vol. 10, no. 2, pp. 251-257, 2008.

[122] B. Godin, C. Chiappini, S. Srinivasan, J. F. Alexander, K. Yokoi, M. Ferrari, P. Decuzzi, and X. Liu, "Discoidal porous silicon particles: fabrication and biodistribution in breast cancer bearing mice," Adv. Funct. Mater., vol. 22, no. 20, pp. 4225-4235, 2012.

[123] H. G. Craighead, C. D. James, and A. M. P. Turner, "Chemical and topographical patterning for directed cell attachment," Curr. Opin. Solid State Mater. Sci., vol. 5, no. 2, pp. 177-184, 2001.

[124] M. Ni, W. H. Tong, D. Choudhury, N. A. A. Rahim, C. Iliescu, and H. Yu, "Cell culture on MEMS platforms: A review," Int. J. Mol. Sci., vol. 10, no. 12, pp. 5411-5441, 2009.

[125] A. Curtis and C. Wilkinson, "Topographical control of cells," Biomaterials, vol. 18, no. 24, pp. 1573-1583, 1997.

[126] S. Sharma, R. W. Johnson, and T. A. Desai, "Evaluation of the stability of nonfouling ultrathin poly (ethylene glycol) films for silicon-based microdevices," Langmuir, vol. 20, no. 2, pp. 348-356, 2004.

[127] H.-A. Pan, Y.-C. Hung, Y.-P. Sui, and G. S. Huang, "Topographic control of the growth and function of cardiomyoblast H9c2 cells using nanodot arrays," Biomaterials, vol. 33, no. 1, pp. 20-28, 2012. 
[128] J. B. Recknor, D. S. Sakaguchi, and S. K. Mallapragada, "Directed growth and selective differentiation of neural progenitor cells on micropatterned polymer substrates," Biomaterials, vol. 27, no. 22, pp. 4098-4108, 2006.

[129] X. Tan, H.-J. Yoon, J. Granneman, H.-P. Moore, and M.-C. Cheng, "A high-throughput microfluidic chip for size sorting of cells," in Micro Electro Mechanical Systems (MEMS), 2011 IEEE 24th International Conference on, 2011, pp. 1075-1078.

[130] J. Y. Park, M. Morgan, A. N. Sachs, J. Samorezov, R. Teller, Y. Shen, K. J. Pienta, and S. Takayama, "Single cell trapping in larger microwells capable of supporting cell spreading and proliferation," Microfluid. Nanofluidics, vol. 8, no. 2, pp. 263-268, 2010.

[131] F. Notta, S. Doulatov, E. Laurenti, A. Poeppl, I. Jurisica, and J. E. Dick, "Isolation of single human hematopoietic stem cells capable of long-term multilineage engraftment," Science, vol. 333, no. 6039, pp. 218-221, 2011.

[132] A. Kiani, K. Venkatakrishnan, and B. Tan, "Direct laser writing of amorphous silicon on Si-substrate induced by high repetition femtosecond pulses," J. Appl. Phys., vol. 108, no. 7, pp. 074907-074907, 2010.

[133] P. R. Woodard and J. Dryden, "Thermal analysis of a laser pulse for discrete spot surface transformation hardening," J. Appl. Phys., vol. 85, no. 5, pp. 2488-2496, 1999.

[134] M. J. Smith, M.-J. Sher, B. Franta, Y.-T. Lin, E. Mazur, and S. Gradecak, "The origins of pressure-induced phase transformations during the surface texturing of silicon using femtosecond laser irradiation," J. Appl. Phys., vol. 112, no. 8, pp. 083518-083518, 2012.

[135] K. Boussu, B. Van der Bruggen, A. Volodin, J. Snauwaert, C. Van Haesendonck, and C. Vandecasteele, "Roughness and hydrophobicity studies of nanofiltration membranes using different modes of AFM," J. Colloid Interface Sci., vol. 286, no. 2, pp. 632-638, 2005.

[136] B. Fubini, V. Bolis, A. Cavenago, and M. Volante, "Physicochemical properties of crystalline silica dusts and their possible implication in various biological responses," Scand. J. Work. Environ. Health, pp. 9-14, 1995.

[137] ENS-Lyon France, "Handbook of Raman Spectra," www.ens-lyon.fr. [Online]. Available: http://www.ens-lyon.fr/LST/Raman/spectrum.php?nom=tridymite. [Accessed: 23-Jul2013].

[138] C. R. Helms and E. H. Poindexter, "The silicon-silicon dioxide system: Its microstructure and imperfections," Rep. Prog. Phys., vol. 57, no. 8, p. 791, 1994. 
[139] F. J. Grunthaner and P. J. Grunthaner, "Chemical and electronic structure of the $\mathrm{SiO}<$ sub $>2</$ sub $>/$ Si interface," Mater. Sci. Rep., vol. 1, no. 2, pp. 65-160, 1986.

[140] L. Bacakova, E. Filova, M. Parizek, T. Ruml, and V. Svorcik, "Modulation of cell adhesion, proliferation and differentiation on materials designed for body implants," Biotechnol. Adv., vol. 29, no. 6, pp. 739-767, 2011.

[141] A. Kromka, L. Grausova, L. Bacakova, J. Vacik, B. Rezek, M. Vanecek, O. A. Williams, and K. Haenen, "Semiconducting to metallic-like boron doping of nanocrystalline diamond films and its effect on osteoblastic cells," Diam. Relat. Mater., vol. 19, no. 2, pp. 190-195, 2010.

[142] H. A. Schafft, J. S. Suehle, and P. G. A. Mirel, "Thermal conductivity measurements of thin-film silicon dioxide," in Microelectronic Test Structures, 1989. ICMTS 1989. Proceedings of the 1989 International Conference on, 1989, pp. 121-125.

[143] L. Prodanov, J. te Riet, E. Lamers, M. Domanski, R. Luttge, J. J. van Loon, J. A. Jansen, and X. F. Walboomers, "The interaction between nanoscale surface features and mechanical loading and its effect on osteoblast-like cells behavior," Biomaterials, vol. 31, no. 30, pp. 7758-7765, 2010.

[144] T. Tzvetkova-Chevolleau, A. Stéphanou, D. Fuard, J. Ohayon, P. Schiavone, and P. Tracqui, "The motility of normal and cancer cells in response to the combined influence of the substrate rigidity and anisotropic microstructure," Biomaterials, vol. 29, no. 10, pp. $1541-1551,2008$.

[145] D. E. Ingber, "Tensegrity I. Cell structure and hierarchical systems biology," J. Cell Sci., vol. 116, no. 7, pp. 1157-1173, 2003.

[146] S. Lenhert, M.-B. Meier, U. Meyer, L. Chi, and H. P. Wiesmann, "Osteoblast alignment, elongation and migration on grooved polystyrene surfaces patterned by LangmuirBlodgett lithography," Biomaterials, vol. 26, no. 5, pp. 563-570, 2005.

[147] D. Peer, J. M. Karp, S. Hong, O. C. Farokhzad, R. Margalit, and R. Langer, "Nanocarriers as an emerging platform for cancer therapy," Nat. Nanotechnol., vol. 2, no. 12, pp. 751760, 2007.

[148] M. E. Dudley and S. A. Rosenberg, "Adoptive-cell-transfer therapy for the treatment of patients with cancer," Nat. Rev. Cancer, vol. 3, no. 9, pp. 666-675, 2003. 
[149] R. I. CTSU, "Effects of chemotherapy and hormonal therapy for early breast cancer on recurrence and 15-year survival: an overview of the randomised trials," Lancet, vol. 365, pp. 1687-1717, 2005.

[150] D. E. Gerber, "Targeted therapies: a new generation of cancer treatments.," Am. Fam. Physician, vol. 77, no. 3, 2008.

[151] S. B. Brown, E. A. Brown, and I. Walker, "The present and future role of photodynamic therapy in cancer treatment," Lancet Oncol., vol. 5, no. 8, pp. 497-508, 2004.

[152] Y. Xu, J. A. Phillips, J. Yan, Q. Li, Z. H. Fan, and W. Tan, "Aptamer-based microfluidic device for enrichment, sorting, and detection of multiple cancer cells," Anal. Chem., vol. 81, no. 17, pp. 7436-7442, 2009.

[153] A.-E. Saliba, L. Saias, E. Psychari, N. Minc, D. Simon, F.-C. Bidard, C. Mathiot, J.-Y. Pierga, V. Fraisier, and J. Salamero, "Microfluidic sorting and multimodal typing of cancer cells in self-assembled magnetic arrays," Proc. Natl. Acad. Sci., vol. 107, no. 33, pp. 14524-14529, 2010.

[154] S. Purushotham and R. V. Ramanujan, "Thermoresponsive magnetic composite nanomaterials for multimodal cancer therapy," Acta Biomater., vol. 6, no. 2, pp. 502-510, 2010.

[155] N. Singh, G. J. Jenkins, R. Asadi, and S. H. Doak, "Potential toxicity of superparamagnetic iron oxide nanoparticles (SPION).," Nano Rev., vol. 1, 2010.

[156] N. Lee and T. Hyeon, "Designed synthesis of uniformly sized iron oxide nanoparticles for efficient magnetic resonance imaging contrast agents," Chem. Soc. Rev., vol. 41, no. 7, pp. 2575-2589, 2012.

[157] K. H. Bae, K. Lee, C. Kim, and T. G. Park, "Surface functionalized hollow manganese oxide nanoparticles for cancer targeted siRNA delivery and magnetic resonance imaging," Biomaterials, vol. 32, no. 1, pp. 176-184, 2011.

[158] R. E. Serda, I. M. Meraz, J. Gu, X. Xia, H. Shen, T. Sun, and M. Ferrari, Mesoporous silicon particles for the presentation of tumor antigens and adjuvant for anti-cancer immunity. Google Patents, 2012.

[159] F. Peng, Y. Su, X. Wei, Y. Lu, Y. Zhou, Y. Zhong, S.-T. Lee, and Y. He, "SiliconNanowire-Based Nanocarriers with Ultrahigh Drug-Loading Capacity for In Vitro and In Vivo Cancer Therapy," Angew. Chem. Int. Ed., vol. 52, no. 5, pp. 1457-1461, 2013. 
[160] Y. Su, X. Wei, F. Peng, Y. Zhong, Y. Lu, S. Su, T. Xu, S.-T. Lee, and Y. He, “Gold nanoparticles-decorated silicon nanowires as highly efficient near-infrared hyperthermia agents for cancer cells destruction," Nano Lett., vol. 12, no. 4, pp. 1845-1850, 2012.

[161] L. Xiao, L. Gu, S. B. Howell, and M. J. Sailor, "Porous silicon nanoparticle photosensitizers for singlet oxygen and their phototoxicity against cancer cells," $A C S$ Nano, vol. 5, no. 5, pp. 3651-3659, 2011.

[162] J. Chen, J. Li, and Y. Sun, "Microfluidic approaches for cancer cell detection, characterization, and separation," Lab. Chip, vol. 12, no. 10, pp. 1753-1767, 2012.

[163] D. Von der Linde, K. Sokolowski-Tinten, and J. Bialkowski, "Laser-solid interaction in the femtosecond time regime," Appl. Surf. Sci., vol. 109, pp. 1-10, 1997.

[164] J. R. Goldman and J. A. Prybyla, "Ultrafast dynamics of laser-excited electron distributions in silicon," Phys. Rev. Lett., vol. 72, no. 9, p. 1364, 1994.

[165] J. Bonse, K.-W. Brzezinka, and A. J. Meixner, "Modifying single-crystalline silicon by femtosecond laser pulses: an analysis by micro Raman spectroscopy, scanning laser microscopy and atomic force microscopy," Appl. Surf. Sci., vol. 221, no. 1, pp. 215-230, 2004.

[166] B. N. Chichkov, C. Momma, S. Nolte, F. Von Alvensleben, and A. Tünnermann, "Femtosecond, picosecond and nanosecond laser ablation of solids," Appl. Phys. A, vol. 63, no. 2, pp. 109-115, 1996.

[167] R. J. Hemley, H.-K. Mao, and E. C. T. Chao, "Raman spectrum of natural and synthetic stishovite," Phys. Chem. Miner., vol. 13, no. 5, pp. 285-290, 1986.

[168] B. P. Glass and M. Fries, "Micro-Raman spectroscopic study of fine-grained, shockmetamorphosed rock fragments from the Australasian microtektite layer," Meteorit. Planet. Sci., vol. 43, no. 9, pp. 1487-1496, 2008.

[169] T. Hirose, K. Kihara, M. Okuno, S. Fujinami, and K. Shinoda, "X-ray, DTA and Raman studies of monoclinic tridymite and its higher temperature orthorhombic modification with varying temperature," J. Mineral. Petrol. Sci., vol. 100, no. 2, pp. 55-69, 2005.

[170] B. K. Nayak and M. C. Gupta, "Femtosecond-laser-induced-crystallization and simultaneous formation of light trapping microstructures in thin a-Si: $\mathrm{H}$ films," Appl. Phys. A, vol. 89, no. 3, pp. 663-666, 2007. 
[171] M. T. Soh, J. H. Thomas, and J. J. Talghader, "Thermally induced structural changes in nanoporous silicon dioxide from x-ray photoelectron spectroscopy," J. Vac. Sci. Technol. Vac. Surf. Films, vol. 24, no. 6, pp. 2147-2150, 2006.

[172] M. Théry, V. Racine, A. Pépin, M. Piel, Y. Chen, J.-B. Sibarita, and M. Bornens, “The extracellular matrix guides the orientation of the cell division axis," Nat. Cell Biol., vol. 7, no. 10, pp. 947-953, 2005.

[173] S. B. Carter, "Haptotaxis and the mechanism of cell motility," Nature, vol. 213, pp. 256260, 1967.

[174] S. Tojkander, G. Gateva, and P. Lappalainen, "Actin stress fibers-assembly, dynamics and biological roles," J. Cell Sci., vol. 125, no. 8, pp. 1855-1864, 2012.

[175] M. J. Dalby, M. O. Riehle, S. J. Yarwood, C. D. Wilkinson, and A. S. Curtis, "Nucleus alignment and cell signaling in fibroblasts: response to a micro-grooved topography," Exp. Cell Res., vol. 284, no. 2, pp. 272-280, 2003.

[176] Q. Y. Tang, W. Y. Tong, J. Shi, P. Shi, Y. W. Lam, and S. W. Pang, "Influence of engineered surface on cell directionality and motility," Biofabrication, vol. 6, no. 1, 2014.

[177] Y. Zhong, S. He, C. Dong, B. Ji, and G. Hu, "Cell polarization energy and its implications for cell migration," 2014.

[178] R. B. Dickinson and R. T. Tranquillo, "A stochastic model for adhesion-mediated cell random motility and haptotaxis," J. Math. Biol., vol. 31, no. 6, pp. 563-600, 1993.

[179] G. P. Raeber, M. P. Lutolf, and J. A. Hubbell, "Part II: Fibroblasts preferentially migrate in the direction of principal strain," Biomech. Model. Mechanobiol., vol. 7, no. 3, pp. 215$225,2008$.

[180] J. M. Schakenraad, H. J. Busscher, C. R. Wildevuur, and J. Arends, "Thermodynamic aspects of cell spreading on solid substrata," Cell Biophys., vol. 13, no. 1, pp. 75-91, 1988.

[181] M. C. Halloran and M. A. Wolman, "Repulsion or adhesion: receptors make the call," Curr. Opin. Cell Biol., vol. 18, no. 5, pp. 533-540, 2006.

[182] A. S. Monem, N. Elbialy, and N. Mohamed, "Mesoporous silica coated gold nanorods loaded doxorubicin for combined chemo-photothermal therapy," Int. J. Pharm., vol. 470, no. 1-2, pp. 1-7, 2014. 
[183] S. Srinivasan, R. Manchanda, T. Lei, A. Nagesetti, A. Fernandez-Fernandez, and A. J. McGoron, "Targeted nanoparticles for simultaneous delivery of chemotherapeutic and hyperthermia agents - An in vitro study," J. Photochem. Photobiol. B, vol. 136, pp. 81-90, 2014.

[184] L. Shi, Z. Chu, Y. Liu, J. Peng, and W. Jin, “Three-dimensional porous microarray of gold modified electrode for ultrasensitive and simultaneous assay of various cancer biomarkers," J. Mater. Chem. B, vol. 2, no. 18, pp. 2658-2665, 2014.

[185] L. Li, J. Xu, X. Zheng, C. Ma, X. Song, S. Ge, J. Yu, and M. Yan, "Growth of goldmanganese oxide nanostructures on a 3D origami device for glucose-oxidase label based electrochemical immunosensor,” Biosens. Bioelectron., vol. 61, pp. 76-82, 2014.

[186] F. Erogbogbo, K.-T. Yong, I. Roy, R. Hu, W.-C. Law, W. Zhao, H. Ding, F. Wu, R. Kumar, M. T. Swihart, and others, "In vivo targeted cancer imaging, sentinel lymph node mapping and multi-channel imaging with biocompatible silicon nanocrystals," ACS Nano, vol. 5, no. 1, pp. 413-423, 2010.

[187] F. Erogbogbo, K.-T. Yong, I. Roy, G. Xu, P. N. Prasad, and M. T. Swihart, "Biocompatible luminescent silicon quantum dots for imaging of cancer cells," Acs Nano, vol. 2, no. 5, pp. 873-878, 2008.

[188] I. I. Slowing, J. L. Vivero-Escoto, C.-W. Wu, and V. S.-Y. Lin, "Mesoporous silica nanoparticles as controlled release drug delivery and gene transfection carriers," $A d v$. Drug Deliv. Rev., vol. 60, no. 11, pp. 1278-1288, 2008.

[189] G. Zheng, F. Patolsky, Y. Cui, W. U. Wang, and C. M. Lieber, "Multiplexed electrical detection of cancer markers with nanowire sensor arrays," Nat. Biotechnol., vol. 23, no. 10, pp. 1294-1301, 2005.

[190] L. Meseguer-Olmo, M. J. Ros-Nicolás, V. Vicente-Ortega, M. Alcaraz-Baños, M. ClavelSainz, D. Arcos, C. V. Ragel, M. Vallet-Regí, and C. Meseguer-Ortiz, “A bioactive sol-gel glass implant for in vivo gentamicin release. Experimental model in Rabbit," J. Orthop. Res., vol. 24, no. 3, pp. 454-460, 2006.

[191] H. Meng, M. Liong, T. Xia, Z. Li, Z. Ji, J. I. Zink, and A. E. Nel, "Engineered design of mesoporous silica nanoparticles to deliver doxorubicin and P-glycoprotein siRNA to overcome drug resistance in a cancer cell line," ACS Nano, vol. 4, no. 8, pp. 4539-4550, 2010. 
[192] J. M. Rosenholm, A. Meinander, E. Peuhu, R. Niemi, J. E. Eriksson, C. Sahlgren, and M. Lindén, "Targeting of porous hybrid silica nanoparticles to cancer cells," ACS Nano, vol. 3, no. 1, pp. 197-206, 2008.

[193] Q. Zhang, F. Liu, K. T. Nguyen, X. Ma, X. Wang, B. Xing, and Y. Zhao, "Multifunctional Mesoporous Silica Nanoparticles for Cancer-Targeted and Controlled Drug Delivery," Adv. Funct. Mater., vol. 22, no. 24, pp. 5144-5156, 2012.

[194] Z. G. Chen, P. W. Wang, B. Wei, X. M. Mo, and F. Z. Cui, "Electrospun collagenchitosan nanofiber: A biomimetic extracellular matrix for endothelial cell and smooth muscle cell," Acta Biomater., vol. 6, no. 2, pp. 372-382, 2010.

[195] M. D. Perry, B. C. Stuart, P. S. Banks, M. D. Feit, V. Yanovsky, and A. M. Rubenchik, "Ultrashort-pulse laser machining of dielectric materials," J. Appl. Phys., vol. 85, no. 9, pp. 6803-6810, 1999.

[196] A. Tavangar, B. Tan, and K. Venkatakrishnan, "Study of the formation of 3-D titania nanofibrous structure by MHz femtosecond laser in ambient air," J. Appl. Phys., vol. 113, no. 2, p. 023102, 2013.

[197] G. Odachi, R. Sakamoto, K. Hara, and T. Yagi, "Effect of air on debris formation in femtosecond laser ablation of crystalline Si,” Appl. Surf. Sci., vol. 282, pp. 525-530, 2013.

[198] M. Ullmann, S. K. Friedlander, and A. Schmidt-Ott, "Nanoparticle formation by laser ablation," J. Nanoparticle Res., vol. 4, no. 6, pp. 499-509, 2002.

[199] E. Biserni, A. Scarpellini, R. Brescia, D. Dellasega, A. Li Bassi, and P. Bruno, "Room temperature fabrication of silicon nanocrystals by pulsed laser deposition," 2014.

[200] P. P. Dey and A. Khare, "Fabrication of luminescent a-Si:SiO2 structures by direct irradiation of high power laser on silicon surface," Appl. Surf. Sci., vol. 307, pp. 77-85, 2014.

[201] R. Ravichandran, S. Gandhi, D. Sundaramurthi, S. Sethuraman, and U. M. Krishnan, "Hierarchical mesoporous silica nanofibers as multifunctional scaffolds for bone tissue regeneration," J. Biomater. Sci. Polym. Ed., vol. 24, no. 17, pp. 1988-2005, 2013.

[202] D. R. Nisbet, J. S. Forsythe, W. Shen, D. I. Finkelstein, and M. K. Horne, "Review paper: a review of the cellular response on electrospun nanofibers for tissue engineering," $J$. Biomater. Appl., 2008. 
[203] M. S. Lord, M. Foss, and F. Besenbacher, "Influence of nanoscale surface topography on protein adsorption and cellular response," Nano Today, vol. 5, no. 1, pp. 66-78, 2010.

[204] D. H. Jones, T. Nakashima, O. H. Sanchez, I. Kozieradzki, S. V. Komarova, I. Sarosi, S. Morony, E. Rubin, R. Sarao, C. V. Hojilla, and others, "Regulation of cancer cell migration and bone metastasis by RANKL," Nature, vol. 440, no. 7084, pp. 692-696, 2006.

[205] R. J. Dekker, J. D. de Bruijn, M. Stigter, F. Barrere, P. Layrolle, and C. A. van Blitterswijk, "Bone tissue engineering on amorphous carbonated apatite and crystalline octacalcium phosphate-coated titanium discs," Biomaterials, vol. 26, no. 25, pp. 52315239, 2005.

[206] R. A. Gittens, T. McLachlan, R. Olivares-Navarrete, Y. Cai, S. Berner, R. Tannenbaum, Z. Schwartz, K. H. Sandhage, and B. D. Boyan, "The effects of combined micron/submicron-scale surface roughness and nanoscale features on cell proliferation and differentiation," Biomaterials, vol. 32, no. 13, pp. 3395-3403, 2011.

[207] L. Zhao, S. Mei, P. K. Chu, Y. Zhang, and Z. Wu, "The influence of hierarchical hybrid micro/nano-textured titanium surface with titania nanotubes on osteoblast functions," Biomaterials, vol. 31, no. 19, pp. 5072-5082, 2010.

[208] C.-Y. Jin, B.-S. Zhu, X.-F. Wang, and Q.-H. Lu, "Cytotoxicity of titanium dioxide nanoparticles in mouse fibroblast cells," Chem. Res. Toxicol., vol. 21, no. 9, pp. 18711877, 2008.

[209] M. Webster, K. L. Witkin, and O. Cohen-Fix, "Sizing up the nucleus: nuclear shape, size and nuclear-envelope assembly," J. Cell Sci., vol. 122, no. 10, pp. 1477-1486, 2009.

[210] R. You, X. Li, Y. Liu, G. Liu, S. Lu, and M. Li, "Response of filopodia and lamellipodia to surface topography on micropatterned silk fibroin films," J. Biomed. Mater. Res. A, 2014.

[211] J. Lee, B. S. Kang, B. Hicks, T. F. Chancellor Jr, B. H. Chu, H.-T. Wang, B. G. Keselowsky, F. Ren, and T. P. Lele, "The control of cell adhesion and viability by zinc oxide nanorods," Biomaterials, vol. 29, no. 27, pp. 3743-3749, 2008.

[212] M. J. Sailor and J.-H. Park, "Hybrid nanoparticles for detection and treatment of cancer," Adv. Mater., vol. 24, no. 28, pp. 3779-3802, 2012. 
[213] B. Sahoo, K. S. P. Devi, S. Dutta, T. K. Maiti, P. Pramanik, and D. Dhara, "Biocompatible mesoporous silica-coated superparamagnetic manganese ferrite nanoparticles for targeted drug delivery and MR imaging applications," J. Colloid Interface Sci., vol. 431, pp. 3141, 2014.

[214] C. Li, T. Chen, I. Ocsoy, G. Zhu, E. Yasun, M. You, C. Wu, J. Zheng, E. Song, C. Z. Huang, and W. Tan, "Gold-Coated Fe3O4 nanoroses with five unique functions for cancer cell targeting, imaging, and therapy," Adv. Funct. Mater., vol. 24, no. 12, pp. 1772-1780, 2014.

[215] E. Yan, Y. Fan, Z. Sun, J. Gao, X. Hao, S. Pei, C. Wang, L. Sun, and D. Zhang, "Biocompatible core-shell electrospun nanofibers as potential application for chemotherapy against ovary cancer," Mater. Sci. Eng. C, vol. 41, pp. 217-223, 2014.

[216] A. Milgroom, M. Intrator, K. Madhavan, L. Mazzaro, R. Shandas, B. Liu, and D. Park, "Mesoporous silica nanoparticles as a breast-cancer targeting ultrasound contrast agent," Colloids Surf. B Biointerfaces, vol. 116, pp. 652-657, 2014.

[217] A. M. Kallinen, M. P. Sarparanta, D. Liu, E. M. Mäkilä, J. J. Salonen, J. T. Hirvonen, H. A. Santos, and A. J. Airaksinen, "In vivo evaluation of porous silicon and porous silicon solid lipid nanocomposites for passive targeting and imaging," Mol. Pharm., vol. 11, no. 8, pp. 2876-2886, 2014.

[218] F. Peng, Y. Su, X. Ji, Y. Zhong, X. Wei, and Y. He, "Doxorubicin-loaded silicon nanowires for the treatment of drug-resistant cancer cells," Biomaterials, vol. 35, no. 19, pp. 5188-5195, 2014.

[219] F. I. Thege, T. B. Lannin, T. N. Saha, S. Tsai, M. L. Kochman, M. A. Hollingsworth, A. D. Rhim, and B. J. Kirby, "Microfluidic immunocapture of circulating pancreatic cells using parallel EpCAM and MUC1 capture: Characterization, optimization and downstream analysis," Lab Chip - Miniaturisation Chem. Biol., vol. 14, no. 10, pp. 17751784, 2014.

[220] S. Dhanekar and S. Jain, "Porous silicon biosensor: Current status," Biosens. Bioelectron., vol. 41, no. 1, pp. 54-64, 2013.

[221] I. Fratoddi, I. Venditti, C. Cametti, and M. V. Russo, "Gold nanoparticles and gold nanoparticle-conjugates for delivery of therapeutic molecules. Progress and challenges," $J$. Mater. Chem. B, vol. 2, no. 27, pp. 4204-4220, 2014. 
[222] L. Dykman and N. Khlebtsov, "Gold nanoparticles in biomedical applications: recent advances and perspectives," Chem. Soc. Rev., vol. 41, no. 6, pp. 2256-2282, 2012.

[223] J. A. Webb and R. Bardhan, "Emerging advances in nanomedicine with engineered gold nanostructures," Nanoscale, vol. 6, no. 5, pp. 2502-2530, 2014.

[224] S. Shen, H. Tang, X. Zhang, J. Ren, Z. Pang, D. Wang, H. Gao, Y. Qian, X. Jiang, and W. Yang, "Targeting mesoporous silica-encapsulated gold nanorods for chemo-photothermal therapy with near-infrared radiation," Biomaterials, vol. 34, no. 12, pp. 3150-3158, 2013.

[225] X. Wang, H. Chen, Y. Zheng, M. Ma, Y. Chen, K. Zhang, D. Zeng, and J. Shi, “Aunanoparticle coated mesoporous silica nanocapsule-based multifunctional platform for ultrasound mediated imaging, cytoclasis and tumor ablation," Biomaterials, vol. 34, no. 8, pp. 2057-2068, 2013.

[226] E. G. Gamaly, A. V. Rode, and B. Luther-Davies, "Ultrafast ablation with high-pulse-rate lasers. Part I: Theoretical considerations," J. Appl. Phys., vol. 85, no. 8, pp. 4213-4221, 1999.

[227] J.-P. Sylvestre, A. V. Kabashin, E. Sacher, and M. Meunier, "Femtosecond laser ablation of gold in water: influence of the laser-produced plasma on the nanoparticle size distribution," Appl. Phys. A, vol. 80, no. 4, pp. 753-758, 2005.

[228] S. Mohapatra, Y. . Mishra, D. K. Avasthi, D. Kabiraj, J. Ghatak, and S. Varma, "Synthesis of gold-silicon core-shell nanoparticles with tunable localized surface plasmon resonance," Appl. Phys. Lett., vol. 92, no. 10, pp. 103105-1-103105-3, 2008.

[229] C. Wu, C. H. Crouch, L. Zhao, and E. Mazur, "Visible luminescence from silicon surfaces microstructured in air," Appl. Phys. Lett., vol. 81, no. 11, pp. 1999-2001, 2002.

[230] C. S. Chen, M. Mrksich, S. Huang, G. M. Whitesides, and D. E. Ingber, "Geometric control of cell life and death," Science, vol. 276, no. 5317, pp. 1425-1428, 1997.

[231] Y. H. Tan, S. E. Terrill, G. S. Paranjape, K. J. Stine, and M. R. Nichols, "The influence of gold surface texture on microglia morphology and activation," Biomater. Sci., vol. 2, no. 1, pp. 110-120, 2014.

[232] D. Pooja, S. Panyaram, H. Kulhari, S. S. Rachamalla, and R. Sistla, "Xanthan gum stabilized gold nanoparticles: Characterization, biocompatibility, stability and cytotoxicity," Carbohydr. Polym., vol. 110, pp. 1-9, 2014. 
[233] D. J. Lewis and Z. Pikramenou, "Lanthanide-coated gold nanoparticles for biomedical applications," Coord. Chem. Rev., vol. 273-274, pp. 213-225, 2014.

[234] M. J. Dalby, D. Giannaras, M. O. Riehle, N. Gadegaard, S. Affrossman, and A. S. G. Curtis, "Rapid fibroblast adhesion to $27 \mathrm{~nm}$ high polymer demixed nano-topography," Biomaterials, vol. 25, no. 1, pp. 77-83, 2004.

[235] J. M. Dang and K. W. Leong, "Myogenic induction of aligned mesenchymal stem cell sheets by culture on thermally responsive electrospun nanofibers," Adv. Mater., vol. 19, no. 19, pp. 2775-2779, 2007.

[236] J. Folkman and A. Moscona, "Role of cell shape in growth control," 1978.

[237] K. Anselme and M. Bigerelle, "Effect of a gold-palladium coating on the long-term adhesion of human osteoblasts on biocompatible metallic materials," Surf. Coat. Technol., vol. 200, no. 22-23 SPEC. ISS., pp. 6325-6330, 2006.

[238] P. M. Tiwari, K. Vig, V. A. Dennis, and S. R. Singh, "Functionalized gold nanoparticles and their biomedical applications," Nanomaterials, vol. 1, no. 1, pp. 31-63, 2011.

[239] R. Michel, J. W. Lussi, G. Csucs, I. Reviakine, G. Danuser, B. Ketterer, J. A. Hubbell, M. Textor, and N. D. Spencer, "Selective molecular assembly patterning: A new approach to micro-and nanochemical patterning of surfaces for biological applications," Langmuir, vol. 18, no. 8, pp. 3281-3287, 2002. 